\title{
U.S. Electric Utility Demand-Side Management 1996
}

\section{December 1997}

\author{
Energy Informatron Adminlstration \\ Office of Coal, Nuclear, Electric and Alternate Fuels \\ U.S. Department of Energy \\ Washington, DC 20585
}

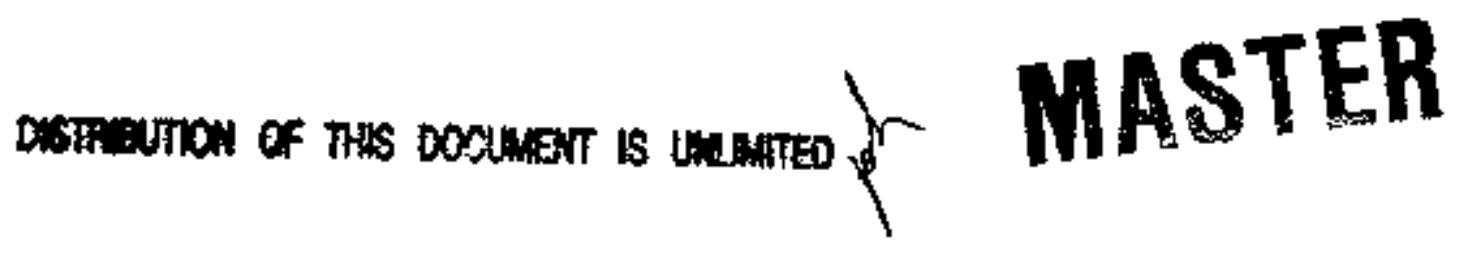

This report was prepared by the Energy information Administration. the indegendent statistical and anglytical agency within the U.S. Department of Energy. The information contained herein should be attributed to the Enexgy Information Administration and should not be construed as advocating or reflectitug any policy position of the Departenent of Energy or any other organization. 


\section{Preface}

The U.S. Electric Utility Demand-Sidt Management report is prepared by the Coal and Electric Data and Renewables Division; Office of Coal, Nuclear, Electric and Altertate Fuels; Energy Information Administration (EIA); U.S. Department of Energy. The report presents comprehensive information on electric power industry demand-side management (DSM) activities in the United States at the national, regional, and atility levels. The objective of the publication is to provide industry decision maikers, government policy makers, anajysts, and the general public with historical data that may be used in understanding DSM as it relates to the U.S. electric power industry. The first chapter, "Profile: U.S. Electric Uitility Demand-ijide Managemont," presents a general discussjon of DSM, its history, current issues, and a review of key statistics for the year. Subsequent chapters present discussions and more detailed data on energy savings, peak load reductions and costs attributable to DSM.

\section{Target Audlence}

In the private sector, the majority of users are researchers, analysts, and ultimately the policymaking and decisionmaking members of electric utility eonpanies. Financial and investment institutions, economic developnent organizations interested in new power plant construction, special interest groups. tobbyists, ejectric power associations, and the news medja are all prospective users of the iJ,S. Electric Utility Demand-Side Management report.

In the public sector, users incinde analysts, researchers, statisticians, and other profossionals engaged in reguhatory, poljcy, and prozrem activities fot Federat, State, and local governments. The Congress, other legislative bodies, State public service commissions, and other governsent groups share an jnterest in geatral trends and specific DShs data. This report can be used in analytic studjes to evaluate new or existing legistation.

\section{Source of Data}

Data published in the U.S. Electric Utility DemandSide Management report are compiled from the Form ElA-861, "Annual Electric Utility Report." The Form EIA-861 is a census of electric utilities in the United States, its territoties, and Puerto Rico. It is used to collect annua data on the production, sales, revenue from sales, and trate of electricity, as well as demand-side management from approximately 3,200 electric utilities in the United States. DSM data are reported on Schedule V, "Demand-Side Management information, " of Form EIA-861.

Questions regarding the contents of this document may be directed to:

Coal and Electric Data and Renewables Division

Energy Information Administration, BI-52

U.S. Departuent of Energy

1000 Ipdependence Avenue, S.W.

Washington, DC 20585-0650

Fax phone number (202) 426.1307

Questions of a general nstuse may be directed to:

Howard Walton (202/426-1156),

Internet E-Mail: hwalton eia.doe.gov

Director of the Coal and Electric Data and Renewables Division

Specific information on demand-side management may be directed to:

Linda M. Bromley (202/426-1164), Internet E-Mail: Ibromley 9 eia.đot.gov 


\section{Dreanumax}

Portions of this doenoment may be illegilale in dectronic longe prodines Imeges art produed from the beat arithible eriginil daciment 


\section{DISCLAIMIER}

This report was ptepared as an account of work sponsored by an apency of the Uoited States Government. Neither the United Slates Goverument not any agency therext, nor any of their templayeas, maked any warranty, exptest or implied, or

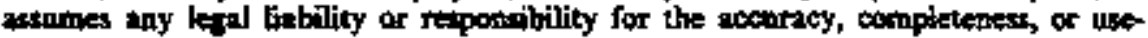
fulness of any information, apparatus, product, or process discloced, or represents

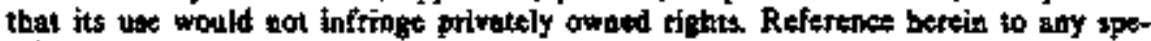
cific commerciol product, process, of service by totde name, trademart, manuiac-

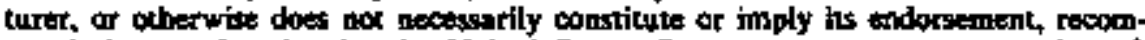
mendation, of favoring by the United States Gowernment or aby agency thereof. The views and opunions of authors expressed herein do not necessarily state or reilect those of the Uaited Stales Gowerninert or any agency therafi. 


\section{Contents}

Page

Profile: U.S. Electric Utility Demand-Side Managenent $\ldots \ldots \ldots \ldots \ldots \ldots \ldots \ldots \ldots \ldots \ldots \ldots \ldots \ldots \ldots$

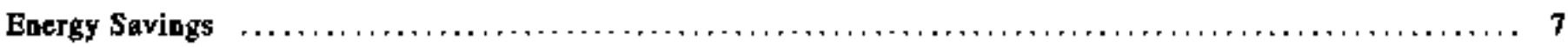

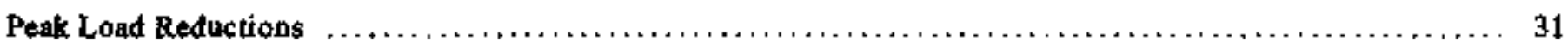

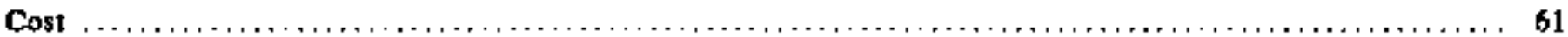

Appendices

A. Technical Notes $\ldots \ldots \ldots \ldots \ldots \ldots \ldots \ldots \ldots \ldots \ldots \ldots \ldots \ldots \ldots \ldots \ldots \ldots \ldots \ldots \ldots \ldots, \quad 87$

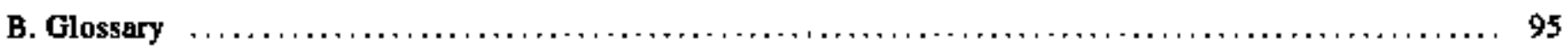




\section{Tables}

1. U.S. Electric Utility DSM Program Energy Savings, Actual and Potential Peak Load Reductions, and Cosi, 1992 Through 1996

2. U.S. Electric Utility DSM Program Energy Savings by Class of Ownership, 1992 Through 1996, 1997

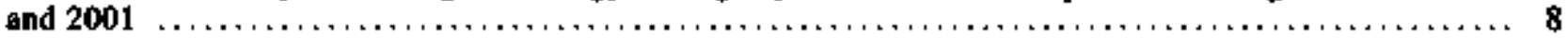

3. U.S. Electric Utitity DSM Program Energy Savings by Progtam Category, 1995, 1996, 1997, and 2001 10

4. Number of U.S. Eloctric Utilities with DSM Energy Efficiency Progtams by End Uses and Program

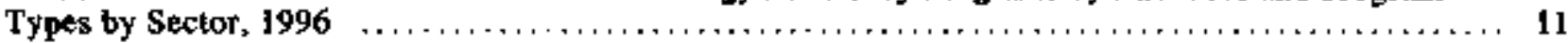

5. U.S. Electric Utility DSM Program Energy Savings by Sector, 1995 and $1996 \ldots \ldots \ldots \ldots \ldots \ldots \ldots, 11$

6. U.S. Electric Utility Incremental Energy Savings by Class of Ownership, 1995 and $1996 \ldots \ldots \ldots \ldots, 12$

7. U.S. Electric Utility Incremental Ebergy Savings by Progratn Category, 1995 and $1996 \ldots \ldots \ldots \ldots, 12$

8. U.S. Electric Utílity Incrementa! Energy Savings by Sector, 1995 and $1996 \ldots \ldots \ldots \ldots \ldots \ldots \ldots, 12$

9. U.S. Electric Utility Energy Savings by North American Electric Reliability Council Region and Hawaii by Class of Ownersbip, 1995, 1996, 1997, and $2001 \ldots \ldots \ldots \ldots \ldots \ldots \ldots \ldots \ldots \ldots \ldots$

10. U.S. Electric Utility Energy Savings by Nortb American Electric Reliability Council Region and Hawaii by DSM Program Category, 1996

11. U.S. Electric Utility Energy SaYings by Nortb American Electric Reliability Council Region asd

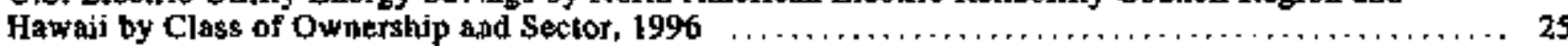

12. U.S. Blectric Utility Actual and Potential Peak Load Reductions by Class of Ownership, 1992 Through 1996,1997 , and 2001

13. U.S. Electric Utility Actual and jotontial Peak Load Reductions by DSM Program Category, 1995 .

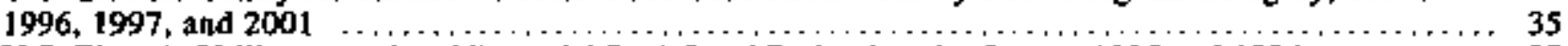

14. U.S. Electric Utility Actual and p'otential Peak Losd Reductions by Sector, 1995 and $1996 \ldots \ldots \ldots .37$

15. U.S. Electric Utjlity Incremental Actuat Poak Load Reductions by Class of Ownership, 1995 and 1996 37

16. U.S. Electric Utility Incremental Actual Peak Load Reductions by DSM Program Category, 1995 and

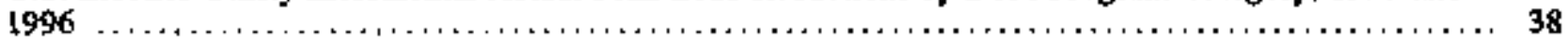

17. U.S. Electric Utility Incremental Actual Peak Load Reductions by Sectos, 1995 and $1996 \ldots \ldots \ldots$

18. U.S. Electric Utility Actual and Poiential Peak Load Reductlons by North American Electric Reliability Council Region and Hawaii by Utility, 1995, 1996, 1997, and $2001 \ldots \ldots \ldots \ldots \ldots \ldots \ldots \ldots \ldots$

19. U.S. Electric Utility Actual Peak Load Reductions by North American Electric Reliability Council

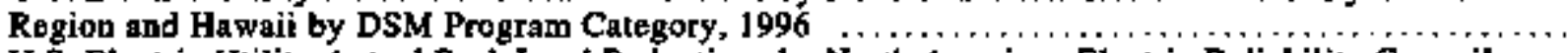

20. U.S. Electric Utility Actual Peak Load Reductions by North American Electric Reliability Council Region and Hawali by Class of Owhershjp and Sector, $1996 \ldots \ldots \ldots \ldots \ldots \ldots \ldots \ldots \ldots \ldots \ldots$

21. U.S. Electric Utility DSM Progran Costs by Class of Ownership, 1992 Through 1996, 1997, and 200163

22. U.S. Electric Utility DSM Program Costs by North American Electric Reliability Council Region and

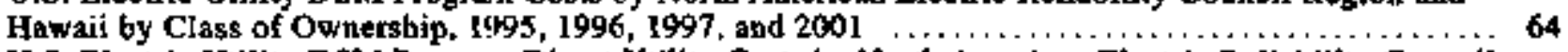

23. U.S. ESectric Uti]ity DSM Program Direct Utility Costs by North American Electric Reliability CounciS

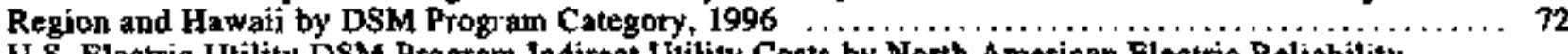

24. U.S. Electric Utility DSM Program Indirect Utility Costs by North American Electric Reliability

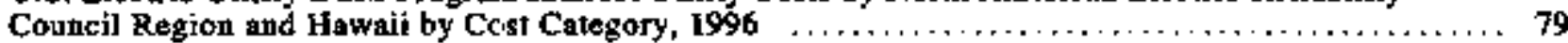

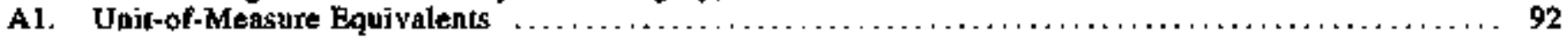




\section{Illustrations}

1. Number of U.S. Electric Utilities With and Without DSM Programs, 1996

2. U.S. Electric Utility DSM Program lncremental athd Annual Effects for Energy Saviogs and Actual and Potential Peak Load Reductions, 1996

3. Energy Savings as a Percentage of Retail Sales by U.S. Electric Utilities with DSM Energy Savings Programs and by Class of Ownership, 1996

4. The Top 25, 50, and 100 U.S. Electric Utilities with the Greatest DSM Program Energy Savings by Class of Ownership, 1996

5. Actual Peak Load Reductions as a Percentsge of Tolat Peak Losd by U.S. Electric Utitities with DSM Peak Load Reduction Programs and by Class of Owtership, 1996

6. The Top 25, 50, and 100 U.S. Electric Utilities with the Greatest DSM Program Peak Load Reductions

by Ctass of Ownership, $1996 \ldots \ldots \ldots \ldots \ldots \ldots \ldots \ldots \ldots \ldots \ldots \ldots \ldots \ldots \ldots \ldots \ldots \ldots \ldots \ldots$
J.S. Electric Utility Actual and Potentjal Peak Load Reductions by DSM Program Category, $1996 \ldots \ldots$

8. U.S. Electric Utility DSM Program Costs as a Percentage of Retail Revenue by Nomber of Utilities with DSM Costs, 1996

9. The Top 25, 50, and 100 U.S. Electric Utilities with the Greatest DSM Program Costs by Class of

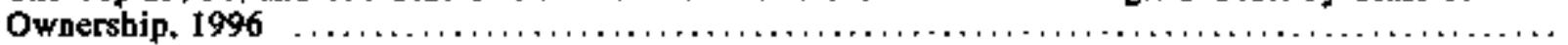

A1. Notth Atterican Etectric Reliability Council Regions for the Contiguous United States and Alaska ... 


\section{Profile: U.S. Electric Utility Demand-SIde Management}

This chapter provides a background of electric utility demand-side management (DSM) and pertineat statistics on DSM for large electric utilities' in the United States on various aspects of demand-side management.

\section{Background}

Demand-Side Management (DSM) consists of electric utilities' planning, implementing, and monitoring of activities designed to encourage consumers to modify their jevels and patterns of electricity consumption. These activilies are performed to benefit utilities, consumers, and society. Utilities implement DSM programs to achieve two basic objectives: energy efficiency and load managemeot. Euergy efficiency is primarily achieved through programs that reduce overall energy consumption of specific end-use devices and systems by promoting high-efficiency equiptrent and bullding design. Energy efficiency programs typically reduce energy consumption over many hours during the year. Load management programs, on the other hand, are designed to achieve load reductions: primarily implemented at the time of peak load. Load reduction programs have litule effect on total energy consumption. Electric utjlities have steadily increased DSM programs in the last decade to promole energy efficiency, and achieve cost effectivemess for both utilities and consamers, mainly by deferring the need to bujld new power plants. Energy efficiency programs also conserve tossil-fuel energy Bources and reduce air emissions.

The Energy Information Administration (ElA) collects data on DSM programs using six prograni categories:

Energy Efficiency programs are aimed st reducing the energy consuned by specific end-use devices and systems, without reducing the quality of energy services provided. These programs roduce overall electricity consumption over many hours during the year, although the greatest impacts of cost-effective programs often coincide with periods of peak usage. Such savings are generaliy achieved by substituting technologically more advanced equipment to produce equal levels of energy services (e.g, lighting, heatiog, motor drive) with less electricity. Examples include energy saving appljances and lighting, high-efficiency beating, ventilating and air conditioning (HVAC) systems of control modification, effjeient building design, advanced electric motors and drive systems, and heat recovery systems. Energy efficiency programs frequently incorporate tinancing or firancial incentives for participation.

Direet Load Contral represents the consumer load that can be inlecrupted during perjods of peak demand by the utility system operator directly interrupting power supply to individual appliances or equipment. Direct Load Control usually involves residential consumers who, for example, allow the utility to periodically interrupt service to air conditioning units duríng the hours of peak joat.

Interruptible Load accounts for the consumer load that, in accordance with contractual arrangements, cas be interrupted during periods of peak load, either by direct control of the utility system operator or by action of the consumer, at the direct request of the system operatos. For example, large commercial and industrial consumers may obtain discount interruptible rates for agreeing to reduce electrical loads upon request from the utility, usually as a strategy to reduce peak load.

Other Load Managenent refers to programs other than direce load control and interruptible load that limit peak loads, shift peak load from on-peak to offpeak hours, or encourage consumers to respond to changes in the utility's cost of providing power. ${ }^{2}$ Included are tecbnologies that primarily shift all or part of a load from one time of day to another and also may affeet overall energy consumption. Examples inclute space heating and water hexting storage systems. cool storage systems, and Joad limitiag devices in energy management systems. This category also includes programs that aggressively promote lime-of-use (TOU) tates and other intovative rates such as real-tine pricing. These rates are intended to reduce consumer bills and shift hours of operation of equipment from on-peak to off-peak or high-cost to low-cest periods through the application of timedifferentiated rates.

Other Demand-Side Management are those prograns that capture effects of DSM programs that cannot be meaningfully included in any of the other

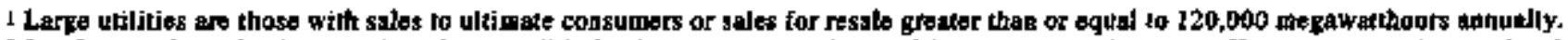

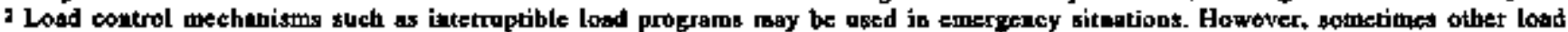

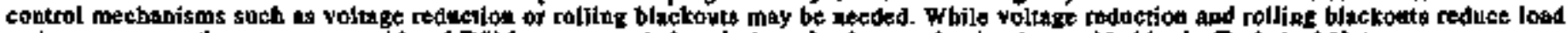

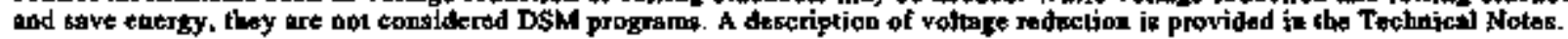


progratn categories. lncluded are programs that promote consumers' substitution of olher types of onergy for electricity and self-generation of electricity for the consumers' awn use.

Load Bullding programs are aimed at increasing the use of existing electric equipment or the additjon of electric equipment. Examples include industrial technologies such as induction heating and mielting, direct arc furnaces, and infrared drying; cooking for commercial esiablishments; and heal purips for residences. Load Building includes ptograms that promote the substitution of electricity for other forms of energy, Load Building promotes load growth and is not included in this publication.

The concept of energy efficiency began in the 1970's in response to increasing capital costi, jncreasing electricity demand, rising electricity prices, and increased public awareness of energy resourees and conservation. Federal regulalors and State public service commissions responded with ut.lity policies that contributed to the evolution of DSM. Federal legislation includes the Energy Policy and Conservation Act (1975), Energy Conservation and Production Act (1976), and the National Energy Conservation Policy Act (1978). These three Acts provided the technical basis for utility conservation and load onanagement programs. The Public Utility Regulatory Policies Act (1978) required State public service conmissions to consider tate-making standards that further the purposes of end-use conservation, utility efficiency, and equitable rates. It also requited State public servjce comnuissions to revjew cost allocations across concumer classes, the accurscy of declining block rates in toflecting actual casts, time-of-day and seasonal tates, interruptible rates, and load manggement techniques. The Paciftc Northwest Electric Power Planníng and Conservation Act (1980) and Hoover Power Plant Act (1984) encoturaged DSM through the Federal power marketing atministrations.

The National Appliance Energy Conservation Act (1987), Clean Ajr Act and its Argendments (1990), and the Energy Policy Act (1992) are the most recent Federal legistation affecting DSM. The Clean Air Act Amendments of 1990 internalized the cost of environmental externalities, specifically sulfur diuxide emissions, through the adoption of a market-bused system of emission control in which utilities are jssued allowances, each allowing the emission of one ton of sulfur dioxide per year. This system encourages utilities to reduce emissions in the most cost effective manner and sell or trade excess akjowances.

The Energy Policy Act of 1992 (BPACT) represents the continuing Federal Interest in encourating energy efficiency. EPACT requires State public service commissions to consider standards that will require utilities to emplay Integsated Resousce Planring (IRP). Consequently, most significant regulatoty requisements effecting DSM data are occurring at the State level. IRP differs from conventional resource planning in that utilities consider both demand- and supply-side resources as options for meeting future electricity requirements, rather than just supply-side resources. Specifically, a utility is able to assume a decrease in demand as a result of DSM programs when planning to meet future electricity needs, rather than increasing generation.

One key element in the DSM program planning and selection process is the identification and evaluation of consumer characteristics that influence acceptance and responses to DSM prograns. Among consumer characteristics that isfluence the success of a program are demographics, incone, knowlodge, awareness, attitude, and motivation. External influences such as economic conditions, epergy prices, technologies, regulation, and tax credits also influence consurners' decisions regarding fuel, appliance choices, and equipment efficiency. Another key element is the identification of utility considerations that affect resource requirements and the cost of alternative resource options. In a regulated industry, utility considerations are focused on the inleraction of load shape distribution effects and regulatory compliance.

To promote DSM. State regulatory commissions developed financial incentives, such as 1) authorizing utitities to seek tecovery of DSM program costs and lost revenues, and 2) granting utilities higher rates of return. These incentives are meant to neutralize the lost sales and revenues attributable to DSM. To compare DSM programs with other demand- and supply-side resources, regulators have developed standardized benefit/cost tests. Four prinary tests are widely used to identify cosl-effective DSM programs. For each test, the net present value and tenefit/cost ratio can be determinte. The present value equals total benefits of the program less total cost; the benefit/cost ratio is the ratio of total benefits to total costs. Based on these values, the atility calt prioritize DSM programs to determine whuch, if any, might be iniplemented.

The Utility Cost Test measures the net change in a utility's revenue requirement resulting from a DSM program. The test compares the reduction in marginal energy and demand costs with utility program costs, incentive payments, and increased supply costs for period in which load is increased. Designed to focus on a utility's revenue requirement, the test doos not include any net costs incurted by participants.

The Partieipant Cost Test measures the benefits and costs of a DSM program to a customet by comparing the reduction in the customer's ntility bill, plus any incentive paid by the atility, with the customer's outof-pocket expenses. The test is often used as "first-cut" in ranking program desirabjlity and gauging potential program participation rates.

The Total Resource Cost Test measures the net costs of a DSM program as a resource option based on the total cosis of the program, including both participant and utlity costs. Like the utility cost test, it measures benefits as reductions to energy and denand costs, but also includes a review of all program costs, including installation, operation, maintenance, and administration, no matter who pays for them.

The Rate Inpact Measnre Test measures the direction and magnitude of the expected changes in rates 
for atl enstomers when a atility implements a DSM program. The equation functions initially in the same manner as the utility cost test, comparing avoided supply cost savings with cost to the utility. It also measures the revenue-sbifting effect unique to DSM when costs must be spread over a smaller sales volvme. The shift reduces revente requirements, but not to the same extent as sales are reduced by DSM programs. The difference causes an increase in rates on a cents per kilowatthour basis. If a utility has excess capacity and its average costs exceed its marginal costs, a DSM program wi]l ljkely increase rates. The converse is true when marginal costs are forecast to exceed average costs.

\section{Current losues and Trends}

Throughout the United States, States are taking action to transform the electric power industry from a regulated monopoly into a competitive business. Most States are actively considering proposals for restrticturing the electric power industry, inclading options for deregulating the generation segment of the industry and providing retail access. Fourteen States including California, New York, and Arizona have enacted statulos and/or adopted policies that will create a conpetitjve retail access market. Eleven States including Massachusetts, Wasbington, and Michigan have pilot projects to test limited relail competition. Such changes are affecting utility DSM activities and could sjenificantly change the financing, structure, and delivery of end use energy services.

Traditionally, utility DSM prograris have beea deve]oped through an integrated resource planning process which compared the cost of DSM programs to the cost of other resources and are approved by State Public Utility Comnissions. In a competitivo market, regulated utiljties may not retain their obligation to provide generation services and regulatory oversight of their DSM programs. Additionally. competition is creating pressure for utilities 10 cut costs. In some instances, this bas resulked in a reduction in planned DSM expenditures and a shift away from customer rebale programs. Further, to the extent utility generation revenues ultimately may be based on competitive market prices, a conflict could emerge between the interests of generation owning utilities in bigher generation prices and the effects of some DSM programs to reduce demand and possibly to thelp hold down conpetitive prices for generation. These factors could eontribute to slower growth in energy sovings from DSM programs.
Now retailing activities are omerging as competition grows in the electric power industry. These jnclude increased btiljty atlention to marketing and the activjties of new brokers and energy service companies. These new energy retailers can be expected to offer customers packages of services that inelede olectricity (and in some cases natural gas), finapcial strvices to hedge price uncertainty, and expanded energy management services designed to allow consumers to adjust their energy usage to changing electricity prices. Demand-side services witl be compotitively marketed as a means of helping consumers manage their energy bills. These services may include automated energy management linked to a comtiunications system that provides consumers and their energy managemest systems access to changing bourly electricity prices.

Regulators and legishators in some States ate likely to set aside funds collected from all consumers connected to the distribution system to support energy efficiency programs. The California restructuring legjslation bas used this approach to require usilities to purchase energy efficiency savings under standard offers.

Utilities in the Pacific Northwest and New England have formet consortiums to support energy efficiency market transformation, programs that attempt to create lasting changes in markets for energy efficient products. Such efforts may represent a more econotnical way to achieve long-term energy savings.

Even though incremental savings from energy effciency programs in 1996 were less than the savings achioved in 1995 overall energy savings increased. This suggests that efficiency programs are continuing to play a significant role in the Nation's resource mix, even as it changes to reflect the development of a more competitive electric power industry.

In 1996, 1003 of the 3,199 electric utilities in the United States reported having DSM prograns. Of these 1003 electric utilities, 573 are classified as large and 430 as smalt. ${ }^{3}$ The 1003 utilities accounted for approximately 7 percent of the lotal retail sales of electricity in the United States.

In 1996, energy savings for the $\mathbf{5 7 3}$ large utilitios was 61,842 million kitowatthours ( $(\mathrm{Wb})$ an increase of 4,421 million $k$ Wh over the 57,421 million $k W h$ reported in 1995. These entergy savings represent 2.0 percent of annual electric sales to ultimate consumers in 1996 of $3,097,810$ million $\mathrm{kWh} .4$

\footnotetext{
${ }^{3}$ Upless otherwise s1ated, the discussions and statistics that are contained in this publication are for Jarte otiltizes only. Large utilities are

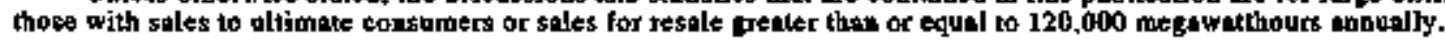

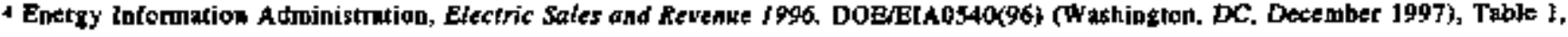
p. 5 .
} 
Actual peak load reductions for large utjlities in 1996 are $29,893 \mathrm{MW}$, an increase of 1.1 percent, from 29,56t megawatts (MW) in 1995. These actual peak load redactions are approximalely 4 percent of the cotal peak load in the United States. Potential peak load reductions in 1996 was $48,344 \mathrm{MW}$, an increase of 2.8 percent, from $47,029 \mathrm{MW}$ in 1995. DSM costs were approximbately $\$ 1.9$ bilition in 1996, a decrease of 21.5 percent.

Incremental effects are those caused by aow programs and new participants in existing programs for the current reporting year. For 1996, iocremental energy savings for large utilities were 6,844 mijlion $\mathbf{k W h}$ and incremental actual peak load reductions were 3,689 MW (Figure 2)."

Tahle 1. U.S. Flectric Utifity DSM Program Enerty Sovings, Actual and Potential Peak Land Reductions, and Cost, 1992 Throudi 1996

\begin{tabular}{|c|c|c|c|c|c|}
\hline tited & $15 \boldsymbol{z}$ & $\operatorname{tos}$ & 194 & 19\% & 15: \\
\hline 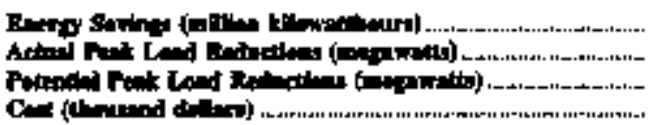 & $\begin{array}{r}35,364 \\
17,204 \\
32,442 \\
2,314,094\end{array}$ & 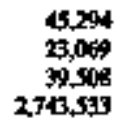 & $\begin{array}{r}52,489 \\
25,001 \\
42,917 \\
2,715,657\end{array}$ & $\begin{array}{r}57,421 \\
29,561 \\
7,0,09 \\
2,421,261\end{array}$ & 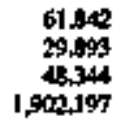 \\
\hline
\end{tabular}

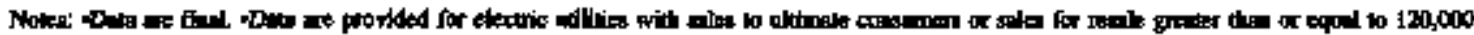

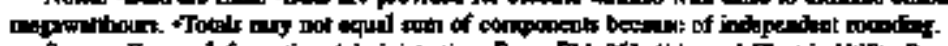

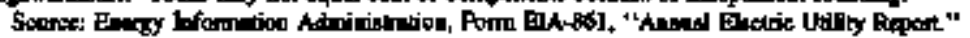

5 It is incorrect to assume thal 1995 ann discrepancy inclade incremestal effects being annuglized, atd the effocts of participants drapping aut of programs that are not included in incremental effects. 
Flgure 1. Number of U.S. Electric Utilites with and without DSH Programs, 1996

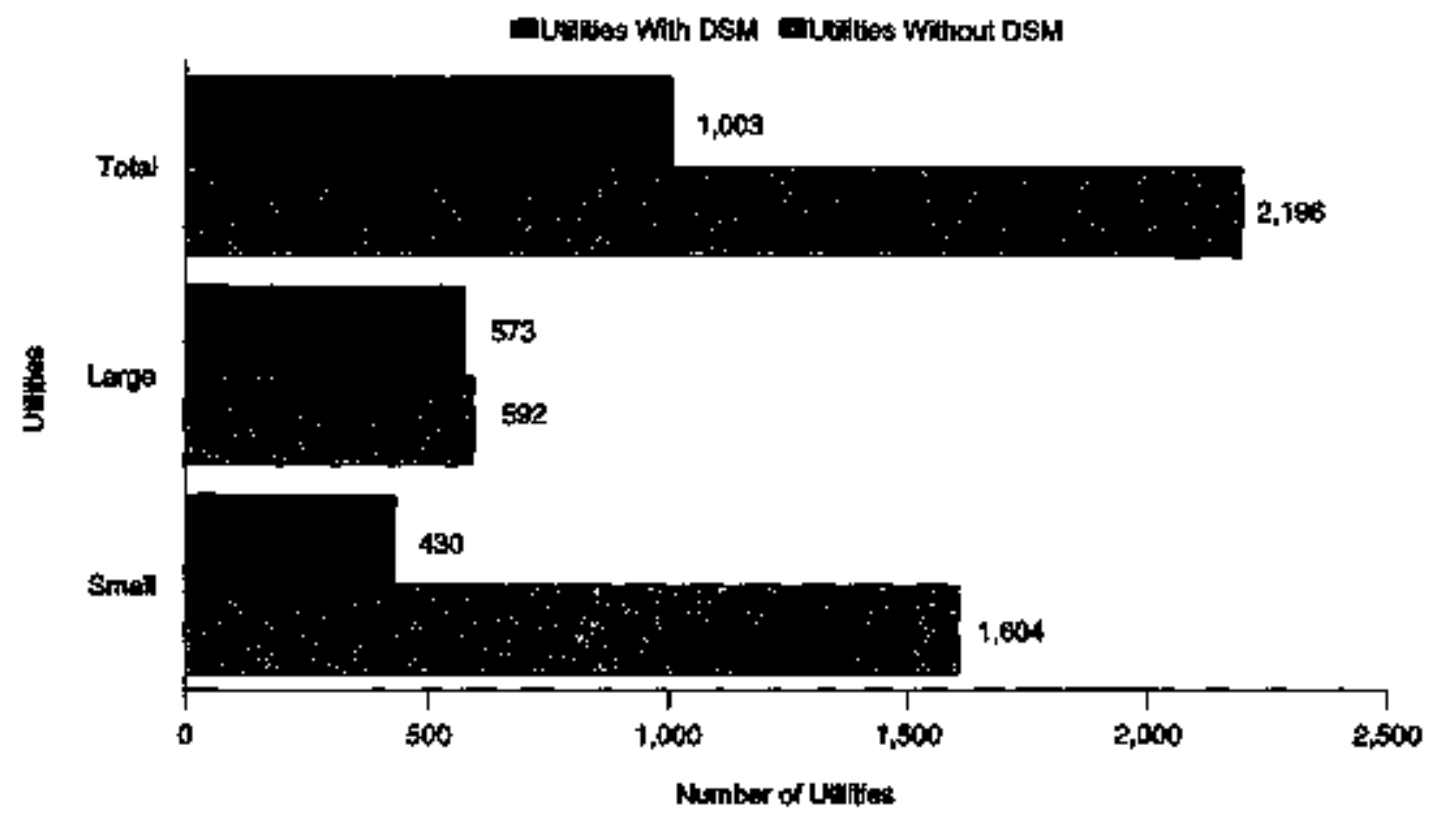

Source: Energy Informalton Adnunistration, Form ElA-961, "Anmual Electric Utlity Report."

Figure 2. U.S. Electric Lflity DSU Program Incrementol and Annuad Ettecto for Energy Savinga and Actual and Potentled Peak Load Reductions, 1996

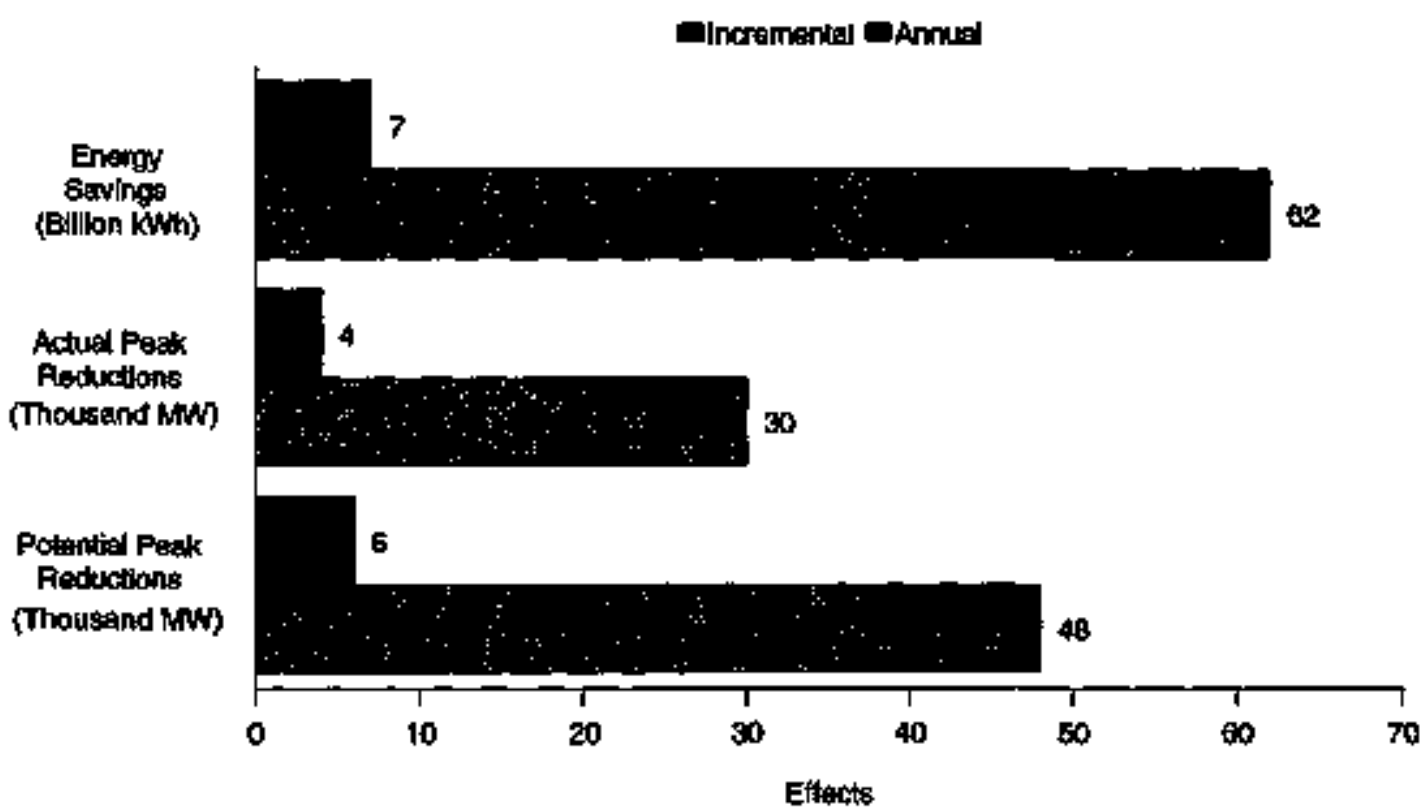

Source: Enerdy Iniormation Administralion, Form EIA-861, "Annusa Electric Lttiny Report" 



\section{Energy Savings}

Energy savings represent a decrease in the amount of electricity (measured in kilowathours $(\mathrm{kWh})$ ) that wonld have otherwise been consumed, absent of DSM. Energy savings primarily result from energy efficiency programs, but also result from load management and other DSM programs. Examples of energy efficiency programs include the promotion of energy soving appliances and lighting; bigh-efficiency heating and air conditioning systems (HVAC) and control modiffeation; enetgy efficient building designs; advanced electric motors and drive systems; and heat recovery systems.

The future of electric utility sponsored energy efficiency programs is uncertain due to competition in the alectric utility industry. In a competjtive environment, a utility would have little incentive to reduce energy sales (one of the objectives of energy efficiency programs).

In 1996, energy saviags increased 7.7 percent to 61,842 million $\mathrm{kWh}$ from the 1995 level of 57,421 million kWh. Fot 1997, energy savipgs are forecasted to increase 3.9 percent to 64,252 million $\mathrm{kWh}$, and for 2001, energy savings are forecasted to increase at an anuual rate of 3.9 percent to 74,552 nillion $\mathrm{kWh}$ (Table 2). The decline in the rate of increase, compared with prior years, ts due to many factors. For example, electric utilities are cautious about energy efficiency programs because of competition in the electric power industry, and saturation of the energy effjeiency market.

In 1996, energy savings represented a reduction in electricity sales by electric utilities of 2.0 percent. ${ }^{6}$ Approximately 45.6 percent of utilities that had energy saving programs reduced their energy sales by more than 1 percent in 1996 (Figute 3). Investorowned utilities represented the greatest energy savings as a percentage of sales in 1996.

The 100 utilities with the greatest energy savings accounted for 95.5 percent of total energy savings. The 50 and 25 utilities with the greatest energy savings accounted for 86.3 percent and 71.2 percent of total energy savings (Figure 4). These 100, 50, and 25 utilities with the greatest energy savings represented 55.4 percent, 36.6 percent, and 25.9 percent, respectively, of total retail sales of electricity in the United States for 1996.
Investor-owned utilities accerted for 81.5 percent of energy savings in 1996; publicly owned utilities accounted for 7.3 percent; cooperatives, .8 porcent; and Federalty owned utilities, 10.4 percent." From 1995 to 1996, investor owned electric utilities increased energy savings by 4.8 percent. Savings by pablicly owned utilities increased 39.4 percent. Savings by cooperatives and Federal electric utilities increased 127.4 percent and 9.2 percent. The largest increase over 1995 was for investor-owned electric utilities, increasing 2,322 million $\mathrm{kWh}$. However, from 1996 to 1997 , the forecasted rate of increase for investor-owaed electric utilities foll to 2.9 percent, while it increased to 15.3 percent for cooperatives. From 1996 to 1997, publicly owned utilities and Federal electric ntilities energy savings are predicted to increase 10.4 and 6.0 percent, respectively. Froton 1997 to 2001, projected energy savings are expected to increase in all clisses of owmership, with the largest percent increases, 5.9 and 4.4 percent annually, for publicly owned electric utilities and cooperalives, respectfully. The largest increase overall is predicted for investor-owned utilities.

In 1996, energy efficiency programs accounted for 96.8 percent of the energy savings. The primary objective of most other DSM programs is peak load reductions. Direct load control, internuptible lond, other load management, and other DSM programs together accounted for the remaining 3.2 percent of energy sovings. Energy savings from energy efficiency progtams increased 8.2 percent over the 1995 level. Enetgy savings decteased in all other categories, except direct load control and "other" DSM programs. For 1997, energy efficiency programs are predicted to continue to account for the greatest share of energy savings, 98.0 percent. The greatest percentage of increase is predicted for interruptible load control, which is expected to increase by $\mathbf{3 5 . 4}$ percent by 1997. By 2001, energy efficlency programs are expected to increase energy savings by an additional 10,021 million $\mathrm{kWh}$ over projected 1997 levels (Table 3).

During the year, more uttlities repotted having onergy efficiency prograns in place in the residential sector than in the commercial or industrial sectors. However, the commetcial and industrial seetors still contributed a large percentage of energy savings due to economles of scale (i.e., a commercial building participating in an efficient lighting program will have greater entergy savings than a single residential building). Bnergy

6 Total U.S. electric utility swles to ujtimate costumers for 1996 were 3,097,810 million kWh (Electric Sales ond Reverke 1990).

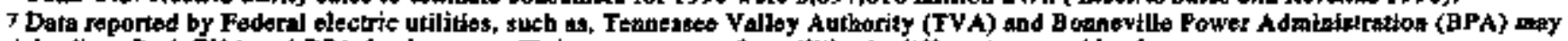

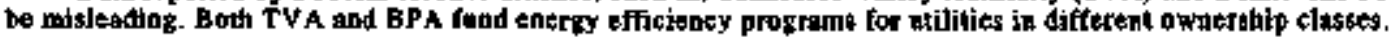


efficiency end-use programs in the resiljential sector were primarily for heating systems, cooling systems, and water heating. More utilities had fighting and cooling systems programs for the commercial sector, while the industrial sector focused on lighting and advanced motor programs. Across all sectors, more utitities used energy audits than other programs, folJowed by rebates (Table 4).

The coimutercial sector accounted for $\mathbf{4 7 . 2}$ percent of energy savings in 1996, followed by the residential, industrial, and other sextors with 33.3 percent, 17.0 percent, and 2.6 percent, respectively. Among the major consumer sectors, the greatest percentage of increase from 1995 to 1996 was in the other sector, with 16.0 percent more energy stavings (Table 5).

In 1996, incremental energy savings (the savings achieved by new programs and new participants in existing programs in a given year) decreased from $\$, 222$ million $k W h$ in 1995 to 6,844 million $k W h$ for large atilities but decreased from 20 million kWh to 13 million $\mathrm{kWh}$ for small utilities. By class of owbefship, targe investor-owned utilities accounted for 81.7 percent of incremental entricy savibjss. Publicly owned electric utilities and cooperatives both showed an increase in incremental energy savings is 1996 (Table 6).

By program category, incrementai energy savings for large utilities in 1996 decreased in energy efficiency and other load management. For small electric utilities in 1996, energy officiency programs decteased 9 million kWh (Table 7).

The commercial sector accounted for 31.6 percent of incremental energy savings, 3,540 million $\mathrm{kWh}$; the residential sector accounted for 17.3 percent. 1.186 millton $\mathrm{kWh}$; and the industrial sector accounted for 26.1 pereenh, 1.789 million $\mathrm{kWh}$.

The NERC region with the preatest percentage of energy sayings was Western Systemls Coordinating Council (WSCC), accounting for 38.3 percent of energy savings in 1996. The wSCC had the most energy savings because Bonneville Power Admiaistration and Southern California Bdison Company had the two largest energy efficiency programs of all electric utilities. The region with the second largest energy savings was Southeastern Electric Reliability Council (SERC), with 16.8 percent of total energy savings. In 1996, these two regions combined accousted for 55.1 percent of total U.S. onergy savings.

For 1997, the greatest percentage of incroase, 17.5 percent, in energy savings is predicted for the Mid. Atlantic Area Council (MAAC) region. The MAAC region is also expected to have the greatest annulual rate of growth in energy savings from 1997 to 2001 at 9.9 percent (Table 9).

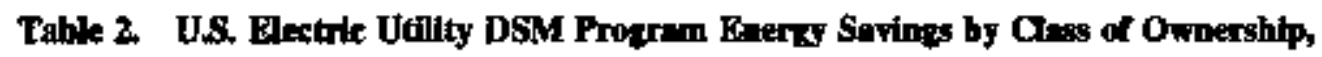
1992 Through 1996, 1997 and 2001 (Million Kilowathours)

\begin{tabular}{|c|c|c|c|c|c|c|c|}
\hline \multirow{2}{*}{ 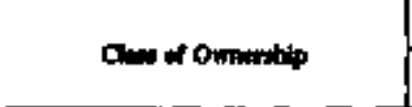 } & \multicolumn{5}{|c|}{ 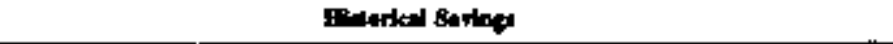 } & \multicolumn{2}{|c|}{ Probeded Btricp } \\
\hline & rys & 15 & 154 & yes & 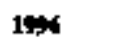 & 199 & 2001 \\
\hline 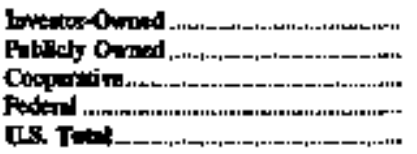 & $\begin{array}{r}25,926 \\
2,416 \\
400 \\
6,92 \\
3,913\end{array}$ & $\begin{array}{r}35.677 \\
2.562 \\
705 \\
6.950 \\
4.794\end{array}$ & $\begin{array}{r}4,132 \\
2,956 \\
560 \\
7,806 \\
7,403\end{array}$ & $\begin{array}{r}4,060 \\
3,118 \\
2,01 \\
5,911 \\
5,421\end{array}$ & $\begin{array}{r}5,342 \\
4,486 \\
343 \\
6,482 \\
01242\end{array}$ & $\begin{array}{r}51,960 \\
4,950 \\
600 \\
6,936 \\
4,450\end{array}$ & $\begin{array}{r}60,100 \\
6,202 \\
717 \\
7,511 \\
\text { Tititit }\end{array}$ \\
\hline
\end{tabular}

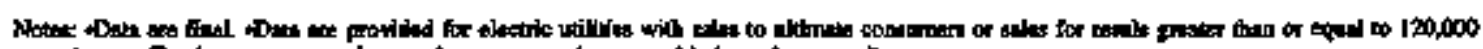

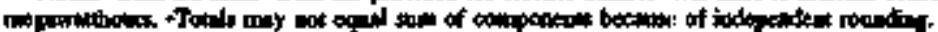

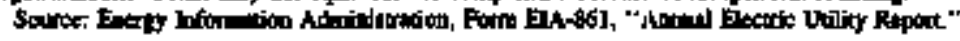


Figure 3. Energy Sevingt at a Percentuge of Rottil Salos by U.S. Eloctric Utilties with DSN Energy Sevinge Programs and Salos to Ulitmote Consumers by Class of Ownerahip, 1906

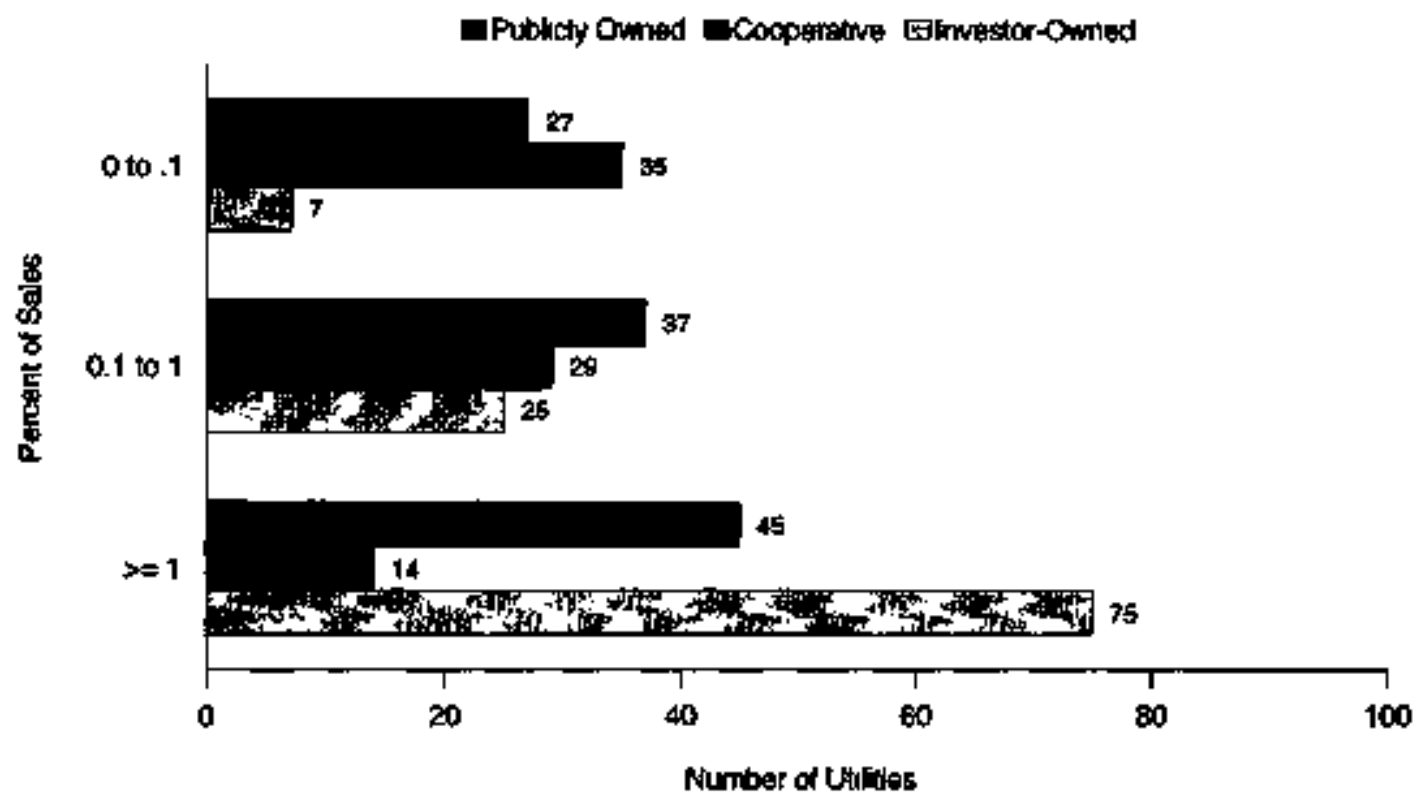

Nole: Graph inctudes only large utitities that reported energy savings.

Source: Energy Intormation Administration, Form EIA*\$61، "Annual Electric Utitity Repon."

Flgure 4. The Top 25, 50, and 100 U.S. Electric Unition with the Qreatest DSM Program Energy Sevinge by Class of Owmershlp, 1996

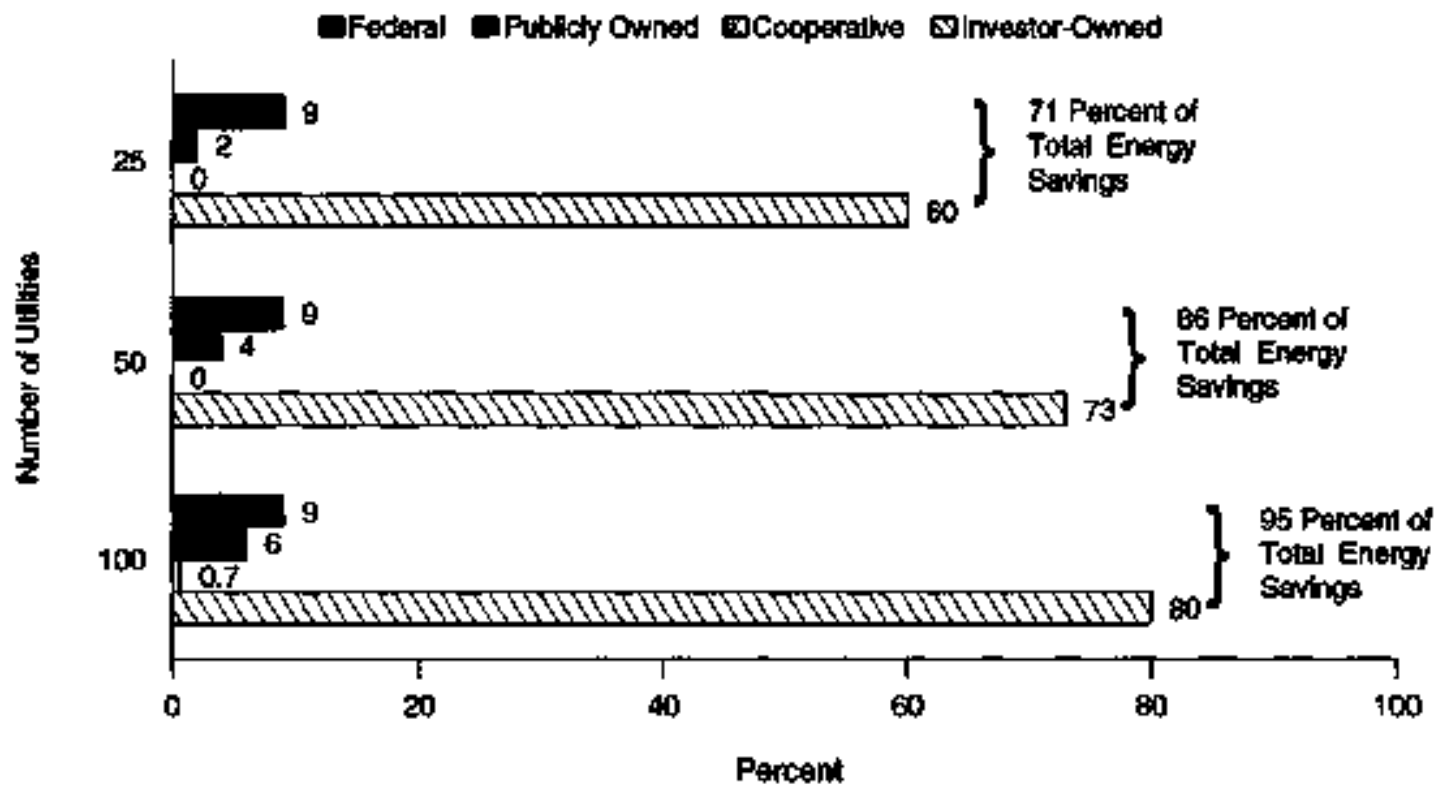

Note: Graph includes only large utilities that reported energy savings. No cooperatives were included in the top 25 or 50 vithlies.

Source: Energy Intormation Admunistratlon, Form ElA-681, "Anmual Electric Utility Report." 
Table 3. U.S. Dlectric Utility DSM Propram Enerw Sevings by Progann Category, 1995, 1996, 1997, and 2001

(Million Kilowathowr)

\begin{tabular}{|c|c|c|}
\hline \multirow{2}{*}{ Pro,pre Caterory } & \multicolumn{2}{|c|}{ 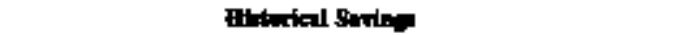 } \\
\hline & tos & 1906 \\
\hline \multirow[t]{3}{*}{ 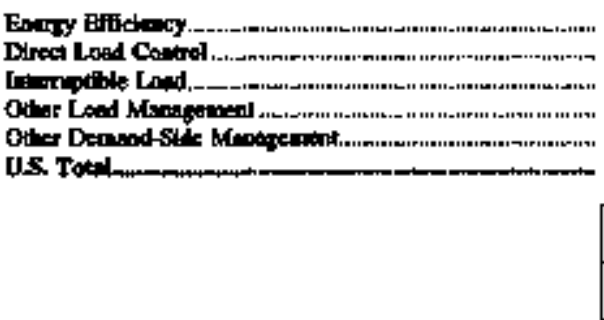 } & $\begin{array}{r}35,328 \\
133 \\
434 \\
297 \\
1,239 \\
77+17\end{array}$ & $\begin{array}{r}59,853 \\
134 \\
362 \\
-196 \\
1,609 \\
61,49\end{array}$ \\
\hline & \multicolumn{2}{|c|}{ Prejected sentar } \\
\hline & $1 ; 0 \pi$ & tont \\
\hline 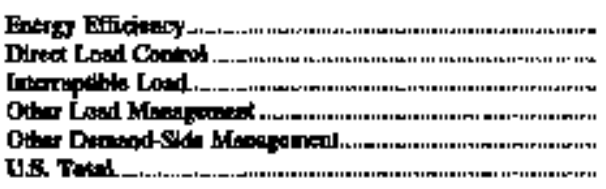 & $\begin{array}{r}6,949 \\
139 \\
490 \\
-303 \\
957 \\
4,248\end{array}$ & $\begin{array}{r}2,900 \\
161 \\
208 \\
-337 \\
1,029 \\
34,001\end{array}$ \\
\hline
\end{tabular}

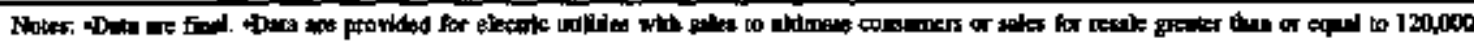

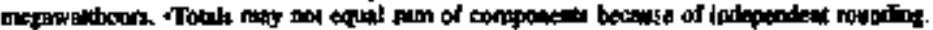

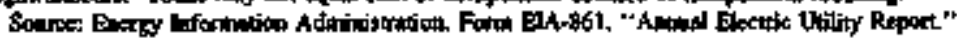


Table 4. Number of U.S. Electrk Utiltes with DSM Eneray Buiciency Prograngs by End Usea and Program Types by Sector, $19 \%$

\begin{tabular}{|c|c|c|c|}
\hline \multirow{2}{*}{ TRM } & \multicolumn{3}{|c|}{ Sectitr } \\
\hline & 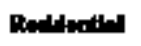 & 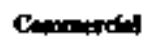 & Indinitrial \\
\hline 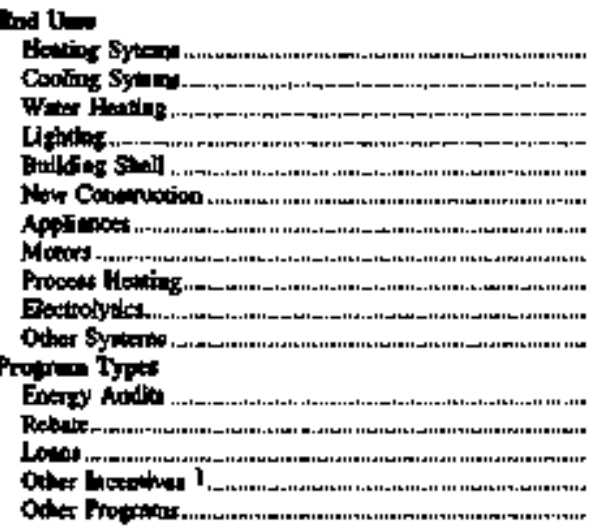 & $\begin{array}{r}278 \\
274 \\
292 \\
191 \\
192 \\
207 \\
130 \\
- \\
= \\
15 \\
303 \\
256 \\
138 \\
03 \\
50\end{array}$ & $\begin{array}{r}191 \\
267 \\
198 \\
214 \\
126 \\
132 \\
65 \\
143 \\
47 \\
9 \\
27 \\
263 \\
196 \\
91 \\
69 \\
47\end{array}$ & $\begin{array}{r}207 \\
130 \\
101 \\
151 \\
86 \\
93 \\
42 \\
164 \\
80 \\
22 \\
27 \\
198 \\
133 \\
62 \\
63 \\
45\end{array}$ \\
\hline
\end{tabular}

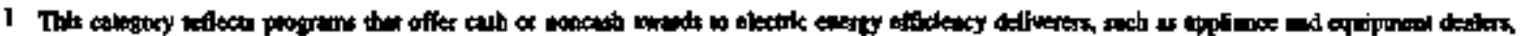

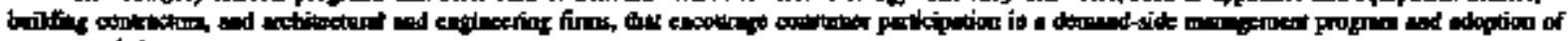
necompeoded moted.

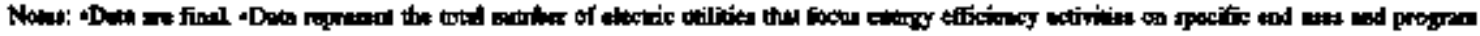
STp

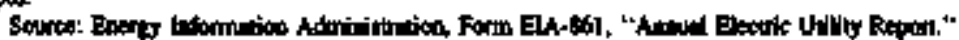

Tahlie 5. U.S. Electric Uthity DSM Program Enera Sarings by Sector, 19s5 and 1996 (Million Kilowethours)

\begin{tabular}{|c|c|c|}
\hline Secter & 1sos & 1506 \\
\hline 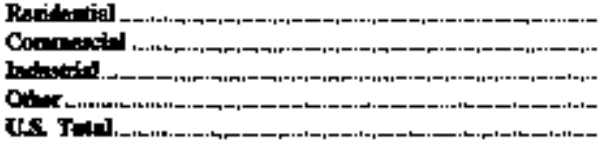 & $\begin{array}{r}2,1,253 \\
26,187 \\
9,620 \\
1,360 \\
57,421\end{array}$ & $\begin{array}{r}20,585 \\
29,1 \times 5 \\
10,93 \\
1,5,8 \\
1+\$ 2\end{array}$ \\
\hline
\end{tabular}

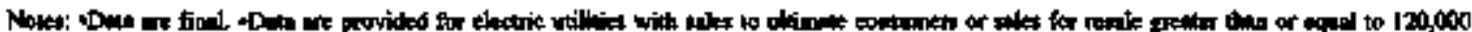

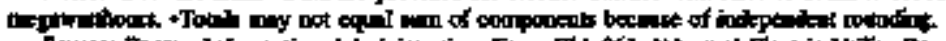

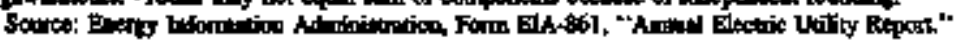


Table 6. U.S. Electric Utility Incremenal Energy Savings by Class of Owaership, 1995 and 1996 (Milion Ki]owatthours)

\begin{tabular}{|c|c|c|c|c|c|c|}
\hline \multirow{2}{*}{ 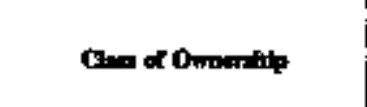 } & \multicolumn{2}{|c|}{ Late Ut ital 1} & \multicolumn{2}{|c|}{ small dittias 2} & \multicolumn{2}{|c|}{ That } \\
\hline & 1905 & I896 & 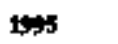 & 190 & ths & IFis \\
\hline 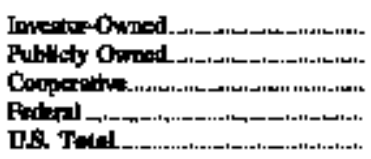 & $\begin{array}{r}6,933 \\
593 \\
67 \\
629 \\
0727\end{array}$ & $\begin{array}{r}5,590 \\
619 \\
94 \\
540 \\
6440\end{array}$ & $\begin{array}{r}1 \\
15 \\
4 \\
10\end{array}$ & $\begin{array}{r}1 \\
8 \\
4 \\
0 \\
13\end{array}$ & $\begin{array}{r}6.9213 \\
609 \\
11 \\
629 \\
8,202\end{array}$ & $\begin{array}{r}5,591 \\
620 \\
99 \\
500\end{array}$ \\
\hline
\end{tabular}

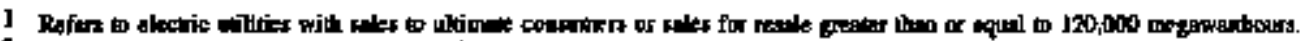

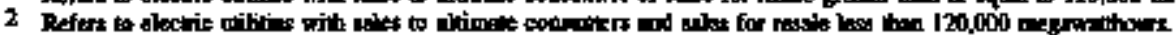

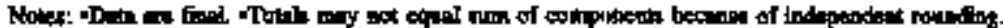

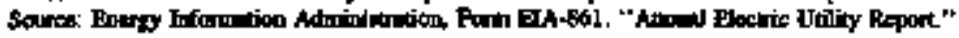

Table 7. U.S. Flectric Uillty Incremental Energy Savings by Program Category, 195 and 1996 (Million Kilowautbours)

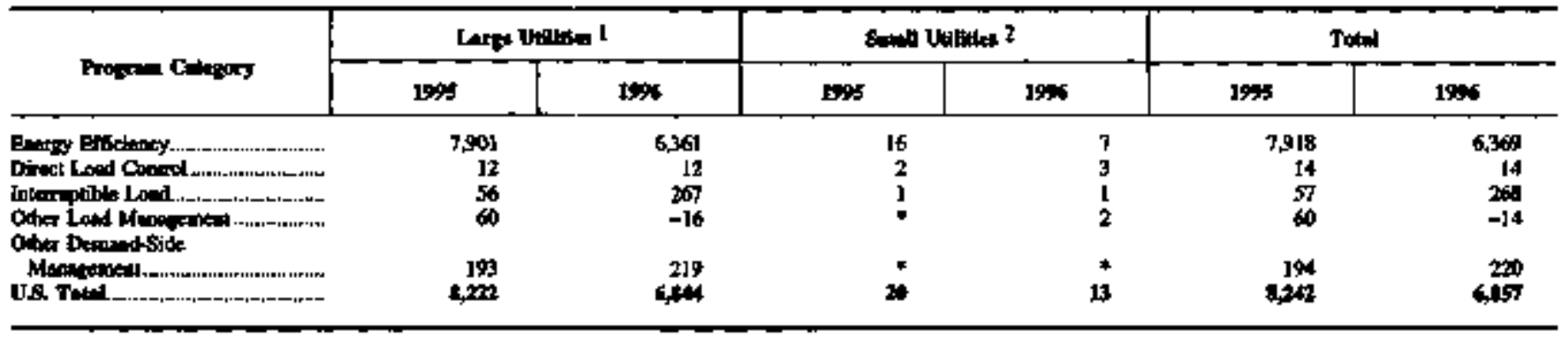

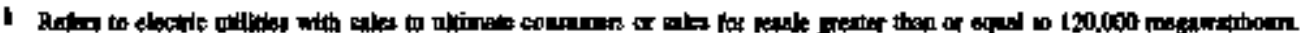

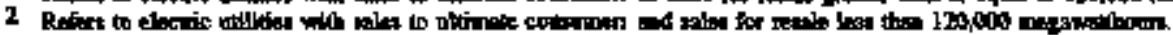

- Valis lass tian. A.s.

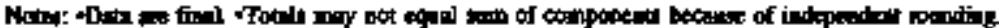

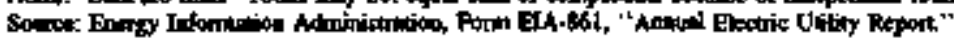

Toble 8. U.S. Electric Utilly Inerenentel Finergy Saving by Sector, 1995 and 1996 (Million Kilowatthours)

\begin{tabular}{|c|c|c|c|c|c|c|}
\hline \multirow{2}{*}{ Sector } & \multicolumn{2}{|c|}{ 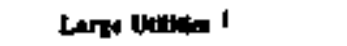 } & \multicolumn{2}{|c|}{ son twing 2} & \multicolumn{2}{|c|}{ Totel } \\
\hline & loss & 1506 & tos & 1926 & נית & 19ws \\
\hline 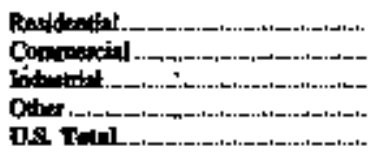 & $\begin{array}{r}1,630 \\
4,594 \\
2,679 \\
320 \\
2,772\end{array}$ & $\begin{array}{r}1,179 \\
3,531 \\
1,781 \\
341 \\
6,844\end{array}$ & $\begin{array}{r}9 \\
5 \\
5 \\
2 \\
2\end{array}$ & $\begin{array}{r}7 \\
3 \\
2 \\
t \\
13\end{array}$ & $\begin{array}{r}1,639 \\
4,599 \\
1,683 \\
311 \\
8,248\end{array}$ & $\begin{array}{r}1,186 \\
3,540 \\
1,789 \\
342 \\
157\end{array}$ \\
\hline
\end{tabular}

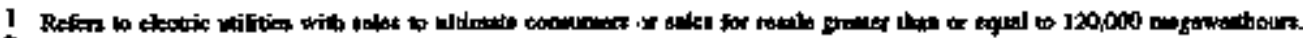

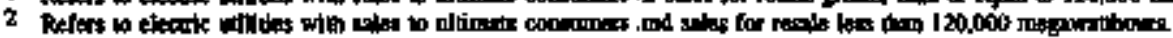

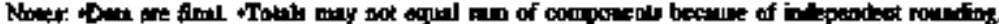

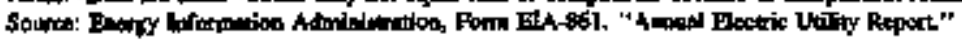


Tahle 9. U.S. Hectric Utiliky Euergy Savings by North Ameriean Electric Reliability Councl Region and Fawnil oy Class of Owerenhy, 1995, 19\%, 19\%7, and 2\%1 (Million Kilowathours)

\begin{tabular}{|c|c|c|c|c|c|}
\hline \multirow{2}{*}{ 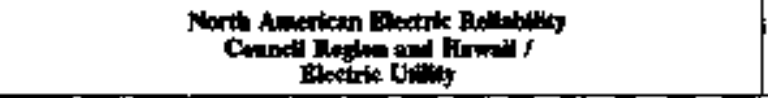 } & \multirow{2}{*}{ קים } & \multicolumn{2}{|c|}{ 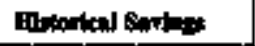 } & \multicolumn{2}{|c|}{ Projected suring } \\
\hline & & 1958 & IFis & & 2011 \\
\hline 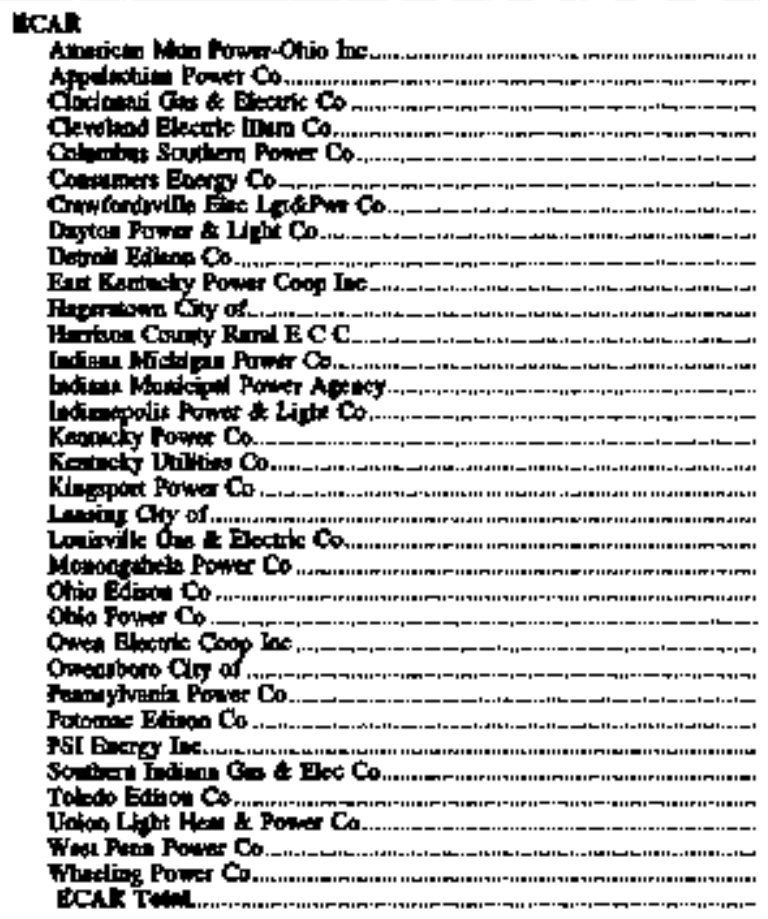 & 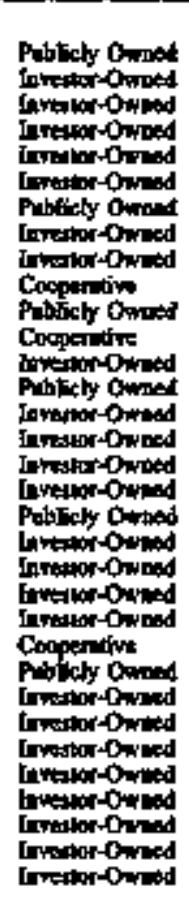 & 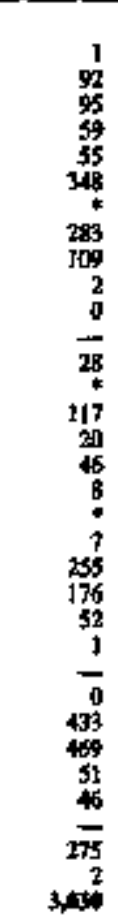 & 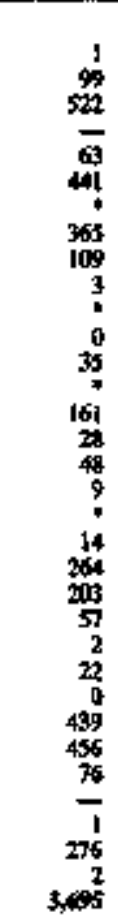 & 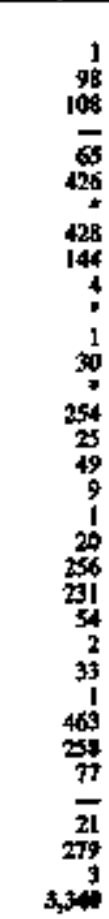 & 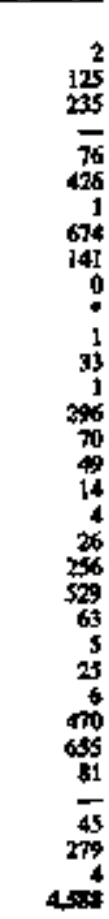 \\
\hline 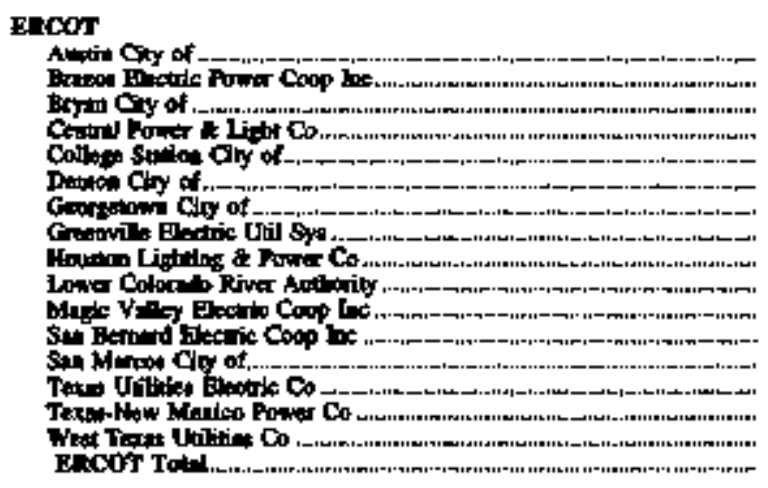 & 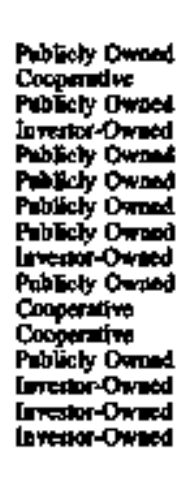 & 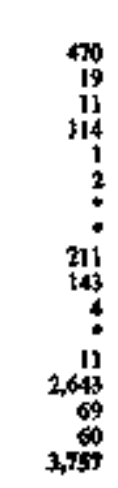 & $\frac{24}{134}$ & 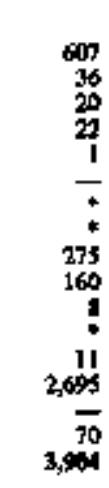 & 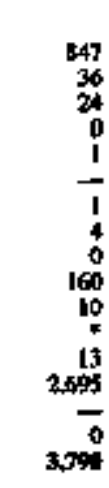 \\
\hline 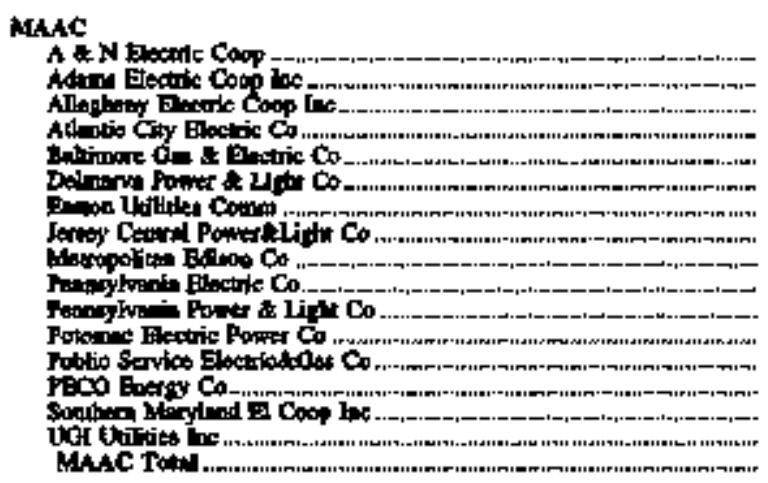 & 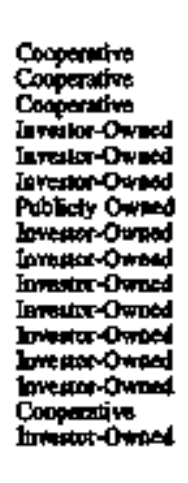 & 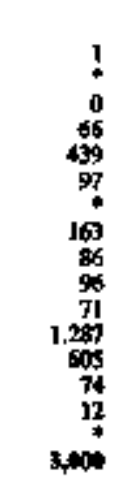 & 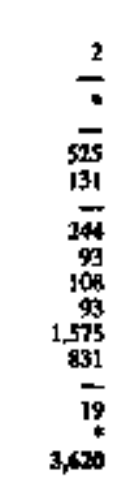 & 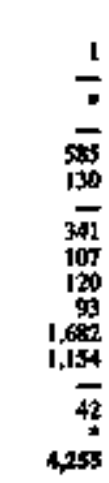 & 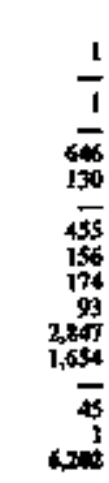 \\
\hline
\end{tabular}

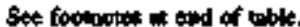


Table 9. U.S. Electric Utility Energy Sevings by North American Blectric Reliahility Cownch Rejion and Howall by Cless of Ownership, 1\%9, 19\%, 1997, and 2001 (Milion Kilowatthours) (Coutinued)

\begin{tabular}{|c|c|c|c|c|c|}
\hline \multirow{2}{*}{ 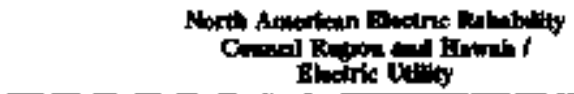 } & \multirow{2}{*}{ omerip at } & \multicolumn{2}{|c|}{ 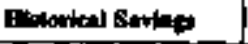 } & \multicolumn{2}{|c|}{ Projecied Smoly } \\
\hline & & Les & 19:4 & 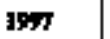 & 201 \\
\hline 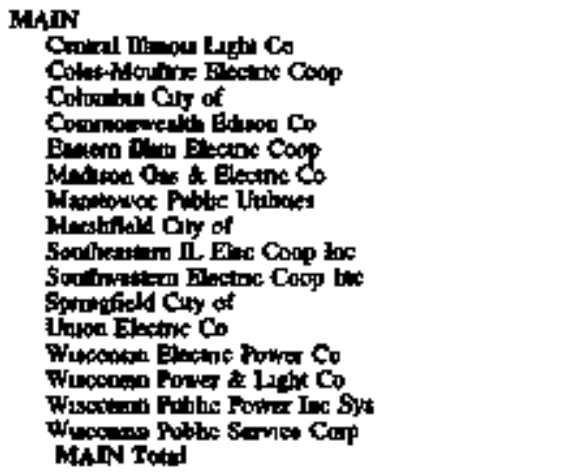 & 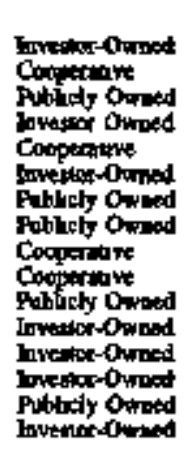 & 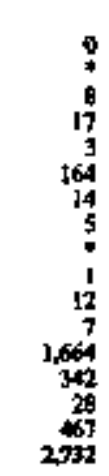 & $\begin{array}{r}1 \\
9 \\
25 \\
3 \\
192 \\
14 \\
5 \\
1 \\
15 \\
15 \\
5 \\
477 \\
376 \\
346 \\
3,45\end{array}$ & $\begin{array}{r}1 \\
4 \\
16 \\
0 \\
3 \\
242 \\
14 \\
5 \\
0 \\
0 \\
19 \\
5 \\
1.842 \\
487 \\
5 \\
504 \\
3,253\end{array}$ & $\begin{array}{r}3 \\
10 \\
0 \\
3 \\
366 \\
15 \\
7 \\
0 \\
0 \\
31 \\
185 \\
1,760 \\
786 \\
3 \\
0 \\
3,17 \%\end{array}$ \\
\hline 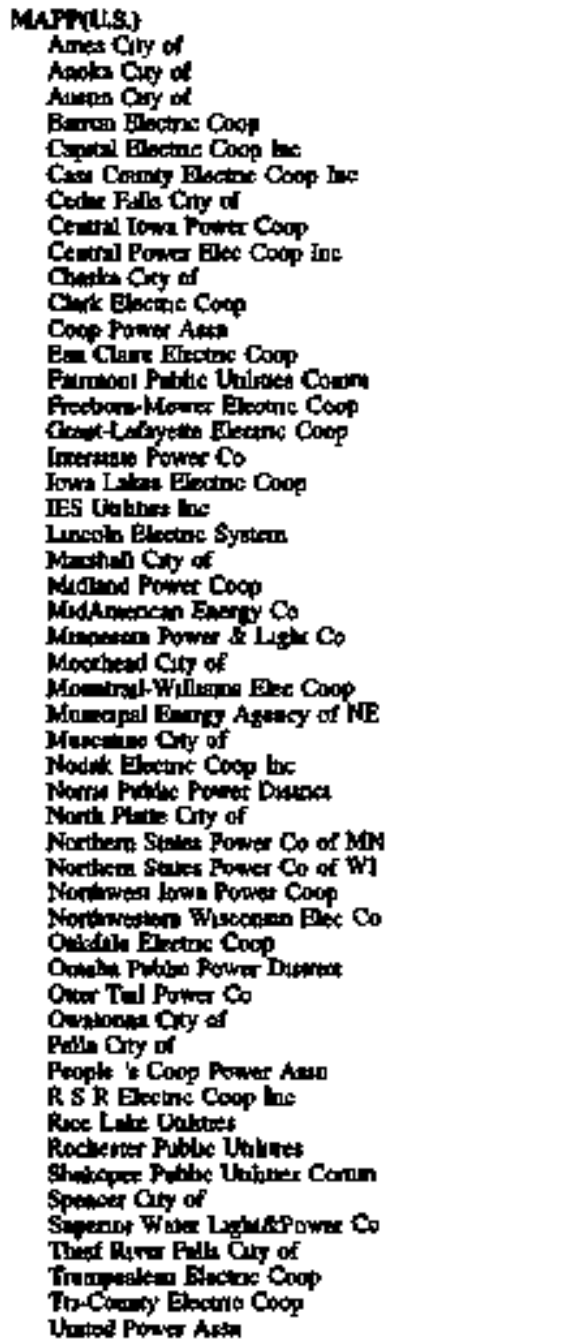 & 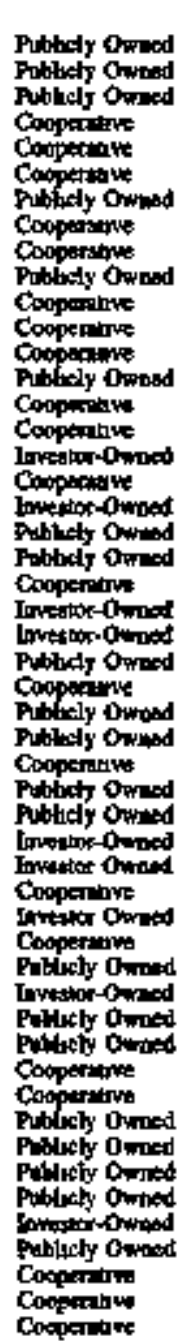 & 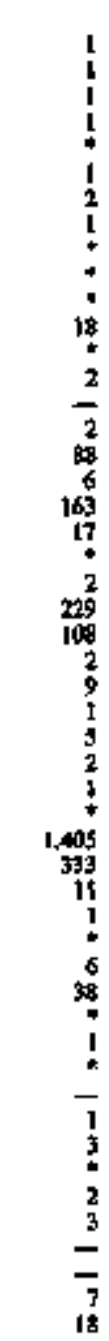 & 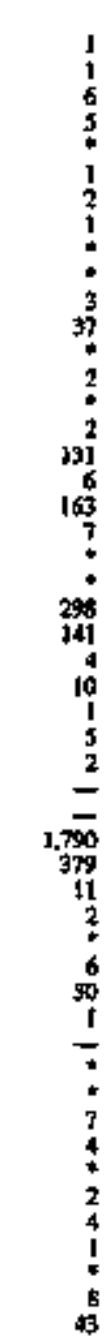 & 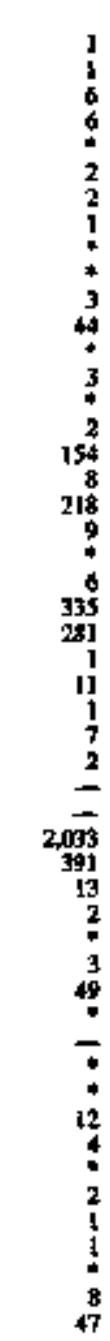 & 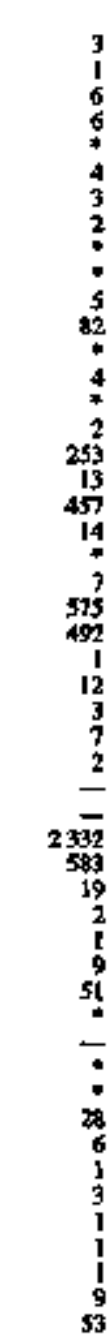 \\
\hline
\end{tabular}

Sec footuntes \# ood of toble 
Table 9. U.S. Electric Utilty Enerdy Savings by North American Blectric Rellabiliky Councll Region and Hawaib by Closs of Owaership, 1995, 1996, 1997, and 2001

(Million Kilowalthours) (Continued)

\begin{tabular}{|c|c|c|c|c|c|}
\hline \multirow{2}{*}{ 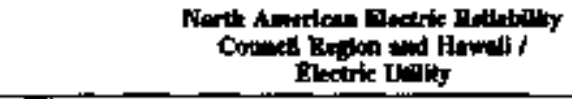 } & \multirow{2}{*}{ Alow ot } & \multicolumn{2}{|c|}{ H[otock' Sonhyge } & \multicolumn{2}{|c|}{ Propeted Sintrog } \\
\hline & & Lss & $19 \%$ & $19 \%$ & $2=1$ \\
\hline 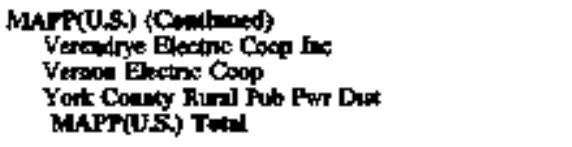 & 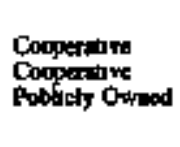 & $\begin{array}{r}2 \\
10 \\
2+4\end{array}$ & $\begin{array}{r}4 \\
10 \\
10\end{array}$ & $\begin{array}{r}4 \\
10 \\
306\end{array}$ & $\begin{array}{r}4 \\
10 \\
506\end{array}$ \\
\hline 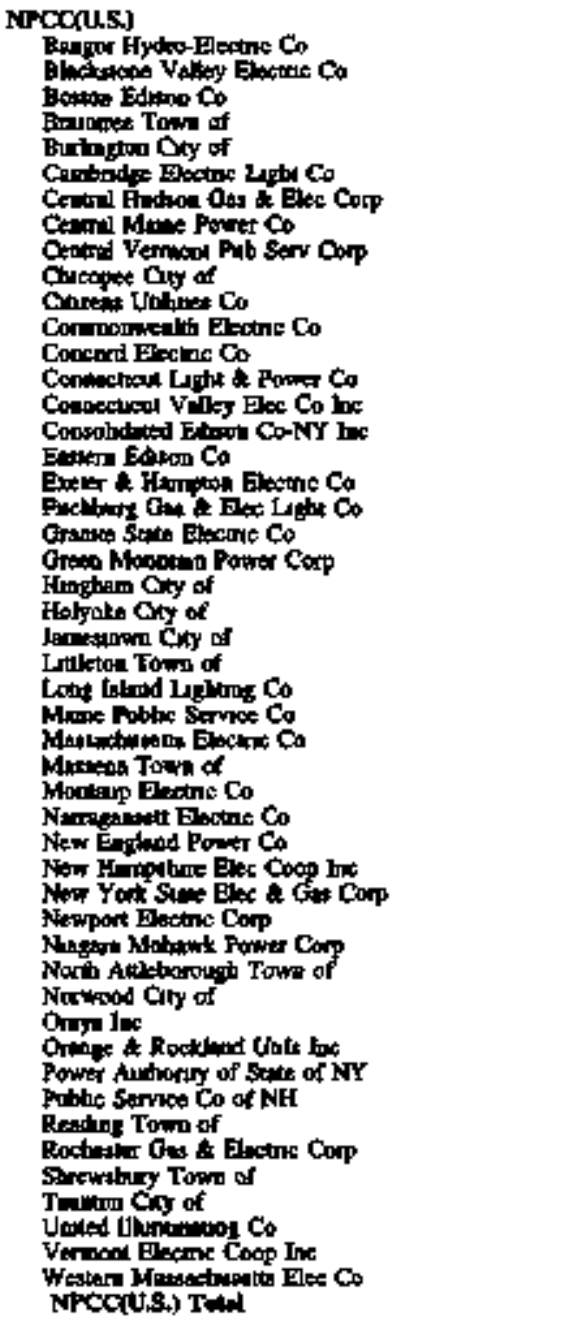 & 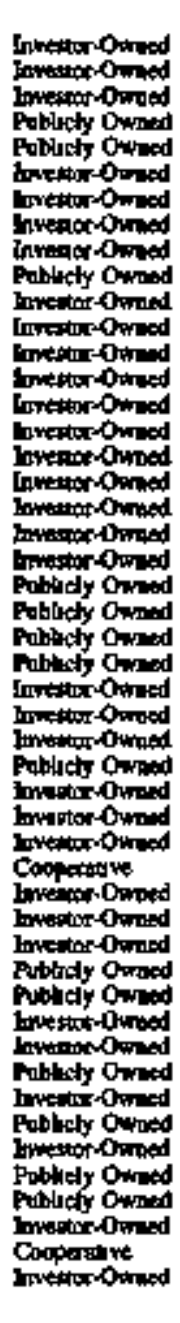 & 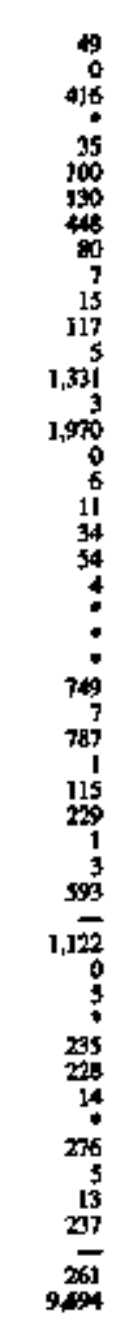 & 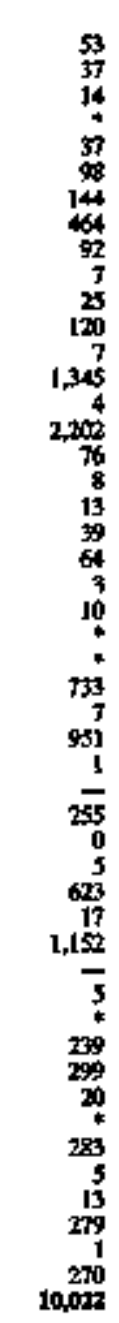 & 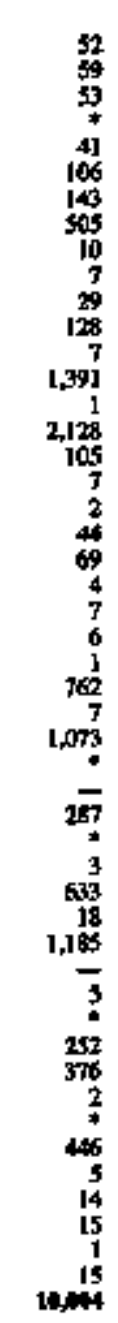 & 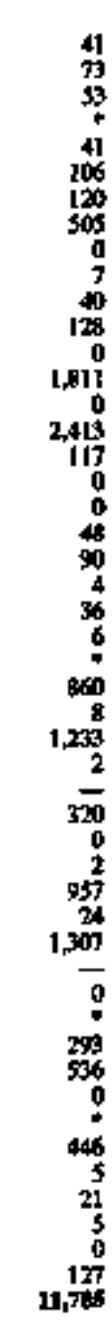 \\
\hline 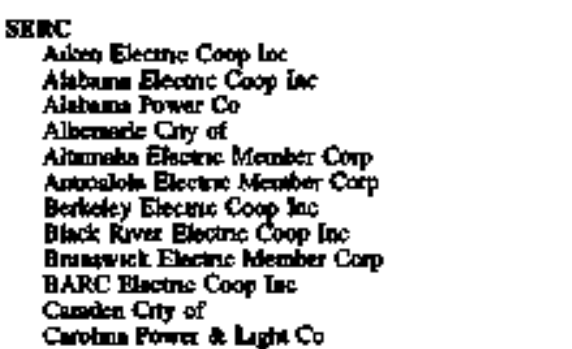 & 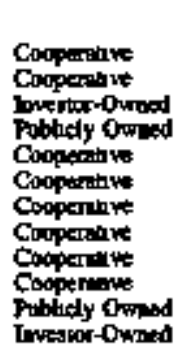 & $\begin{array}{r}1 \\
36 \\
24 \\
4 \\
5 \\
6 \\
2 \\
k \\
0 \\
2,008\end{array}$ & $\begin{array}{r}2 \\
-562 \\
\vdots \\
7 \\
2 \\
0\end{array}$ & $\begin{array}{r}2 \\
4 \\
-601 \\
* \\
7 \\
7 \\
2 \\
0 \\
7 \\
2042\end{array}$ & $\begin{array}{r}2 \\
0 \\
-758 \\
\vdots \\
10 \\
3 \\
0 \\
0 \\
2.162\end{array}$ \\
\hline
\end{tabular}

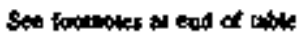


Table 9. U.S. Electric Utity Eneray Suving by North American Electric Reliahility Coancil Reglon and Howall by Class of Owpershtp, 1995, 19\%, 1997, and 2001 (Mitlion Kilowathours) (Continued)

\begin{tabular}{|c|c|c|c|c|c|}
\hline \multirow{2}{*}{ 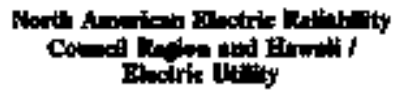 } & \multirow{2}{*}{ on: } & \multicolumn{2}{|c|}{ 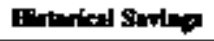 } & \multicolumn{2}{|c|}{ Projeded Sinher } \\
\hline & & 3\% & I\%) & וקצו & 201 \\
\hline
\end{tabular}

S:RC (C) (C)

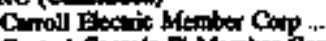

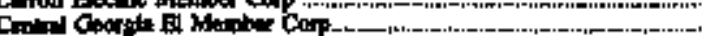

Contal Mirinia Ereatic Coop.

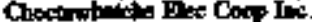

Coutin Eletric Manka Cor

Cowb Dioctic Mmberitip Corp

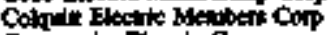

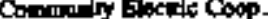

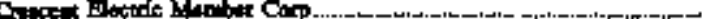

Downtas Cry of

Duk Pow

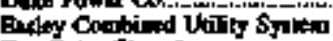

Bist Foir Clo of .

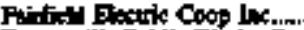

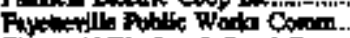

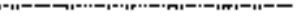

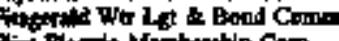

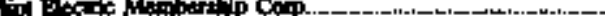

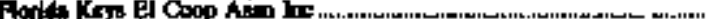

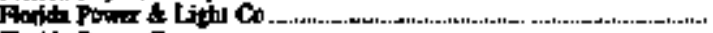

Finidn Powne Cop ...

Fort Pinw Uititis Auth

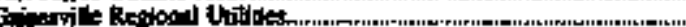

Gearin Mutr Co

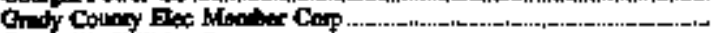

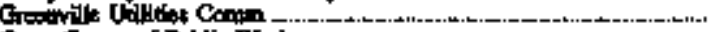

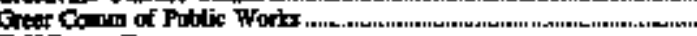

Calle rome Ca

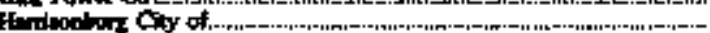

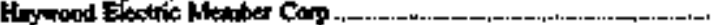

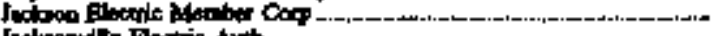

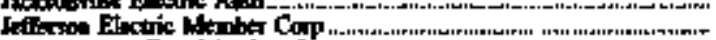

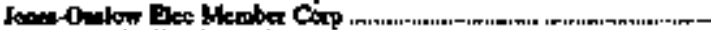

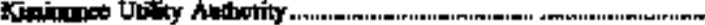

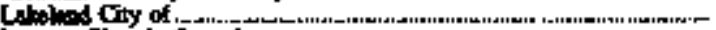

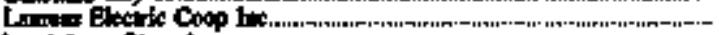

In riplont Cy of

Corractile Got of

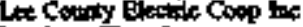

Lecking City of

Lyoches Ave Eloc Coop toc

Nives Gity of

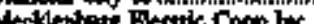

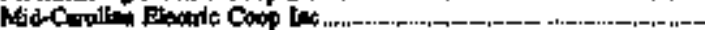

Mhatulupt Powe Co

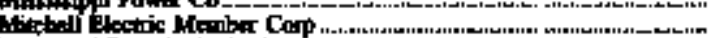

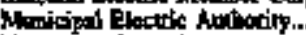

Now Bent Sty of

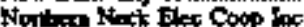

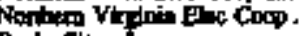

Octols chist.

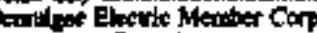

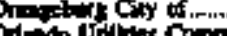

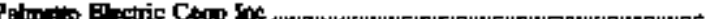

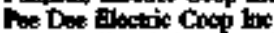

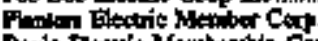

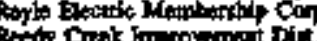

Rock HII Chy of

Rocky Mornt tiky of wurn

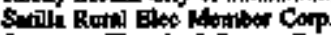

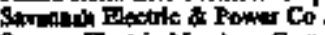

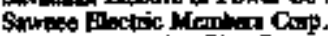

(1)

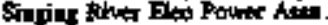

\section{Coopolimintive}

Cooperithe

Cosporative

Cooperative

Cooperndive

Cocpertivive

Copparativt

Coopertivits

Coopormitue

Pofifly Ontad

Inition-Gined

Publicit 0nered

Putioly Onoed

Cocporitive

Coporats:

Pubticly Owisd

Pubincly Oneed

Coopantive

Copperatio

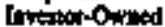

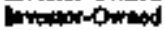

Publity Dimat

Pavikly Onned

Inearo-Owex

Coopentive

Publich 0-ined

Publichty omost

Erreson-0wited

Pavichy Dwned

Cooperitivo

Cooperntive

Pubficty Ontoed

Cocpiritift

Cocostulne

Polity Dwat

Pultaty or

Coopertive

Pubtedy Owed

Publkby Ondod

Cocpotivit

Pubicly Owhed

Putitich owed

Cooperatho

Publisty Otred

Plikely Owned

Cosperative

Cooperathe

Griar-Omed

Cogotative

Publity Onasd

Putiply Omosd

Cospontive

Cocpatiot

Palicly Ouad

Cocpertive

Publikly Omed

Pubich Ond

Cocicentin

Cocpertim

Cotirtive

Congative

robith Oownd

Publely ownsd

Prollely owned

Copprotive

tintomitomed

copparant

Cooperitim

Coppefinitit

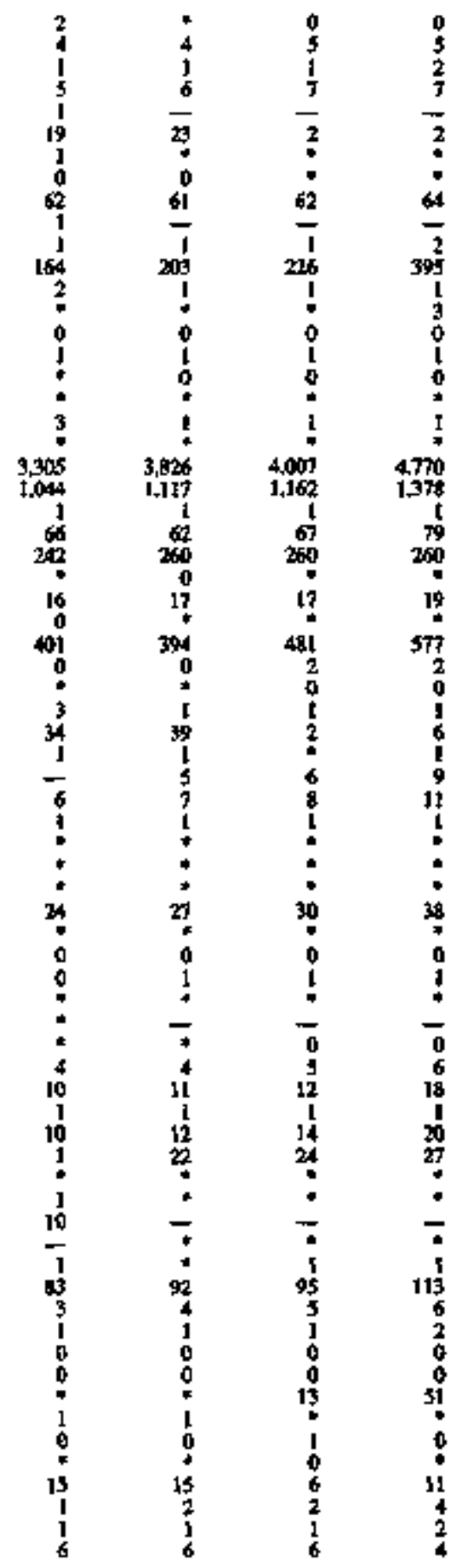

Sea fotomin af end of thite. 
Table 9. U.S. Electric Utility Ruency Sovings ty North Anarican Bectric Reliability Councll Reglon and Hawnil by Clas of Ownershlp, 1995, 1936, 1997, and 2001

Million Kilowatthours) (Continued)

\begin{tabular}{|c|c|c|c|c|c|}
\hline \multirow{2}{*}{ 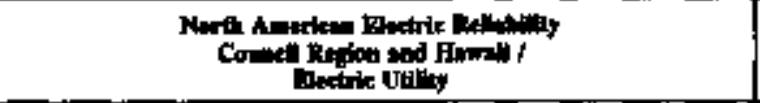 } & \multirow{2}{*}{ Omanctip of } & \multicolumn{2}{|c|}{ Fittorked S-ming } & \multicolumn{2}{|c|}{ Trejeded Sering } \\
\hline & & oss & thos & $t * t$ & II \\
\hline 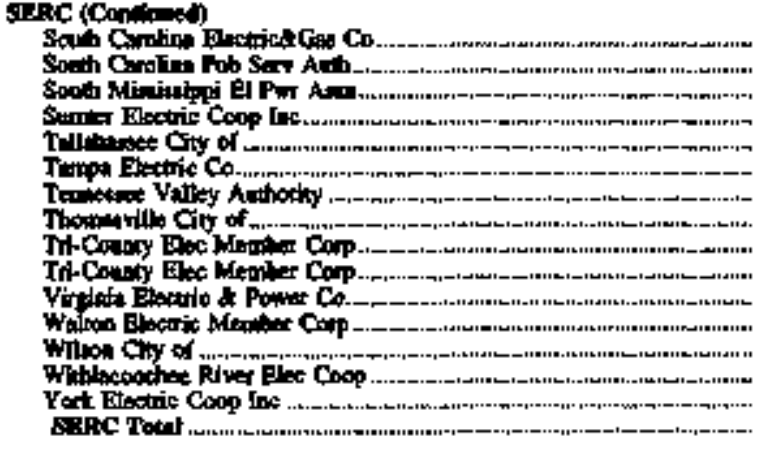 & 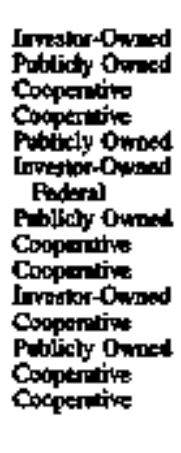 & 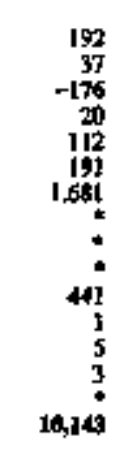 & $\begin{array}{r}194 \\
42 \\
24 \\
27 \\
119 \\
20 \\
1,69 \\
0 \\
0 \\
303 \\
\frac{7}{4} \\
10,44\end{array}$ & $\begin{array}{r}205 \\
49 \\
33 \\
21 \\
127 \\
265 \\
1,713 \\
0 \\
386 \\
6 \\
18 \\
14.96\end{array}$ & $\begin{array}{r}216 \\
80 \\
55 \\
27 \\
164 \\
424 \\
1,400 \\
4 \\
0 \\
410 \\
7 \\
7 \\
28 \\
12,4\end{array}$ \\
\hline 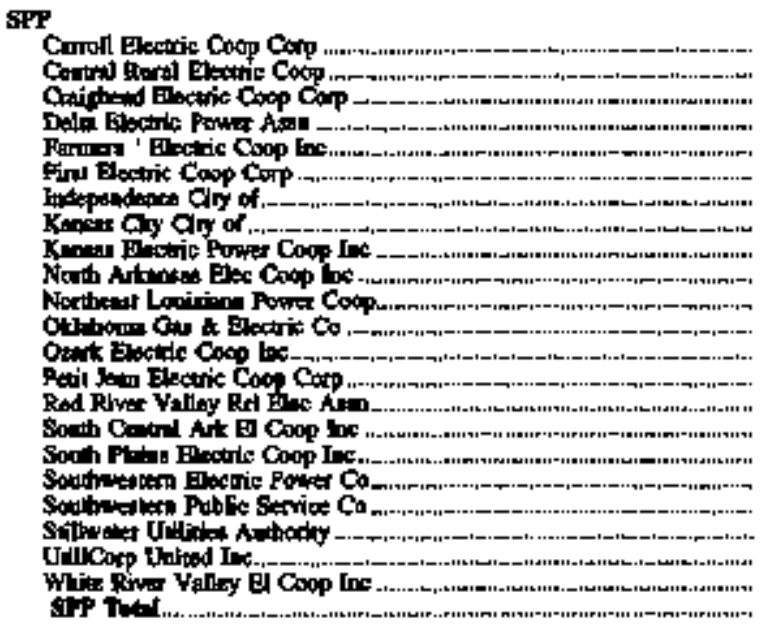 & 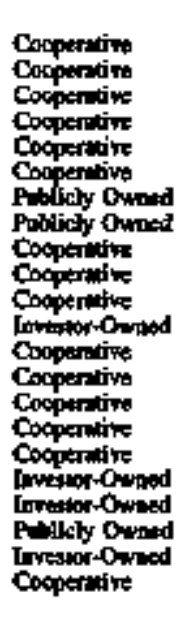 & 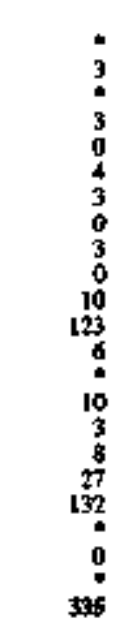 & 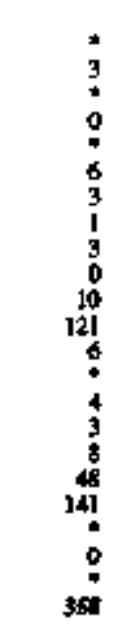 & 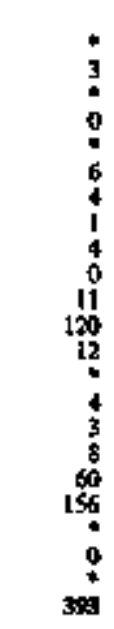 & 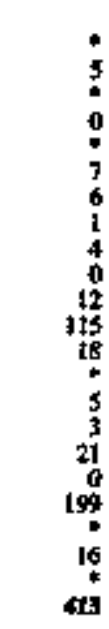 \\
\hline 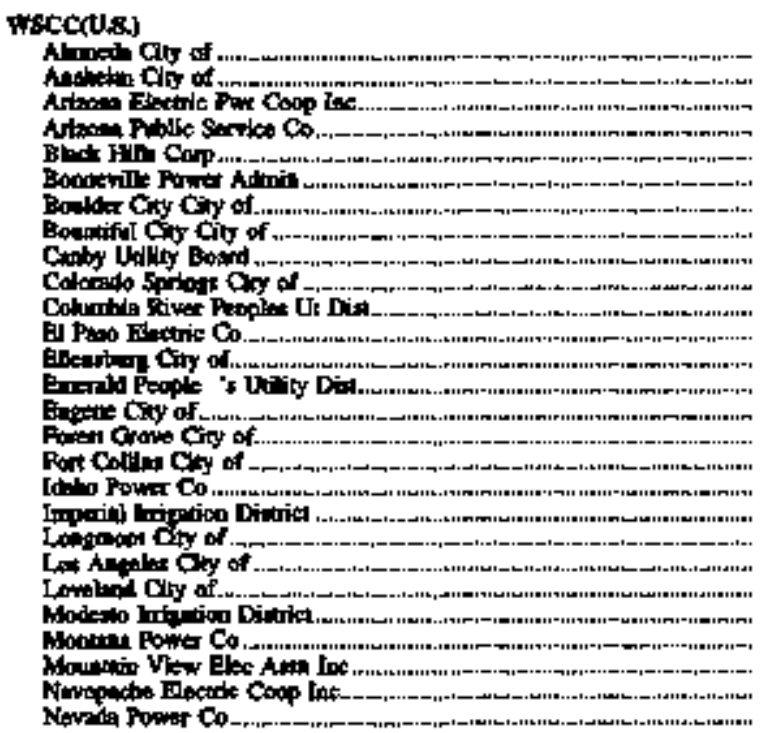 & 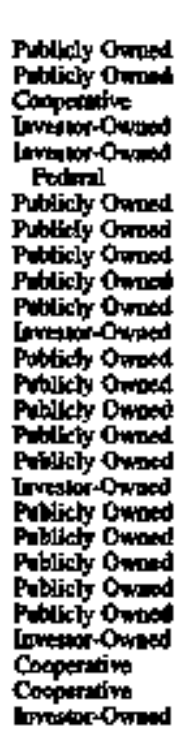 & 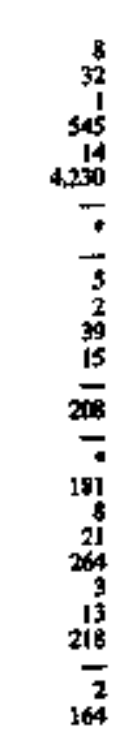 & $\begin{array}{r}10 \\
37 \\
0 \\
545 \\
475 \\
6 \\
4 \\
4 \\
6 \\
39 \\
15 \\
12 \\
231 \\
8 \\
0 \\
185 \\
9 \\
16 \\
273 \\
3 \\
14 \\
290 \\
6 \\
151\end{array}$ & $\begin{array}{r}11 \\
39 \\
0 \\
566 \\
5,124 \\
7 \\
1 \\
5 \\
7 \\
10 \\
16 \\
17 \\
250 \\
15 \\
0 \\
199 \\
9 \\
27 \\
273 \\
15 \\
265 \\
151\end{array}$ & $\begin{array}{r}5 \\
86 \\
6 \\
374 \\
5,711 \\
13 \\
1 \\
40 \\
10 \\
18 \\
17 \\
350 \\
13 \\
0 \\
213 \\
10 \\
26 \\
205 \\
4 \\
4 \\
46 \\
4 \\
3 \\
75\end{array}$ \\
\hline
\end{tabular}

Sec foomotes a and of chold. 


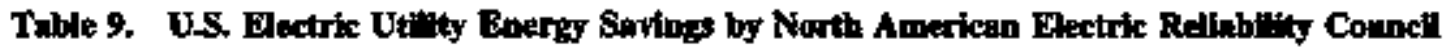
Region and Hawall by Close of Ownerstip, 1955, 1996, 1997, and 2001

(Million Kilowatthours) (Continued)

\begin{tabular}{|c|c|c|c|c|c|}
\hline \multirow{2}{*}{ 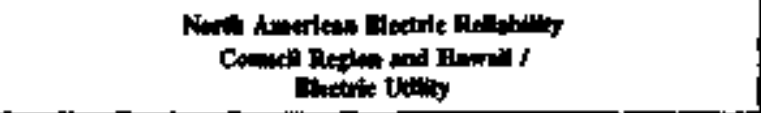 } & \multirow{2}{*}{ Oence of } & \multicolumn{2}{|c|}{ 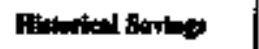 } & \multicolumn{2}{|c|}{ Projected Stringy } \\
\hline & & $15 \%$ & Ins & J & 2:11 \\
\hline 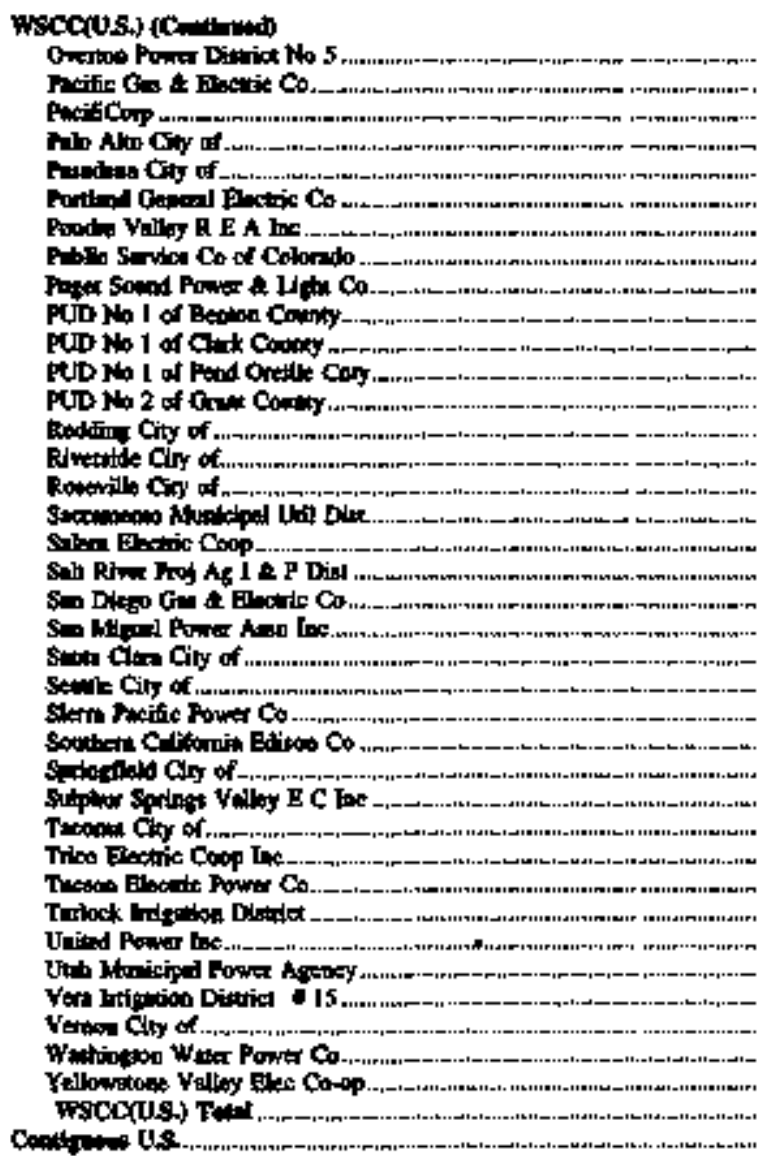 & 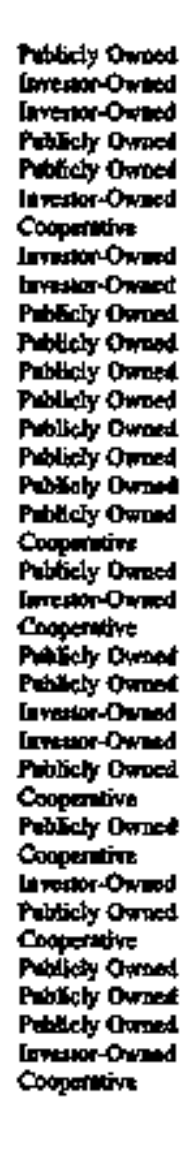 & 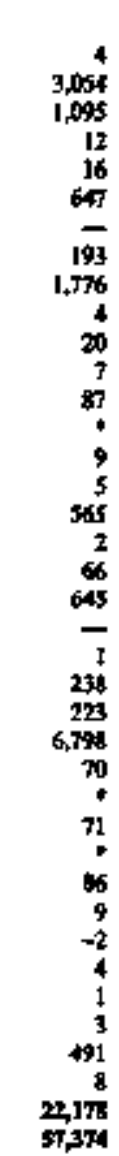 & 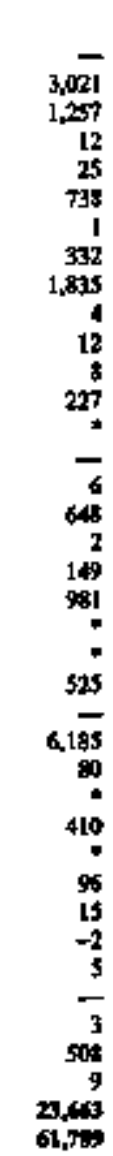 & 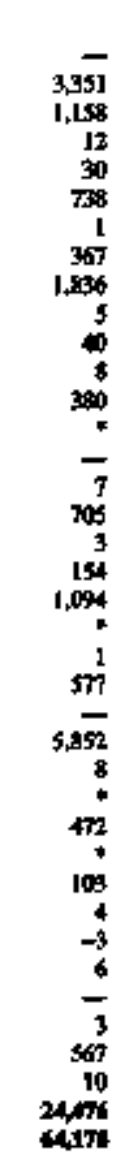 & 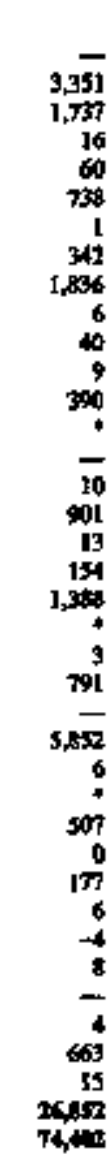 \\
\hline 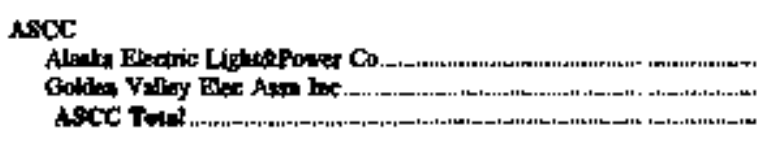 & 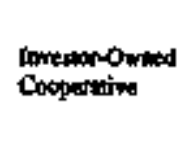 & $\dot{4}$ & : & $\begin{array}{l}0 \\
5 \\
5\end{array}$ & \\
\hline 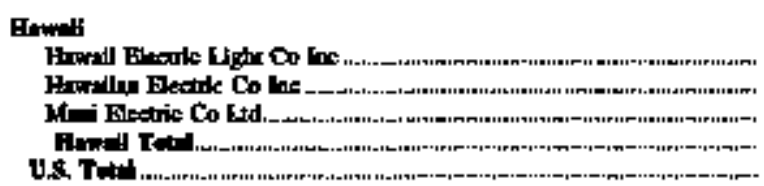 & 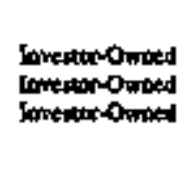 & $\begin{array}{r}13 \\
29 \\
40,19\end{array}$ & $\begin{array}{r}12 \\
28 \\
49\end{array}$ & $\begin{array}{r}3 \\
30 \\
30\end{array}$ & $\begin{array}{r}71 \\
0 \\
75 \\
140\end{array}$ \\
\hline
\end{tabular}

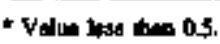

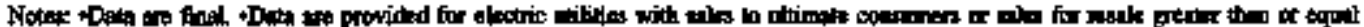

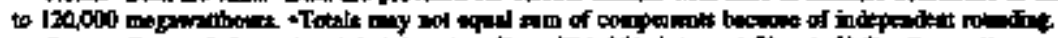

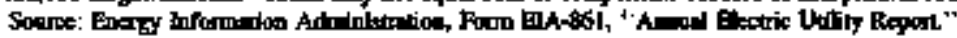




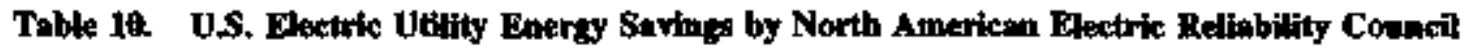
Region and Eawali by DSM Program Category, 1996

(Million Kilowatrhours)

\begin{tabular}{|c|c|c|c|}
\hline 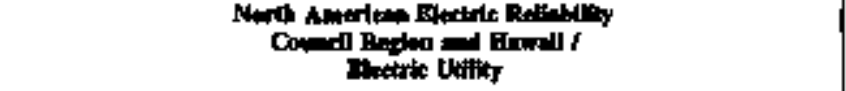 & Buctipy & 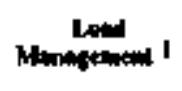 & Trad DSM \\
\hline 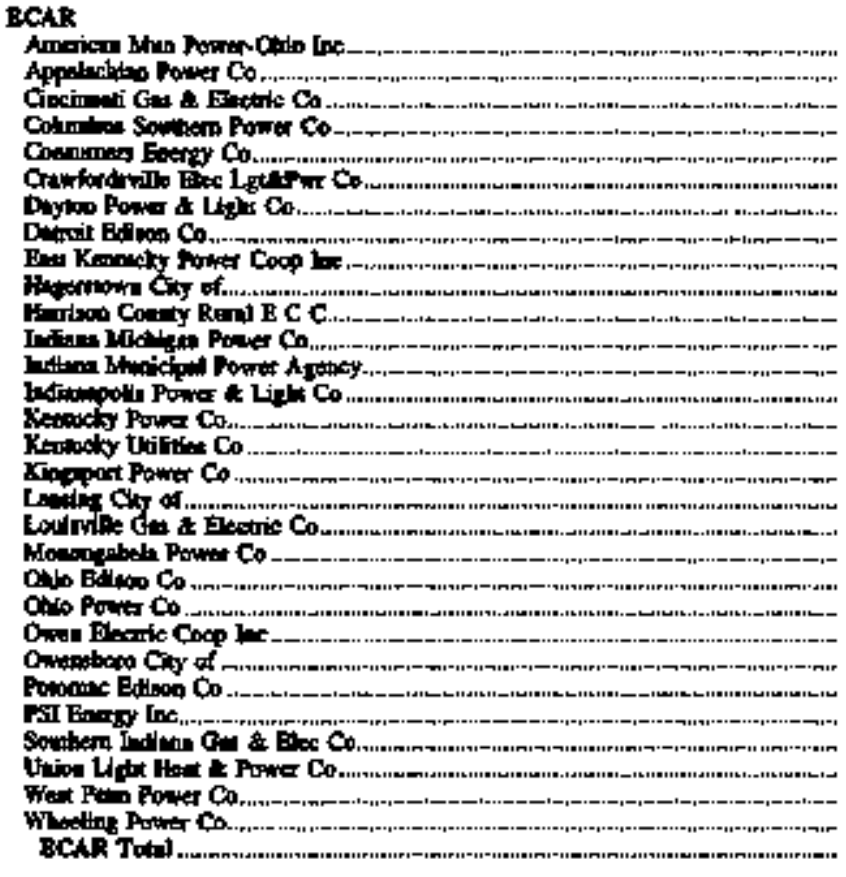 & 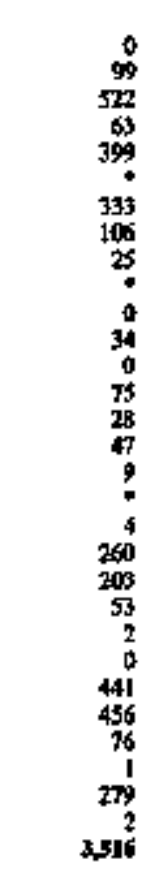 & $\begin{array}{r}1 \\
0 \\
0 \\
42 \\
0 \\
32 \\
3 \\
-22 \\
0 \\
5 \\
2 \\
80 \\
4 \\
7 \\
0 \\
0 \\
10 \\
5 \\
4 \\
4 \\
0 \\
22 \\
-1 \\
0 \\
0 \\
0 \\
-3 \\
179\end{array}$ & $\begin{array}{r}1 \\
99 \\
527 \\
63 \\
441 \\
4 \\
365 \\
109 \\
3 \\
35 \\
161 \\
29 \\
48 \\
9 \\
14 \\
264 \\
209 \\
57 \\
2 \\
27 \\
439 \\
456 \\
76 \\
1 \\
276 \\
2 \\
3495\end{array}$ \\
\hline 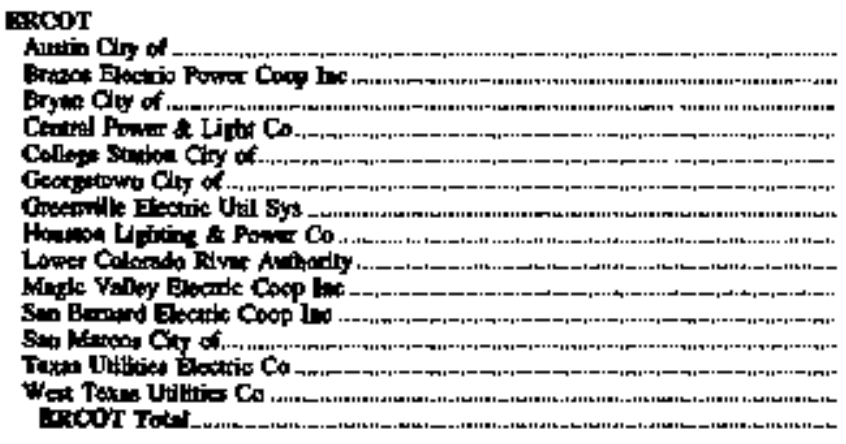 & 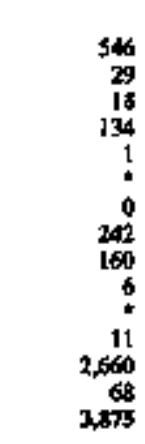 & $\begin{array}{r}0 \\
0 \\
0 \\
0 \\
2 \\
0 \\
F \\
-10 \\
0 \\
0 \\
0 \\
0 \\
0 \\
0 \\
-0\end{array}$ & $\begin{array}{r}546 \\
29 \\
15 \\
134 \\
2 \\
5 \\
232 \\
160 \\
6 \\
11 \\
2,60 \\
60 \\
3 \times 65\end{array}$ \\
\hline 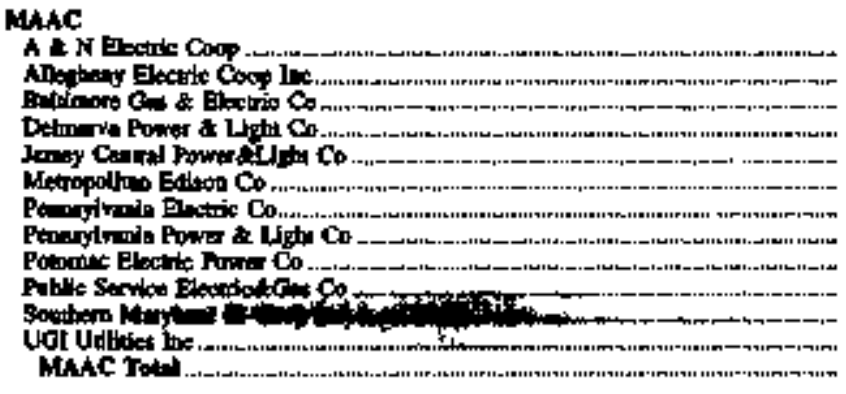 & $\begin{array}{r}1 \\
525 \\
131 \\
244 \\
75 \\
100 \\
93 \\
1,43 \\
831 \\
19 \\
3,451\end{array}$ & $\begin{array}{r}1 \\
0 \\
0 \\
0 \\
18 \\
7 \\
0 \\
142 \\
0 \\
0 \\
0 \\
180\end{array}$ & $\begin{array}{r}2 \\
523 \\
131 \\
244 \\
93 \\
108 \\
93 \\
155 \\
83 ! \\
19 \\
396\end{array}$ \\
\hline 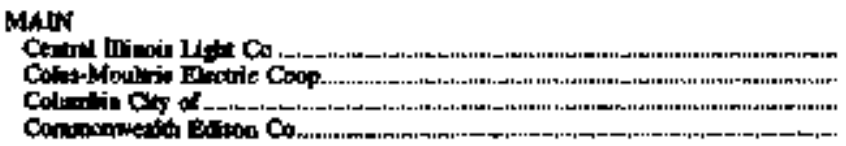 & $\begin{array}{r}1 \\
0 \\
7 \\
24\end{array}$ & : & $\begin{array}{r}1 \\
9 \\
25\end{array}$ \\
\hline
\end{tabular}

See footnoties of ead of whe. 


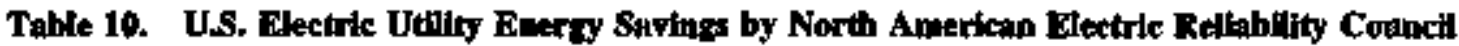
Rejion and Hanadi by DSM Frogran Category, 1 ss (Millon Kilowatthours) (Continued)

\begin{tabular}{|c|c|c|c|}
\hline 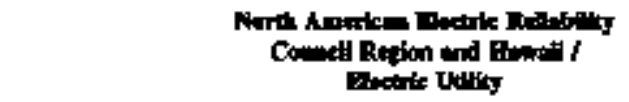 & Dowas & 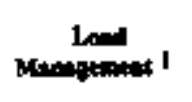 & $\begin{array}{l}\text { Told DSM } \\
\text { Progrtin: }\end{array}$ \\
\hline
\end{tabular}

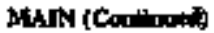

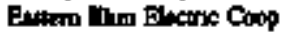

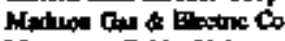

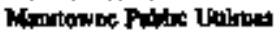

Mratifid Cing of

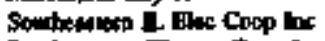

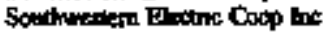

Spanofiold Ciry of

Unan Jacke Co

Wractoro Eleotic Rowar Co

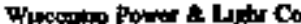

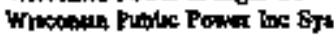

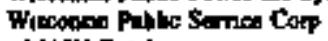

MAN THA

\section{MAFirtus}

Ames Cxy of

Angla Cxy of

Nom Cing of

Binot Eletinc Coos

Cupte' Elatax Coch b:

Cun Conny Eiecter Coop in

Cedar Fills $\mathrm{Cr}$, of

Cenial low Pown Coop

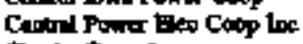

Chish Coty of

Cinct Blectric Cos

Cowp forret hes

Bin Dlane Elwone Doop

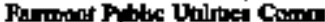

Fetbon-More Electrile Coop

Gra-[afoyous blectere Coop

Interotents Poser Co

Imwa Lales Beatinc Coop

IFS Ualuing ins

Lupcoln Elontinc Sydeta

Mental. Cty at

Mrind Rower Cocp

Mudhmetncat Eersy Co

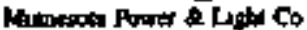

Moctiond Cuy of

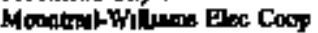

Monstial Eotrey Araney of NE

Mutcians City of

Modink Eloconc Coop Inc

Nhathere Stand Pown Co of MDN

Northore 5atis loom Co of WT

Nontuma bous Powa Coop

Nortumberse Wuponan Flec Co

O-tidale Fatinc Coop

On:uk Pobbe Powr Dumet

Onter Trol Pumar Co

Owatonen Cy of

Fexplo " Coop Pown Am

R \& $R$ toctsc Coop In

Ruac Listic Uhative

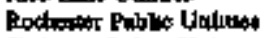

Shatrope Pabuc Undrtes Comn

Sen footortes 미 and of th

$\begin{array}{rrr}1 & 2 & 3 \\ 192 & 0 & 192 \\ 14 & 0 & 14 \\ 5 & 0 & 5 \\ 0 & 5 & 4 \\ 0 & 1 & 1 \\ 5 & 0 & 15 \\ 0 & 5 & 5 \\ 1.724 & 13 & 1,757 \\ 417 & 0 & 417 \\ 35 & 1 & 36 \\ 543 & 3 & 546 \\ 2,739 & 25 & 3,45\end{array}$

\begin{tabular}{|c|c|c|}
\hline I & 0 & 1 \\
\hline I & • & $t$ \\
\hline 5 & 1 & 6 \\
\hline I & 3 & 5 \\
\hline 0 & • & * \\
\hline I & 1 & I \\
\hline 2 & 0 & 2 \\
\hline I & 0 & 1 \\
\hline 0 & + & + \\
\hline 0 & • & * \\
\hline • & 3 & 3 \\
\hline 34 & 2 & उो \\
\hline$\star$ & 中 & $\bullet$ \\
\hline 0 & $z$ & 2 \\
\hline$*$ & 0 & * \\
\hline • & 2 & 2 \\
\hline 131 & 0 & 131 \\
\hline 6 & ] & 6 \\
\hline 185 & $-t s$ & 163 \\
\hline$T$ & 0 & 7 \\
\hline 0 & $\bullet$ & • \\
\hline$\bullet$ & a & " \\
\hline 293 & 5 & 298 \\
\hline$|4|$ & 0 & 741 \\
\hline 4 & $\star$ & 4 \\
\hline I & 10 & 10 \\
\hline t & $\bullet$ & I \\
\hline 3 & 0 & 5 \\
\hline 0 & 2 & 2 \\
\hline .772 & IB & 1.790 \\
\hline 320 & 51 & 379 \\
\hline It & $\theta$ & 11 \\
\hline 2 & 0 & 2 \\
\hline • & $\bullet$ & * \\
\hline 6 & 0 & 6 \\
\hline 45 & 4 & 50 \\
\hline 0 & I & $\mathbf{1}$ \\
\hline 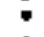 & * & 4 \\
\hline 0 & $\bullet$ & $\star$ \\
\hline 7 & 0 & 3 \\
\hline 3 & I & 4 \\
\hline$\bullet$ & 4 & - \\
\hline
\end{tabular}


Table 10. U.S. Blectric Utility Enerty Savings by North American Blectrte Reliohility Connell Region and Howati by DSM Program Cotezory, 1996

(Million Killowatthours) (Contimued)

\begin{tabular}{|c|c|c|c|}
\hline 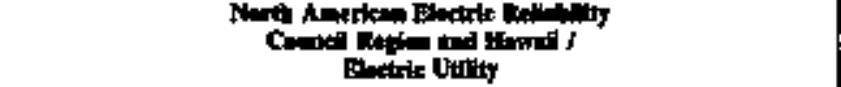 & Enerity & Lowd & \\
\hline 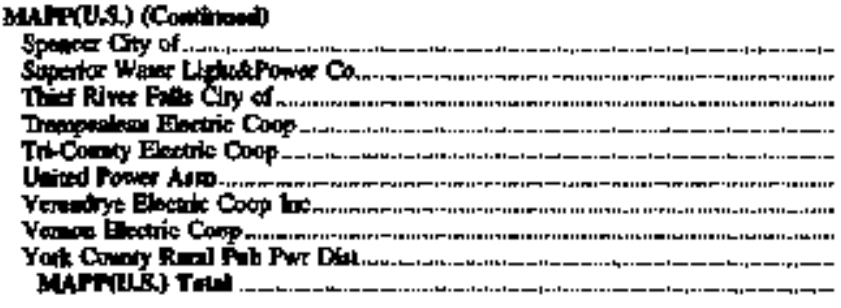 & & $\begin{array}{r}0 \\
0 \\
1 \\
0 \\
1 \\
1 \\
10 \\
10\end{array}$ & $\begin{array}{r}2 \\
4 \\
8 \\
43 \\
10 \\
3 \\
3,+51\end{array}$ \\
\hline 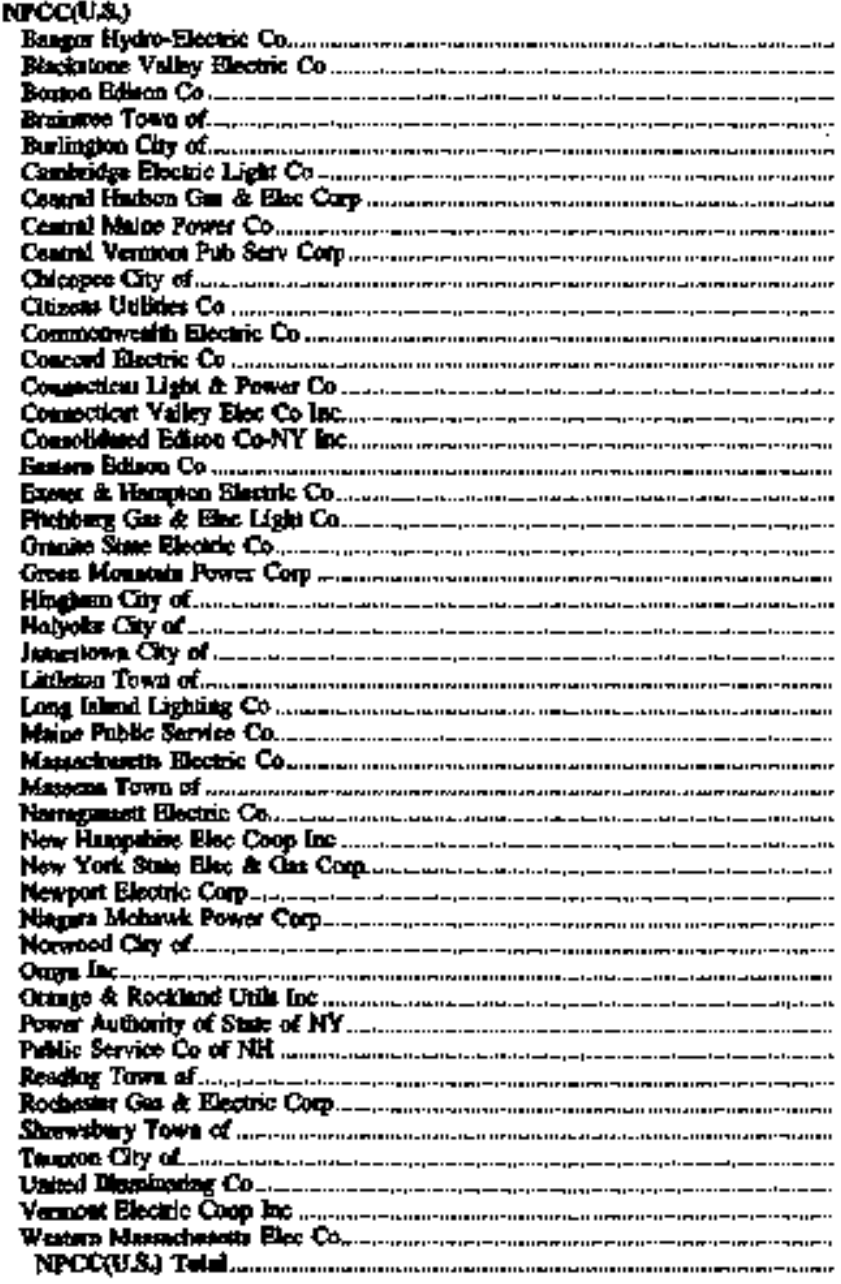 & 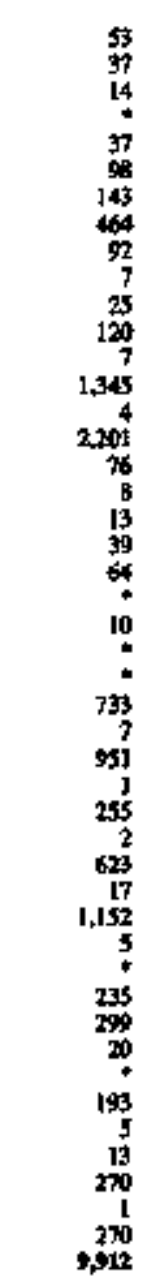 & $\begin{array}{r}0 \\
0 \\
0 \\
0 \\
0 \\
0 \\
0 \\
0 \\
0 \\
0 \\
0 \\
0 \\
0 \\
0 \\
0 \\
0 \\
0 \\
0 \\
0 \\
3 \\
0 \\
0 \\
0 \\
0 \\
1 \\
0 \\
0 \\
0 \\
0 \\
0 \\
0 \\
0 \\
0 \\
0 \\
0 \\
0 \\
0 \\
0 \\
0 \\
0\end{array}$ & 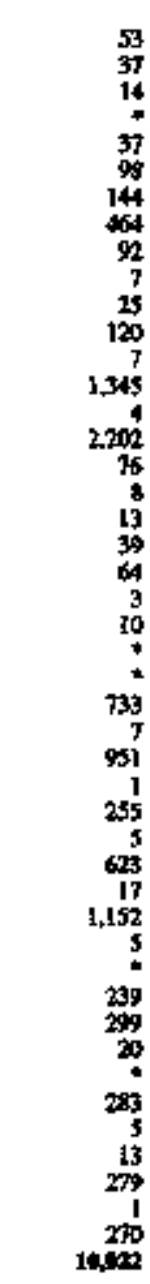 \\
\hline 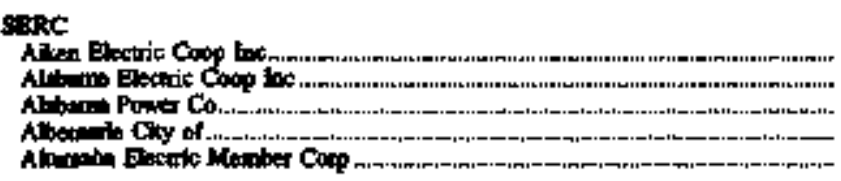 & 2 & $\begin{array}{r}4 \\
-54 \\
+\end{array}$ & $\begin{array}{r}2 \\
-56 \\
*\end{array}$ \\
\hline
\end{tabular}

Sec somoteat at en of the. 
Table 10. U.S. Electric Utility Fnergy Savings by Nortb American Electric Reliabilty Cound Region and Hawall by DSM Program Catetory, 1996

(Million Kilowatthours) (Continued)

\begin{tabular}{|c|c|c|c|}
\hline 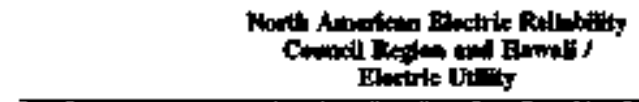 & Binters & 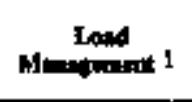 & Tutal Disis \\
\hline
\end{tabular}

SSRC (C - butud)

Ampula Dlectur Member Corp

Baftedey Electre Coop bis

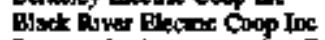

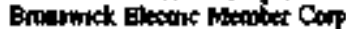

BARC BDanc Coop las

Comida Ciry of

Colma Pun \& La

Cimol Elexnc M.m.

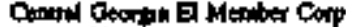

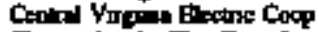

Chochintertar Ekoc Cocp Isc

Cobb Fiectar Membertif Corp

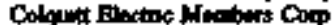

Consury Bextat Coop

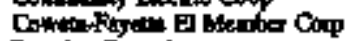

Doogim Cry of

Duts Power Co

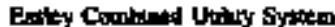

Bat Pomt Oty of

Excolan Fiectic Mender Com

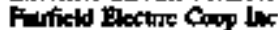

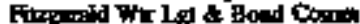

Fin Electene Menberthp Corp

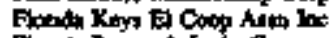

Fianda power \& Lish co

Flond Potror Com

Font Puator Uhbots Anth

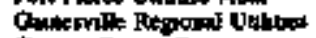

Geortin Pons $\mathbf{C} 0$

Gindy Condy Bree Meriber Cor

Geremile Lation Cos I

Grear Coms of Pobte wests

Gut Powe Co

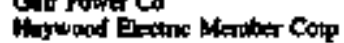

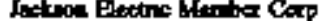

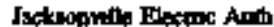

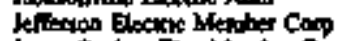

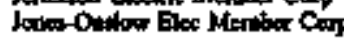

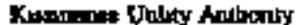

Levolund Cly of

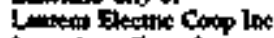

Len

Larrencevilite Con of

Leo County Elotric Coop lox

Lentount chy of

Lombetion Cxy of

Lyonten Ruar Bles Coop Inc

Manetis Oty $x$

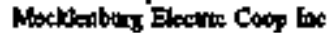

Mud-Carolina Biectra Cosp he

Mesabipin Powar Co

Malchell Ginone Homber Car

Mramapol Elastroc Aupisinty

$N=$ Ben Git of

Worthert Noct Eke cosp toc

Nocthare Vor mus Elec Coop

Ocronget Eloctnc Member Corp

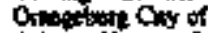

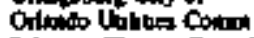

Palrete Finctex Coop loc

Pue Dex Butioc Cocp ho

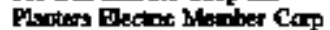

Raylo Eletow Mambarchyp Copp

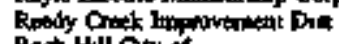

Ract Hall ory of

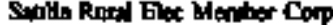

Sinvingat Bectros \& Paner of

Sect tootnotes ax end of tidite 
Twhle 10. US. Electric Utility Eneray Suving by North Anetican Klectric Reliability Coancil Region and Hawail by DSM Program Category, 1996 (Million Kilowatthours) (Continued)

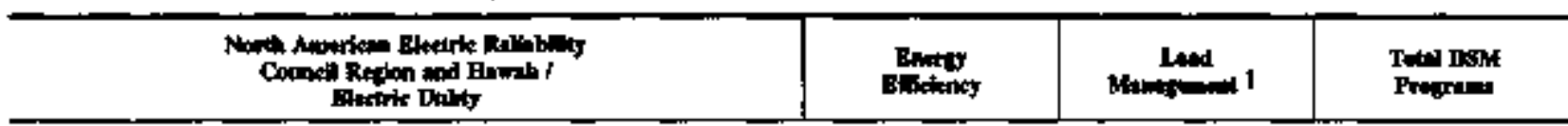

SaRC (Centhed)

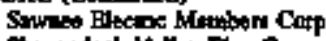

Shontudent Villat Elec Coop

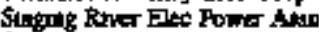

South Crolus Dectucksas Co

Sonch Cinolm phb Serv Auth

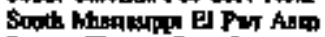

Shinier Electure Coop inc

Fathustory of

Tampe Elocinc Co

Tenpepese Valley Autwong

Ihomatille Crty of

Tn-Condty Elex M-nher Com

Th-Can'y Plx Merabe Corp

Vegron Electic a Powa $\mathrm{Co}$

Wiliow City of

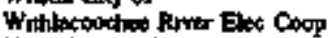

Yot Bfectac Coep lnc

Sanc Tous

sip

Chroll Eletark Coop Cocp

Centril Rurril Elesent Coop

Gruplad Ekente coop onp

Furbet " rectrit coop he

Purst Dectue Coop Coop

Indopendeace City of

Kanis Ony Ciry of

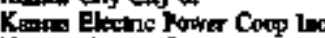

North Nytrois Eloc Coop Inc

Northes: Lans ma Rruer Cow

OHahoma Gan a Eletnc Co

Oent Elowe Coog bac

Pats Jin Elocte coop Cap

Red Ruret Velley Rid Blec Assa

South Cenirad Ait 召 Coop Ine

South Plares Electone Coop he

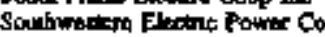

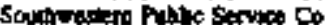

Snllwiver Utilites Auchorion

Whe Rror Valley E Coep Ine ElPP Twel.

wscos(us)

Alvind Coty of

Antwes Cary of

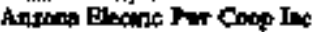

Ansona Pable Sexve Co

Banevile Power Adtan

Boulder Cisy Cy of

Bowath Coty tity of

Comby Vintity Boerd

Cotoraide Spnota Dey of

Colluninge Ruver Peopies Ut Dust

D Paso Electre Co

Dinistare Ory of

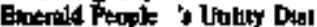

Eopowe Gity of

Forex Grove Ciny of

Ldroto Powe Ca

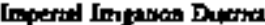

Langment bivy of

Ls Anople City of

Loveland Cxy of

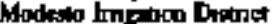

Momane Poner Co

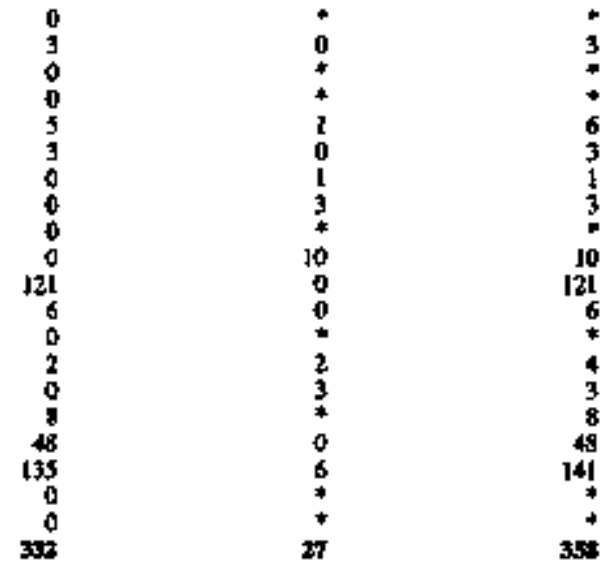

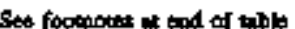

\begin{tabular}{|c|c|c|}
\hline 10 & & \\
\hline 31 & 6 & 37 \\
\hline & 0 & $*$ \\
\hline 545 & 0 & 545 \\
\hline 1878 & 830 & 4,756 \\
\hline & 0 & 6 \\
\hline 中 & 0 & * \\
\hline - & 0 & * \\
\hline 6 & 0 & 6 \\
\hline 23 & 16 & 39 \\
\hline 15 & 0 & 15 \\
\hline 12 & 0 & 12 \\
\hline$\overline{\mathbf{3 1}}$ & 0 & 21 \\
\hline B & 0 & t \\
\hline 135 & 0 & 185 \\
\hline & 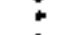 & 9 \\
\hline 3 & 13 & 16 \\
\hline 273 & 0 & 273 \\
\hline & 3 & 3 \\
\hline 14 & 0 & 14 \\
\hline 250 & 0 & 250 \\
\hline
\end{tabular}




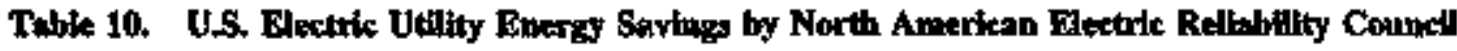
Region and Hawaí by DSM Protana Colegory, 19\%

(Million Kilowathours) (Continned)

\begin{tabular}{|c|c|c|c|}
\hline 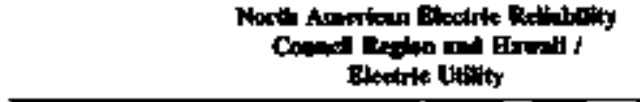 & Ensters & 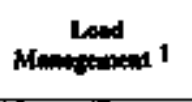 & $\begin{array}{l}\text { Ted DGM } \\
\text { Propans }\end{array}$ \\
\hline
\end{tabular}

mocctus) (Cwatimed)

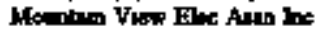

Numopact Eloctic Coop Int

Nowin Fower $C 0$

Penfic Gan \& Elector Co

Puatcorp

Palo Aho Cing of

Pationen Oay of

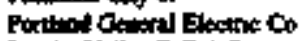

Pondive Villoy R B A we

Puble Sermot Co of Coldonto

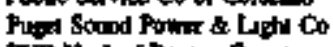

Pud vo 1 of Bento. Condy

PUD Ro I af C7, 100 .

ftud ito 1 of hod Darle Cay

PUd Wo 2 of Ore Conty

Relinge City of

Raverile Giy of

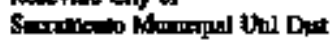

Snlew Eloxtac Coop

Sul Riva Proy Ag 1 \& P Den

So Dato Cos \& Elatinc Co

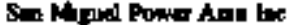

Sell Con City of

Sarill Oxy of

Souther Cohfonn Bton Co

Sprofict Coy of

stopur Senpe vatey B C he

Troonen Chy of

Tros Fintere Coop les

Tweoson Eletene Power Oo

Triloxk binjerom Duder

trand Polne bic

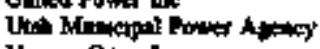

Venos ofy of

Wathengion Water Pomer Co

Yalownoes Vally Ele Ca-op

wrocc(us) Twat

Coolinene ES.

Aftec

Alute Eextre Lytheapones Co

Golda Volvo Eloc Ane In

ASCC Totel

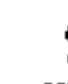

โ51

3.0.1

1.011

12

25

738

332

1.835

4

91

9I

6

648

94

0
545

525
6185

6.1\%

0

410

96

15

5

508
0

$2+277$

59,20;5;

2

3,021

1,237

12

23

I

ist

1,835

4
12

8

27

6

648

2

149

$+$

525

6.185

80

410

96

is

$-2$

3

500

1969

1,76

E:arid

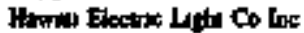

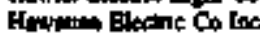

Mind Elonitio Co Lod

Anirtis Tut

Us. Total

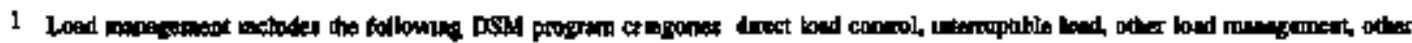

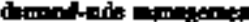

- Vala lase inos 0 .

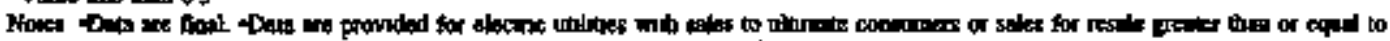

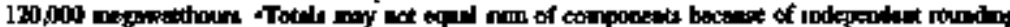

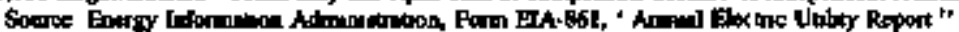


Table 11. U.S. Electric Utiky Enexsy Stuveng by North American Electric Relinbility Conneil Region and Hawiii by Class of Owershlp and Sector, 1996 (Million Kilowatthours)

\begin{tabular}{|c|c|c|c|c|c|c|}
\hline 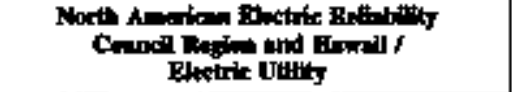 & Clan of & 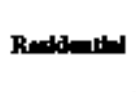 & Compird & Imopirtal & Dibar & Total \\
\hline 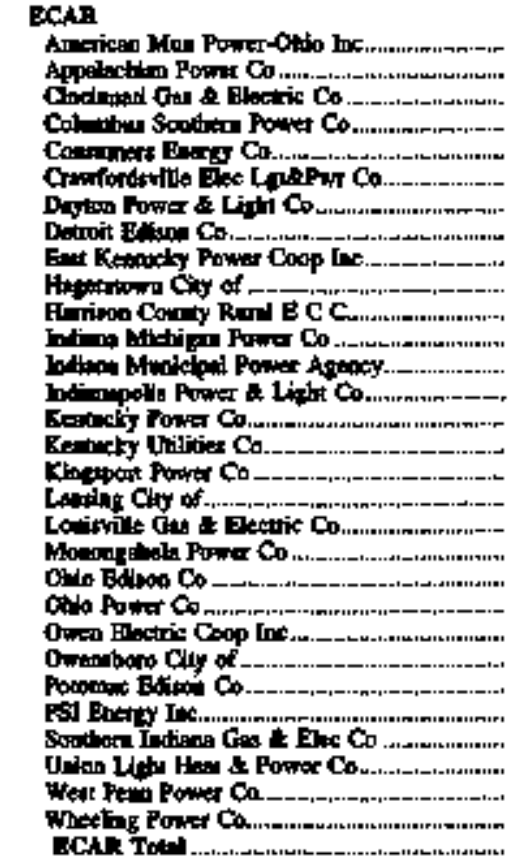 & 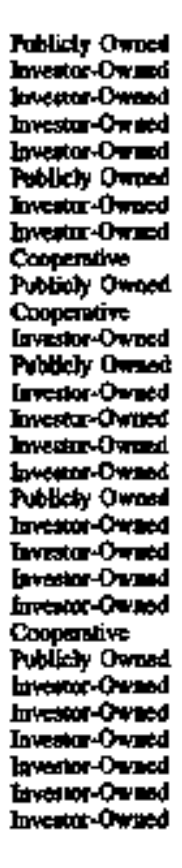 & $\begin{array}{r}0 \\
89 \\
3 \\
58 \\
74 \\
19 \\
23 \\
3 \\
* \\
0 \\
24 \\
7 \\
19 \\
27 \\
46 \\
9 \\
0 \\
1 \\
74 \\
102 \\
49 \\
1 \\
0 \\
192 \\
96 \\
5 \\
0 \\
36 \\
2 \\
1087\end{array}$ & $\begin{array}{r}0 \\
5 \\
429 \\
4 \\
95 \\
0 \\
92 \\
63 \\
0 \\
0 \\
0 \\
6 \\
0 \\
55 \\
1 \\
1 \\
0 \\
4 \\
4 \\
00 \\
58 \\
3 \\
4 \\
0 \\
142 \\
229 \\
29 \\
1 \\
89 \\
0 \\
1406\end{array}$ & 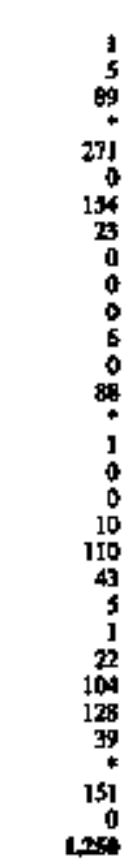 & $\begin{array}{l}0 \\
0 \\
0 \\
0 \\
0 \\
0 \\
0 \\
0 \\
0 \\
0 \\
0 \\
0 \\
0 \\
0 \\
0 \\
0 \\
0 \\
0 \\
0 \\
0 \\
0 \\
0 \\
0 \\
0 \\
0 \\
3 \\
0 \\
0 \\
0 \\
0 \\
3\end{array}$ & $\begin{array}{r}1 \\
99 \\
522 \\
53 \\
441 \\
365 \\
109 \\
3 \\
0 \\
35 \\
161 \\
28 \\
48 \\
9 \\
14 \\
254 \\
203 \\
57 \\
2 \\
22 \\
439 \\
456 \\
76 \\
1 \\
276 \\
2 \\
365\end{array}$ \\
\hline 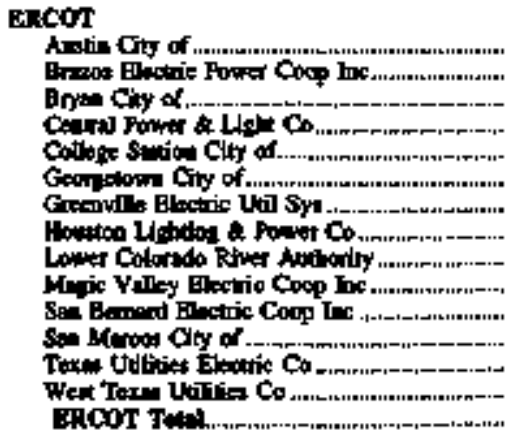 & 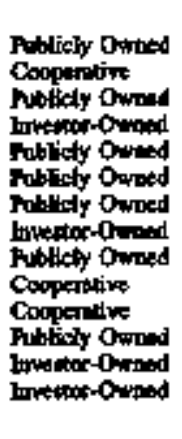 & 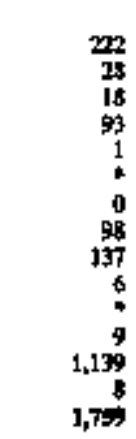 & $\begin{array}{r}34 \\
* \\
40 \\
2 \\
0 \\
0 \\
121 \\
23 \\
0 \\
0 \\
2 \\
1321 \\
10 \\
2414\end{array}$ & $\begin{array}{c}0 \\
0 \\
0 \\
0 \\
0 \\
0 \\
13 \\
0 \\
0 \\
0 \\
0 \\
0 \\
30 \\
0\end{array}$ & $\begin{array}{l}0 \\
0 \\
0 \\
0 \\
0 \\
0 \\
0 \\
0 \\
0 \\
0 \\
0 \\
0 \\
0 \\
0 \\
0\end{array}$ & $\begin{array}{r}546 \\
29 \\
18 \\
134 \\
2 \\
4 \\
247 \\
160 \\
6 \\
11 \\
2,660 \\
68 \\
3,46\end{array}$ \\
\hline 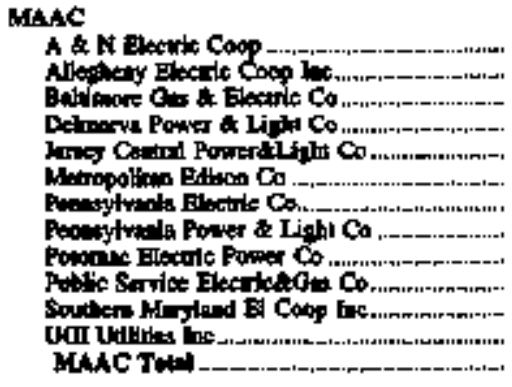 & 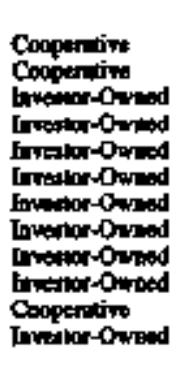 & $\begin{array}{l}2 \\
35 \\
40 \\
78 \\
79 \\
33 \\
79 \\
172 \\
97 \\
15\end{array}$ & $\begin{array}{r}0 \\
0 \\
489 \\
91 \\
167 \\
6 \\
28 \\
5 \\
1,402 \\
570 \\
0 \\
0 \\
2,756\end{array}$ & $\begin{array}{r}0 \\
0 \\
0 \\
0 \\
0 \\
8 \\
40 \\
1 \\
0 \\
102 \\
0 \\
0 \\
2 \\
21\end{array}$ & $\begin{array}{l}0 \\
0 \\
0 \\
0 \\
0 \\
0 \\
0 \\
0 \\
0 \\
0 \\
0 \\
0 \\
1\end{array}$ & $\begin{array}{r}2 \\
525 \\
131 \\
244 \\
93 \\
108 \\
995 \\
1591 \\
59 \\
509\end{array}$ \\
\hline 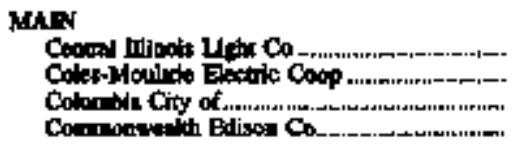 & 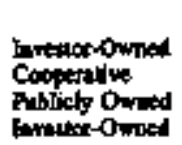 & $\begin{array}{l}1 \\
0 \\
6 \\
0\end{array}$ & $\begin{array}{r}0 \\
4 \\
3 \\
25\end{array}$ & 0 & $\begin{array}{l}0 \\
0 \\
0 \\
0\end{array}$ & $\begin{array}{r}1 \\
9 \\
25\end{array}$ \\
\hline
\end{tabular}

See footodica ct eod of thle. 
Table 11. U.S. Electric Utility Energy Saviazs by North Anerican Flectric Reliah"lity Councti Region and Hamell by Class of Omership and Sector, 1996 (Million Kilowatthouss) (Continued)

\begin{tabular}{|c|c|c|c|c|c|c|}
\hline 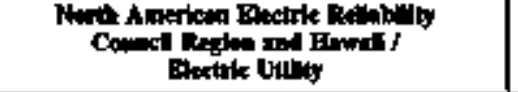 & Oax of & Reold. - Itl-1 & Conerelal & 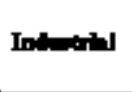 & Othar & Tatel \\
\hline 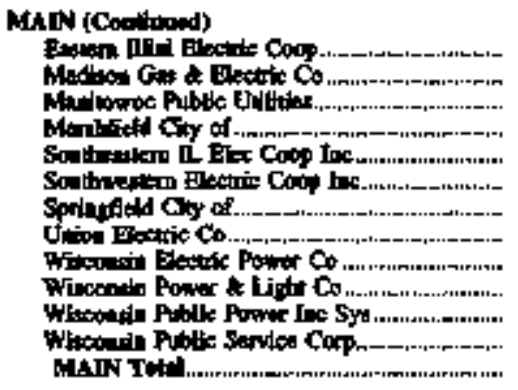 & 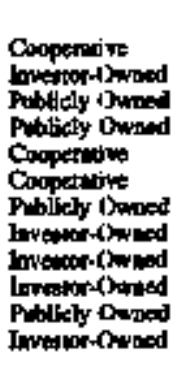 & $\begin{array}{r}3 \\
30 \\
4 \\
7 \\
4 \\
4 \\
525 \\
40 \\
129 \\
357\end{array}$ & $\begin{array}{r}0 \\
140 \\
5 \\
4 \\
0 \\
11 \\
0 \\
511 \\
349 \\
12 \\
382 \\
1,741\end{array}$ & $\begin{array}{r}0 \\
5 \\
1 \\
0 \\
0 \\
5 \\
401 \\
0 \\
40 \\
40\end{array}$ & $\begin{array}{r}0 \\
20 \\
0 \\
0 \\
0 \\
0 \\
0 \\
0 \\
21 \\
0 \\
30\end{array}$ & $\begin{array}{r}193 \\
14 \\
5 \\
1 \\
15 \\
5 \\
1,757 \\
417 \\
36 \\
546 \\
3 \% 4\end{array}$ \\
\hline 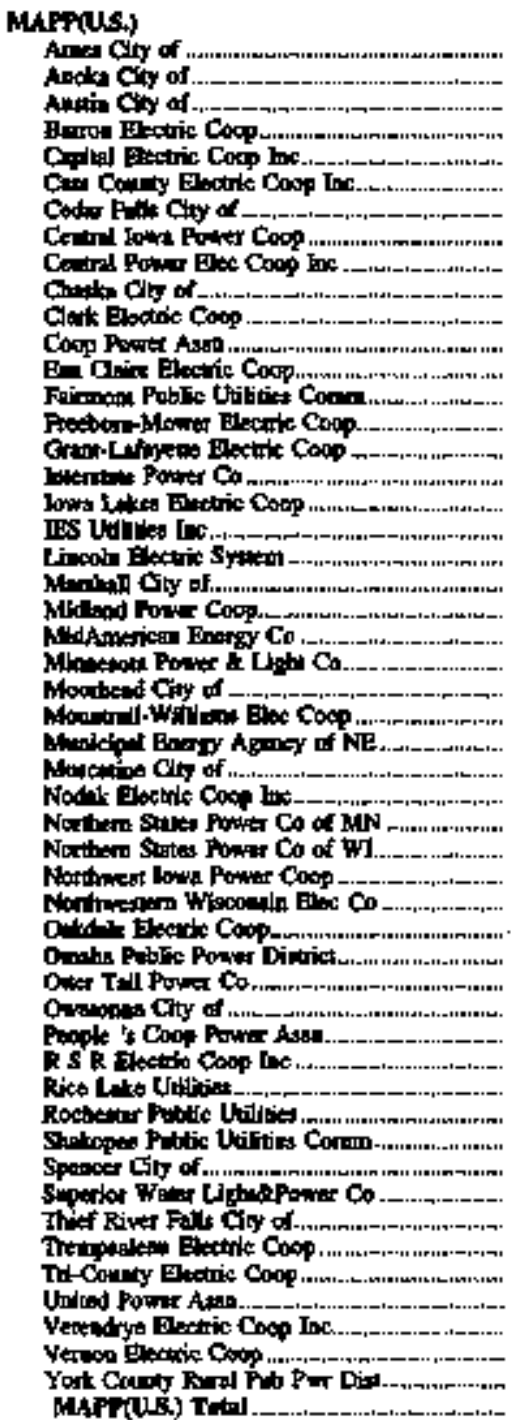 & 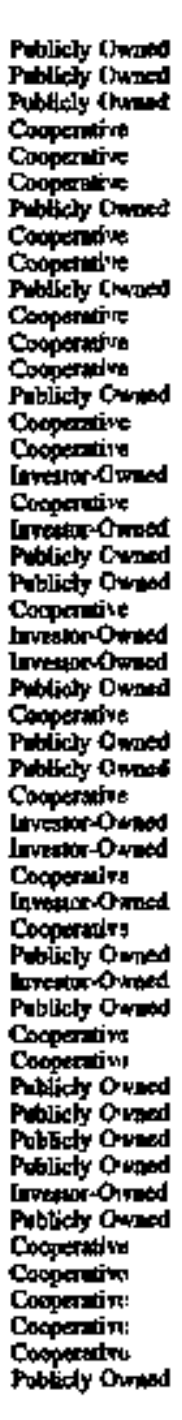 & 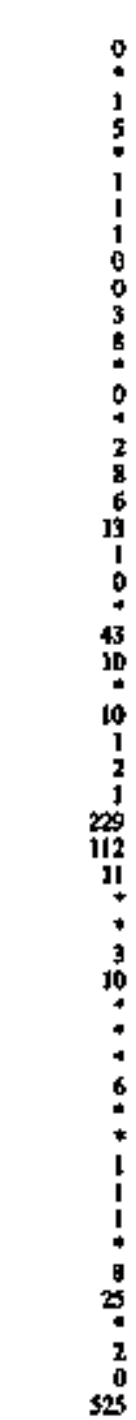 & 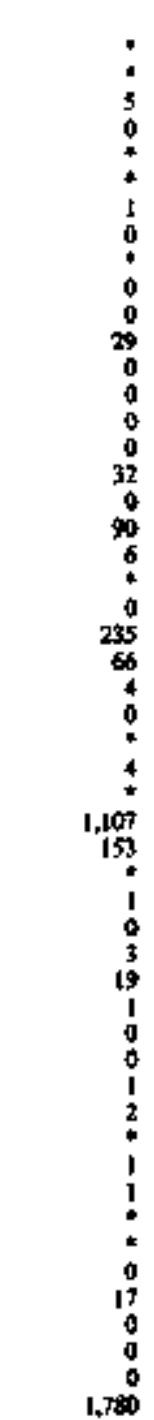 & 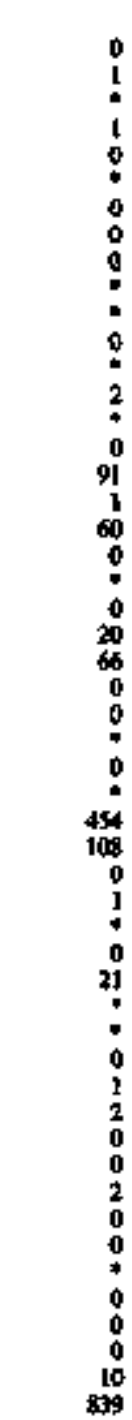 & $\begin{array}{l}1 \\
0 \\
0 \\
0 \\
0 \\
0 \\
0 \\
0 \\
0 \\
0 \\
0 \\
0 \\
0 \\
0 \\
0 \\
0 \\
0 \\
0 \\
0 \\
0 \\
0 \\
0 \\
0 \\
0 \\
0 \\
0 \\
0 \\
0 \\
0 \\
0 \\
0 \\
0 \\
0 \\
0 \\
0 \\
0 \\
0 \\
0 \\
0 \\
0 \\
0 \\
0 \\
0 \\
0 \\
0 \\
0 \\
0 \\
0 \\
0\end{array}$ & 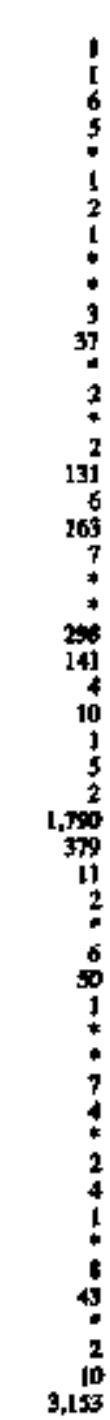 \\
\hline
\end{tabular}

Sec foctenotes at end af thble. 
Table 11. U.S. Electric Uthity Energ Savings by North Ameriean Deetric Reliability Cauncil Region and Hawali by Class of Ownertint and Sector, 19\%6 (Million Kilowatthours) (Continued)

\begin{tabular}{|c|c|c|c|c|c|c|}
\hline 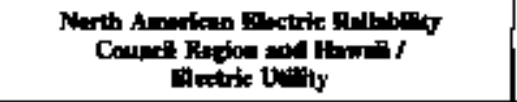 & ond of & Rowile thal & Conomentil & 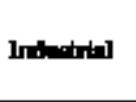 & Oher & Totid \\
\hline 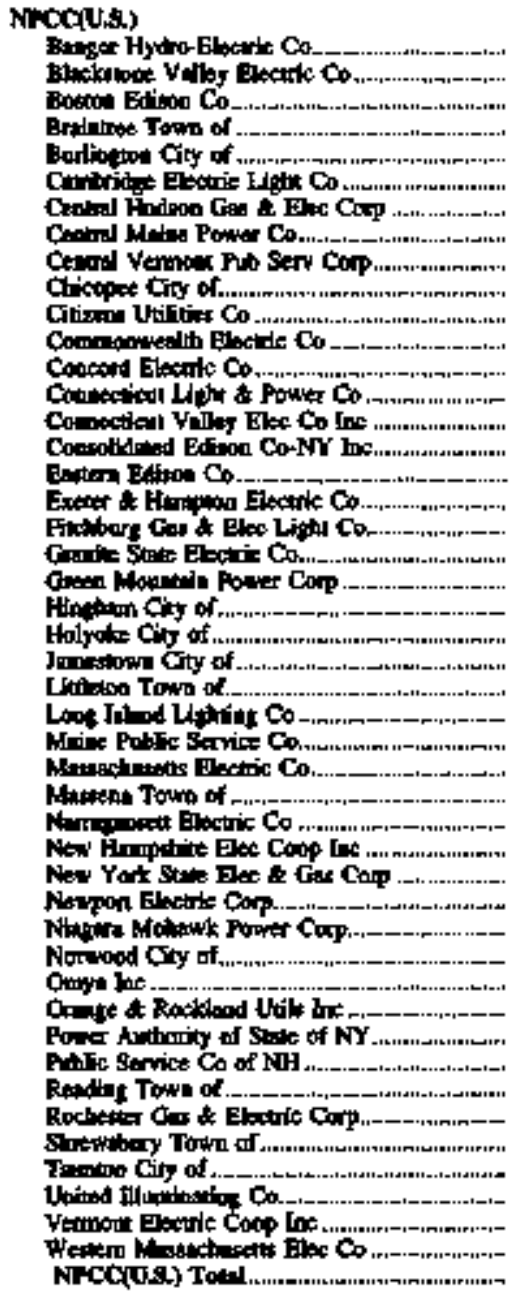 & 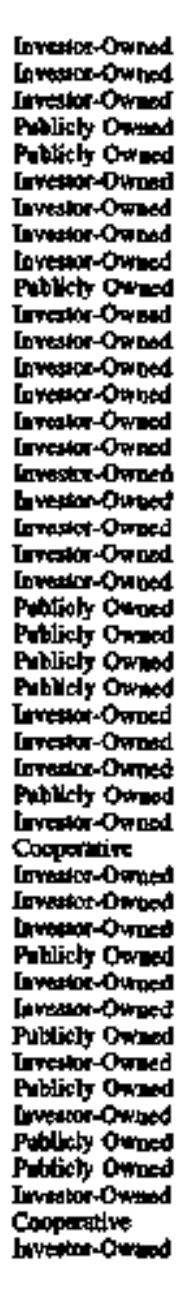 & $\begin{array}{r}34 \\
4 \\
0 \\
18 \\
11 \\
114 \\
30 \\
10 \\
13 \\
2 \\
304 \\
1 \\
200 \\
31 \\
3 \\
1 \\
6 \\
10 \\
3 \\
7 \\
0 \\
4 \\
157 \\
3 \\
142 \\
0 \\
25 \\
4 \\
166 \\
9 \\
276 \\
1 \\
4 \\
81 \\
40 \\
8 \\
4 \\
1 \\
1 \\
70 \\
70 \\
75 \\
1893\end{array}$ & 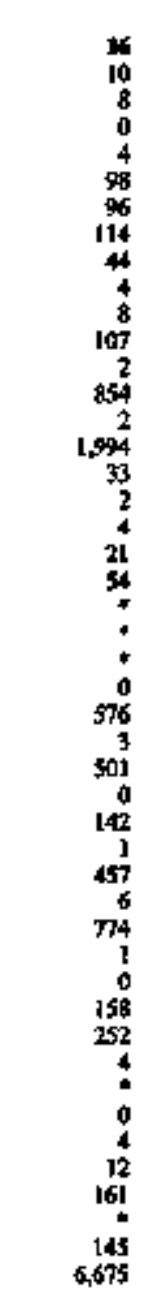 & $\begin{array}{r}4 \\
21 \\
2 \\
15 \\
15 \\
0 \\
36 \\
235 \\
19 \\
1 \\
7 \\
0 \\
3 \\
181 \\
1 \\
0 \\
13 \\
2 \\
8 \\
13 \\
0 \\
0 \\
5 \\
0 \\
0 \\
0 \\
0 \\
307 \\
0 \\
87 \\
0 \\
0 \\
2 \\
102 \\
3 \\
0 \\
0 \\
7 \\
8 \\
0 \\
202 \\
0 \\
0 \\
41 \\
0 \\
15 \\
145\end{array}$ & $\begin{array}{l}0 \\
0 \\
0 \\
0 \\
0 \\
0 \\
0 \\
1 \\
0 \\
0 \\
0 \\
0 \\
0 \\
0 \\
0 \\
0 \\
0 \\
0 \\
0 \\
0 \\
0 \\
0 \\
3 \\
0 \\
0 \\
0 \\
2 \\
0 \\
1 \\
0 \\
0 \\
0 \\
0 \\
0 \\
0 \\
0 \\
0 \\
0 \\
0 \\
0 \\
0 \\
0 \\
0 \\
2 \\
5 \\
5 \\
20\end{array}$ & 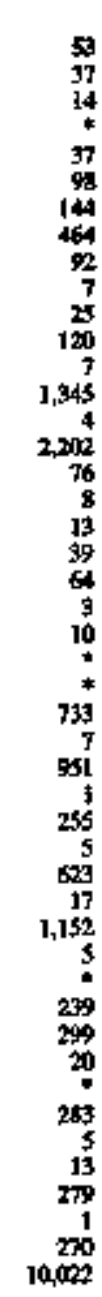 \\
\hline 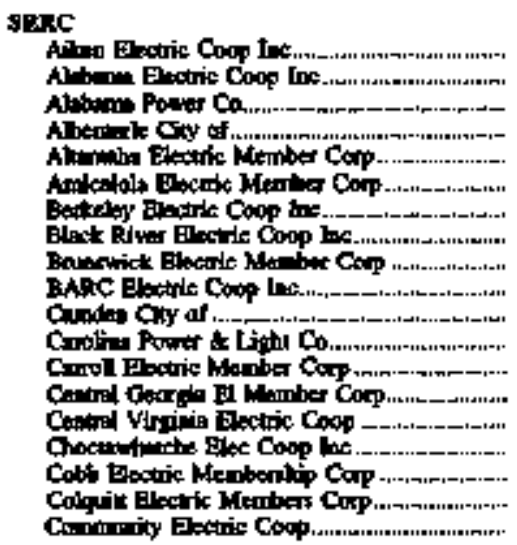 & 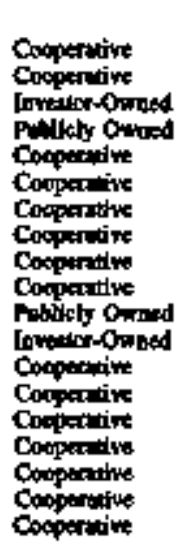 & $\begin{array}{r}2 \\
43 \\
-589 \\
0 \\
7 \\
7 \\
0 \\
0 \\
790 \\
* \\
4 \\
0 \\
6 \\
2 \\
* \\
0\end{array}$ & $\begin{array}{r}: \\
0 \\
27 \\
0 \\
0 \\
0 \\
0 \\
0 \\
0 \\
369 \\
0 \\
0 \\
0 \\
0 \\
0\end{array}$ & $\begin{array}{r}0 \\
0 \\
0 \\
0 \\
0 \\
0 \\
0 \\
0 \\
0 \\
0 \\
885 \\
0 \\
0 \\
0 \\
0 \\
0 \\
0 \\
0\end{array}$ & $\begin{array}{l}0 \\
0 \\
0 \\
0 \\
0 \\
0 \\
0 \\
0 \\
0 \\
0 \\
0 \\
0 \\
0 \\
0 \\
0 \\
0 \\
0 \\
0 \\
0\end{array}$ & $\begin{array}{r}2 \\
43 \\
-562 \\
\vdots \\
\vdots \\
7 \\
\vdots \\
0 \\
2,044 \\
4 \\
1 \\
6 \\
2 \\
7 \\
0\end{array}$ \\
\hline
\end{tabular}

Sex footoris at od of the 
Table 11. U.S. Electric Utllty Energy Savinos by North American Electric Reliability Counci Region and Hawati by Cless of Owhership and Sector, 1996 (Million Kilowathours) (Contuned)

\begin{tabular}{|c|c|c|c|c|c|c|}
\hline 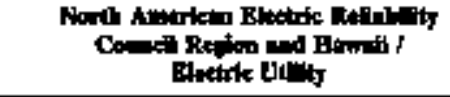 & $\begin{array}{c}\text { Qhy of } \\
\text { Owberstp }\end{array}$ & 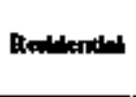 & Cosmondal & latelat & Other & Twath \\
\hline 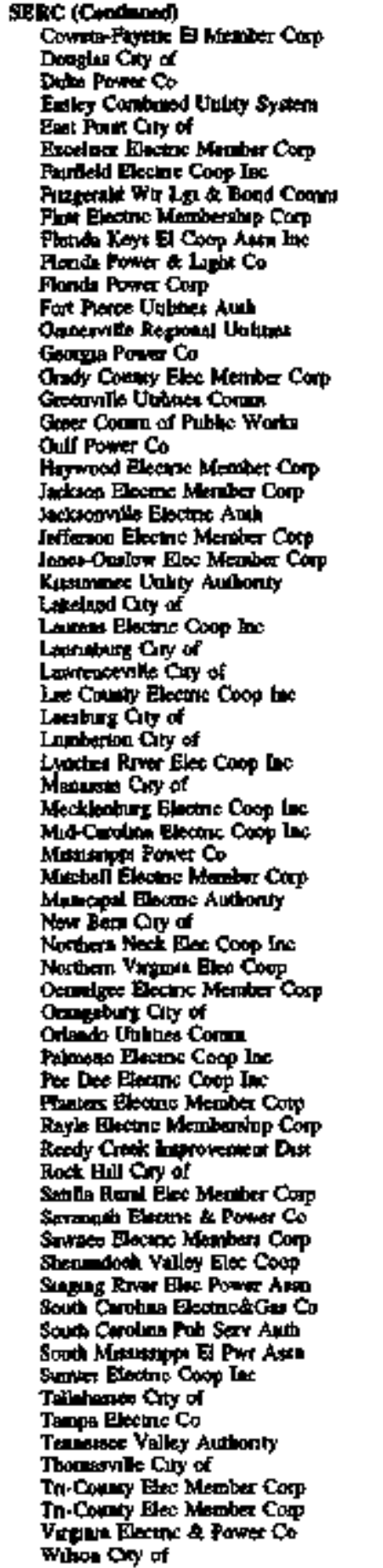 & 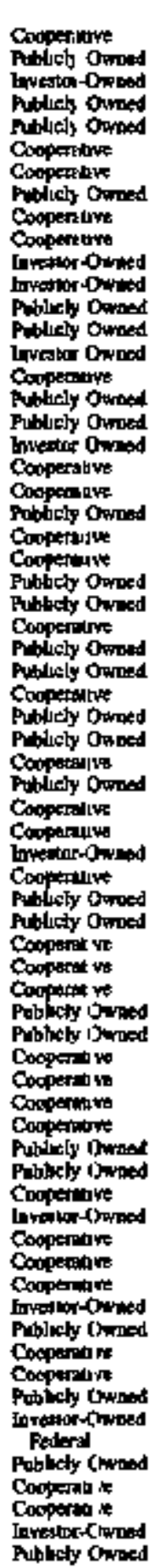 & 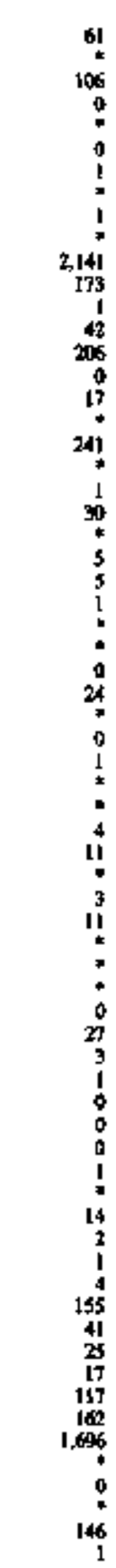 & 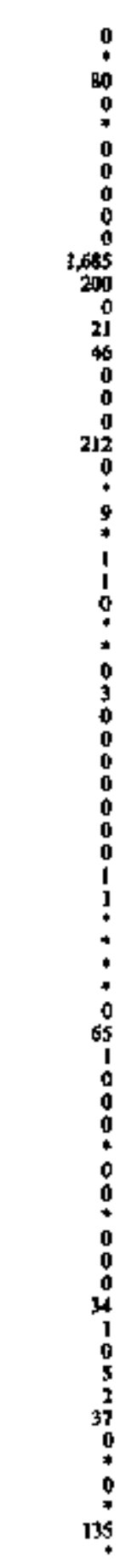 & 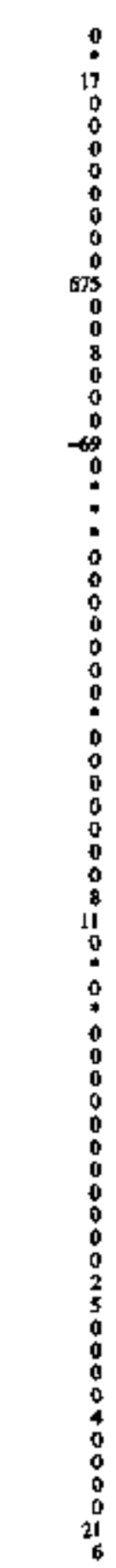 & 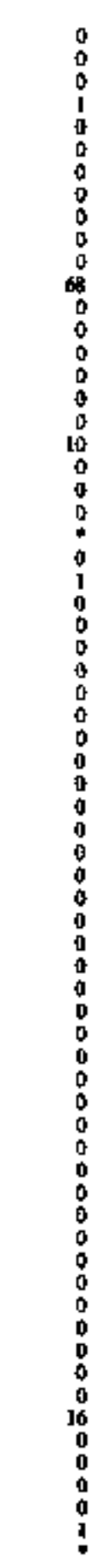 & 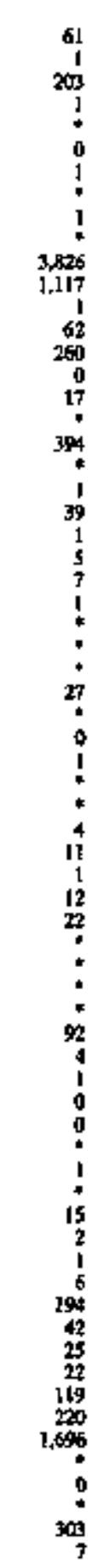 \\
\hline
\end{tabular}

Sect foothont at end of rable 
Table 11. U.S. Wectric Utilly Energ Savings by North American Electric Reliebitity Connell Region and Hawati by Class of Owuership and Sector, 1996

(Million Kilowathour\$) (Continued)

\begin{tabular}{|c|c|c|c|c|c|c|}
\hline 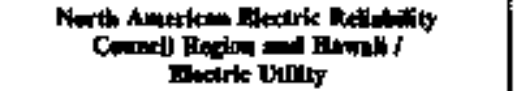 & Otive tof & Restenalin & Comnerrdel & Inimatili & Detitr & Total \\
\hline 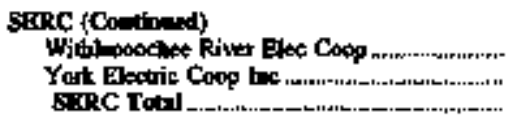 & $\begin{array}{l}\text { Copphative } \\
\text { Corparive }\end{array}$ & 5.794 & $\begin{array}{r}0 \\
1438\end{array}$ & $\begin{array}{r}0 \\
1,574\end{array}$ & $\begin{array}{l}0 \\
0 \\
97\end{array}$ & 10.404 \\
\hline 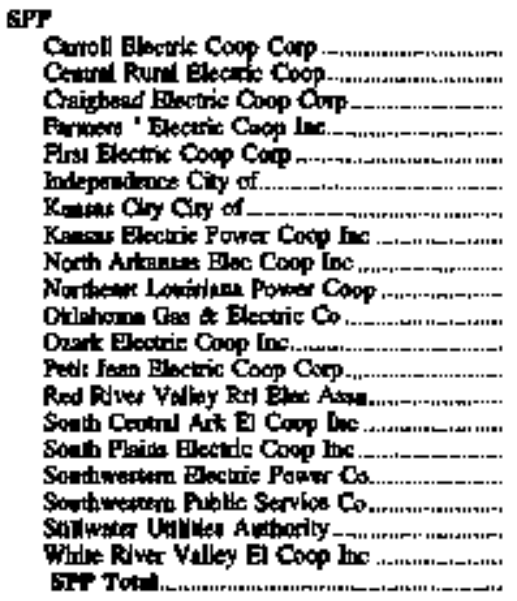 & 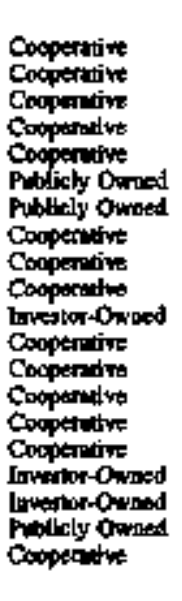 & $\begin{array}{r}0 \\
3 \\
0 \\
0 \\
6 \\
3 \\
* \\
0 \\
0 \\
0 \\
12 \mathbf{1} \\
6 \\
\mathbf{0} \\
2 \\
0 \\
7 \\
\mathbf{4} \\
1 \mathbf{2 6} \\
\mathbf{0} \\
\mathbf{0} \\
\mathbf{3} \mathbf{4}\end{array}$ & $\begin{array}{r}0 \\
0 \\
0 \\
0 \\
0 \\
0 \\
1 \\
1 \\
0 \\
10 \\
0 \\
0 \\
0 \\
0 \\
0 \\
0 \\
0 \\
0 \\
0 \\
12\end{array}$ & $\begin{array}{r}0 \\
0 \\
+ \\
* \\
0 \\
0 \\
0 \\
0 \\
0 \\
0 \\
0 \\
0 \\
1 \\
3 \\
0 \\
0 \\
9 \\
0 \\
0 \\
14\end{array}$ & $\begin{array}{l}0 \\
0 \\
0 \\
0 \\
0 \\
0 \\
0 \\
1 \\
0 \\
0 \\
0 \\
0 \\
0 \\
0 \\
0 \\
1 \\
0 \\
6 \\
0 \\
0 \\
8\end{array}$ & 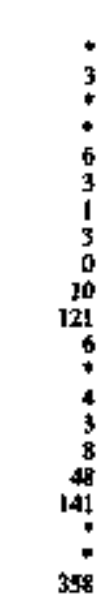 \\
\hline 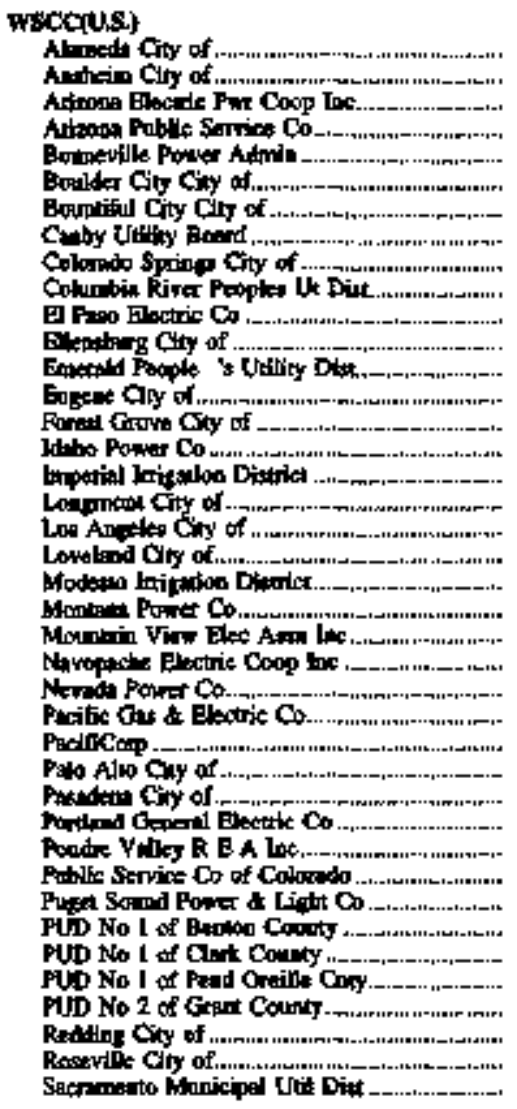 & 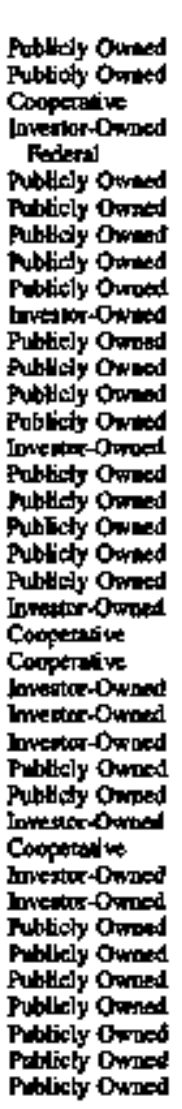 & 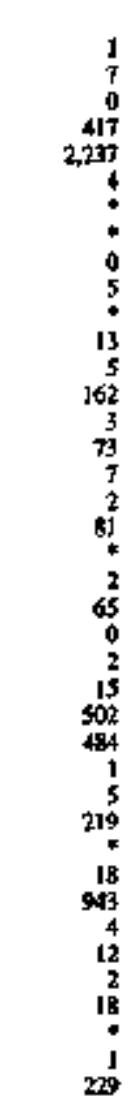 & 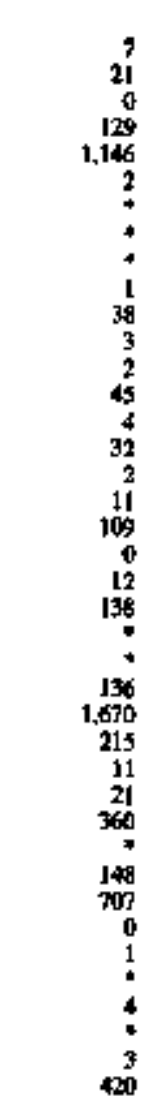 & $\begin{array}{r}9 \\
0 \\
0 \\
73 \\
0 \\
0 \\
4 \\
0 \\
0 \\
0 \\
5 \\
23 \\
1 \\
51 \\
0 \\
2 \\
50 \\
0 \\
0 \\
27 \\
0 \\
5 \\
0 \\
523 \\
558 \\
0 \\
0 \\
159 \\
0 \\
167 \\
156 \\
0 \\
0 \\
154 \\
0 \\
3 \\
0\end{array}$ & $\begin{array}{r}0 \\
0 \\
0 \\
0 \\
642 \\
0 \\
0 \\
0 \\
0 \\
0 \\
0 \\
0 \\
0 \\
1 \\
0 \\
28 \\
0 \\
0 \\
32 \\
3 \\
30 \\
20 \\
0 \\
0 \\
0 \\
0 \\
325 \\
0 \\
0 \\
0 \\
0 \\
0 \\
0 \\
0 \\
0 \\
0 \\
0 \\
0 \\
0 \\
0 \\
0 \\
0 \\
0\end{array}$ & 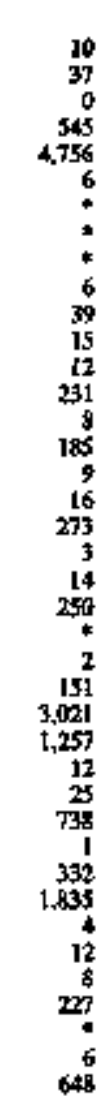 \\
\hline
\end{tabular}

Sed footooket ti cod of tuble 
Table 11. U.S. Electric Utility Rnergy Savings by North Aroerican Electric Rellability Counct Region and Howail by Class ol Ownership and Sector, 1996

(Million Kilowathoors) (Continued)

\begin{tabular}{|c|c|c|c|c|c|c|}
\hline 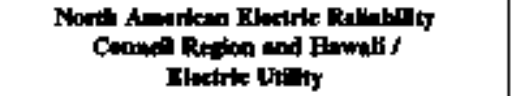 & 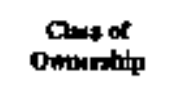 & Re:lentillal & Cenneridal & 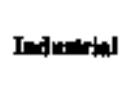 & Ontwy & $T$ Tat \\
\hline 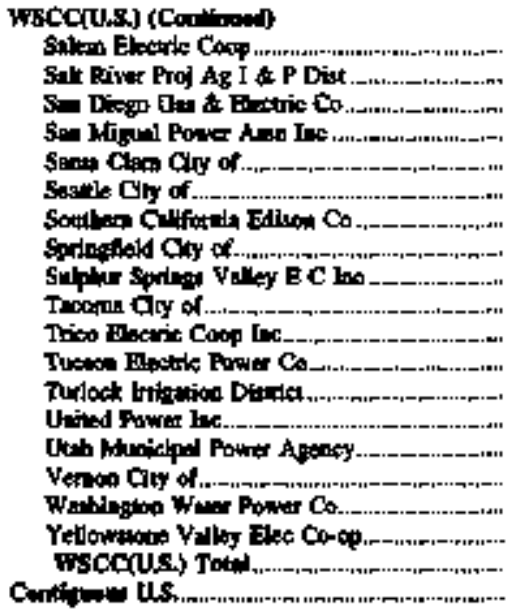 & 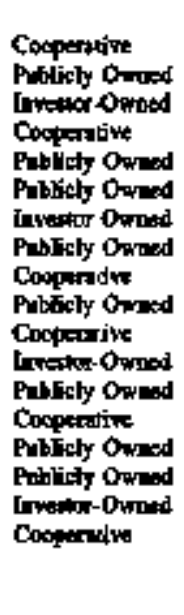 & $\begin{array}{r}2 \\
85 \\
169 \\
0 \\
0 \\
192 \\
1,2106 \\
58 \\
0 \\
122 \\
0 \\
14 \\
7 \\
-2 \\
4 \\
0 \\
449 \\
9 \\
7,840 \\
2455\end{array}$ & $\begin{array}{r}0 \\
63 \\
821 \\
0 \\
0 \\
264 \\
3,004 \\
14 \\
0 \\
144 \\
0 \\
81 \\
3 \\
0 \\
2 \\
0 \\
42 \\
0 \\
9,835 \\
2,164\end{array}$ & $\begin{array}{r}0 \\
0 \\
0 \\
0 \\
4 \\
43 \\
1,762 \\
9 \\
0 \\
144 \\
0 \\
0 \\
5 \\
0 \\
0 \\
3 \\
21 \\
0 \\
4633 \\
14,409\end{array}$ & $\begin{array}{r}0 \\
0 \\
0 \\
0 \\
0 \\
x \\
193 \\
0 \\
0 \\
0 \\
0 \\
0 \\
0 \\
3 \\
0 \\
0 \\
0 \\
1,356 \\
1,530\end{array}$ & $\begin{array}{r}1 \\
149 \\
981 \\
505 \\
6.185 \\
80 \\
4 \\
410 \\
4 \\
96 \\
15 \\
-2 \\
5 \\
3 \\
508 \\
9 \\
23.653 \\
61,75\end{array}$ \\
\hline 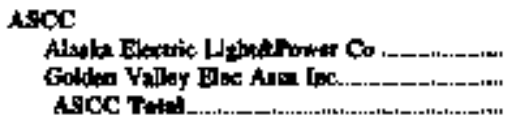 & 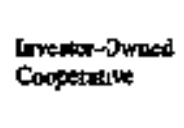 & $\begin{array}{l}\mathbf{0} \\
\mathbf{3} \\
\mathbf{3}\end{array}$ & ! & 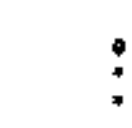 & $\begin{array}{l}6 \\
0 \\
0\end{array}$ & $\begin{array}{l}4 \\
5 \\
5\end{array}$ \\
\hline 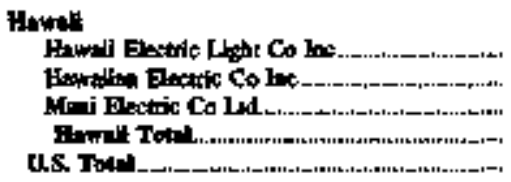 & 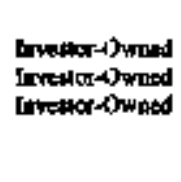 & $\begin{array}{r}5 \\
5 \\
5\end{array}$ & $\begin{array}{r}3 \\
11 \\
4 \\
19 \\
29,14\end{array}$ & 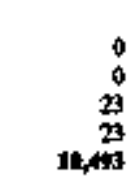 & $\begin{array}{r}0 \\
0 \\
0 \\
1,51\end{array}$ & $\begin{array}{r}9 \\
12 \\
28 \\
49 \\
61,20\end{array}$ \\
\hline
\end{tabular}

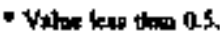

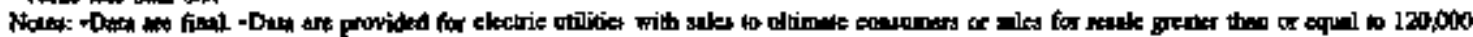

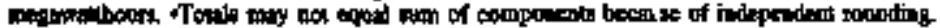

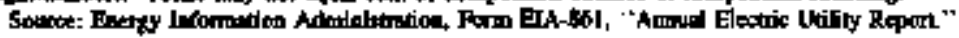




\section{Peak Load Reductions}

One of the main goals of DSM prograns is to reduce a utilily's peak load through energy efficiency and load control prograns. Peak load reductions (measured in megawatts (MW)) are eategorized as potential or actial. Potential peak load reductions are the anlount of load avajlable for curtailment through load control programs such as direct load control, interruptible joad eontrol, other load managentent, or other DSM programs. Actual peak load reductions are the amount of reduction that is achieved from load control programs that are put into force at the same time as peak joad and the amount of rednetions that result from energy efficiency programs at the time of peak load.

Utilities are required to report both potential and actual peak load reductions on Form EIA-861 for the direct load control, interruptible load control, othes Ioad mantagement, apd other DSM prograns categories. Utilities are only required to teport actual peak load reductious from energy efficiency programs. Actual and potential peak load reductions are generally the same for energy efficiency programs. These programs are focused on reducing energy consumption and operate over miany hours during the year and not specifically during the time of peak load. However, to allow for mort accurate comparisons and data anaEyses to be conducted, in this publication it is assumed that potential peak load redactions resulting from energy efficiency programs were equal to actual peak load reductions. Only large utilities are requited to report annual effects for actual and potential peak load teductions; small utilities teport only incremental peak load reductions. ${ }^{\mathrm{B}}$

\section{Annual Effocts for Actual Poak Load Reductions}

In 1996, actual peak load reductions were 29,893 MW. Actual peak load reductions ate predicted by utilities to increase to $32,361 \mathrm{MW}$ in 1997 and to $36,892 \mathrm{MW}$ in 2001 (Table 12).

For the 1996 reporting year, investor-owned ubilities accounted for 73.9 percent of actual peak load reductions. Publicly owbed utilities accounted for 9.2 percent, followed by cooperatives with 9.2 percent, and Federally owned with 7.8 percent. Utility forecasts indicated that investor-owned utilities are expected to increase acoul pear Joad reductions by 11.7 percent in 1997 and to increaste at an annual rate of 3.2 percent through 2001. In $200 \mathrm{t}$, cooperatives are expected to provide 8.6 percent of actual peak load reductions and publicly owned utilities are expected to provide 7.9 percent (Table 12). ${ }^{\$}$ Cooperatives have the greatest peak load reductions as a percentage of utility peak load because, as purchasers of wholesale power, which is more expensive during peak periods, they focus on peak load reductions rather than energy savings. For this reason, it is economically efficient for cooperatives to reduce their system peak load as much as possible (Figure 5).

The 100 otilities with the greatest actual peak load reductions in [ 996 ascounted for $\mathbf{8 7 . 8}$ percent of the total peak load reduction. The 50 utilities with the greatesi peak load reduetions accounted for 76.3 percent of the total, and the top 25 utilities accounted for 62.1 percent (Figure 6). These 100, 50, and 25 utilities with the greatest actual peak load reductions represented 53.4, 38.0, and 26.5 percent, respectively, of total retail sales of eloctricity in the United States in 1996 .

Energy efficiency programs accounted for the greatest share of actual peak load reductions, 47.6 percent of the $29,893 \mathrm{MW}$ of total actuad peak load teductions. Interruptible load, primasily an industrial sector program, contributed 24.7 percent of the total (Figure 7). Direct load control programs accounted for 18.6 percent of actual peak load reductions. Other load management and other DSM programs combined for the remaining 9.0 percent of total peak load reductions (Table 13). Interruptible loed control programs decreased 12 percent from 1995 to 1996. The actual peak load reductions that are predicted for 1997 and 2001 indicate increases in afl categories except other DSM and other load masagement where a decrease is predicted for 1997 . The greatest increase from 1996 to 1997 is predicted for the interruptible load program category, an increase of $1,456 \mathrm{MW}$. The greatest percentage of increase from 1996 to 1997 , 19.7 percent, is expected from the interruptible program category. From 1997 to 2001, the sverage annual increase for ac1ual peak loat reductions is expected to be approximately 3.3 percent, with the greatest average annual growth rate predjcted for direct load control programs at 4.9 percent (Tables 13 and 18).

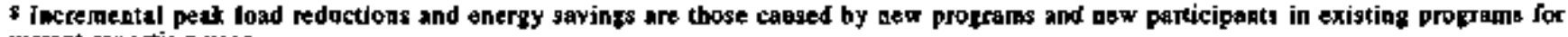
the current reporting yeor.

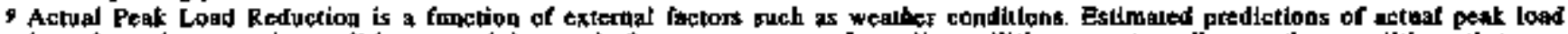
redoctions deperd on certsin conditions remaiaing adatic from year te year. In reality, utilities chatot predict westher conditions lbal may aflect dafa for the forectast period.
} 
In 5996 , the residential sector accourited for 38.4 percent of actual peak load reductions; the commercial sector, 29.0 percent; the industrial sector, 30.4 percent; and the "other" sector, 2.2 percent. The residential sector's share was grealest primarily because of the volume of participants in entrgy tificiency and direct load control programs. The greatest percentoge of increase in axtual peak load reductions from 1995 to 1996 was in the "other" sector with 21.3 percent. The residential sector increased actual peak load seductions 4.9 perceat and the commercial sector increased 7.7 percent, while the industrial sector decreased by 9.5 percent (Tables 14 and 2.0 ).

The NERC togion with the greatest actual peak load reductions in 1996 was SERC with 34.1 percent of total U.S. peak load reductions, partly besause several large utilities that bad the largest load management programs in the United States are included. The WSCC region had the second greatest peak load reductions, contributing 17.2 percent of the total peak load reductions for 1996 . The greatest increase in peak load reductions at $368 \mathrm{MW}$ occumed in the MAIN region, which also had the greatest percentage of increase at 29.3 percent. For 1997, the MAAC region is predicted to increase another $4 \mathbf{L}$.3 percent. From 1997 to 2001, the MAIN region is predicted to increase at an annual rate of 10.5 percent íTable 18).

\section{Potential Peak Load Reductions}

In 1996, potential poak load reductions increased 2.9 percent to 48,344 MW. For 1997, potential reductions are predicted to ipcrease 3.4 percent to $49,993 \mathrm{MW}$ and to $\$ 4,968 \mathrm{MW}$ by $200 \mathrm{l}$.

Is 1996, investor-owned utilities accounied for 72.5 percent of the total potential peat load reduction; cooperative utilities accounted for 10.8 percent; Federally owned. 9.2 percent; and publicly owned, 7.5 percent. The greatest percentage of increase, 10.9 percent. was reported by publicly owned electric utilities. Fot 2001, publicly owned utilitios are predicted to have the greatest annual rate of increase, 4,3 percent. Investor-owned otilities are predisted to continue to account for the greatest share of potential peak load reductions in 2001 at 72.2 percent.

Interruptible load programs accounted for 44.6 percent of potential peak load reductions in I996; energy efficiency accounted for 29.5 perient; direct load control for 19.5 percent; and other load management and other DSM programs, combioed, accounted for 6.4 percent. When comparing historical potential peak load reductions to projected potential peak load reductions, other DSM programs for 1996 and 1997 aceonnted for the greatest percentage increase. For 2001, the greatest average antual increase, 4.] percent, is predicted for energy efficjency programs. In 200T, the greatest share of potential peal load reduction is expected for interruptible toad programs (Table 13).

The industrial sector acconnted for 41.9 percent in 1996, the greatest share of potential peak load reductions, primarily as a result of interruptible load progtams. The residential and commercial sectors contributed 30.4 percent and 25.8 percent, respectively, in 1996 . The other sector accounted for 1.9 percent

In 1996, the SERC region accounted for 33.7 percent of the total poteatial peak load reductions, primarily because the Tenuessee valley Authority, Carolisa Power and Light, Duke Power, Florida Power and Light, and Florida Power Corporation are included. The SERC region is forecasted to continue to contribute the greatest share of potential perak reductions in 1997 and 2001 .

\section{Incremental Effects for Actual Peak Load Reduction}

In 1996, large otilities reported incremental actual peak load reductions of $3,689 \mathrm{MW}$. All of the ownership classes reported a decrease ovet 1995 levels except for Federal utilities. Investor-owned electric utilities continued to acconnt for the grealest share of incremental reductions, $\mathbf{8 4 . 4}$ percent (Tabje 15).

A]l DSM ptogram calegories reported decreases in jncremental actual peak load reductions for large utilitjes in 1996. Interruptible load control programs accounted for the Jargest pereentage of incremeatal actual peak load reductions.

For large utilities, the industrial sector accounted for the greatest percent of actual peak load reductions, 50.7 percent. For small utilities, the residential sector accounted for the greatest amount, 57.7 percent, of actual peak load reductions (Table 17). 
Table 12. U.S. Electric Utllity Actual and Potential Peak Loud Reductions by Class of Owisership, 1992 Through 1996, 1997, and 2001

(Megawalts)

\begin{tabular}{|c|c|c|c|c|c|c|c|}
\hline \multirow{2}{*}{ Ches of Ommerth } & \multicolumn{5}{|c|}{ 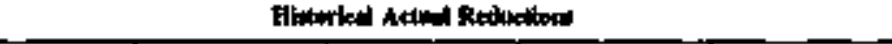 } & \multicolumn{2}{|c|}{ Prokethed heturl Bolloction } \\
\hline & $\operatorname{sen}$ & I9sts & 194. & $19 \%$ & Les & Lith & 201 \\
\hline \multirow[t]{3}{*}{ 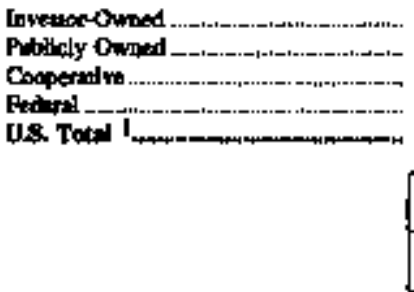 } & $\begin{array}{r}12,330 \\
1,794 \\
2,374 \\
707 \\
17,204\end{array}$ & $\begin{array}{r}16,362 \\
1,898 \\
2,327 \\
2,481 \\
2,4,09\end{array}$ & $\begin{array}{r}1,, 932 \\
2,123 \\
2,459 \\
2,187 \\
1,001\end{array}$ & $\begin{array}{r}2,035 \\
2,568 \\
2,034 \\
2,323 \\
2,591\end{array}$ & $\begin{array}{r}2,064 \\
2,736 \\
2,738 \\
2,358 \\
2,0,95\end{array}$ & $\begin{array}{r}24,661 \\
2,564 \\
2,777 \\
2,358 \\
32,341\end{array}$ & $\begin{array}{r}28,025 \\
3,135 \\
3,209 \\
2,504 \\
3,99\end{array}$ \\
\hline & \multicolumn{5}{|c|}{ 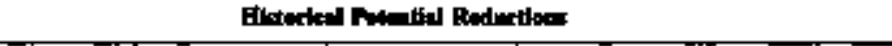 } & \multicolumn{2}{|c|}{ 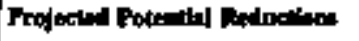 } \\
\hline & 1392 & $1 \% ;$ & 164 & 190 & 1905 & $\mathbf{I b}$ & 201 \\
\hline 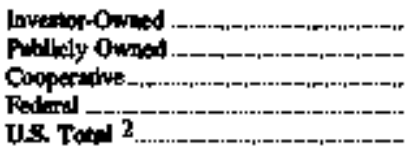 & $\begin{array}{r}23,774 \\
2,306 \\
3,669 \\
2,604 \\
32,40\end{array}$ & $\begin{array}{r}2,059 \\
2,376 \\
4,602 \\
1,411 \\
39,50\end{array}$ & $\begin{array}{r}30,823 \\
2,713 \\
4,783 \\
4,599 \\
4,517\end{array}$ & $\begin{array}{r}34,163 \\
3,252 \\
5,049 \\
4,565 \\
47,509\end{array}$ & $\begin{array}{r}35,068 \\
3,606 \\
5,231 \\
4,438 \\
4,344\end{array}$ & $\begin{array}{r}3,261 \\
3,906 \\
5,3,49 \\
4,458 \\
4,, 35\end{array}$ & $\begin{array}{r}39,691 \\
4,600 \\
6,043 \\
4,604 \\
5,009\end{array}$ \\
\hline
\end{tabular}

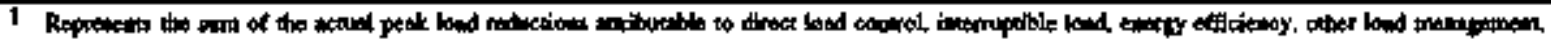
and obe derned-idk mengemen.

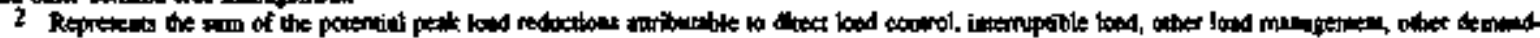

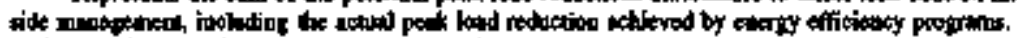

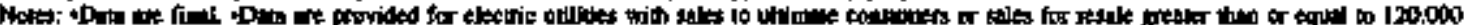

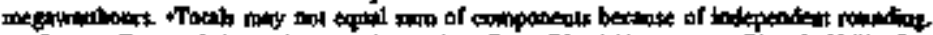

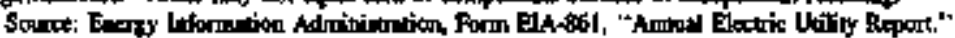


Figure 5. Actual Peak Load Raduction an a Percentage of Total Penck Loid by U.S. Electric Utilities with DSU Peak Load Roduction Programs and by Class of Ownership, 1996

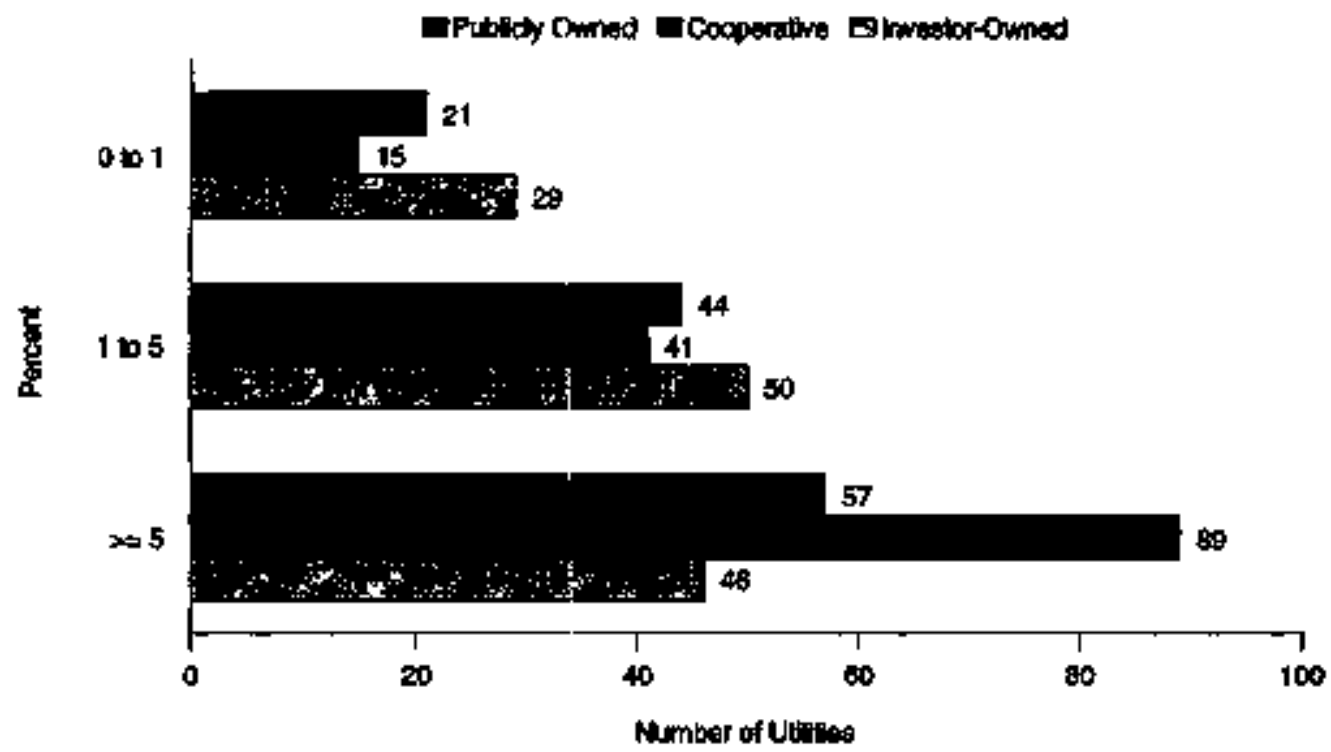

Note: Greph incthdes onty large utilinies thet reponted peak baed reductions.

Sounce: Energy Informatlon Adminkstrallon, Form ElA-661, "Annusal Eloctric Li!lity Report."

Figure 6. The Top 25, 50, and 100 U.S. Electric Utilities with the Greatedt DSM Program Paek Load Reductions by Class of Ownershlp, 1996

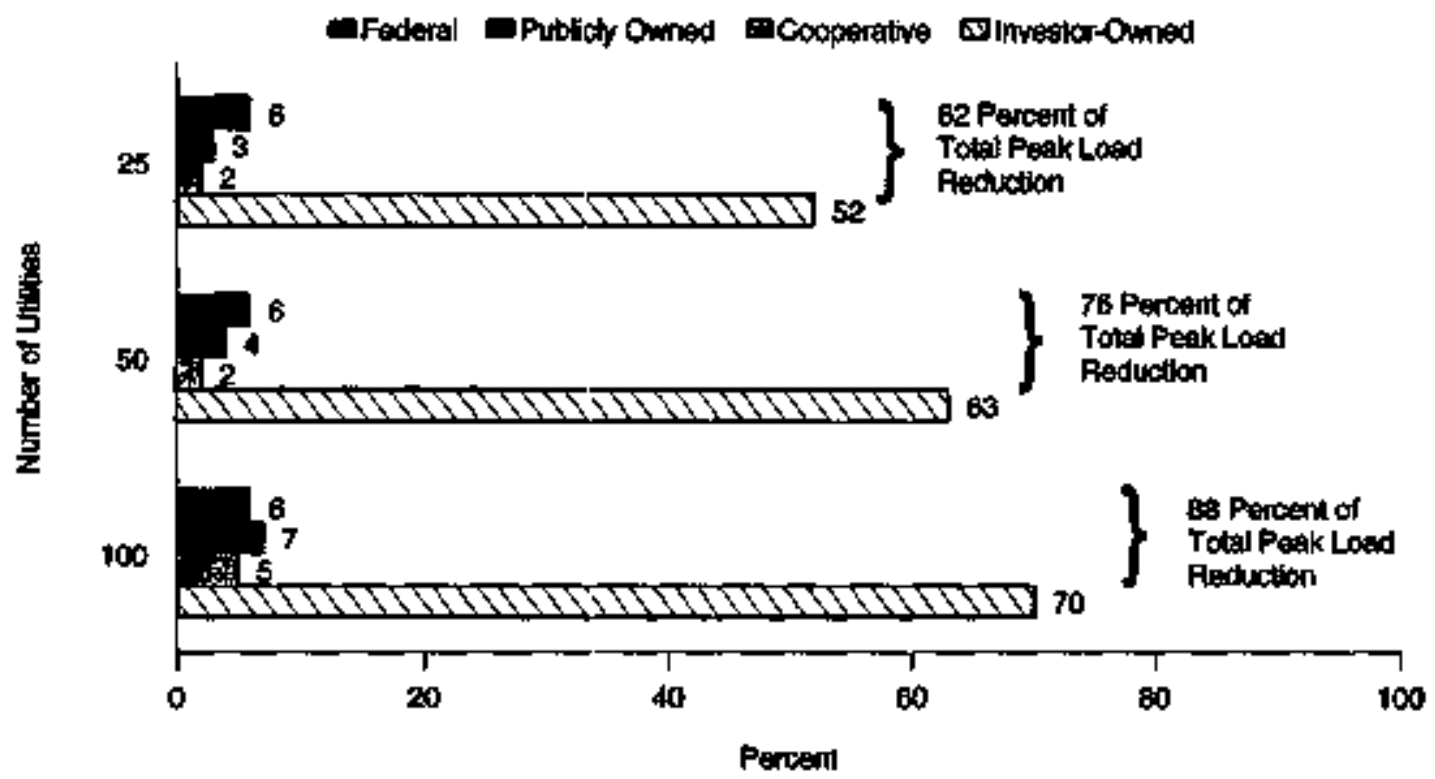

Note: Totats may not equal stem of components because of Independard rounding.

Source: Energy indormation Adminlstration, Form EIA-861, "Anntial Electric Unitly Report" 
Table 13. U.S. Electric Utility Actod and Potential Peak Lowd Redoctions by DSM Progran Category, 1995, 1996, 1997, and 2001

(Megawatts)

\begin{tabular}{|c|c|c|}
\hline \multirow{2}{*}{ 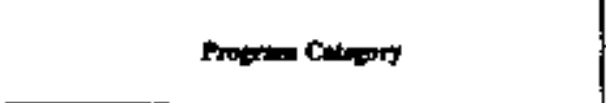 } & \multicolumn{2}{|c|}{ 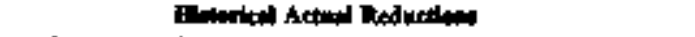 } \\
\hline & 198 & $19 \%$ \\
\hline \multirow[t]{3}{*}{ 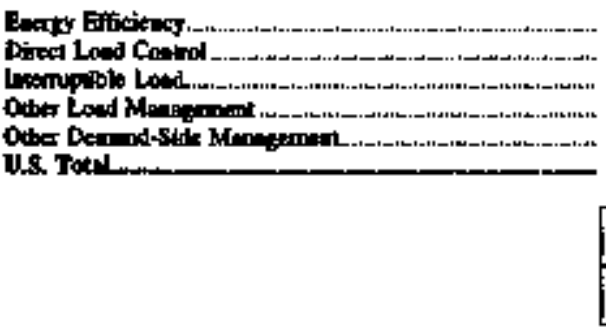 } & $\begin{array}{r}13,212 \\
5,358 \\
2,601 \\
2,168 \\
206 \\
2,51\end{array}$ & $\begin{array}{r}14,243 \\
5,575 \\
7,3,90 \\
2,278 \\
4077 \\
2,803\end{array}$ \\
\hline & \multicolumn{2}{|c|}{ 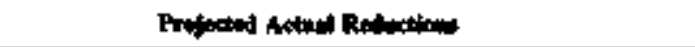 } \\
\hline & $1 \%$ & 2011 \\
\hline \multirow[t]{3}{*}{ 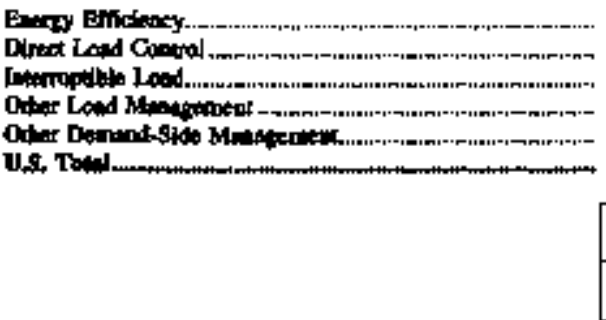 } & 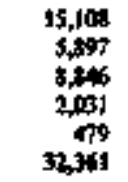 & $\begin{array}{r}17,774 \\
7,140 \\
9,161 \\
2,29 \\
522 \\
36,92\end{array}$ \\
\hline & \multicolumn{2}{|c|}{ 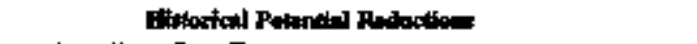 } \\
\hline & 169: & 1096 \\
\hline \multirow[t]{3}{*}{ 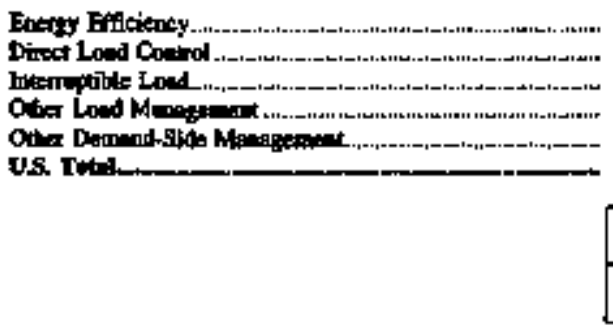 } & $\begin{array}{r}13,212 \\
9,0066 \\
21,520 \\
2,405 \\
476 \\
47,40\end{array}$ & $\begin{array}{r}14,249 \\
9,443 \\
21,558 \\
2,596 \\
500 \\
45,344\end{array}$ \\
\hline & \multicolumn{2}{|c|}{ Prejected Putantin Rtotoutions } \\
\hline & 19 & xy1 \\
\hline 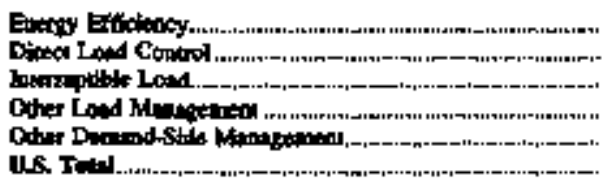 & $\begin{array}{r}15,106 \\
9,813 \\
21,794 \\
2,679 \\
599 \\
49,994\end{array}$ & $\begin{array}{r}17,71 \\
11,444 \\
22,106 \\
2,900 \\
608 \\
34,95\end{array}$ \\
\hline
\end{tabular}

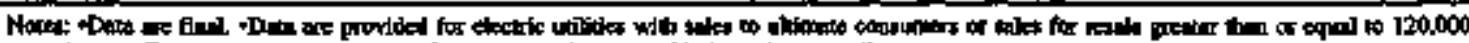

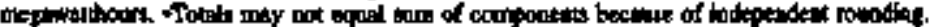

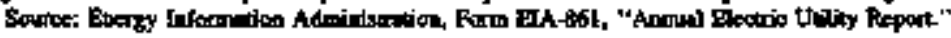


Figure 7. U.8. Electre Utillty Actual and Polential Peak Load Reductione by DSH Program Category, 1998

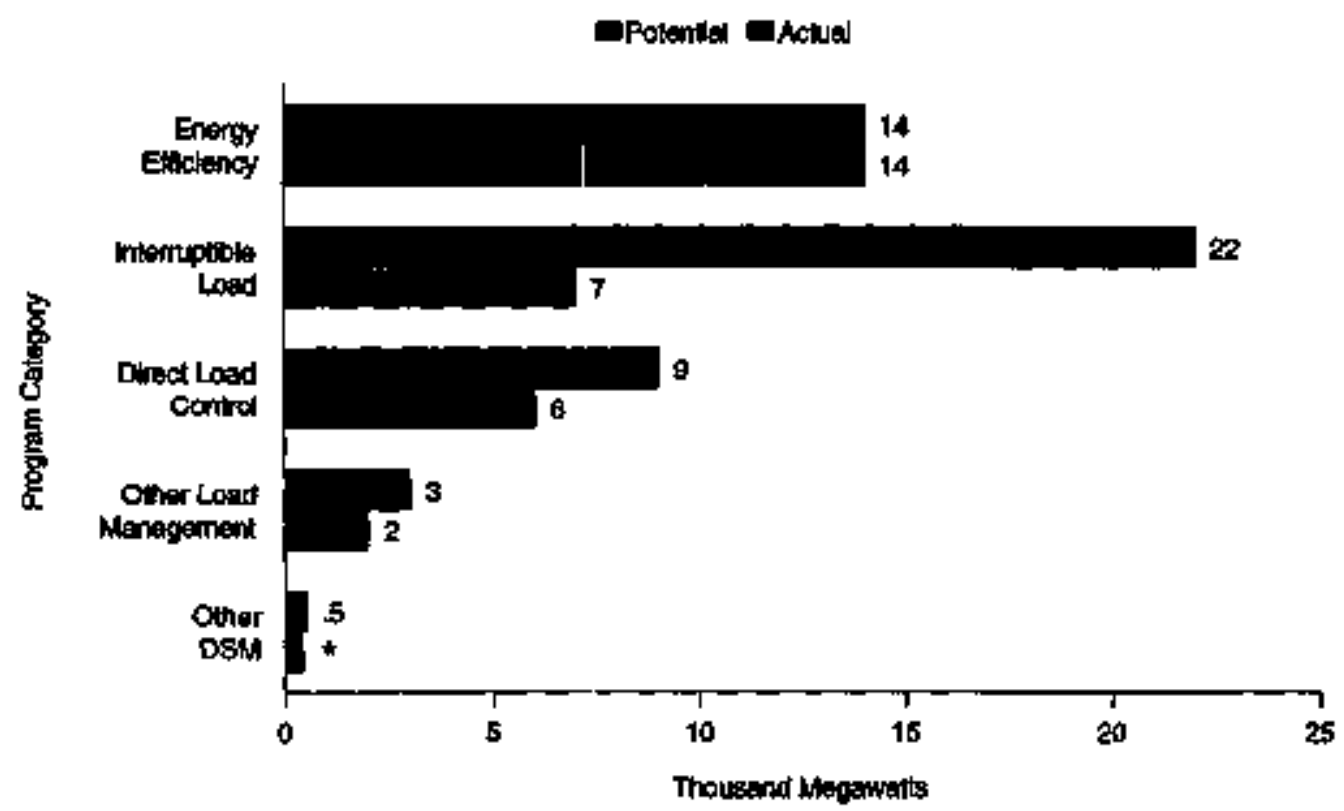

"Value is less than 500 megawatts.

Source: Enargy Informalion Administration, Form ElA-861, "Analual Electritc Utility Feport." 
Table 14. U.S. Electric Utlity Acturl and Potential Peale Lasd Reductions by Sector, 1995 and 1996

(Megawatts)

\begin{tabular}{|c|c|c|c|c|}
\hline \multirow{2}{*}{ Bectors } & \multicolumn{2}{|c|}{ tys } & \multicolumn{2}{|c|}{$19 \%$} \\
\hline & Ath & 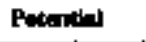 & Motind & Paterited \\
\hline 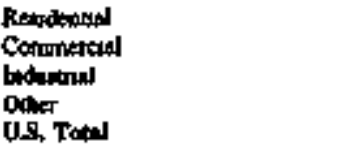 & $\begin{array}{r}10,030 \\
3.054 \\
10,039 \\
548 \\
24841\end{array}$ & $\begin{array}{r}14047 \\
11,494 \\
20,716 \\
373 \\
4,189\end{array}$ & $\begin{array}{r}11,471 \\
8,678 \\
9,043 \\
661 \\
24,853\end{array}$ & $\begin{array}{r}14,691 \\
12,452 \\
20,275 \\
921 \\
4,344\end{array}$ \\
\hline
\end{tabular}

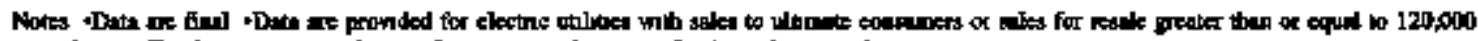

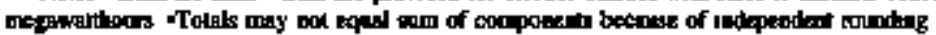

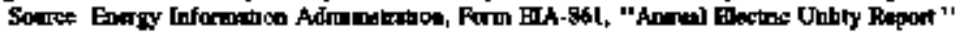

Tabbe 15. U.S. Electric Utilty Incremental Actoal Peak Load Reductions by Class of Ownership, $19 \% 5$ and 1996

(Megawatts)

\begin{tabular}{|c|c|c|c|c|c|c|}
\hline \multirow{2}{*}{ Che of Omertip } & \multicolumn{2}{|c|}{ 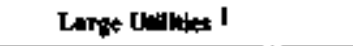 } & \multicolumn{2}{|c|}{2} & \multicolumn{2}{|c|}{ Totel } \\
\hline & $16 \%$ & 1206 & $\cos$ & $19 \%$ & 193 & 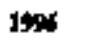 \\
\hline 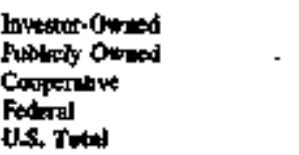 & $\begin{array}{r}3,935 \\
428 \\
224 \\
13 \\
4,400\end{array}$ & $\begin{array}{r}3,115 \\
374 \\
185 \\
16 \\
3,409\end{array}$ & $\begin{array}{r}75 \\
25 \\
10 \\
0 \\
36\end{array}$ & $\begin{array}{c}1 \\
15 \\
17 \\
0\end{array}$ & $\begin{array}{r}3,936 \\
453 \\
234 \\
13 \\
4,45\end{array}$ & $\begin{array}{r}3,115 \\
409 \\
202 \\
16 \\
3,702\end{array}$ \\
\hline
\end{tabular}

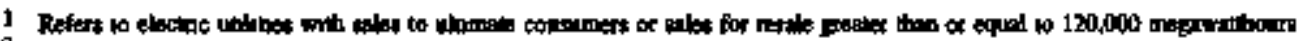

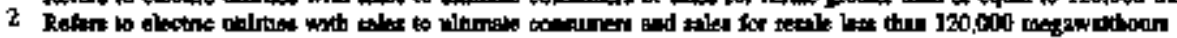

+ Value lass hen 05

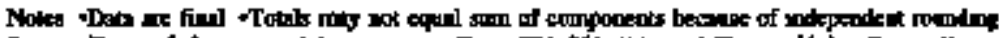

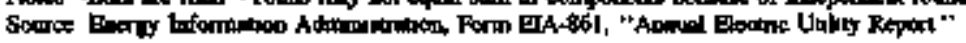


Table 16. U.S. Electric Utilicy Iacrementel Actual Peak Lotod Reductions by DSM Progsram Category, 1995 and 1996

(Megawatus)

\begin{tabular}{|c|c|c|c|c|c|c|}
\hline \multirow{2}{*}{ Propan Camengy } & \multicolumn{2}{|c|}{ 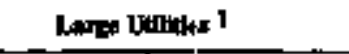 } & \multicolumn{2}{|c|}{ 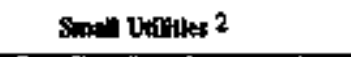 } & \multicolumn{2}{|c|}{ Ther } \\
\hline & 105 & 19:4 & 19N5 & tow & 1ens & IFin \\
\hline 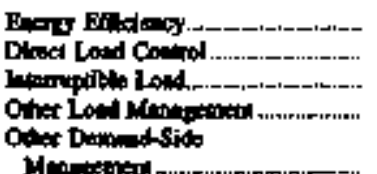 & $\begin{array}{r}1561 \\
552 \\
20209 \\
246\end{array}$ & $\begin{array}{r}1,34] \\
399 \\
1,6 \% 2 \\
19]\end{array}$ & $\begin{array}{r}7 \\
20 \\
4 \\
3\end{array}$ & $\begin{array}{r}2 \\
24 \\
11 \\
9\end{array}$ & $\begin{array}{r}1,567 \\
572 \\
2,213 \\
249\end{array}$ & $\begin{array}{r}1,363 \\
4,72 \\
1,702 \\
200\end{array}$ \\
\hline 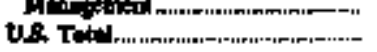 & 40 & 36 & 36 & 52 & 446 & 3,542 \\
\hline
\end{tabular}

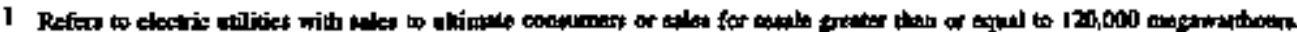

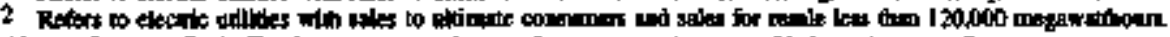

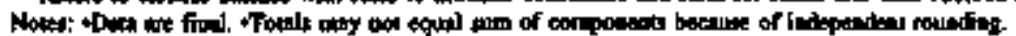

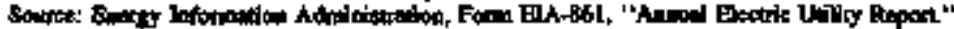

Tahle 17. U.S. Electric Utility Incrementell Actual Peek Loed Reductions by Sector, 1995 and 1996

(Megawatts)

\begin{tabular}{|c|c|c|c|c|c|c|}
\hline \multirow{2}{*}{ Becter } & \multicolumn{2}{|c|}{ tarte tallitied 1} & \multicolumn{2}{|c|}{ Small Cuts 2} & \multicolumn{2}{|c|}{$\mathbf{T}$ 넝 } \\
\hline & wes & 1996 & Ises & 1906 & $19 \%$ & 19\% \\
\hline 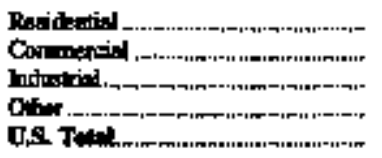 & $\begin{array}{r}860 \\
1,476 \\
2,426 \\
139 \\
466\end{array}$ & $\begin{array}{r}792 \\
953 \\
1,970 \\
93 \\
3,69\end{array}$ & $\begin{array}{r}20 \\
10 \\
4 \\
7 \\
3\end{array}$ & $\begin{array}{r}30 \\
9 \\
8 \\
5 \\
52\end{array}$ & $\begin{array}{r}880 \\
1,186 \\
2430 \\
140 \\
4036\end{array}$ & $\begin{array}{r}922 \\
944 \\
1,978 \\
97 \\
3,76\end{array}$ \\
\hline
\end{tabular}

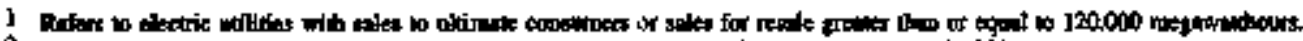

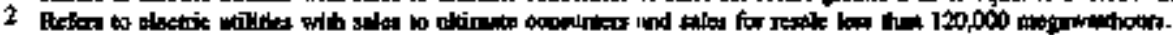

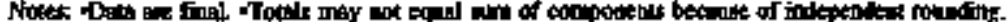

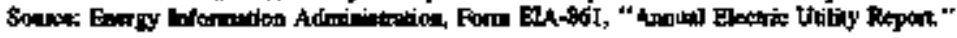


Table 18. US. Electric Utility Actual and Potential Peak Loend Reductions by North Antericam Electric Retiability Coancil Rezion and Bnwatt by Utility, 1995, 1996, 1997, and 2001

(Megawatts)

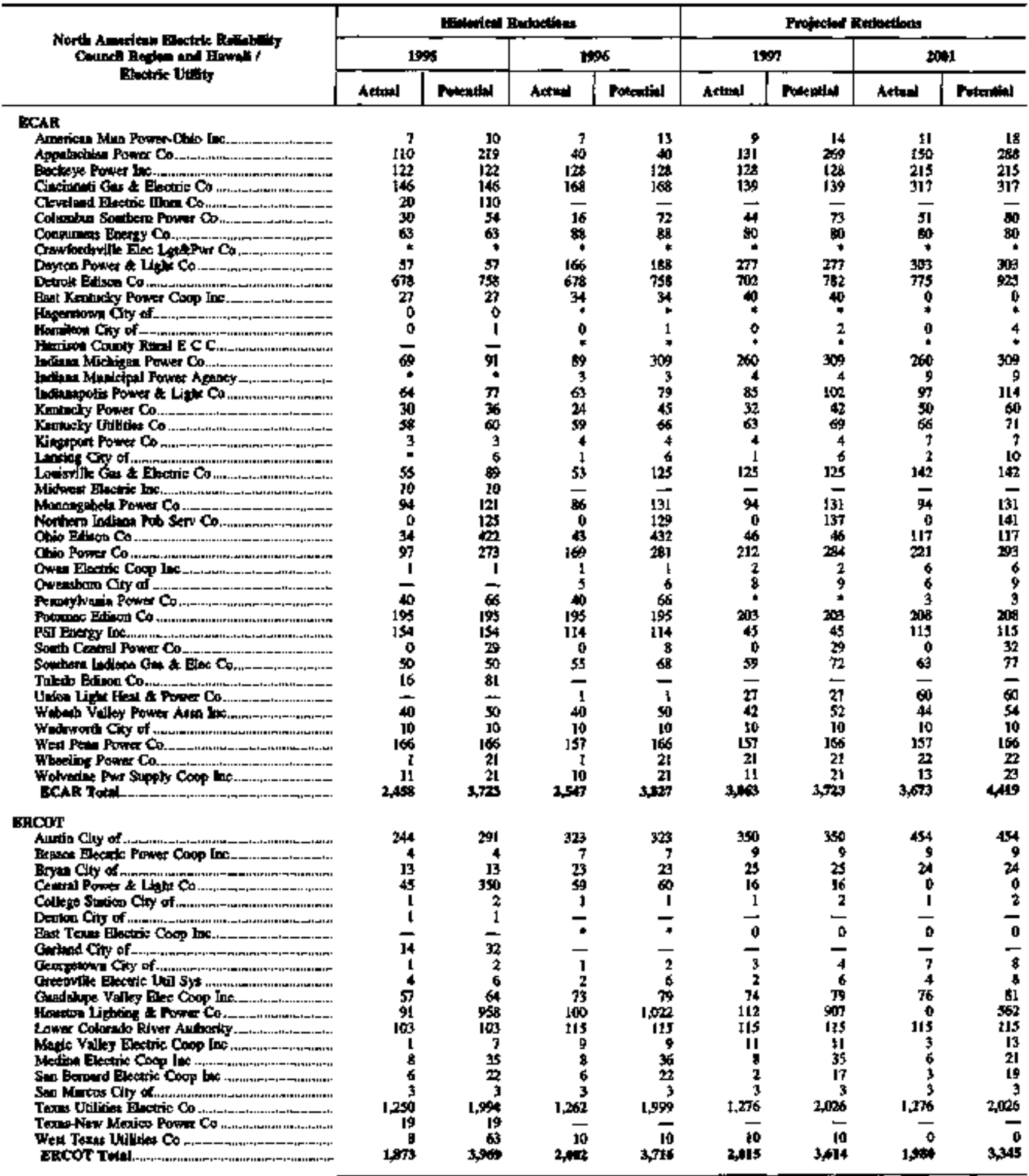

See footnotes an and of bolol. 
Table 18. U.S. Electric Utlity Acturl and Potenthal Peals Load Reductions by North Amerteon Blectric Reliablity Council Region and Eawail by Uthity, 1995, 19\%, 1997, and 2001 (Megawatts) (Contunued)

\begin{tabular}{|c|c|c|c|c|c|c|c|c|}
\hline \multirow{3}{*}{ 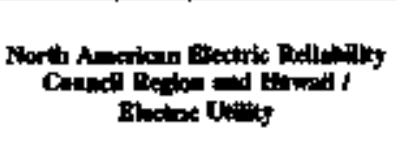 } & \multicolumn{4}{|c|}{ 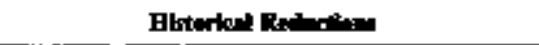 } & \multicolumn{4}{|c|}{ 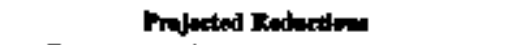 } \\
\hline & \multicolumn{2}{|c|}{$19 \%$} & \multicolumn{2}{|c|}{$19 \%$} & \multicolumn{2}{|c|}{$I \% 9$} & \multicolumn{2}{|c|}{2001} \\
\hline & Actrill & Prinomint & Aalnal & Pultatul & I I I & 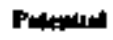 & 1) & Patedialla \\
\hline
\end{tabular}

MAdC

A N N Eame Coop

Adm: Eloctio Cacp las

Alkthay Ehatine Coop lac

Alante Ory Electe Co

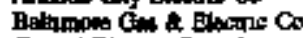

Cetrial Elate Coop bo

Copenty Bloctenc Coop los

Chintack Ruril Filec Coop be

Delwire Blectinc Coop Int

telmern Points \& 1dpe co

Eurom Uthat: Coenm

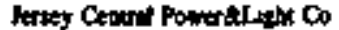

Mtropotion Edroo Co

Nodirmestan Rril E C A loc

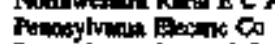

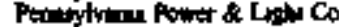

Potonec Finctic Pow a Ca

Puble Servat Eextodiche Co

PECO Enogy Co

Somernot Rurris Elac Cocp Inc

Sownen Horylud El Coop Inc

Solpmit Cepted R B C Corp

Th-Cooncy Rot Elac Coop the

Inind Electer Coop inc

Valley Rond Eloxinc Cocp lac

MAdC Totel

1
25
42
95
65
4
7
5
8
145
795
250
7
64
23
364
280
49
3
37
0
2
4
4
710

2
27
45
96
676
5
15
6
27
276
4
603
280
1
64
313
636
407
389
3
216
3
3
4
5
415

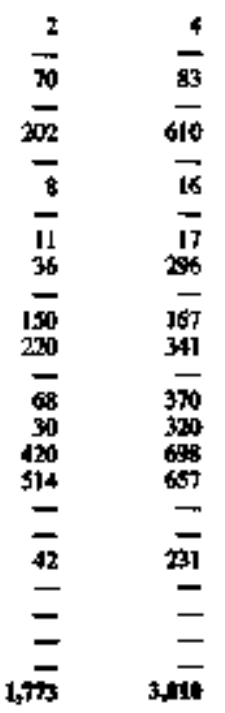

$\frac{1}{6}$

2

1

MAN

Boune gloctur Copp

Cain Iforas Leth Ca

Coles-doning Electine CoOp

Cohnibea $C$ ing if

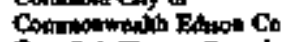

Con Belk Eloxirc Code Ine

Orrme Brver bectne Coep inc

Fanten Illin Flentine Coop

Fermegon Cyy of

Innow Power Co

Whdion on \& Eloctite 6

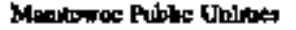

Hant fild ory of

neliod Electuc Coop.

Stution Eectoc Coop Inc

Soumenten D. Eles Coop lac

Sontminater Blocent Coop Ins

Spoteptetd Coy of

Tn-Coumb finetac Cow he

Smo Electinc Co

Waym. What Countes Bue Cocp

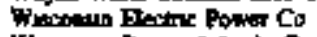

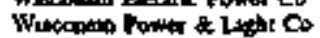

Wieconin fubte Powt Ix \$Ys

Wuccosin Fuble Sarwe Cop

MAN Todel

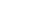

4h1

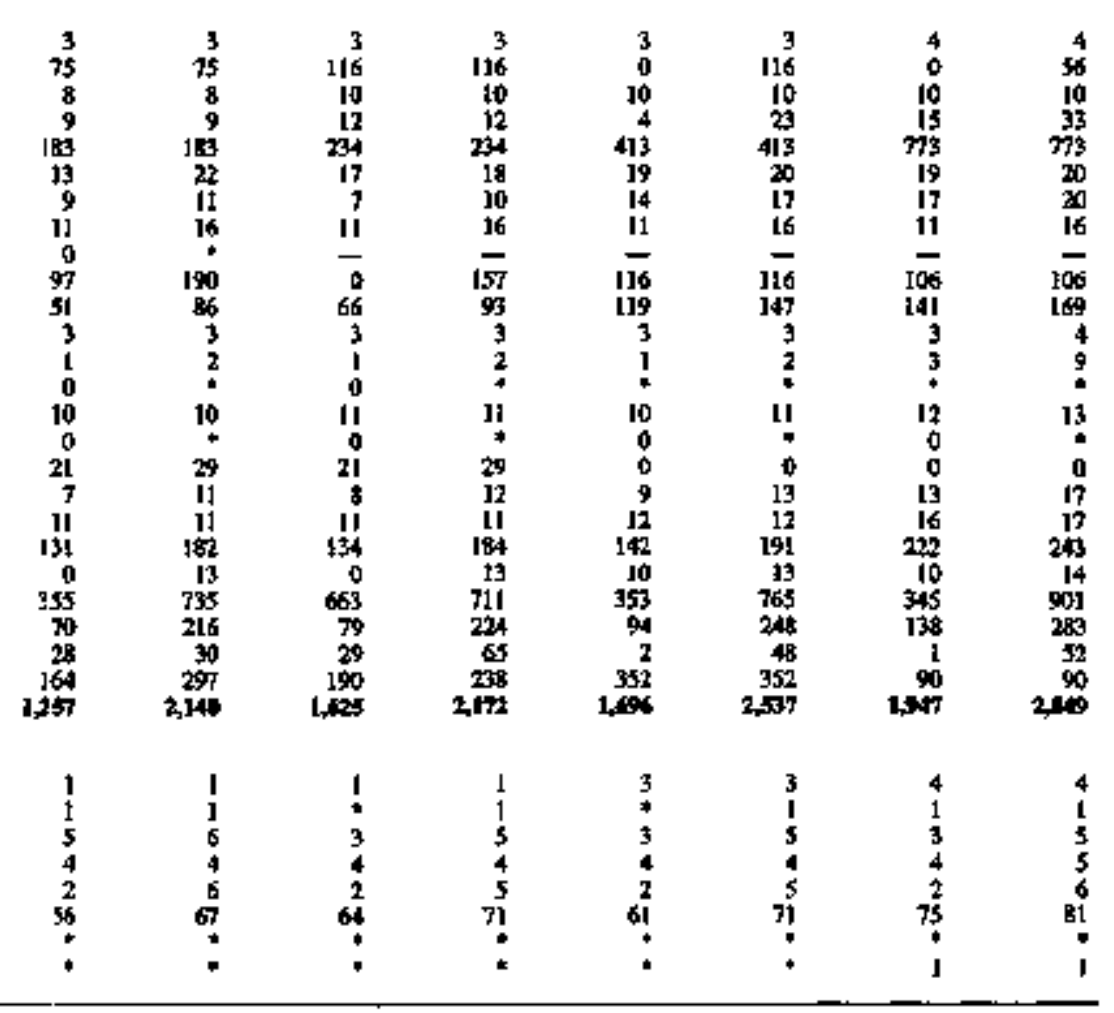

Sex foctoctes it and of thib 
Table 18. US. Electric Utllity Actual and Potenthal Peol: Load Redections by North American Electric Rellabllity Coumcil Region and Fawall by Utility, $1995,1996,1997$, and 2001 (Megawatts) (Continued)

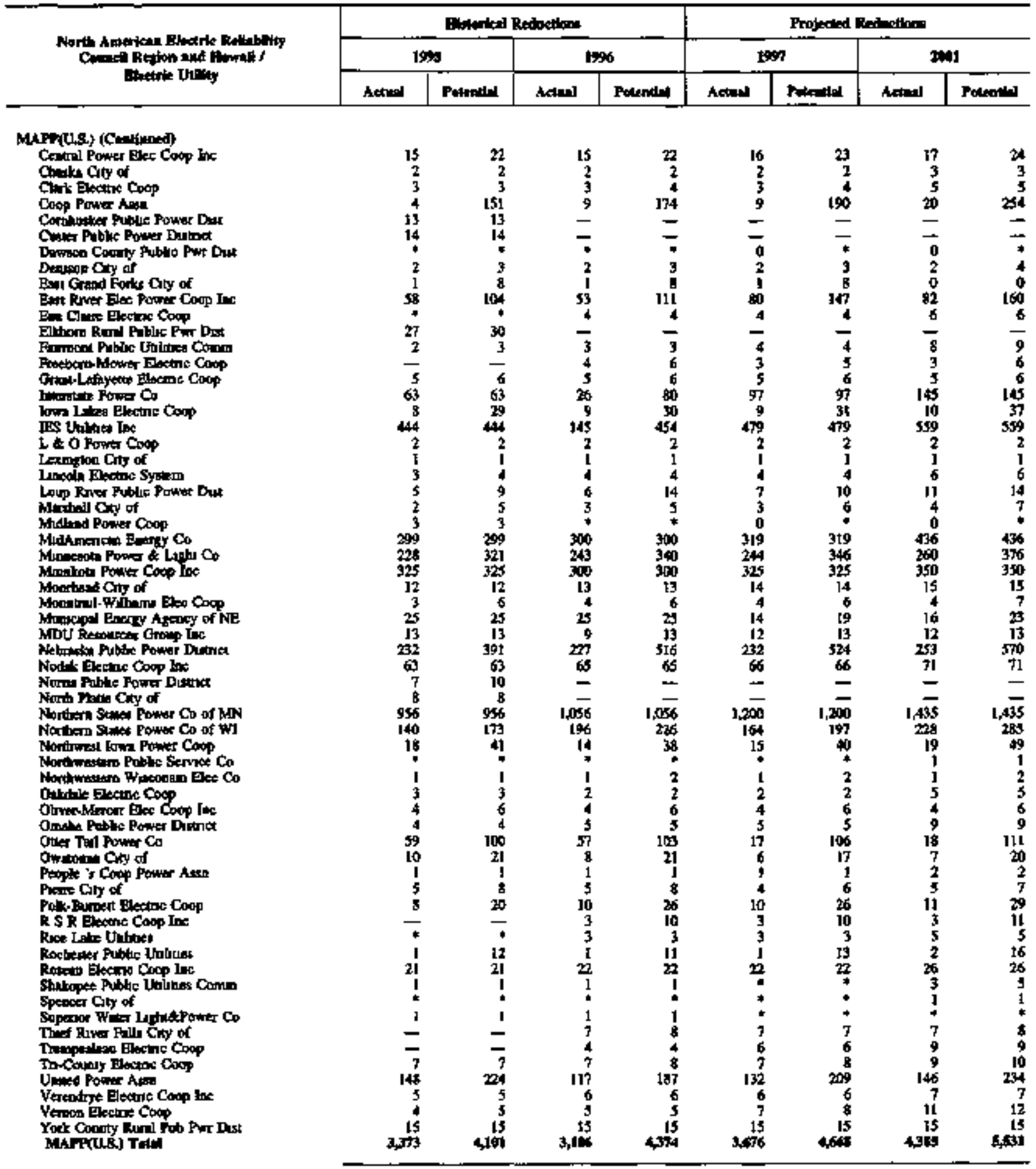


Table 18. U.S. Flectric Utility Actuol and Potestial Peak Loed Reduetions by Nonth Americon Electric Reliability Conneil Region and Howatl by Utility, 1995, 1996, 1997, and 2001

(Megawatts) (Continued)

\begin{tabular}{|c|c|c|c|c|c|c|c|c|}
\hline \multirow{3}{*}{ 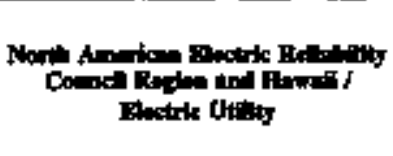 } & \multicolumn{4}{|c|}{ 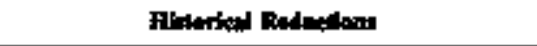 } & \multicolumn{4}{|c|}{ Projeted Rumatime } \\
\hline & \multicolumn{2}{|c|}{10} & \multicolumn{2}{|c|}{$19 *$} & \multicolumn{2}{|c|}{$19 \%$} & \multicolumn{2}{|c|}{201} \\
\hline & Anul & 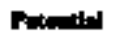 & Act & Potint" & Antel & Poweotid & net-l & 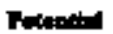 \\
\hline
\end{tabular}

Noctus

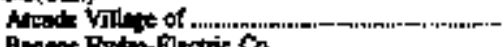

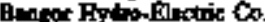

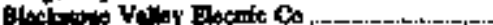

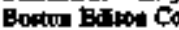

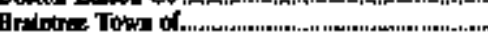

Butintion Cly of.

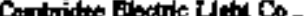

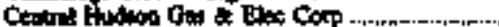

Contrid Mrine Powp Co

Central Varoned Pob Sery Corp...m......................

Gilopest oty of

Clkims botitito Co

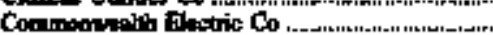

Concest Elettic Co

Connecticol Lithr \& Pourer Co

Comactionl Varley Blac

Conoolidiod Bding CoNy be

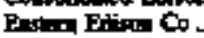

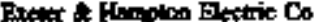

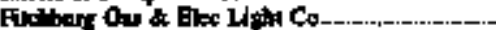

Granto $5 t-5$ Eectic Co....

Grees Momptodo Power Corp

Hivitam Dits of

Hotyole thy of.

tontem Cily of

Linteton Tow of

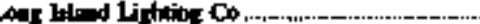

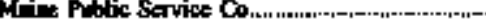

Mrapachactin Ebctile Co

Mancily Trown of

Montide Electio Co.

Nen Fontind Power Co.

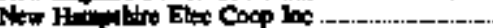

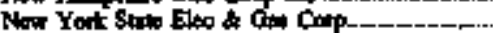

Nouport Electrde Corp.

Nitosim Momank Pooper Corp.

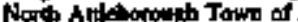

Numood Ciky of

Omyra hes.

Drangs 8 Roctland Lidls he

Fones Arthority of Stre of NY

tubtic Skrtion co of N

Reting Towe of

Rochentar Cos a Elextric Conp

Shrembint Tom of

Tuatue chy of

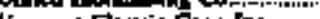

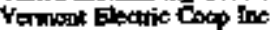

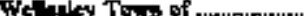

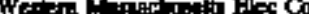
ac

Alkan Dratrie Cong be.

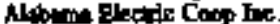

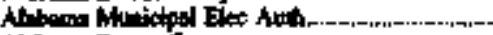

Alibarm Prese Co.

Albende City of ........m-.........

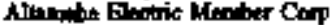

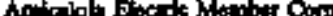

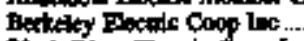

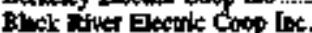

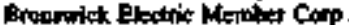

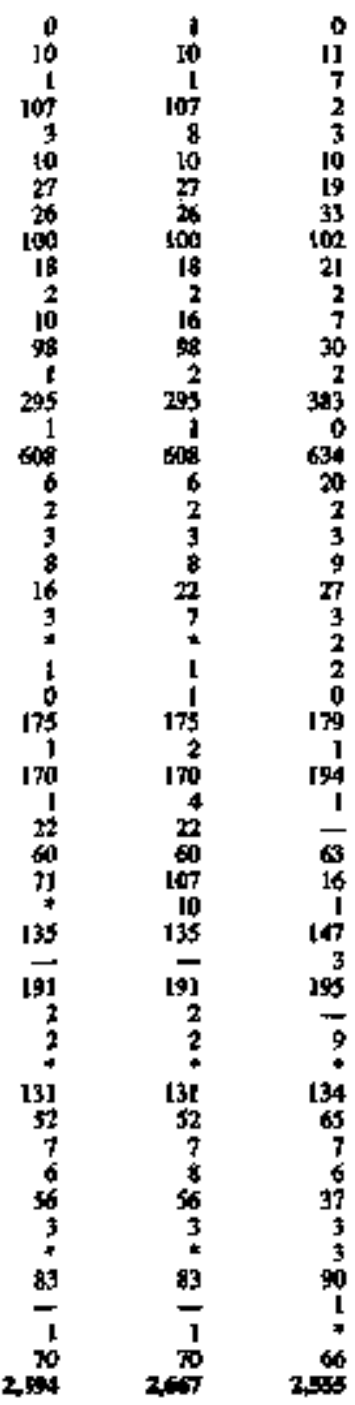

$\begin{array}{rrr}5 & 5 & 5 \\ 10 & 305 & 38 \\ 3 & 5 & 3 \\ 9 & 823 & 98 \\ 3 & 4 & 4 \\ 2 & 6 & 2 \\ 30 & 40 & 33 \\ 5 & 5 & 23\end{array}$

I

14

6

11

20

131

2

7

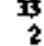

282

0
457

457

2

1

2

4

a

16

23

$\frac{1}{75}$

75

143

4

$\div$

14

79

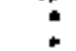

7

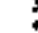

17

3

$2 \pi$

2,52

$\begin{array}{rrr}1 & 0 & \\ 21 & 10 & 1 \\ 10 & 17 & 17 \\ 8 & 6 & \\ 8 & 5 & 11 \\ 11 & 11 & 23 \\ 23 & 25 & 25 \\ 31 & 251 & 131 \\ 131 & 0 & \end{array}$

\section{I}

10

$\begin{array}{rr}5 & 5 \\ 135 & 13 \\ 599 & 7 \\ 8 & 101 \\ 8 & + \\ 4 & 2 \\ 6 & 30 \\ 6 & 6\end{array}$

5
113
4
5
9
4
4
4
6
29

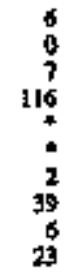

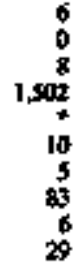

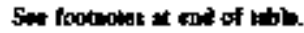


Tahle 18. US. Electrie Vitility Actual and Potential Peak Loed Reductions by North Ameriten Electric Reliahllity Coancll Reghon and Fawai by Utility,

1995, 1996, 1997, and 2001

(Megawatts) (Continued)

\begin{tabular}{|c|c|c|c|c|c|c|c|c|}
\hline \multirow{3}{*}{ 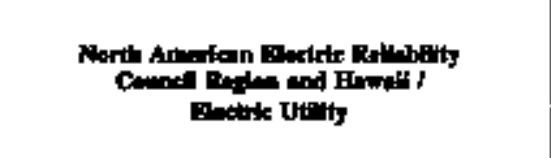 } & \multicolumn{4}{|c|}{ 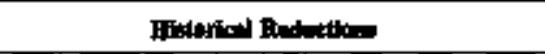 } & \multicolumn{4}{|c|}{ Projecton Redenthon } \\
\hline & \multicolumn{2}{|c|}{1095} & \multicolumn{2}{|c|}{ Lss } & \multicolumn{2}{|c|}{$\mathbf{6 9 7}$} & \multicolumn{2}{|c|}{$\operatorname{sen}$} \\
\hline & Anthes & Patriatled & Antul & Pelentid & Acted & Plenifill & Axtad & Peterfall \\
\hline \multicolumn{9}{|l|}{$\operatorname{sinc}\left(\mathrm{C}_{n+1}\right)$ ath } \\
\hline HARC Bintrif Coop [ec., & 2 & $\mathbf{2}$ & 2 & 2 & $\boldsymbol{2}$ & 2 & 2 & 2 \\
\hline Cender City of & & - & & & 3 & 4 & 4 & 4 \\
\hline 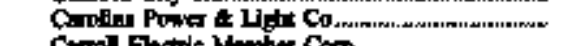 & 1,143 & 1,149 & 1,174 & 1,17 & 665 & 1,160 & 206 & 1.211 \\
\hline Comol Eloctide Menber Cog & 17 & 24 & $\dot{2}$ & & 0 & 0 & 0 & 0 \\
\hline 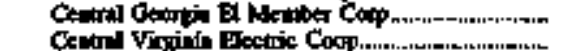 & 19 & $\frac{2}{n}$ & $\begin{array}{l}\mathbf{2 1} \\
\mathbf{8}\end{array}$ & $\stackrel{22}{96}$ & $\begin{array}{l}17 \\
\text { [01 }\end{array}$ & $\begin{aligned} 18 \\
112\end{aligned}$ & 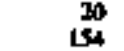 & 21 \\
\hline Chootan & I & I & I & 1 & 2 & 2 & 2 & 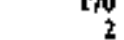 \\
\hline Clay Electe Goop lnk & 62 & 127 & 59 & 130 & 66 & 117 & 78 & 137 \\
\hline 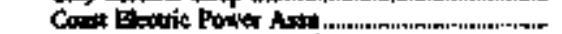 & 20 & $\mathbf{n}$ & 20 & 20 & 20 & 20 & 30 & 30 \\
\hline 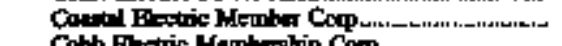 & 4 & 4 & $\overline{10}$ & $\overline{19}$ & - & - & $\bar{c}$ & - \\
\hline Cabb Elatric Nombarripip Corp & 55 & 59 & 19 & 19 & 10 & í & [ & I \\
\hline 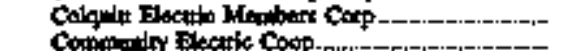 & 21 & 21 & $\begin{array}{l}9 \\
4\end{array}$ & $\begin{aligned} 34 \\
4\end{aligned}$ & 10 & 26 & 11 & 32 \\
\hline Convelof & 35 & 10 & 20 & $\$ 0$ & 21 & 41 & $\overrightarrow{\mathbf{w}}$ & 10 \\
\hline 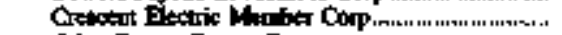 & 13 & 17 & - & - & - & - & $=$ & - \\
\hline 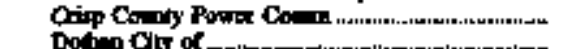 & 2 & 2 & $\stackrel{0}{5}$ & 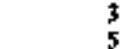 & 0 & 3 & & 4 \\
\hline 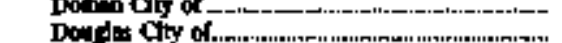 & 4 & 3 & 3 & 3 & 3 & 9 & 4 & 14 \\
\hline 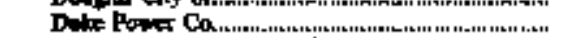 & 83 & 1,000 & \% & 1,206 & 101 & 1,216 & 136 & $1,24]$ \\
\hline 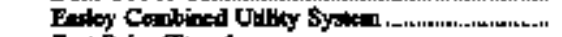 & I1 & 11 & î & 11 & 12 & 12 & 12 & 12 \\
\hline Est Potax Olyy of & 4 & 9 & 7 & i2 & 6 & 6 & וה & 12 \\
\hline 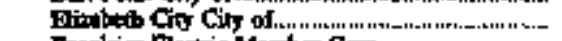 & 0 & 2 & 0 & 4 & 0 & 4 & 0 & 5 \\
\hline 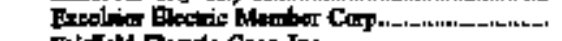 & $\mathbf{0}$ & 3 & a & 4 & 0 & 4 & 0 & 4 \\
\hline 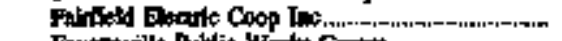 & 3 & 3 & 4 & 4 & $\$$ & 4 & 4 & 4 \\
\hline 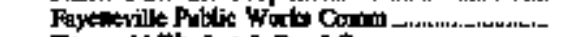 & I & I & $\mathbf{0}$ & a & 0 & 0 & 0 & 0 \\
\hline Efrigrald Whe Lef \& Bood Canm & I & 1 & 1 & $\mathbf{J}$ & I & I & i & j \\
\hline 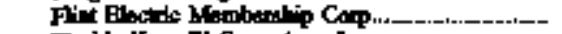 & 40 & 40 & $\mathbf{s}$ & $\mathbf{5}$ & 3 & $\mathbf{s}$ & 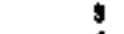 & 8 \\
\hline 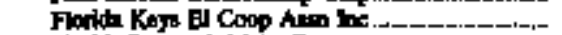 & & & 3 & & 3 & 4 & 4 & 6 \\
\hline 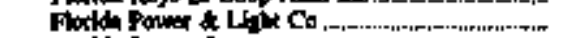 & $1, \pi i$ & נונד & 2,005 & 2,005 & 2,53 & 2,153 & 2,733 & 2733 \\
\hline Foxide power cop - & $1,3 \% 6$ & 1,614 & 1,539 & 1.935 & 1,989 & 1,989 & 2,213 & 2,213 \\
\hline 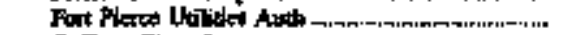 & & & & & - & 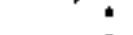 & * & $*$ \\
\hline 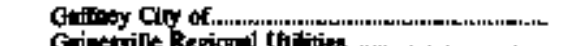 & 1 & 1 & $\underset{16}{1}$ & $\frac{1}{16}$ & I & 1 & 1 & t's \\
\hline 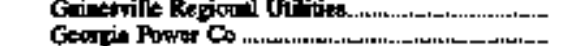 & 845 & sis & 16 & $\begin{aligned} 16 \\
579\end{aligned}$ & 906 & $\begin{array}{l}16 \\
906\end{array}$ & 936 & 18 \\
\hline Graty Condy Five Momber Corp. & 5 & 7 & $\mathbf{I}$ & 2 & 3 & 7 & 5 & 7 \\
\hline Oretrilik Utibles Compo & 27 & 3) & 4 & 5) & 4 & 54 & 49 & s\% \\
\hline 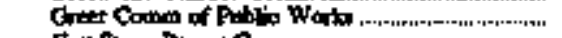 & 1 & 1 & 4 & 4 & 4 & 4 & 4 & 4 \\
\hline 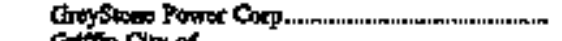 & 25 & 49 & 2s & $\$ 0$ & 26 & si & 28 & 36 \\
\hline Grtinh Cyly of & $\overline{163}$ & $\overrightarrow{163}$ & $\begin{array}{r}7 \\
174\end{array}$ & $\stackrel{2}{1}$ & 2 & $\frac{2}{2}$ & 2 & 2 \\
\hline 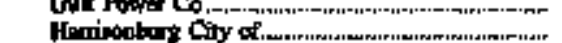 & 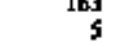 & $\begin{array}{r}103 \\
5\end{array}$ & 3 & 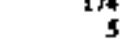 & 14 & 14 & 26 & 34 \\
\hline 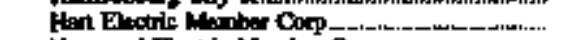 & 7 & 8 & $\overrightarrow{7}$ & 8 & 1 & 8 & F & is \\
\hline Heyrood Elextric Momber Carp & 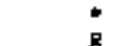 & إ & 4 & 8 & 0 & 0 & 0 & 0 \\
\hline 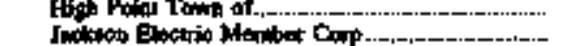 & $\begin{array}{r}8 \\
49\end{array}$ & 75 & 10 & $\begin{array}{l}76 \\
49\end{array}$ & 10 & 76 & 53 & 36 \\
\hline 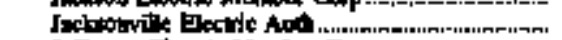 & is & 15 & 17 & 17 & $\mathbf{3}$ & 101 & 137 & 2002 \\
\hline 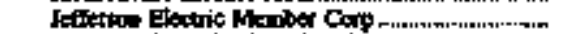 & 12 & 14 & 12 & 14 & 13 & 14 & $\mathbf{L}$ & 16 \\
\hline c Merter Copp ......................... & - & - & 12 & 33 & 14 & 36 & 19 & 30 \\
\hline of $\ldots$ & 17 & i7 & 20 & 20 & t8 & is & tg & t\$ \\
\hline Kinitamer Utily Anthouty ............................. & 3 & js & 3 & 20 & 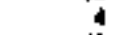 & 24 & 6 & 41 \\
\hline lowedod $C_{\text {y }}$ of & 40 & 44 & 45 & 49 & 48 & 53 & 64 & 70 \\
\hline ectric Mamberilyp Corp................ & $\mathbf{I}$ & i & I & $\mathbf{]}$ & I & I & I & I \\
\hline 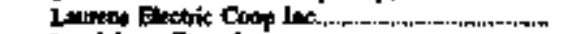 & & 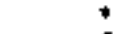 & & 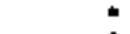 & $*$ & * & * & $*$ \\
\hline 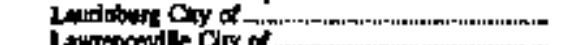 & 3 & 4 & 3 & 3 & 3 & & 3 & 4 \\
\hline 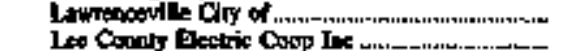 & 64 & 6 & 68 & $\stackrel{4}{7}$ & 76 & 8 & 83 & 4 \\
\hline leverg Gy af & 4 & 4 & It & 12 & 11 & 12 & 12 & 13 \\
\hline beriop Cllyy of & 2 & $\xi$ & 2 & 5 & 2 & 5 & 0 & 0 \\
\hline 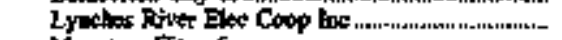 & 4 & 4 & 4 & d & 3 & 3 & 3 & 3 \\
\hline Niven of of of & 2 & 2 & 2] & 21 & 20 & 20 & 22 & $\mathbf{2 2}$ \\
\hline Mtarioth Ciy of & $\bar{j}$ & 7 & - & - & - & - & $=$ & $=$ \\
\hline 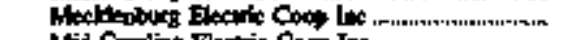 & 8 & 13 & 14 & 16 & 15 & 19 & $\mathbf{1 8}$ & 24 \\
\hline 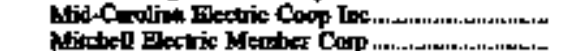 & $\begin{array}{l}9 \\
0\end{array}$ & $\begin{array}{l}9 \\
9\end{array}$ & 10 & $\begin{array}{l}10 \\
10\end{array}$ & $\begin{array}{r}11 \\
0\end{array}$ & II & $\begin{array}{r}12 \\
0\end{array}$ & 12 \\
\hline & & & & & & & & \\
\hline
\end{tabular}

Sect foomons an and of roble. 


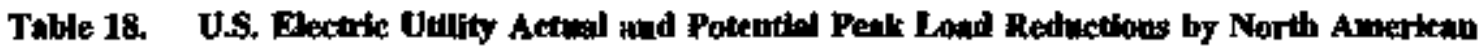
Electric Relinbilty Council Region and Hawait by Utilty,

$1995,1996,1997$, and 2001

(Megawarts) (Continued)

\begin{tabular}{|c|c|c|c|c|c|c|c|c|}
\hline \multirow{3}{*}{ 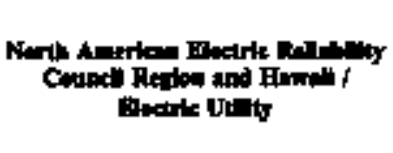 } & \multicolumn{4}{|c|}{ 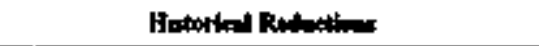 } & \multicolumn{4}{|c|}{ 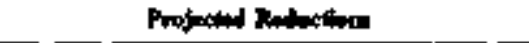 } \\
\hline & \multicolumn{2}{|c|}{ 19s:s } & \multicolumn{2}{|c|}{596} & \multicolumn{2}{|c|}{19} & \multicolumn{2}{|c|}{201} \\
\hline & Axind & Tecoting & Acted & 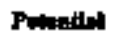 & Aetar & Molnoth & 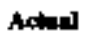 & $P=1$ delle \\
\hline
\end{tabular}

BRPC (C)

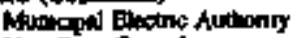

New Bin oy of

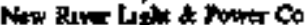

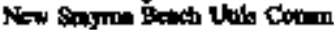

Nembety Cry ol

Newin Wis Sever \& lsght Conn

North Carchan Eowters M P A

Muth Croln li Memher Corp

North Crolnas Whon Fower Ager,

Nonthers Nest Hec Coop be

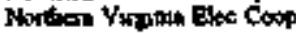

Geph Cry of

Onnative Electer Meonber CoT

Orngutsy ofy of

Oringio Uthiluc Coots

Pimeno Elactac Codp lne

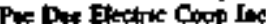

Plater Elextric Neother Coxp

Pripa Oeorge Derine Coop

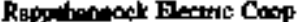

Rayle Eloctin Mambenthip CoP

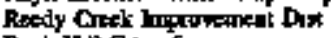

Rock fill City of

Rocky Moont Cry of

S-nit: Rimat Etec Member Cor

Sovm in Flene Pawt Co

Somme Elocine benters Cosp

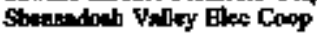

Stoment Raver Elec Power Asan

Stontits Tom of

Sotrping Shodt Ex Member Coop

Sowt Cordion Bloctat

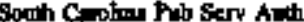

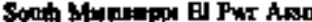

Solihwde Eetinc Cocp inc

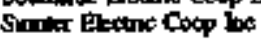

Stromene Vally Eec Coop kx

Talls anese Coy of

Thone Eleme Co

Thenene Yaluy Autocky

Thomenalie Cory of

Tudelond Flectinc Mientur Corp

TruCanary Bloc Momber Cor

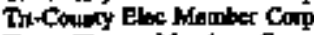

Thon Elecoric Menuters Cop

Unom city of

Vurame Election \& Poptr Co

Worion Flectinc Merober Comp

Wanhergton Cits of

Whitoon Diny of

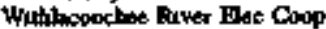

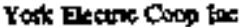

Stenc Told

SIPP

Alfati Eletrac Coop las

Altan Oty of

Artanses Elext Coop Corp

Bnley Coury Eloc Coop AII

C\& L Eectic Coop Corp

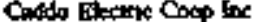

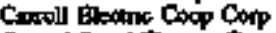

Centril Rral bectoc Cocp

Cooktone Hill Ele Chop Inte

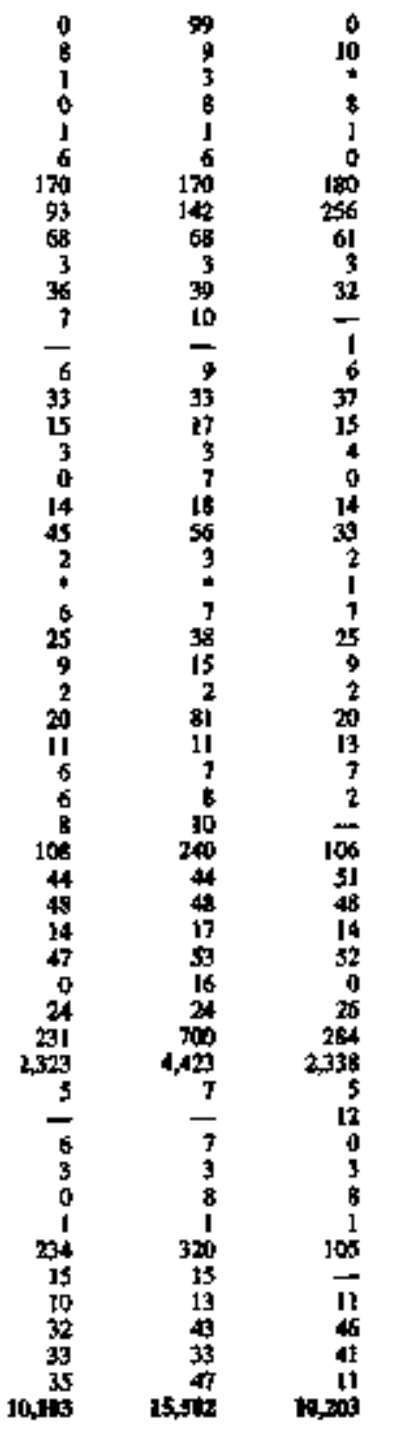

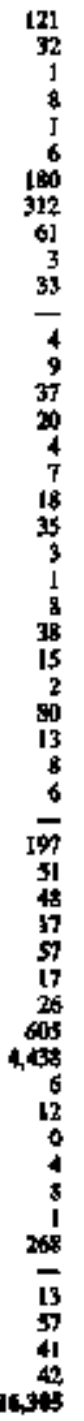

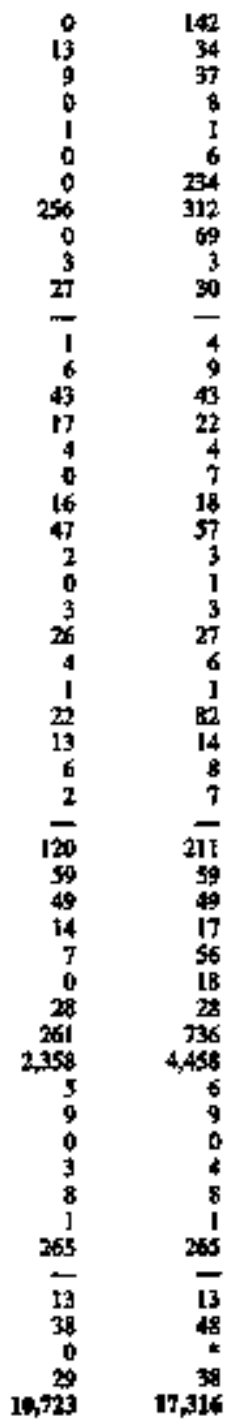

$\begin{array}{rr}0 & 196 \\ 15 & 39 \\ 9 & 37 \\ 0 & 10 \\ 1 & 1 \\ 0 & 6 \\ 0 & 249 \\ 266 & 525\end{array}$

37

10

1304

Sin foolocisy at end of eble

\begin{tabular}{|c|c|c|c|c|c|c|c|}
\hline $\begin{array}{l}3 \\
1\end{array}$ & $\begin{array}{r}4 \\
2 \\
\mathbf{5 2 9}\end{array}$ & $\begin{array}{l}3 \\
0 \\
0\end{array}$ & $\begin{array}{r}4 \\
500\end{array}$ & $\begin{array}{l}3 \\
1 \\
0\end{array}$ & $\begin{array}{r}4 \\
529\end{array}$ & $\begin{array}{l}4 \\
2 \\
0\end{array}$ & $\begin{array}{r}5 \\
529\end{array}$ \\
\hline & 35 & - & - & - & - & - & - \\
\hline 1 & 2 & I & 2 & I & 3 & 1 & 3 \\
\hline 8 & 26 & 11 & 19 & 11 & 13 & 11 & 13 \\
\hline 10 & 75 & 5 & 75 & 10 & 75 & 10 & 80 \\
\hline & 7 & 5 & 1 & 6 & 7 & 7 & 8 \\
\hline & 25 & g & 2B & 9 & 30 & 10 & 37 \\
\hline
\end{tabular}


Table 18. US. Elactric Utilty Actuol and Potential Peak Lond Rethetions by North American Flectric Rellabllity Council Region and Hawai by Utilty, 1995, 1996, 1997, and 20101

(Megawratts) (Continued)

\begin{tabular}{|c|c|c|c|c|c|c|c|c|}
\hline \multirow{3}{*}{ 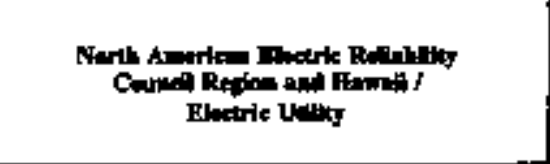 } & \multicolumn{4}{|c|}{ 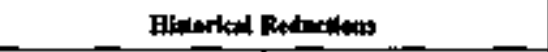 } & \multicolumn{4}{|c|}{ 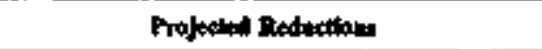 } \\
\hline & \multicolumn{2}{|c|}{ Les } & \multicolumn{2}{|c|}{$\operatorname{sen}$} & \multicolumn{2}{|c|}{$\operatorname{lem}$} & \multicolumn{2}{|c|}{201} \\
\hline & Aclanl & Poteriled & Antual & Potentivil & Actin & Pumenting & Actod & Ponewitim \\
\hline 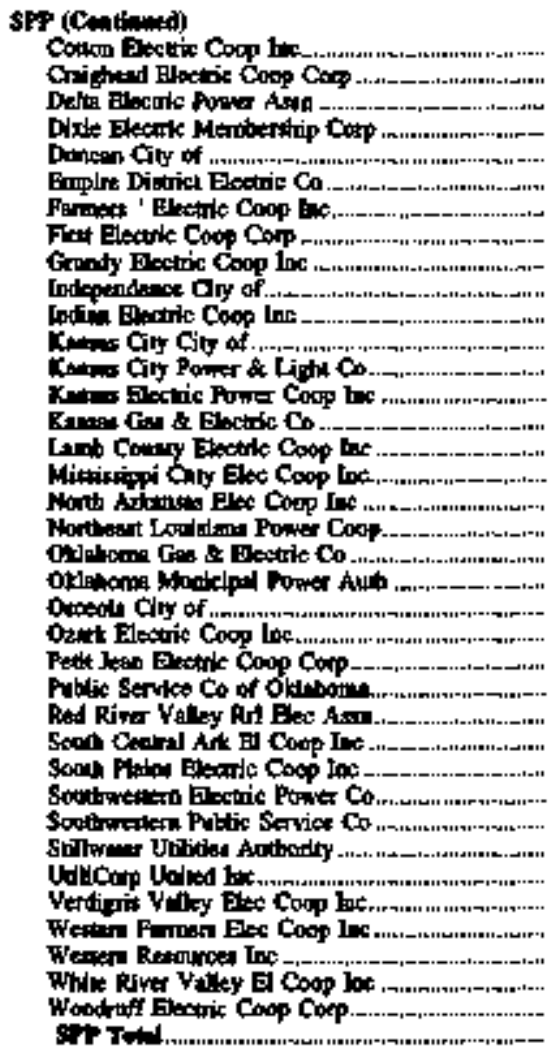 & $\begin{array}{r}78 \\
6 \\
14 \\
38 \\
8 \\
18 \\
73 \\
3 \\
0 \\
34 \\
34 \\
10 \\
2 \\
5 \\
3 \\
29 \\
1 \\
3 \\
2 \\
3 \\
84 \\
6 \\
5 \\
6 \\
10 \\
90 \\
1 \\
10 \\
15 \\
0 \\
15 \\
15 \\
21 \\
744\end{array}$ & $\begin{array}{r}5 \\
26 \\
7 \\
16 \\
* \\
38 \\
8 \\
29 \\
5 \\
6 \\
35 \\
34 \\
34 \\
190 \\
399 \\
5 \\
5 \\
429 \\
1 \\
3 \\
2 \\
3 \\
112 \\
5 \\
5 \\
25 \\
55 \\
132 \\
1 \\
10 \\
15 \\
53 \\
166 \\
22 \\
56 \\
2669\end{array}$ & $\begin{array}{r}0 \\
3 \\
2 \\
14 \\
4 \\
21 \\
5 \\
22 \\
2 \\
4 \\
3 \\
33 \\
34 \\
93 \\
12 \\
0 \\
2 \\
5 \\
3 \\
231 \\
1 \\
4 \\
2 \\
3 \\
97 \\
2 \\
5 \\
5 \\
13 \\
168 \\
1 \\
0 \\
15 \\
0 \\
132 \\
16 \\
30 \\
924\end{array}$ & $\begin{array}{r}0 \\
21 \\
46 \\
7 \\
32 \\
6 \\
41 \\
2 \\
6 \\
6 \\
39 \\
31 \\
43 \\
167 \\
6 \\
41 \\
5 \\
5 \\
431 \\
1 \\
4 \\
2 \\
3 \\
71 \\
8 \\
5 \\
21 \\
13 \\
306 \\
1 \\
0 \\
16 \\
47 \\
170 \\
27 \\
59 \\
269\end{array}$ & $\begin{array}{r}0 \\
8 \\
2 \\
14 \\
4 \\
20 \\
5 \\
22 \\
3 \\
6 \\
3 \\
0 \\
34 \\
42 \\
12 \\
0 \\
3 \\
5 \\
4 \\
163 \\
5 \\
4 \\
0 \\
2 \\
55 \\
3 \\
5 \\
12 \\
45 \\
164 \\
1 \\
0 \\
16 \\
0 \\
19 \\
0 \\
28 \\
738\end{array}$ & $\begin{array}{r}6 \\
22 \\
4 \\
16 \\
4 \\
25 \\
6 \\
41 \\
3 \\
6 \\
7 \\
39 \\
34 \\
54 \\
197 \\
6 \\
404 \\
5 \\
5 \\
380 \\
4 \\
4 \\
2 \\
3 \\
70 \\
8 \\
7 \\
21 \\
110 \\
320 \\
6 \\
5 \\
16 \\
47 \\
170 \\
0 \\
57 \\
2764\end{array}$ & $\begin{array}{r}0 \\
9 \\
2 \\
16 \\
32 \\
5 \\
23 \\
6 \\
6 \\
6 \\
0 \\
0 \\
48 \\
12 \\
0 \\
3 \\
5 \\
4 \\
91 \\
5 \\
5 \\
0 \\
3 \\
3 \\
4 \\
8 \\
19 \\
0 \\
179 \\
1 \\
9 \\
15 \\
0 \\
19 \\
0 \\
29 \\
595\end{array}$ & $\begin{array}{r}6 \\
24 \\
4 \\
18 \\
32 \\
6 \\
43 \\
8 \\
9 \\
10 \\
33 \\
0 \\
60 \\
193 \\
6 \\
408 \\
5 \\
6 \\
316 \\
5 \\
5 \\
2 \\
3 \\
3 \\
10 \\
9 \\
34 \\
0 \\
335 \\
1 \\
10 \\
16 \\
47 \\
170 \\
0 \\
69 \\
254\end{array}$ \\
\hline 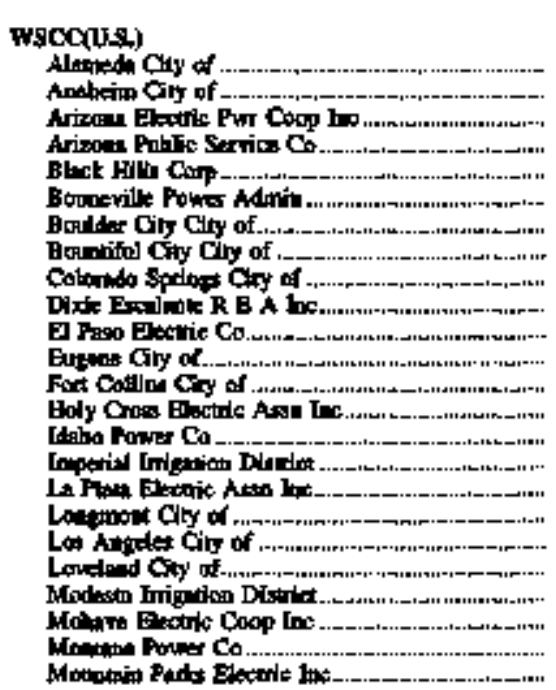 & 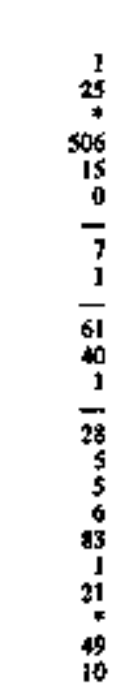 & 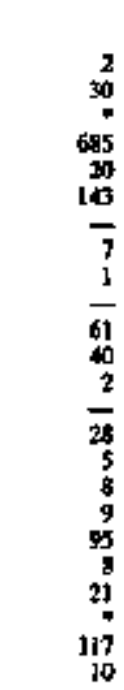 & $\begin{array}{r}2 \\
21 \\
1 \\
506 \\
0 \\
5 \\
7 \\
4 \\
66 \\
44 \\
2 \\
10 \\
0 \\
6 \\
5 \\
5 \\
86 \\
2 \\
39 \\
57 \\
51\end{array}$ & $\begin{array}{r}2 \\
25 \\
1 \\
685 \\
-0 \\
5 \\
57 \\
5 \\
66 \\
44 \\
10 \\
0 \\
6 \\
9 \\
8 \\
98 \\
8 \\
64 \\
57 \\
57\end{array}$ & $\begin{array}{r}2 \\
20 \\
6 \\
78 \\
78 \\
0 \\
5 \\
7 \\
4 \\
4 \\
2 \\
45 \\
2 \\
0 \\
0 \\
7 \\
5 \\
6 \\
82 \\
7 \\
39 \\
1 \\
60 \\
62\end{array}$ & $\begin{array}{r}2 \\
25 \\
8 \\
797 \\
70 \\
5 \\
7 \\
9 \\
77 \\
45 \\
2 \\
0 \\
4 \\
9 \\
9 \\
94 \\
8 \\
65 \\
1 \\
50 \\
12\end{array}$ & $\begin{array}{r}3 \\
44 \\
99 \\
90 \\
0 \\
6 \\
5 \\
5 \\
0 \\
60 \\
2 \\
0 \\
0 \\
5 \\
0 \\
7 \\
79 \\
7 \\
0 \\
1 \\
91 \\
14\end{array}$ & $\begin{array}{r}3 \\
50 \\
11 \\
727 \\
0 \\
5 \\
5 \\
10 \\
0 \\
60 \\
2 \\
0 \\
0 \\
4 \\
11 \\
91 \\
8 \\
0 \\
11 \\
91 \\
14\end{array}$ \\
\hline
\end{tabular}

seo footinotat at end of whe. 
Tahit 18. U.S. Electric Utility Acturl and Potential Pegk Logd Reductions by North American Electric Relialbitity Conncil Region and Hawall by Uenty, $1995,1996,1997$, and 2001

(Megawatts) (Contunued)

\begin{tabular}{|c|c|c|c|c|c|c|c|c|}
\hline \multirow{3}{*}{ 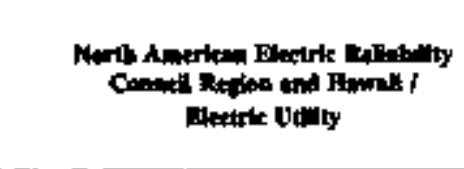 } & \multicolumn{4}{|c|}{ 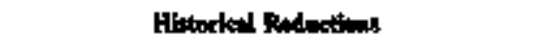 } & \multicolumn{4}{|c|}{ 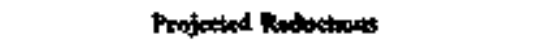 } \\
\hline & \multicolumn{2}{|c|}{$\operatorname{ses}$} & \multicolumn{2}{|c|}{ 1936 } & \multicolumn{2}{|c|}{ 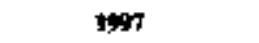 } & \multicolumn{2}{|c|}{201} \\
\hline & Actral & ramentel & Anted & Pathether & Aetral & Pasprallot & Adthe & Pmentiol \\
\hline \multicolumn{9}{|l|}{ 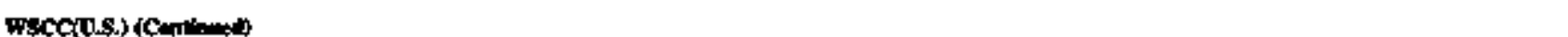 } \\
\hline 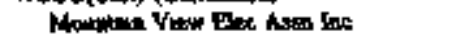 & - & - & 29 & 46 & 37 & 62 & $\$ 3$ & 94 \\
\hline Nowapento Bactore Coop IAc & $\mathbf{8}$ & 33 & 9 & 15 & 9 & 16 & JI & 20 \\
\hline Neroln Power Co & 36 & 43 & 33 & 33 & 33 & 33 & is & 15 \\
\hline Ontrim Fower Dustera No 5 & * & 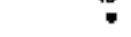 & - & - & - & - & - & . \\
\hline Puefic Ges \& Fotinc Co & 1.126 & 1,183 & 1,119 & 1.176 & 1,176 & 1.248 & {$[, 136$} & 1,248 \\
\hline Pufforp & 0 & 375 & 0 & $\$ 71$ & a & 0 & 0 & 0 \\
\hline Pato Alio Coty of & 6 & 6 & 6 & 6 & 6 & 6 & 8 & 8 \\
\hline Puscent Cxy of & 4 & 6 & 7 & 7 & 7 & 3 & $\$ 6$ & 16 \\
\hline Poude Viloy R E A loc & - & - & $\mathbf{I}$ & 1 & 2 & 2 & $\mathbf{z}$ & 3 \\
\hline Pulde Samax Co of Colpondo & 216 & 273 & 209 & 466 & 67 & 20 & 65 & 305 \\
\hline 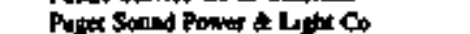 & $\mathbf{0}$ & 38 & 0 & $\pi$ & $\mathbf{0}$ & 72 & 0 & 72 \\
\hline PUD No 1 of Bencos County & 1 & I & I & 1 & 2 & 2 & 2 & 2 \\
\hline PUD No I of Cant Conty & 0 & a & 5 & 9 & 0 & 0 & 0 & 0 \\
\hline PUD No I af Pand Omilo Coty & 1 & $\mathbf{j}$ & I & $j$ & 1 & I & I & j \\
\hline PUD Na 2 of G ant County & \$i & $\$$ & 62 & 62 & 92 & 92 & 94 & 94 \\
\hline Rodthet Coy af & 29 & II & 30 & 30 & 31 & 39 & 42 & 42 \\
\hline Ruentide Coty $\alpha$ & 12 & 12 & - & - & - & - & - & - \\
\hline Roterile Dity of & 4 & 4 & $\mathbf{s}$ & $\mathbf{s}$ & $\mathbf{s}$ & $\$$ & 7 & 7 \\
\hline 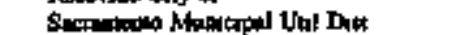 & 402 & $\mathbf{4 0}$ & 429 & $\mathbf{4 2 0}$ & 446 & 446 & $51 \%$ & 518 \\
\hline Snll Ruvar Proy At J \& P Du & 234 & 225 & 136 & 22 & 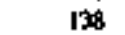 & 236 & 198 & 260 \\
\hline 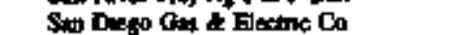 & IBI & jot & 243 & 243 & 252 & 280 & 322 & 320 \\
\hline 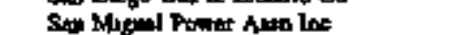 & - & - & 1 & 1 & 1 & I & 3 & 3 \\
\hline $\operatorname{sen} C \cos Q y+$ of & 6 & 8 & 7 & ]1 & 7 & II & 10 & 15 \\
\hline Semtio Cty of & 27 & 27 & 60 & 60 & 66 & 60 & 90 & 90 \\
\hline Sum Pacile Poner Co & 47 & T & 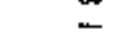 & - & $\rightarrow$ & - & - & - \\
\hline 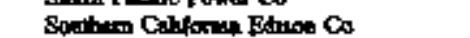 & 1,509 & 3,536 & 1,614 & 3,500 & 1,62 & 3,965 & 1.622 & 3,968 \\
\hline Sprngietd Coy of & 3 & 3 & 4 & 4 & 1 & I & 3 & 3 \\
\hline Solpher Spmog; Valky E C he & 2 & 2 & 2 & 2 & 2 & $\mathbf{2}$ & 2 & 2 \\
\hline Then Eledtic Corp be & $\mathbf{i}$ & 2 & i & 4 & 1 & 4 & 0 & 0 \\
\hline Tweon Eloctinc Ponva Co & 33 & 33 & 38 & 38 & 139 & 139 & 165 & 165 \\
\hline 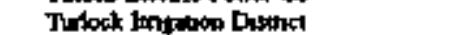 & 9 & 9 & 2 & 4 & I & 3 & i & 3 \\
\hline Unted Power los & 12 & is & $\mathbf{s}$ & II & 13 & 13 & 22 & 29 \\
\hline Utath Muncepal Power Axesy & $\mathbf{J}$ & I & I & 1 & I & ] & 2 & 1 \\
\hline Vert Imporou Destact of is & $\tau$ & B & - & $m$ & - & - & - & - \\
\hline Venrob ciry of & 8 & 5 & 8 & L & * & 16 & $\mathbf{z}$ & 17 \\
\hline 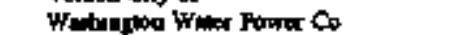 & $\$$ & $m$ & 90 & 90 & 104 & 104 & 139 & 139 \\
\hline Yellowitane Volley Elec Co-bp & 7 & 7 & & I & g & 9 & 13 & 13 \\
\hline wbctuts.) Tht & 5.110; & 3,90 & $5, \mathbf{1 0}$ & $8,7+8$ & 5,34 & $\mathbf{4} \mathbf{4}$ & 5,57 & 1,, 80 \\
\hline Cantpoos U.s. & 2040 & 47000 & $2 \theta$ & 46. 401 & 32,394 & Q145 & $36 ; 89$ & Solpt: \\
\hline \multicolumn{9}{|l|}{ NSCC } \\
\hline 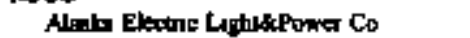 & 7 & 7 & 1 & 14 & 4 & 12 & 5 & $\mathbf{1 3}$ \\
\hline 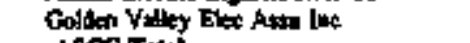 & 2 & 2 & 2 & 2 & 2 & $\mathbf{2}$ & 1 & 1 \\
\hline ASCE Tati & 9 & • & 7 & 15 & 6 & 14 & 6 & 34 \\
\hline \multicolumn{9}{|l|}{ Bhw } \\
\hline Haver Elextre Ligh Co Ins & $\mathbf{1}$ & $\mathbf{I}$ & 3 & B & 5 & 10 & 9 & 14 \\
\hline Harpont Electict Co he & 3 & 3 & 5 & 5 & 8 & B & $\mathbf{0}$ & 0 \\
\hline Man Elene Co Lad & g & ta & 9 & 15 & 12 & $1 ?$ & 14 & 24 \\
\hline Homill Totat & 15 & 19 & 17 & 28 & 24 & 35 & ts & 3 \\
\hline Us. THA & 20,561 & 47,20 & $+4,03$ & $\mathbf{4 0 ; 3 4 4}$ & 32,361 & 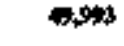 & $3 ; 4,4)$ & sisto: \\
\hline
\end{tabular}

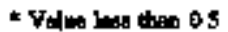

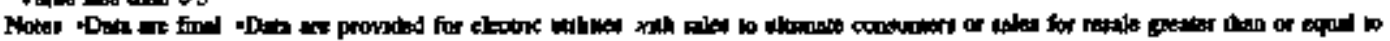

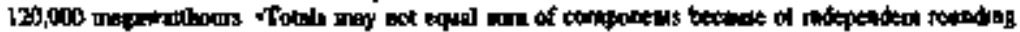

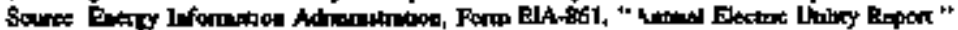


Table 19. U.S. Elextric Utity Actual Peak Lood Reductions by North American Electric Relibility Councll Reglon and Hawnit by DSM Program Category, 1996 (Megawatts)

\begin{tabular}{|c|c|c|c|c|c|c|}
\hline 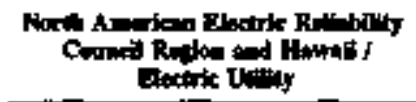 & Bundery & Dhret Lood & biterquble & OAlud land & 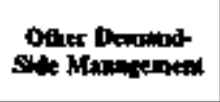 & Tod DsM \\
\hline 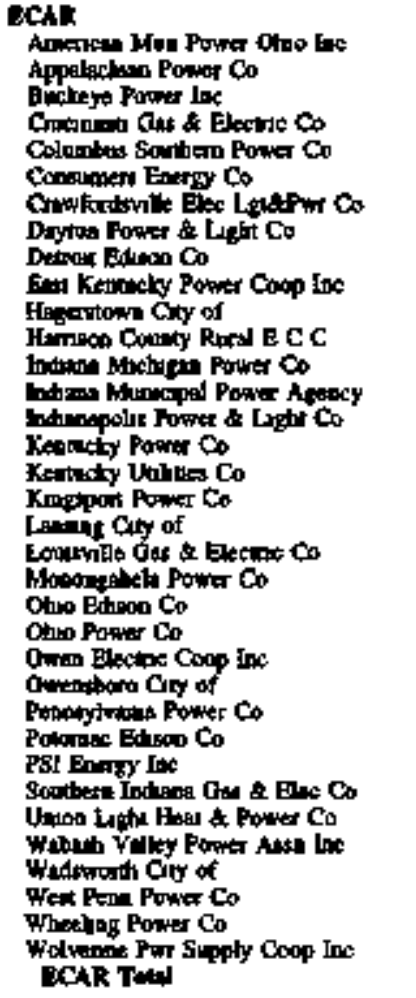 & 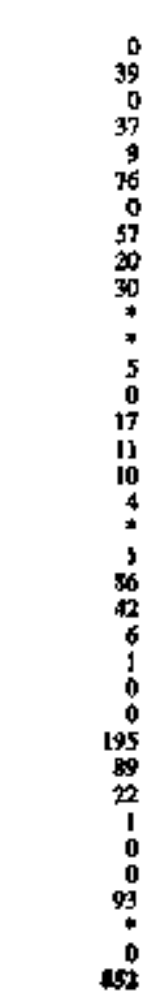 & $\begin{array}{r}0 \\
0 \\
125 \\
25 \\
0 \\
1 \\
0 \\
0 \\
159 \\
0 \\
0 \\
0 \\
0 \\
3 \\
0 \\
0 \\
0 \\
0 \\
0 \\
0 \\
0 \\
0 \\
+ \\
0 \\
0 \\
0 \\
0 \\
0 \\
33 \\
1 \\
40 \\
0 \\
0 \\
0 \\
10 \\
39\end{array}$ & $\begin{array}{r}7 \\
0 \\
0 \\
106 \\
3 \\
0 \\
0 \\
109 \\
500 \\
0 \\
0 \\
0 \\
80 \\
0 \\
0 \\
13 \\
34 \\
0 \\
0 \\
52 \\
0 \\
0 \\
151 \\
0 \\
0 \\
40 \\
0 \\
24 \\
0 \\
0 \\
0 \\
10 \\
0 \\
0 \\
0 \\
0 \\
1,120\end{array}$ & $\begin{array}{r}1 \\
0 \\
0 \\
4 \\
3 \\
0 \\
0 \\
0 \\
4 \\
0 \\
4 \\
4 \\
0 \\
1 \\
0 \\
8 \\
0 \\
0 \\
0 \\
0 \\
1 \\
12 \\
0 \\
0 \\
0 \\
0 \\
0 \\
0 \\
0 \\
0 \\
0 \\
65 \\
1 \\
0 \\
100\end{array}$ & $\begin{array}{l}0 \\
0 \\
0 \\
0 \\
0 \\
7 \\
0 \\
0 \\
0 \\
0 \\
0 \\
0 \\
0 \\
0 \\
4 \\
0 \\
7 \\
0 \\
\pm \\
0 \\
0 \\
0 \\
0 \\
0 \\
5 \\
0 \\
0 \\
0 \\
0 \\
0 \\
0 \\
0\end{array}$ & 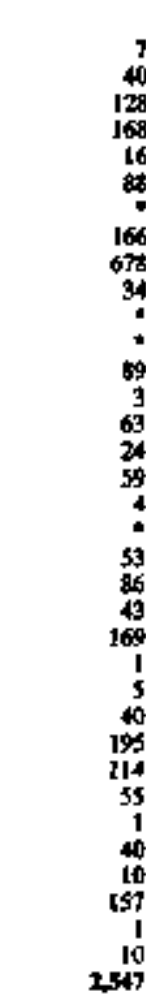 \\
\hline 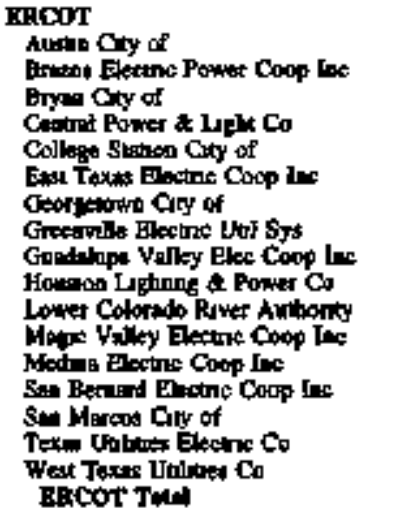 & $\begin{array}{r}30 \\
3 \\
19 \\
59 \\
0 \\
0 \\
0 \\
0 \\
100 \\
88 \\
2 \\
0 \\
5 \\
3 \\
968 \\
10 \\
1,51\end{array}$ & $\begin{array}{r}0 \\
0 \\
10 \\
0 \\
0 \\
0 \\
1 \\
0 \\
7 \\
0 \\
0 \\
1 \\
0 \\
2 \\
0 \\
0 \\
0 \\
77\end{array}$ & $\begin{array}{c}0 \\
0 \\
0 \\
0 \\
0 \\
0 \\
0 \\
\mathbf{J} \\
\mathbf{6 0} \\
0 \\
20 \\
0 \\
0 \\
4 \\
0 \\
0 \\
0 \\
01\end{array}$ & $\begin{array}{r}0 \\
0 \\
0 \\
0 \\
1 \\
* \\
* \\
0 \\
6 \\
0 \\
0 \\
0 \\
8 \\
0 \\
0 \\
29 \\
0 \\
0\end{array}$ & $\begin{array}{l}3 \\
0 \\
0 \\
0 \\
0 \\
0 \\
0 \\
1 \\
0 \\
0 \\
0 \\
0 \\
0 \\
0 \\
0 \\
0 \\
0 \\
4\end{array}$ & $\begin{array}{r}373 \\
7 \\
29 \\
99 \\
1 \\
1 \\
2 \\
73 \\
100 \\
115 \\
9 \\
8 \\
6 \\
3 \\
1.262 \\
10 \\
248\end{array}$ \\
\hline 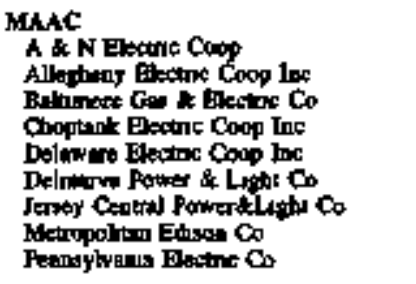 & $\begin{array}{r}0 \\
129 \\
0 \\
0 \\
36 \\
36 \\
35 \\
65\end{array}$ & $\begin{array}{r}1 \\
40 \\
0 \\
3 \\
11 \\
0 \\
34 \\
0 \\
0\end{array}$ & $\begin{array}{r}0 \\
11 \\
0 \\
0 \\
0 \\
0 \\
0 \\
0 \\
0\end{array}$ & $\begin{array}{r}0 \\
5 \\
74 \\
5 \\
0 \\
0 \\
0 \\
185 \\
3\end{array}$ & $\begin{array}{l}1 \\
1 \\
0 \\
0 \\
0 \\
0 \\
0 \\
0 \\
0 \\
0\end{array}$ & $\begin{array}{r}2 \\
70 \\
200 \\
8 \\
11 \\
360 \\
150 \\
200 \\
69\end{array}$ \\
\hline
\end{tabular}

See thinteoks it tod of table 
Table 19. U.S. Electrle Utillty Actual Peak Load Redhetkons by North American Blectric Relibabitity Council Region mat Howit by DSM Proprom Category, $19 \%$ (Megawatts) (Continued)

\begin{tabular}{|c|c|c|c|c|c|c|}
\hline 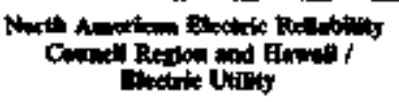 & Eimengy & Direct Lend & Liempiptihte & Dher kend & 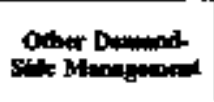 & 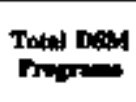 \\
\hline
\end{tabular}

Mucc (Comthand)

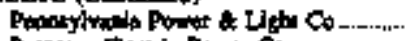
Powne Eectric Ponte Co.

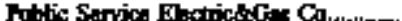

Souther Merylned Et Coop lne

Mhat Tot

$\begin{array}{rr}30 & 0 \\ 266 & 0 \\ 311 & 107 \\ 8 & 34 \\ 36 & 20\end{array}$

$\underset{0}{9}$

0
154
0
0
404

0
0
0
15

30

4

167

40.

514

1,7

\section{MAIT}

Booss Electit Toop.

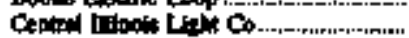

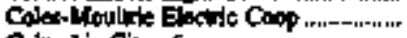

Columiti Cits of...

Commonenth Fo"son Ob

Com

Cure Rumb Electrle Coip los...........

Rater ill Eloctrie Coop

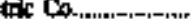

Mrétionoc Poble Utilitie

Mishifinld Cty of

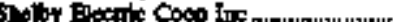

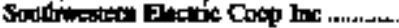

Sprigateld City of........._-_._._._......

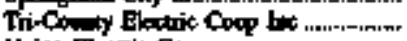

Unich Dexis Co..

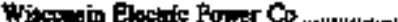

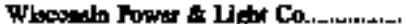

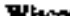

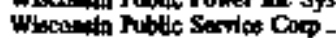

MAIN Tw

0
0
0
3
18
0
1
2
47
3
1
0
0
8
0
7
377
79
79
172
97

0
116
7
4
150
5
3
4
19
0
0
10
11
0
11
125
326
0
0
0
75

0
0
0
0
51
0
0
0
0
0
0
0
5
0
0
0
10
0
0
18
8

$\begin{array}{rr}0 & 3 \\ 0 & 115 \\ 0 & 10 \\ 0 & 12 \\ 0 & 234 \\ 12 & 17 \\ 0 & 7 \\ 0 & 11 \\ 0 & 66 \\ 0 & 3 \\ 0 & 1 \\ 0 & 11 \\ 0 & 21 \\ 0 & 8 \\ 0 & 11 \\ 0 & 134 \\ 0 & 663 \\ 0 & 79 \\ 0 & 29 \\ 0 & 190 \\ 12 & 1925\end{array}$

MAPTCL:

Arous Cliy of

Anota Cliy of

Alostia Cir of...

Butcon Destrie Coop.

Canital Electir Coop Inc...

Evinte Coop Int.--....

Codw FaT Cin of

Centid low Powit Coopt...................

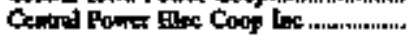

Curb Cly of

Clent Blectric Coop

Coop Paner ket .

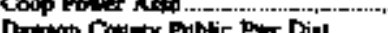

Defison Gy of ..................................

Full Grand Fontr Gity of

Bud River fios Power Coop lax.

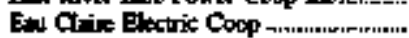

Friphonl Pulis Utitices Comn...

Focboro-Mownt bectic Coop .

Grin-Lainyelin Blecric Cosp

In:ming Pone Co

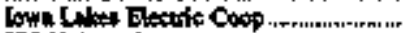

ies Uititia lec..

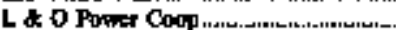

Leringion Ciry of ....

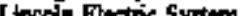

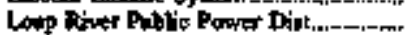

Mrthall City of

MCo"ned Power Coop

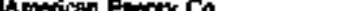

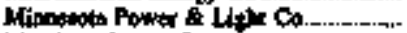

Minolote Powe Cocp bic.

Mocmibend Ciky of ....

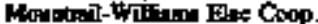

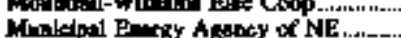

See footwotes at and of toble.

\begin{tabular}{|c|c|c|c|c|c|}
\hline 0 & I & 0 & 0 & $\begin{array}{l}0 \\
0\end{array}$ & I \\
\hline 1 & I & 1 & 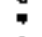 & 0 & 3 \\
\hline - & 4 & 0 & 0 & 0 & 4 \\
\hline 0 & 2 & 0 & 0 & 0 & 2 \\
\hline$\star$ & 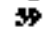 & 5 & 0 & 0 & $\mathbf{4}$ \\
\hline • & 0 & 0 & 0 & 0 & $\bullet$ \\
\hline • & $\theta$ & 0 & 0 & 0 & $\bullet$ \\
\hline 0 & 15 & B & $\mathbf{0}$ & 0 & 15 \\
\hline D & I & " & $\mathbf{1}$ & 0 & 2 \\
\hline$\bullet$ & 3 & 6 & 0 & $\boldsymbol{0}$ & 3 \\
\hline 9 & 0 & 0 & 0 & 0 & 9 \\
\hline 0 & 0 & $\star$ & 0 & 0 & * \\
\hline 0 & 2 & 9 & 0 & 0 & 2 \\
\hline 0 & $\mathbf{I}$ & 0 & (6) & 0 & 1 \\
\hline 0 & 50 & 0 & 0 & $\mathbf{0}$ & 53 \\
\hline t & 4 & 0 & 0 & 0 & 4 \\
\hline 0 & 2 & " & 0 & I & 3 \\
\hline 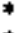 & 3 & I & $\mathbf{0}$ & 0 & 4 \\
\hline+ & 5 & 0 & 0 & 0 & s \\
\hline 26 & 0 & 0 & 0 & 0 & 26 \\
\hline 6 & $\mathbf{0}$ & I & 2 & 6 & 3 \\
\hline 32 & 17 & tr & 96 & 0 & 145 \\
\hline D & 2 & 0 & 0 & 0 & $\mathbf{z}$ \\
\hline 0 & 1 & 0 & 0 & 0 & I \\
\hline 3 & 0 & 4 & 1 & 0 & 4 \\
\hline $\mathbf{0}$ & 0 & 6 & 0 & 0 & 6 \\
\hline 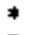 & ! & I & 0 & 0 & 3 \\
\hline 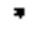 & 0 & 0 & 0 & 0 & 4 \\
\hline 94 & 38 & 165 & 0 & 2 & 300 \\
\hline 28 & 15 & 200 & 0 & 0 & 243 \\
\hline 0 & 300 & 0 & 0 & 0 & 360 \\
\hline 1 & 11 & 2 & 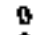 & 7 & 13 \\
\hline$?$ & 1 & 0 & 0 & 0 & 4 \\
\hline 5 & 18 & I & 2 & 0 & 25 \\
\hline
\end{tabular}


Table 19. U.S. Electric Utility Actunl Peaic Laed Reductions by North Anaerican Electric

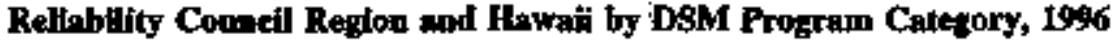
(Megawatts) (Continued)

\begin{tabular}{|c|c|c|c|c|c|c|}
\hline 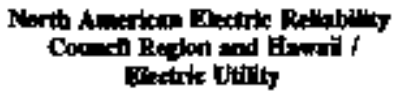 & 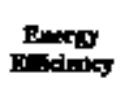 & $\begin{array}{l}\text { Dhed Loed } \\
\text { chetind }\end{array}$ & Intriple & 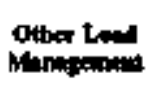 & 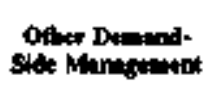 & 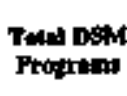 \\
\hline
\end{tabular}

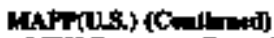

Mid Rapoutas Grorp he

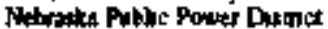

Nod:k Ploctuc Coop inc

Nonthor Seres Popre Co of MIN

Nortem Sterg Power Co of W

Nonthores kopp Nowrer Coop

Northosestan Publa Service Ca

Nosthweotion Wuconup Bles Co

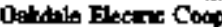

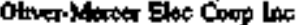

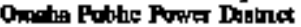

Ower Tel Purre Co

Gracosini City of

People 1 Coop Row hasp

peetre ciny of

Potk-Barad Ebatic Coop

R \& R Eloctanc Coop in:

Ruct Latse Unilroes

Rocheste Poblic Uollte:

Rontin Eexte Coop late

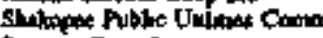

Silengror Oty

5rpener Whar Legrep Pouner Co

Thef Rurt Fids Ciry of

Trempersed Electic Coxp

Tn-Coniny Bustene Coop

Untud Power Alo

Veredrye Ekstic Coop In

Venow Iectic Coop

Yart Cornty Runl Pib Por Das

MArE(US, Twa:

NICE(U)

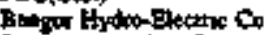

Blechuome Valky Blectinc Co

Bnoton Edsum Co

Brintre Towe of

Burlingtap Cry of

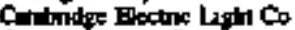

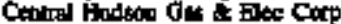

Oetrat Mane Powrer Co

Cental Vermoal Ph \$or Corp

Chropes Cyy of

Cinzent loilutes Co

Conmomeallt Electinc Co

Conconts Elextre Co

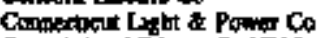

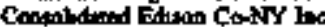

Equter Bdroos Co

Buter a Hulptet Eectic Co

Fithber Ges : Eloc lugt Co

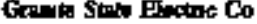

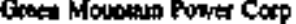

Hingend Cxy of

Holyole Cly of

kamplawn Oily of

Long lyand Lughing Co

Matue Miblue Service Co

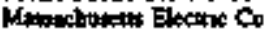

Mnoves Tow of

Nermpinate Elocine Co

Now England Forome Co

Now Hrmphin Blac Coos he

Nor York san Eloc \& do Con

Atewport Eectioc Conp

Nupon Molnow Pinwa Corp
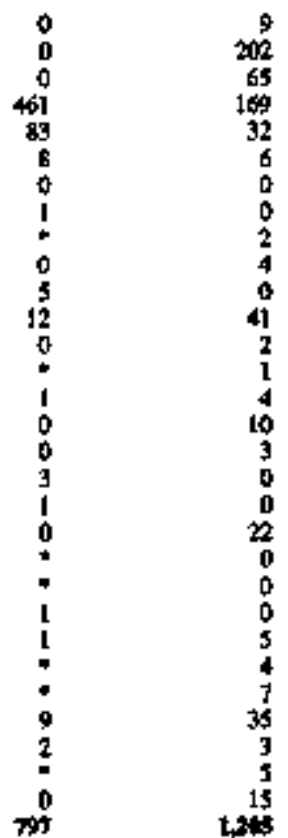

0
9
0
384
60
0
0
0
0
0
0
4
6
0
0
0
0
0
0
0
0
0
0
1
0
0
0
0
0
0
0
0

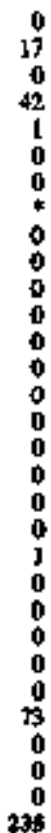

0
0
0
0
13
0
0
0
0
0
0
0
0
0
0
0
0
0
0
0
0
0
0
0
0
0
0
0
0
0
15

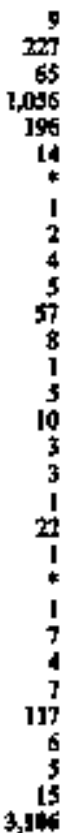

Set footnotes at end of teble

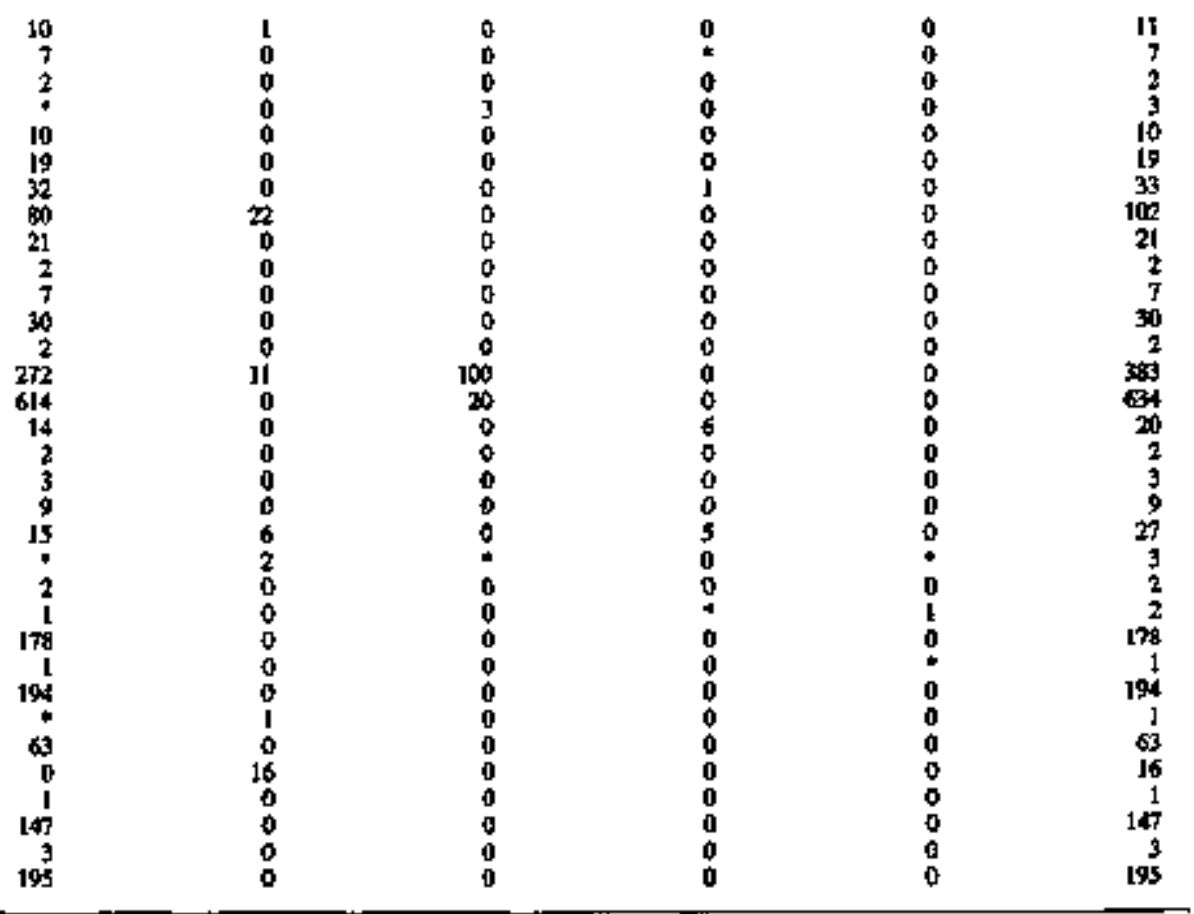


Table 19. U.S. Electric Utiltb Actal Ptak Land Reduetions by North Aneriegn Electrle Reliability Council Rejion and Hawalt by DSM Program Catezory, 19\%6 (Megawetts) (Contimued)

\begin{tabular}{|c|c|c|c|c|c|c|}
\hline 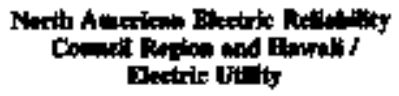 & Enking & $\begin{array}{l}\text { Dired Land } \\
\text { Controd }\end{array}$ & Itempingle & 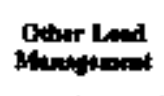 & 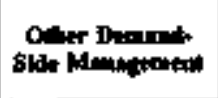 & $\begin{array}{l}\text { Thal DSM } \\
\text { Progtintal }\end{array}$ \\
\hline
\end{tabular}

NPCCDSS (C)minim)

Hocinod Cxy of n.

Oryprs the

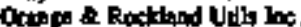

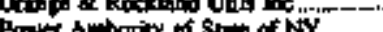

Pahlic Strixe Co of $\mathbf{K}$ 나

Reniting Tows of

Thente of of

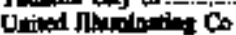

Vertood Eletrife Coep f

Welloter Tont of

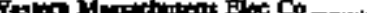

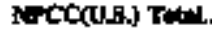

stac

Ailsed Enctric Cocp he

Alabaren Electric Cocp lne-..._..........

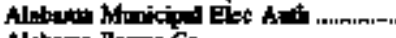

Alatora Power Co

Alternole Cln of

Alumine Electric Mlenber Corp..........

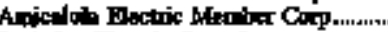

Befielly Bectic Coap lis

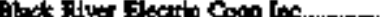

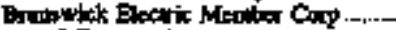

BARC Elotic Coop Int

Cancel Cty of

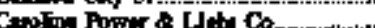

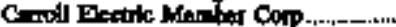

Genoll Gowtia E Mewht Cop .......

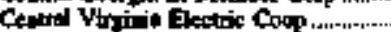

Choctunt the Eloc Coop Ins.

Cles Elactine Caco lnt

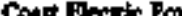

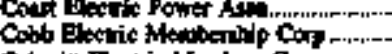

Cotofidt Fintric Manbers Corp............

Conaricy Eloctie Cor

Conction

Dothen Coty of

Dorizins City of

Barlor Comber utity syom

Extr Potst Cly of

Finfletd Eloctic Coop In

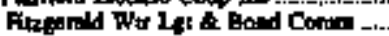

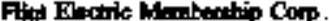

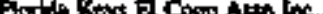

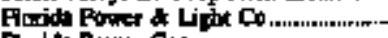

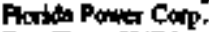

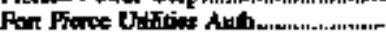

Clatifiney Ciry of

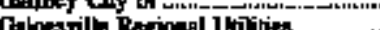

Gowitin Pown Co

Gindy Conky blos Mertiker Conp.....-

Creserntle Utilities Corm.

frees Com of Pulle wrots

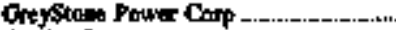

ciifon City of .

Calf Powits Co....

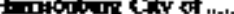

Hot byestic Menter Cop

Haymood Dinctric Momber Corp.........

trigh Point Trom of

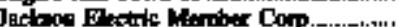

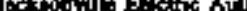

$\begin{array}{rr}2 & 0 \\ 74 & 0 \\ 65 & 0 \\ 3 & 0 \\ 37 & 0 \\ 1 & 0 \\ 3 & 0 \\ 56 & 2 \\ 0 & 0 \\ 2,219 & 8 \\ 43 & 0\end{array}$

0
0
61
0
4
6
0
0
71
0
0
15
200

0
0
0
0
0
0
0
1
0
4
0
0
0
10

9
134
6
7
37
3
3
90
1
7
64
254

Sec foctuoted and of toble.

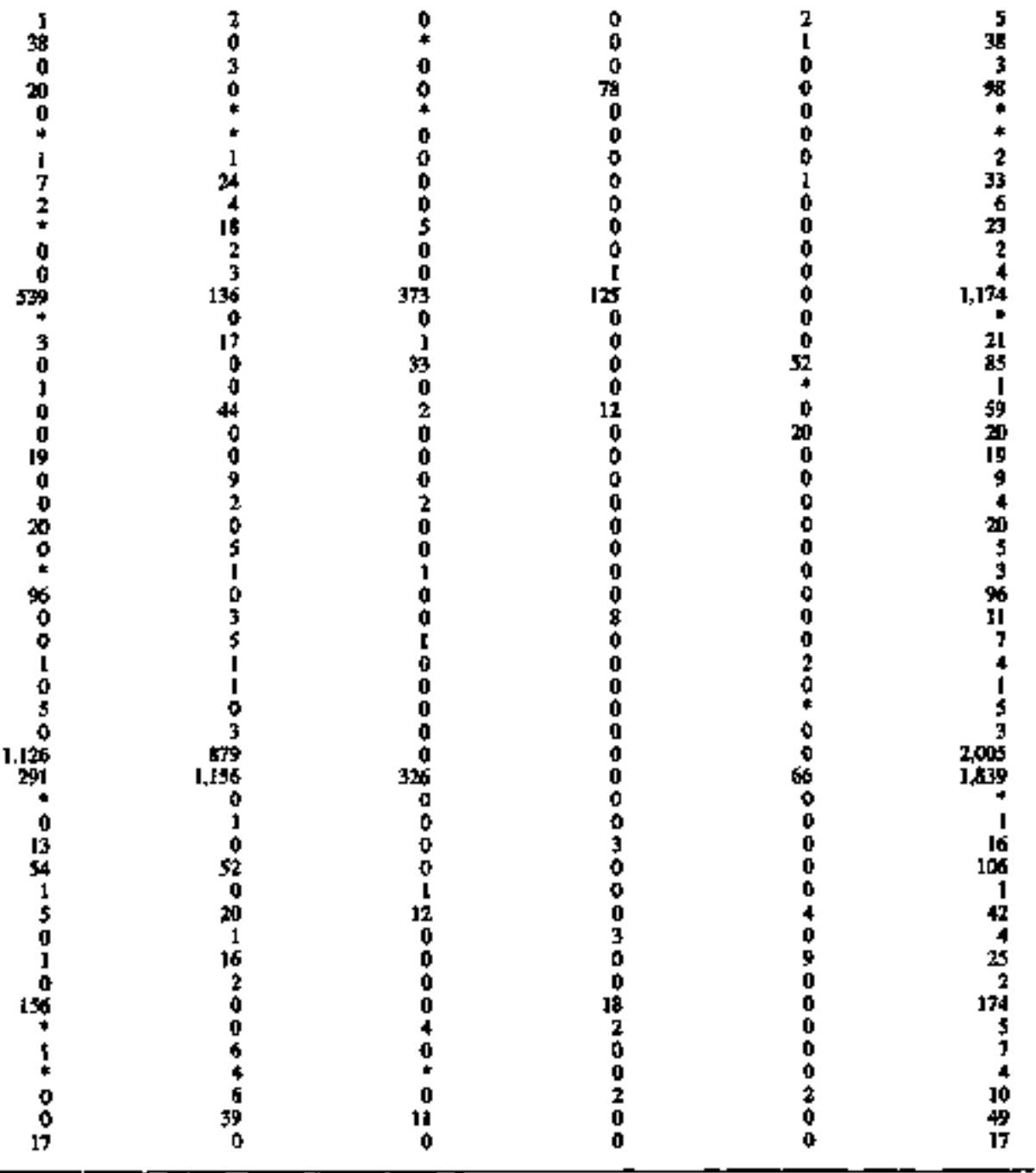


Table 19. U.S. Electric Utility Actual Peet Loed Redactions by North Anerican Electric Reliability Comact Rejion and Hawait by DSM Program Category, 1996 (Megawatts) (Continued)

\begin{tabular}{|c|c|c|c|c|c|c|}
\hline 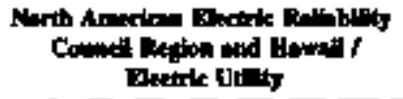 & Dotidy & $\begin{array}{l}\text { Dintat latal } \\
\text { Control }\end{array}$ & 10terrumithe & 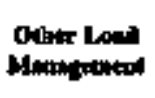 & 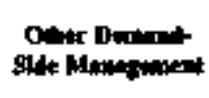 & 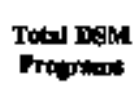 \\
\hline
\end{tabular}

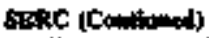

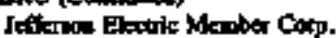

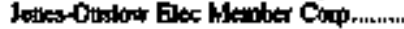

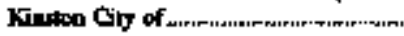

Kitsinger Uility Alotity.

1.tolete cin of

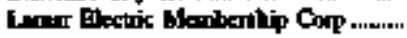

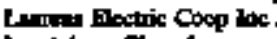

Lutridet Ory of

I

Ler Cowny Eloctic Conp lits

Lenting City of..

Lomberion Cily of

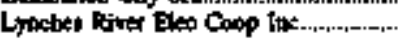

Nineses Cry of.

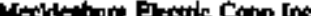

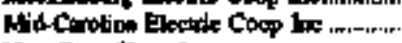

Net Bent City of

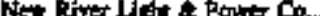

Now Snypat Beth Ukt Comp......

Newbercy City ot

Nooth Crodino Bowton M A

North Caroline \#d Mentiber Corp.u........

Narth Crodion then Pomer Agay -..-

Noethre Nack bec Coop ho

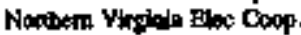

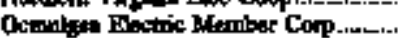

Oningtors City of

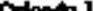

Fireto Fectic Cocp hac ...................."

Pee Dee Ploxic Coop toc.

Pince Beroge Eletric Coop

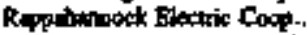

Rurle Elextrix Memberdip Coos .......

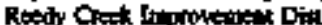

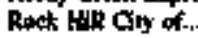

Rocky Hour City of

Sutill Rinn Bee Nember Cotp

Sivrand Enctic \& Potar Co

Smexe Eextric Mented Cofp

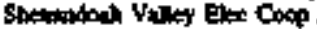

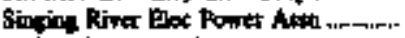

Sol thifievd Tom of

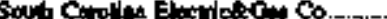

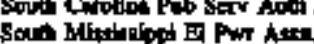

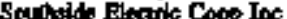

5 atre Fingtic Cop

Friloweses Ciy of.

Tution Eloctic Co

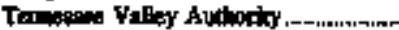

Thomenow ofy of ...............................

Tidend Eectic Momicer Corp _........

Tri-Comby Ele Bembet Cop

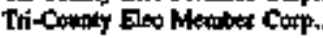

Trop Exetric Member Cop

Unica Oly of

Wirtinda Boctries \& Powrer Co.

Warting boe City of

Wilson city of

Withrooches Biver Dec Coop.

Yoft Electrit Coop Iec

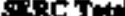

Sec fortosed in ood of toble.

\begin{tabular}{|c|c|c|c|c|c|}
\hline $\begin{array}{l}1 \\
2\end{array}$ & $\begin{array}{l}9 \\
8\end{array}$ & $\begin{array}{l}3 \\
3\end{array}$ & $\begin{array}{l}0 \\
0\end{array}$ & $\begin{array}{l}0 \\
0\end{array}$ & $\begin{array}{l}12 \\
12\end{array}$ \\
\hline$\theta$ & 2 & 15 & 4 & 0 & 30 \\
\hline 3 & 0 & 0 & 0 & 0 & 3 \\
\hline 1 & As & 0 & 0 & 0 & 4 \\
\hline D & 0 & 0 & I & 0 & 1 \\
\hline - & 0 & $\theta$ & $*$ & 0 & $*$ \\
\hline 0 & 3 & 0 & 0 & 0) & 3 \\
\hline 0 & 4 & 0 & i & 0 & 4 \\
\hline 6 & 56 & 6 & 0 & 0 & 58 \\
\hline 0 & 3 & 0 & 5 & 3 & 11 \\
\hline ) & 2 & 0 & 0 & 0 & 2 \\
\hline$i$ & 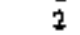 & 0 & 0 & 2 & 4 \\
\hline 0 & 2] & 0 & 0 & 0 & 21 \\
\hline 0 & 6 & j & 3 & 3 & 14 \\
\hline 3 & 4 & 4 & 0 & 3 & 10 \\
\hline 0 & 6 & 0 & 4 & a & 10 \\
\hline 0 & * & 0 & 0 & 0 & * \\
\hline 0 & 8 & 0 & 0 & 0 & 8 \\
\hline 0 & 1 & 0 & 0 & 0 & 1 \\
\hline 0 & 53 & IS & 89 & 23 & $1 \pm 0$ \\
\hline 0 & 168 & 88 & 0 & 0 & 256 \\
\hline 0 & 22 & 7 & 32 & 0 & b] \\
\hline 0 & $\mathbf{3}$ & 0 & 0 & 0 & 3 \\
\hline 1 & 29 & 3 & 0 & 0 & 32 \\
\hline 0 & I & 0 & 0 & 0 & ] \\
\hline 0 & 0 & 2 & 2 & 2 & 6 \\
\hline 35 & 0 & 2 & 0 & 0 & 37 \\
\hline 1 & 7 & 4 & 3 & 0 & 15 \\
\hline 1 & 2 & 0 & 0 & $*$ & 1 \\
\hline 0 & 14 & 0 & 0 & 0 & 14 \\
\hline 0 & 27 & 6 & 0 & 0 & 33 \\
\hline$\bullet$ & I & 1 & 0 & 0 & 2 \\
\hline I & 0 & 0 & 0 & 0 & 1 \\
\hline 0 & 3 & 0 & 0 & 4 & 7 \\
\hline 0 & 10 & 0 & 8 & 8 & 25 \\
\hline I & 8 & 0 & 0 & 0 & 9 \\
\hline 1 & 0 & 0 & 0 & a & 2 \\
\hline • & 19 & 0 & I & 0 & 20 \\
\hline 0 & II & 3 & 0 & 0 & 13 \\
\hline 4 & 0 & 0 & 2 & 0 & 7 \\
\hline 0 & 2 & a & 0 & 0 & 2 \\
\hline 106 & 0 & 0 & 0 & 0 & 106 \\
\hline 35 & 16 & o & 0 & 0 & 51 \\
\hline 6 & 0 & 5 & 37 & 0 & 48 \\
\hline 0 & 6 & 5 & 3 & 0 & 14 \\
\hline 3 & 35 & I0 & 0 & 0 & 52 \\
\hline 21 & 0 & 0 & 0 & 5 & 36 \\
\hline 242 & 42 & 0 & 0 & 0 & 284 \\
\hline 40 & 58 & 1.800 & 0 & 0 & 2,338 \\
\hline 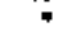 & 4 & 0 & . & 0 & 5 \\
\hline 0 & 8 & 4 & a & 0 & 12 \\
\hline 0 & 0 & 0 & . & 0 & $*$ \\
\hline 0 & 3 & + & 0 & 0 & 3 \\
\hline 0 & 8 & 0 & 0 & 0 & B \\
\hline 0 & I & 0 & 0 & 0 & I \\
\hline 9$]$ & it & 12 & 2 & 0 & $10 x$ \\
\hline 0 & 11 & a & 0 & 0 & II \\
\hline 0 & 10 & 19 & 18 & 0 & 46 \\
\hline 0 & 0 & 0 & 4) & 0 & d) \\
\hline 1 & 0 & 10 & 0 & 0 & 11 \\
\hline 3,405 & 3,231 & 2,793 & 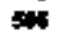 & 212 & 10,200 \\
\hline
\end{tabular}


Table 19. U.S. Electric Utility Actual Penk Lood Reductions by North American Electric Reliahility Coucil Region and Bowall by DSM Program Catezory, 19\$6 (Megawatts) (Continued)

\begin{tabular}{|c|c|c|c|c|c|c|}
\hline 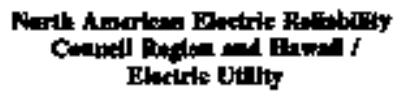 & Enmov' & $\begin{array}{l}\text { Birted Land } \\
\text { enintol }\end{array}$ & $\begin{array}{l}\text { Inaterioptbls } \\
\text { Loth }\end{array}$ & 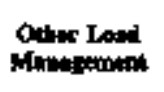 & 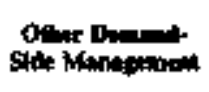 & Thet DET \\
\hline
\end{tabular}

Bif $P$

Alfalfa Eloctine Coop In

Nhan Dry of

C \& L Electre Coop Con

Cerito Blactas Coop In

Campl Elocist Coop Cor

Cetni Roral Eloctrix Coct

Cookmon thith Bloc Coxp toc

Orophead Blecore CoOp Cor

Detto Electinc Rinut Anth

Dixte Electre Membetho Cop

Dances Oty of

Empere Divion Electric Co

Finnotir Electro Coop iec

Fun Bestix Coop tonp

Oront Electric Conp In

Idipedand Oty of

Endur Electine Coop loc

Kmag Oty Cry of

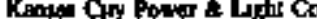

Kutses Elvenc Powar foop in

Kona Cu a Elonic Co

Mrsurpop Ony Elex Copp In

Nonit Hatouts Elec Coog I-

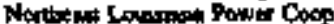

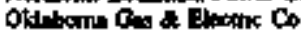

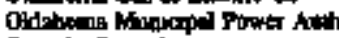

Ostedia City of

Onatk Elactot Coog he

Petu ke Eloctic Coup Corp

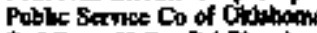

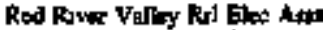

South Cental Ad e Coop Ino

South Protan Blectinc Crop Ins

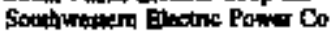

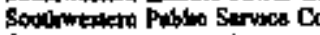

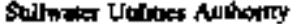

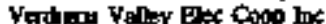

Weator Resoumces Inc

Who Rusar Vollay 向 Coop lac

Woodniti Electnk Coop Corp

SPT Ther

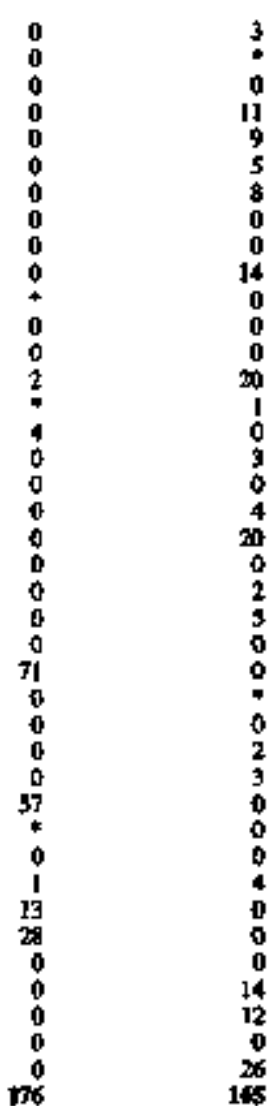

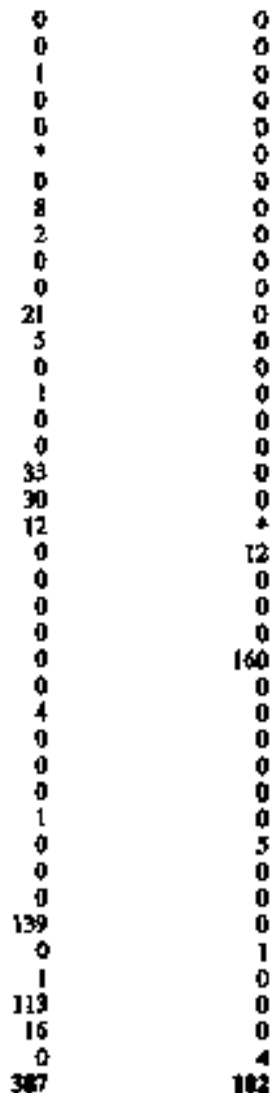

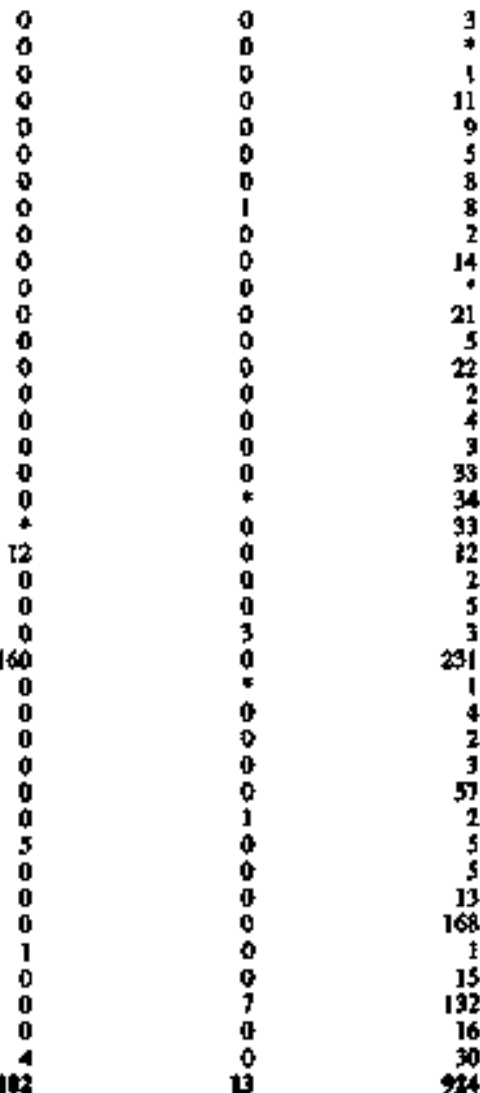

wscc(Us)

Alingdt City of

Antom Gyy of

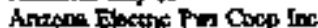

Aratim Puble Serves Co

Sowlder Cry city of

Bannifil Ciry Cty of

Colvedo Spning Gly of

Dire Bucatante R E A fot

El Paso Elowerc Co

Engan Cxy of

Fant Collos City of

Holt Crons Electic Atst h.

Impend lenprod Damp

1. Ple Bectec And toc

Longend City of

Lex Ancelat Ory of

Lowiland Cary of

Modetta laybon Dienç

Mohrire Foctine Coop lec

Monlong Powar Co

Monitum Party Electinc Inc

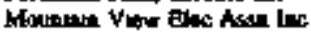

Nurepactic Eleconc Coop he

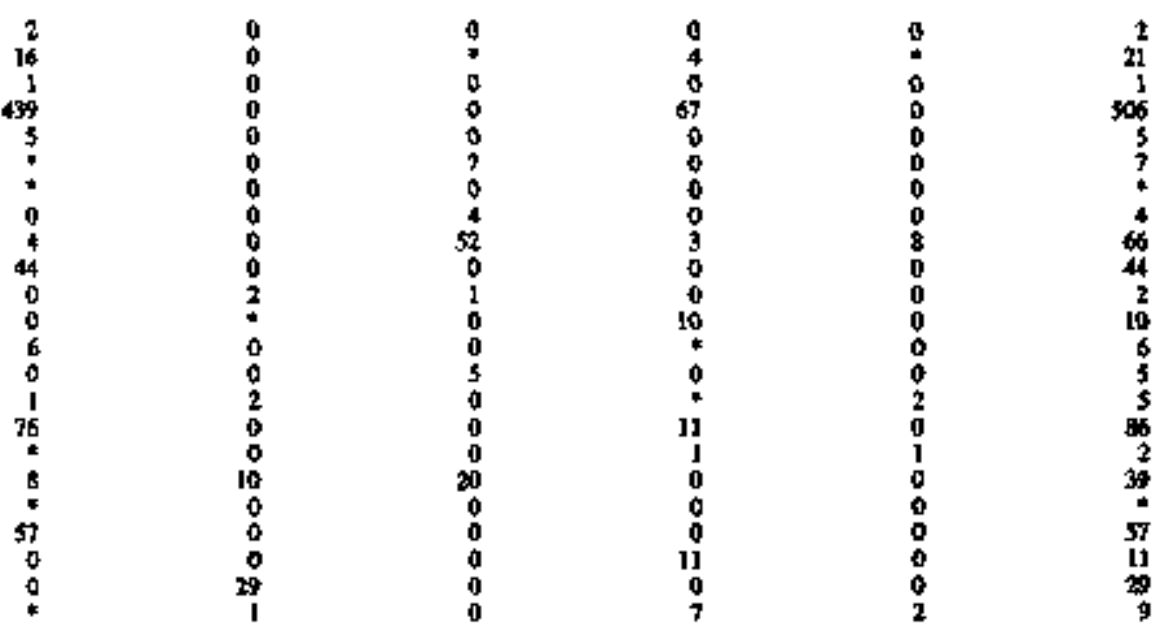

fect foobiotes at end of ublo 
Table 19. U.S. Dlectric Uthity Acturil Peak Load Reductions by North American Electric Reltabilky Conacll Region and Hawai by DSM Propram Catesory, 19\%

(Megswasts) (Continuad)

\begin{tabular}{|c|c|c|c|c|c|c|}
\hline 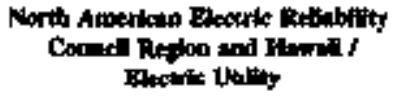 & $\begin{array}{l}\text { Enetis } \\
\text { Denciency }\end{array}$ & $\begin{array}{l}\text { bired Led } \\
\text { Centrits }\end{array}$ & $\begin{array}{c}\text { tomartaptule } \\
\text { late }\end{array}$ & Mthey Lowim & 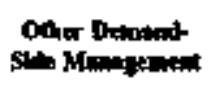 & 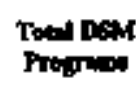 \\
\hline
\end{tabular}

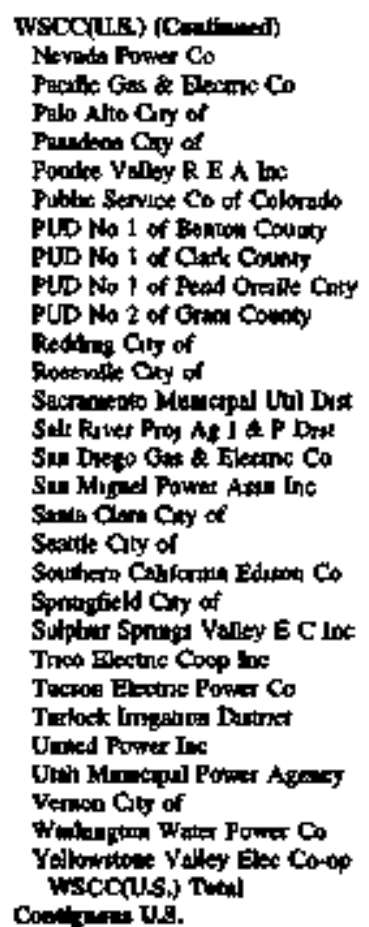

AscC

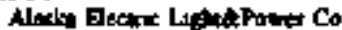
Goldes Valloy Flac Ases lan Asce Twal

33
614
6
5
1
66
1
9
1
17
25
3
139
86
196
0
0
60
1.46
4
0
0
32
2
4
1
0
90
0
3,517
14,233

0
505
0
0
0
232
0
0
0
0
2
0
60
0
41
0
7
0
0
0
0
1
6
0
3
0
0
0
0
96
$7 \% 7$

33

$1,1] 9$

6

I

29

1

62

30

429

136

24

7

60

1,614

4

38

2

1

8

1

S,134

2,80

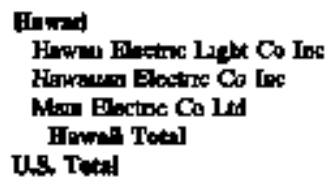

$\begin{array}{ll}0 & 3 \\ 2 & 0 \\ 2 & 3\end{array}$

3
0

0

- Valos late ter 0.5

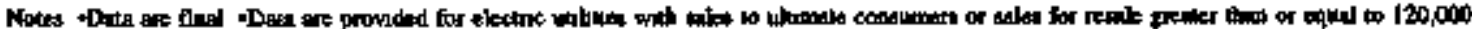

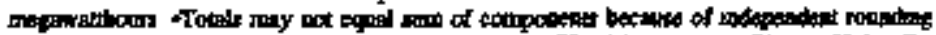

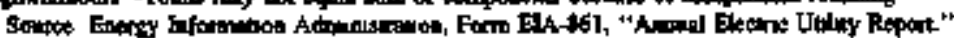


Toble 20. U.S. Electric Utility Actual Peak Load Reductions by North American Electric Reliabitity Comeil Region and Hownit by Cless of Ownersilp and Sector, 1996 (Megawatts)

\begin{tabular}{|c|c|c|c|c|c|c|}
\hline 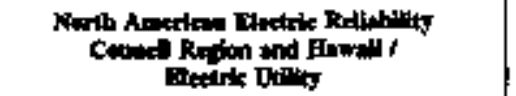 & 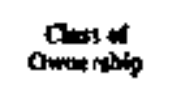 & 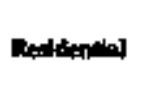 & Cocaneretal & Indadrtal & Odner & Totid \\
\hline 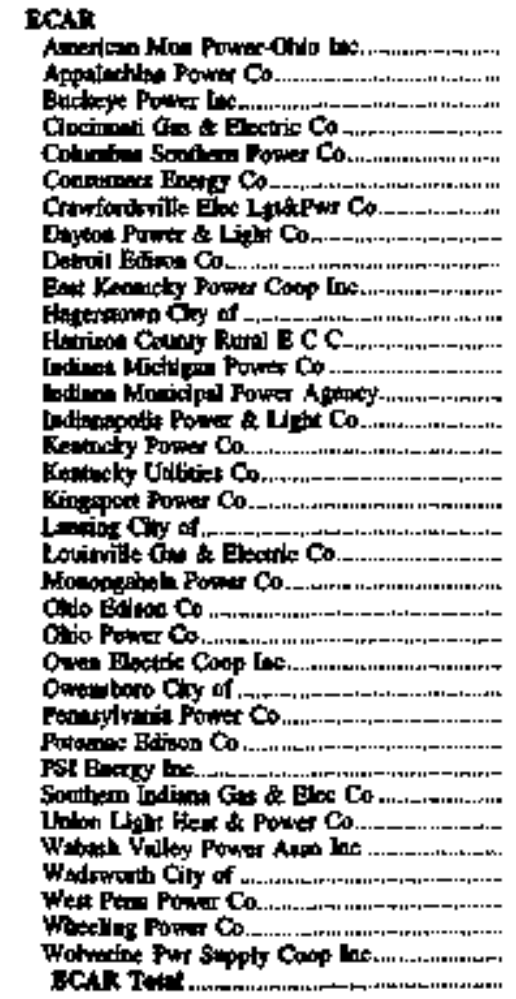 & 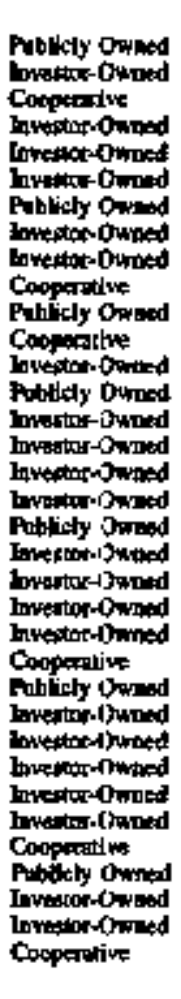 & $\begin{array}{r}0 \\
37 \\
103 \\
26 \\
12 \\
11 \\
0 \\
17 \\
162 \\
34 \\
4 \\
4 \\
7 \\
3 \\
4 \\
12 \\
17 \\
4 \\
0 \\
4 \\
26 \\
15 \\
17 \\
1 \\
0 \\
0 \\
84 \\
20 \\
33 \\
1 \\
40 \\
0 \\
16 \\
1 \\
10 \\
711\end{array}$ & $\begin{array}{r}0 \\
1 \\
0 \\
30 \\
1 \\
21 \\
0 \\
17 \\
13 \\
0 \\
0 \\
0 \\
1 \\
0 \\
17 \\
0 \\
1 \\
0 \\
1 \\
1 \\
33 \\
18 \\
4 \\
0 \\
0 \\
0 \\
45 \\
55 \\
13 \\
0 \\
0 \\
0 \\
26 \\
0 \\
0 \\
294\end{array}$ & $\begin{array}{r}7 \\
1 \\
25 \\
212 \\
3 \\
55 \\
132 \\
504 \\
0 \\
0 \\
0 \\
81 \\
0 \\
42 \\
13 \\
34 \\
0 \\
0 \\
52 \\
28 \\
10 \\
51 \\
0 \\
5 \\
40 \\
87 \\
31 \\
8 \\
0 \\
0 \\
10 \\
116 \\
0 \\
0 \\
59\end{array}$ & $\begin{array}{l}1 \\
0 \\
0 \\
0 \\
0 \\
0 \\
0 \\
0 \\
0 \\
0 \\
0 \\
0 \\
0 \\
0 \\
0 \\
0 \\
7 \\
0 \\
0 \\
0 \\
0 \\
0 \\
0 \\
0 \\
0 \\
0 \\
0 \\
0 \\
0 \\
0 \\
0 \\
0 \\
0 \\
0 \\
0 \\
9\end{array}$ & $\begin{array}{r}7 \\
40 \\
128 \\
168 \\
16 \\
85 \\
166 \\
678 \\
34 \\
4 \\
89 \\
3 \\
63 \\
24 \\
59 \\
4 \\
1 \\
53 \\
86 \\
43 \\
169 \\
1 \\
5 \\
40 \\
195 \\
114 \\
55 \\
1 \\
40 \\
10 \\
157 \\
1 \\
10 \\
2847\end{array}$ \\
\hline 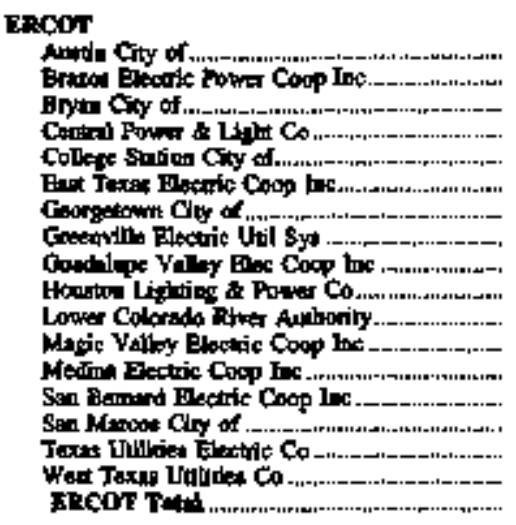 & 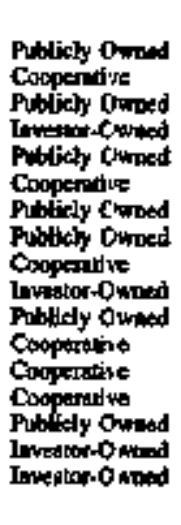 & $\begin{array}{r}204 \\
7 \\
13 \\
36 \\
\vdots \\
1 \\
0 \\
7 \\
64 \\
02 \\
7 \\
0 \\
2 \\
2 \\
555 \\
2 \\
94\end{array}$ & $\begin{array}{r}119 \\
0 \\
1 \\
0 \\
0 \\
0 \\
33 \\
6 \\
1 \\
0 \\
0 \\
1 \\
106 \\
1 \\
0\end{array}$ & $\begin{array}{r}0 \\
0 \\
5 \\
0 \\
0 \\
0 \\
0 \\
2 \\
60 \\
3 \\
26 \\
1 \\
0 \\
4 \\
0 \\
0 \\
0 \\
10\end{array}$ & $\begin{array}{l}0 \\
0 \\
5 \\
0 \\
0 \\
0 \\
+ \\
0 \\
6 \\
0 \\
0 \\
* \\
8 \\
0 \\
0 \\
0 \\
0 \\
19\end{array}$ & $\begin{array}{r}379 \\
7 \\
23 \\
59 \\
1 \\
1 \\
2 \\
73 \\
160 \\
115 \\
9 \\
5 \\
6 \\
3 \\
1,262 \\
10 \\
2,92\end{array}$ \\
\hline 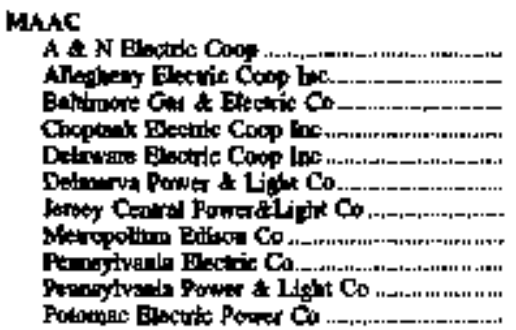 & 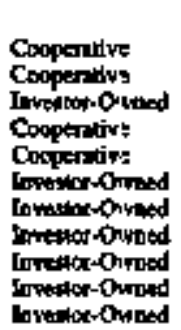 & $\begin{array}{r}2 \\
52 \\
49 \\
3 \\
11 \\
13 \\
54 \\
95 \\
19 \\
15 \\
88\end{array}$ & $\begin{array}{r}0 \\
6 \\
153 \\
0 \\
0 \\
21 \\
96 \\
23 \\
11 \\
10 \\
392\end{array}$ & $\begin{array}{r}0 \\
8 \\
0 \\
5 \\
0 \\
0 \\
0 \\
102 \\
30 \\
4 \\
0\end{array}$ & $\begin{array}{l}0 \\
3 \\
0 \\
0 \\
0 \\
0 \\
0 \\
0 \\
0 \\
1 \\
0\end{array}$ & $\begin{array}{r}2 \\
70 \\
20 \\
8 \\
11 \\
36 \\
130 \\
220 \\
68 \\
30 \\
420\end{array}$ \\
\hline
\end{tabular}

See footooles at end of table. 
Table 20. U.S. Electric Utility Actual Peak Loed Reductions by North Anerican Blectric Reliability Council Reglon and Hawall by Closs of Ownershlp and Sector, 1996 (Mogawatts) (Continued)

\begin{tabular}{|c|c|c|c|c|c|c|}
\hline 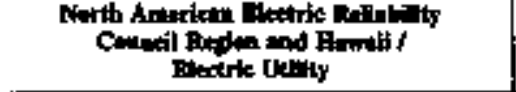 & & Reldroiling & Cor: aref & In lotuten & Other & Totad \\
\hline 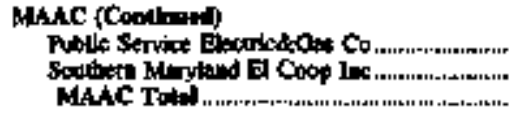 & $\begin{array}{l}\text { Invento-owned } \\
\text { Conpardint }\end{array}$ & $\begin{array}{r}207 \\
4\end{array}$ & $\begin{array}{l}173 \\
877\end{array}$ & $\begin{array}{r}134 \\
0 \\
292\end{array}$ & $\begin{array}{l}0 \\
0 \\
5\end{array}$ & $\begin{array}{r}514 \\
1,72\end{array}$ \\
\hline 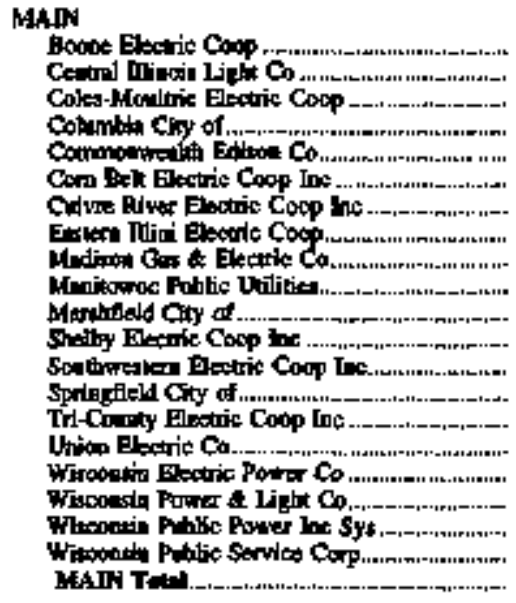 & 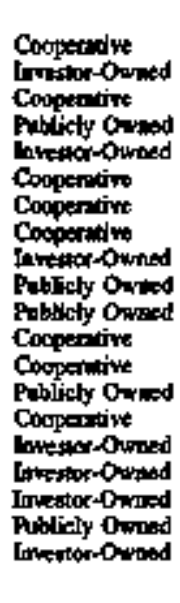 & $\begin{array}{r}3 \\
0 \\
3 \\
5 \\
30 \\
12 \\
3 \\
7 \\
6 \\
1 \\
4 \\
4 \\
9 \\
5 \\
4 \\
4 \\
94 \\
9 \\
3 \\
53 \\
247\end{array}$ & $\begin{array}{r}0 \\
0 \\
0 \\
2 \\
203 \\
5 \\
3 \\
0 \\
57 \\
1 \\
1 \\
6 \\
6 \\
3 \\
7 \\
3 \\
174 \\
64 \\
10 \\
126 \\
67\end{array}$ & $\begin{array}{r}0 \\
116 \\
7 \\
4 \\
1 \\
0 \\
0 \\
4 \\
0 \\
1 \\
7 \\
5 \\
7 \\
0 \\
4 \\
128 \\
395 \\
0 \\
16 \\
0 \\
06\end{array}$ & $\begin{array}{l}0 \\
0 \\
0 \\
0 \\
0 \\
0 \\
1 \\
0 \\
3 \\
0 \\
0 \\
0 \\
0 \\
0 \\
0 \\
0 \\
0 \\
6 \\
0 \\
10 \\
20\end{array}$ & $\begin{array}{r}3 \\
116 \\
10 \\
12 \\
234 \\
17 \\
7 \\
11 \\
66 \\
3 \\
1 \\
11 \\
21 \\
8 \\
11 \\
134 \\
607 \\
79 \\
29 \\
190 \\
1,65\end{array}$ \\
\hline 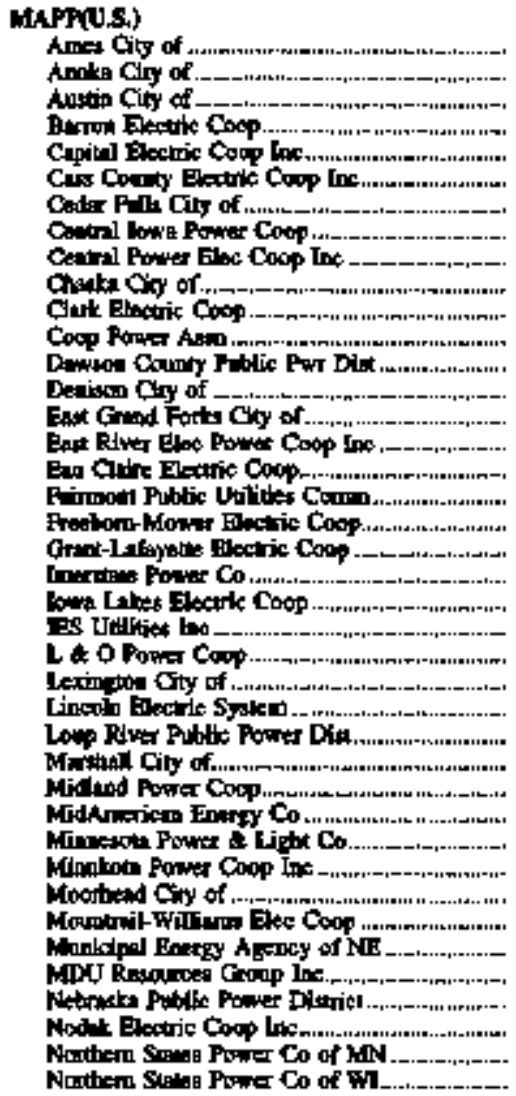 & 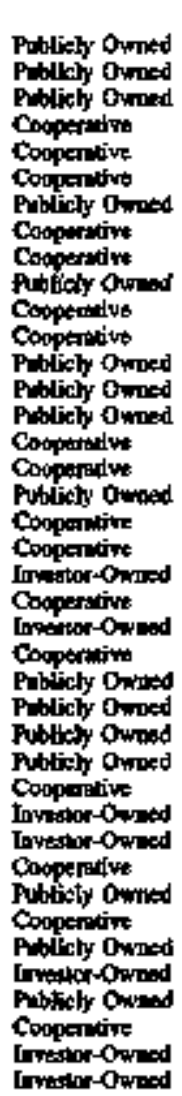 & 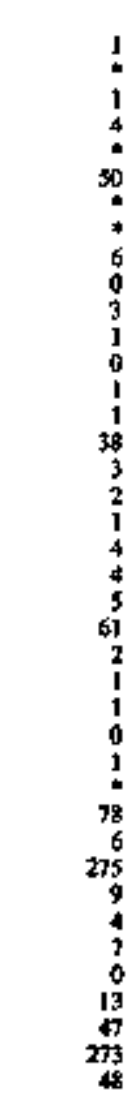 & $\begin{array}{r}0 \\
1 \\
0 \\
2 \\
5 \\
0 \\
7 \\
0 \\
0 \\
0 \\
1 \\
0 \\
0 \\
4 \\
7 \\
0 \\
0 \\
6 \\
1 \\
35 \\
0 \\
0 \\
3 \\
0 \\
1 \\
0 \\
54 \\
11 \\
25 \\
1 \\
0 \\
10 \\
9 \\
0 \\
40 \\
58\end{array}$ & 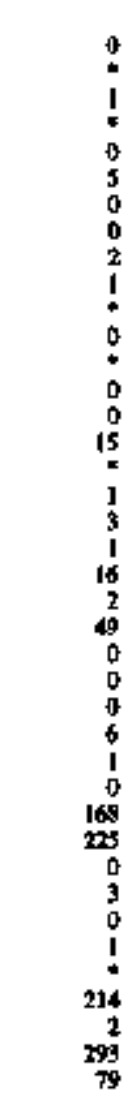 & $\begin{array}{l}0 \\
0 \\
0 \\
0 \\
0 \\
0 \\
0 \\
0 \\
0 \\
2 \\
0 \\
0 \\
0 \\
0 \\
0 \\
0 \\
0 \\
0 \\
0 \\
0 \\
0 \\
0 \\
0 \\
0 \\
0 \\
1 \\
0 \\
0 \\
0 \\
0 \\
0 \\
0 \\
0 \\
0 \\
5 \\
0 \\
0 \\
1 \\
0 \\
1\end{array}$ & 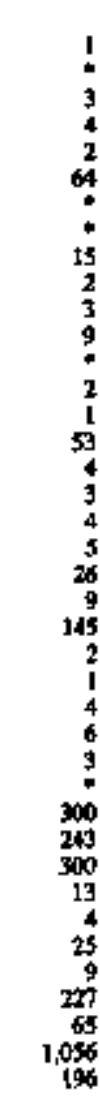 \\
\hline
\end{tabular}

Sec foctions as edd of table. 
Table 20. U.S. Electric Utiliky Actual Peak Lond Reductions by North Ameriean Rlectric Reliability Conancil Ragion and Howait by Class of Owmerstip and Sector, 19\%6 (Megawatts) (Contunued)

\begin{tabular}{|c|c|c|c|c|c|c|}
\hline 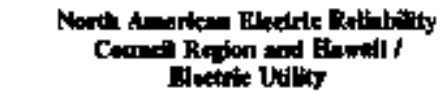 & Oripe of & Destententis & 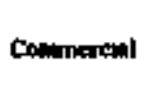 & Indastial & omar & $\mathbf{T}$ it:d \\
\hline 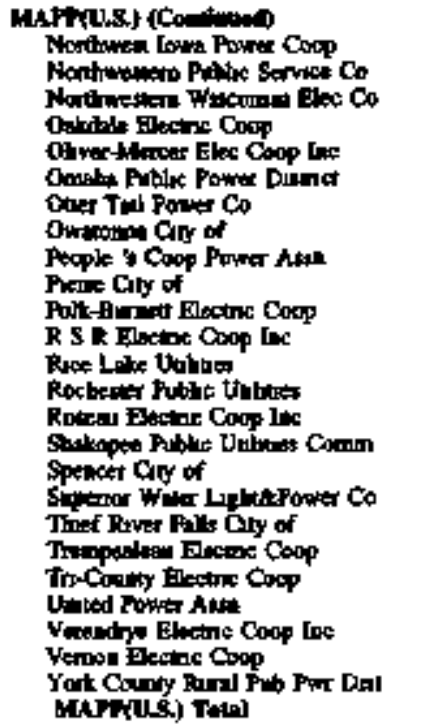 & 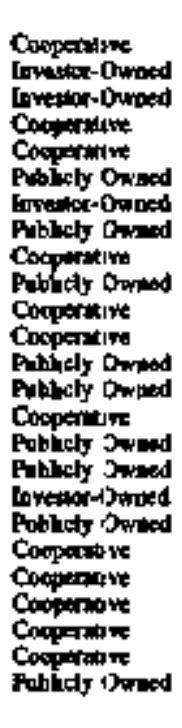 & $\begin{array}{r}14 \\
0 \\
2 \\
2 \\
4 \\
32 \\
2 \\
1 \\
4 \\
10 \\
3 \\
2 \\
7 \\
27 \\
4 \\
7 \\
4 \\
4 \\
112 \\
5 \\
4 \\
1,186\end{array}$ & $\begin{array}{r}* \\
* \\
0 \\
0 \\
1 \\
16 \\
0 \\
0 \\
0 \\
0 \\
1 \\
1 \\
0 \\
1 \\
0 \\
4 \\
3 \\
0 \\
0 \\
1 \\
0 \\
0 \\
786\end{array}$ & $\begin{array}{r}0 \\
0 \\
\mathbf{x} \\
0 \\
0 \\
8 \\
6 \\
0 \\
0 \\
0 \\
0 \\
0 \\
0 \\
0 \\
0 \\
0 \\
0 \\
1 \\
0 \\
0 \\
1 \\
15 \\
120\end{array}$ & $\begin{array}{c}0 \\
0 \\
0 \\
0 \\
2 \\
0 \\
0 \\
0 \\
0 \\
0 \\
0 \\
0 \\
0 \\
0 \\
0 \\
0 \\
0 \\
0 \\
0 \\
0 \\
0 \\
0 \\
0 \\
0 \\
0 \\
15\end{array}$ & $\begin{array}{r}14 \\
1 \\
2 \\
5 \\
51 \\
8 \\
5 \\
5 \\
310 \\
3 \\
1 \\
22 \\
5 \\
1 \\
7 \\
4 \\
717 \\
6 \\
5 \\
15 \\
3,105\end{array}$ \\
\hline 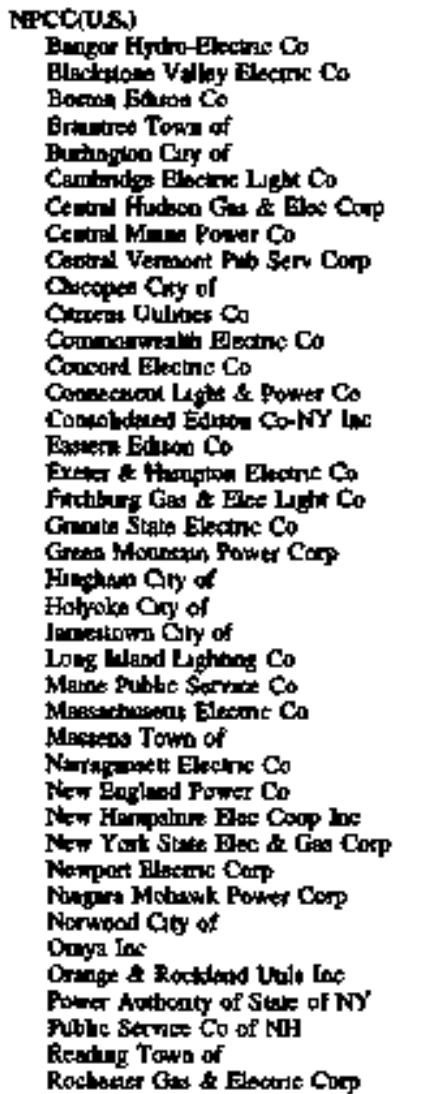 & 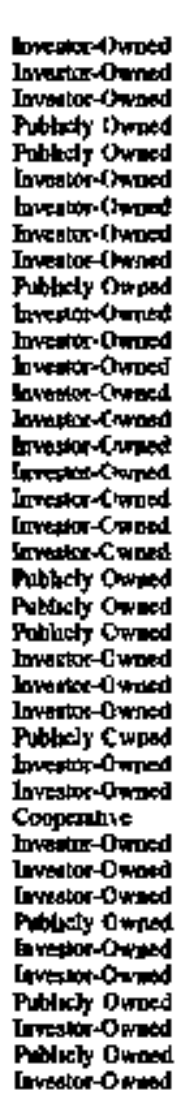 & 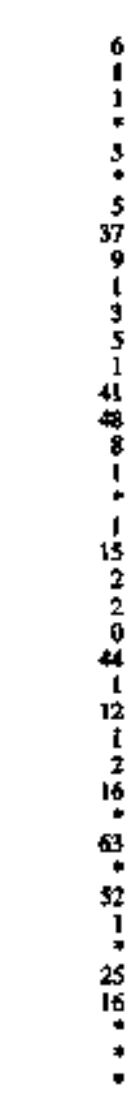 & 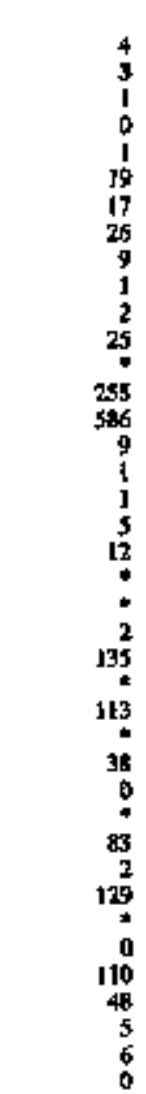 & 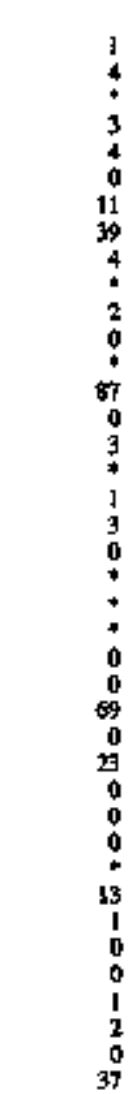 & $\begin{array}{l}0 \\
0 \\
0 \\
0 \\
0 \\
0 \\
0 \\
0 \\
0 \\
0 \\
0 \\
0 \\
0 \\
0 \\
0 \\
0 \\
0 \\
0 \\
0 \\
0 \\
0 \\
0 \\
0\end{array}$ & $\begin{array}{r}11 \\
7 \\
2 \\
3 \\
10 \\
19 \\
33 \\
102 \\
21 \\
2 \\
7 \\
30 \\
2 \\
383 \\
634 \\
20 \\
2 \\
3 \\
9 \\
77 \\
3 \\
2 \\
2 \\
179 \\
1 \\
194 \\
1 \\
63 \\
16 \\
1 \\
147 \\
3 \\
195 \\
9 \\
134 \\
65 \\
7 \\
6 \\
37\end{array}$ \\
\hline
\end{tabular}

Set lootinges to end of buble 
Table 20. U.S. Electric Utility Aches Peak Land Reductions by North American Electric Reliability Concti Region and Fawä̈ by Class of Ownership and Sector, 1996 (Megawatts) (Contunued)

\begin{tabular}{|c|c|c|c|c|c|c|}
\hline 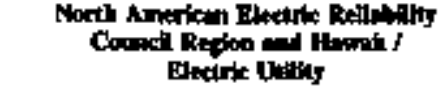 & & 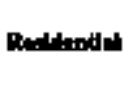 & 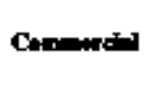 & Industitid & Diter & Tetal \\
\hline 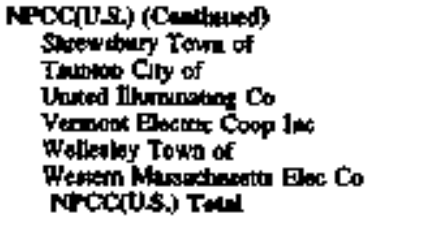 & 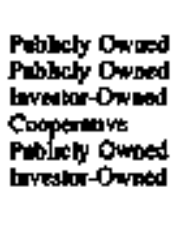 & $\begin{array}{r}2 \\
17 \\
17 \\
0 \\
14 \\
459\end{array}$ & $\begin{array}{r}1 \\
3 \\
26 \\
4 \\
0 \\
36 \\
1,713\end{array}$ & $\begin{array}{r}1 \\
0 \\
0 \\
0 \\
0 \\
16 \\
375\end{array}$ & $\begin{array}{l}7 \\
0 \\
0 \\
0 \\
0\end{array}$ & $\begin{array}{r}3 \\
3 \\
90 \\
1 \\
64 \\
2,555\end{array}$ \\
\hline 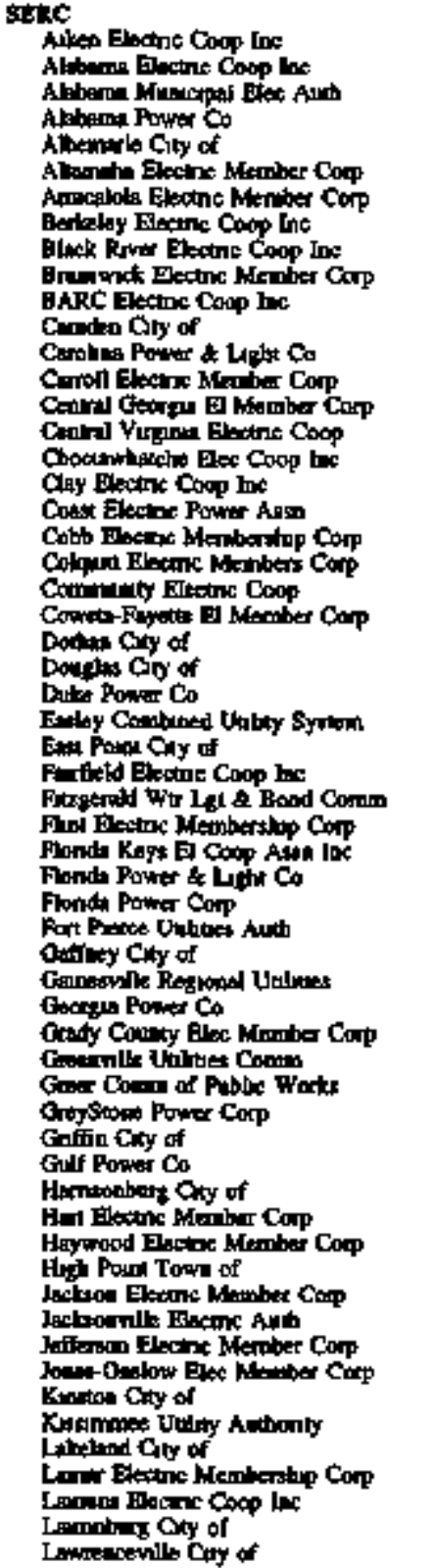 & 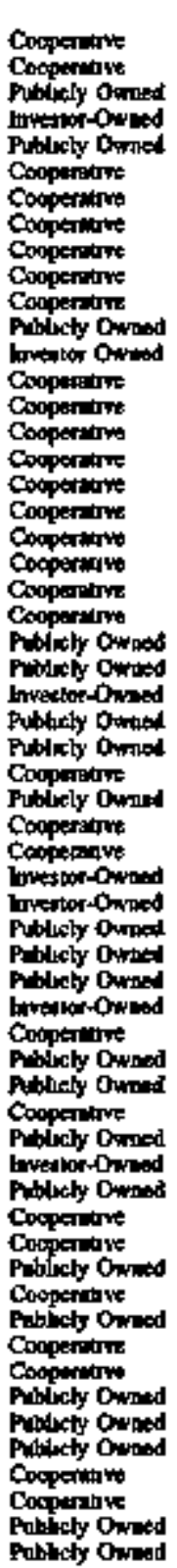 & 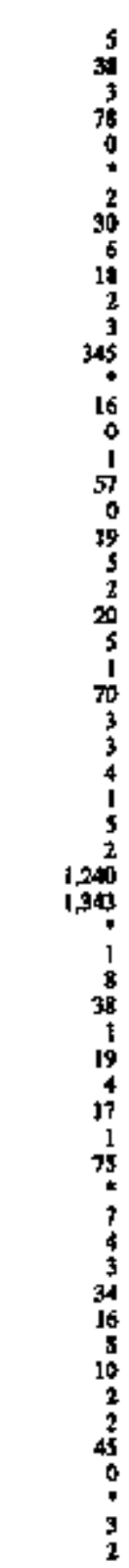 & 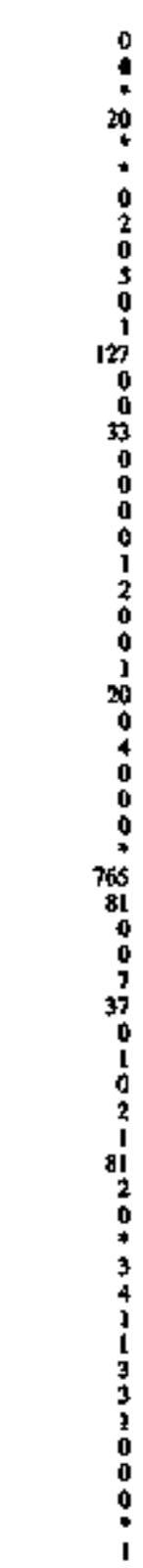 & $\begin{array}{r}0 \\
0 \\
0 \\
0 \\
0 \\
0 \\
0 \\
0 \\
0 \\
0 \\
0 \\
702 \\
0 \\
5 \\
0 \\
0 \\
2 \\
0 \\
0 \\
3 \\
0 \\
0 \\
0 \\
0 \\
0 \\
0 \\
0 \\
0 \\
0 \\
0 \\
0 \\
0 \\
0 \\
0 \\
0 \\
0 \\
0 \\
0 \\
0 \\
0 \\
0 \\
0 \\
0 \\
0 \\
0 \\
0 \\
0 \\
0 \\
0 \\
0 \\
0 \\
0\end{array}$ & $\begin{array}{l}0 \\
0 \\
0 \\
0 \\
0 \\
5 \\
0 \\
0 \\
0 \\
0 \\
0 \\
0 \\
0 \\
0 \\
0 \\
52 \\
0 \\
0 \\
20 \\
0 \\
0 \\
0 \\
0 \\
0 \\
0 \\
0 \\
8 \\
0 \\
0 \\
0 \\
0 \\
0 \\
0 \\
0 \\
0 \\
0 \\
0 \\
0 \\
0 \\
0 \\
0 \\
0 \\
0 \\
0 \\
0 \\
0 \\
0 \\
0 \\
0 \\
0 \\
0 \\
0 \\
0 \\
0 \\
0 \\
0\end{array}$ & 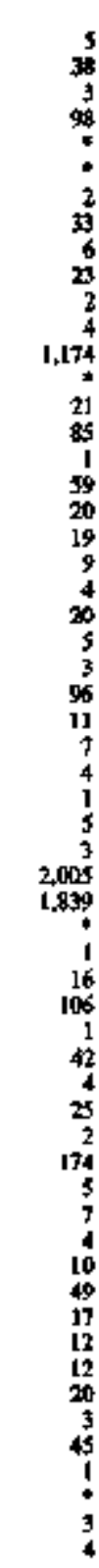 \\
\hline
\end{tabular}

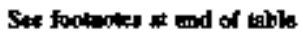


Table 20. U.S. Electric Utility Actuol Peak Logd Reductions by North Anerican Electric Relishility Comcil Region and Hawaii by Closs of Owpership and Sector, 1996 (Megawatts) (Contanued)

\begin{tabular}{|c|c|c|c|c|c|c|}
\hline 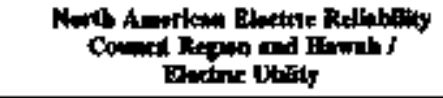 & Plane of & Raldatided & 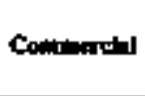 & Indetetel & Ouler & Todal \\
\hline 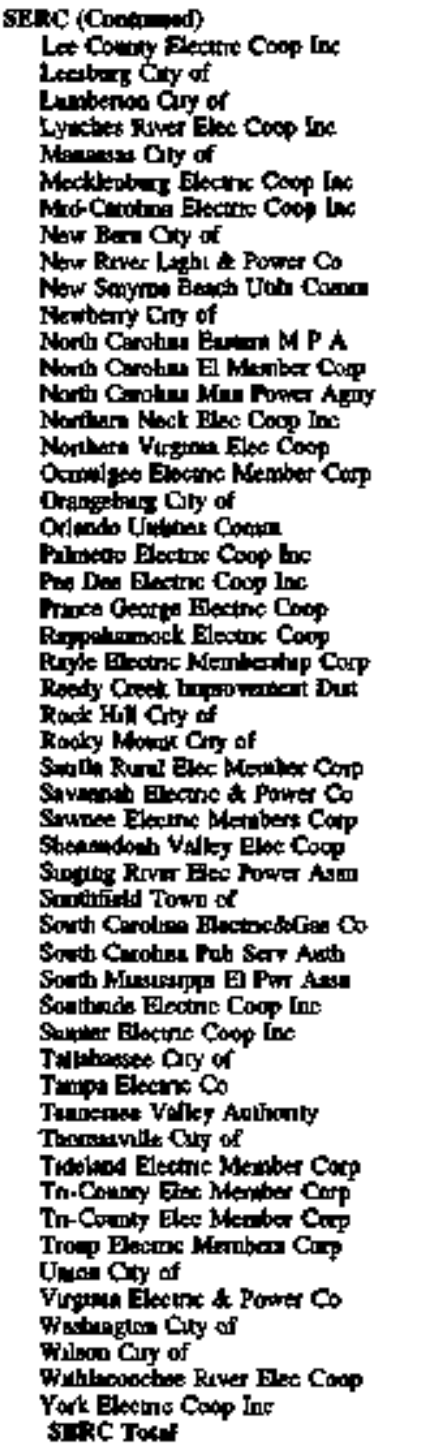 & 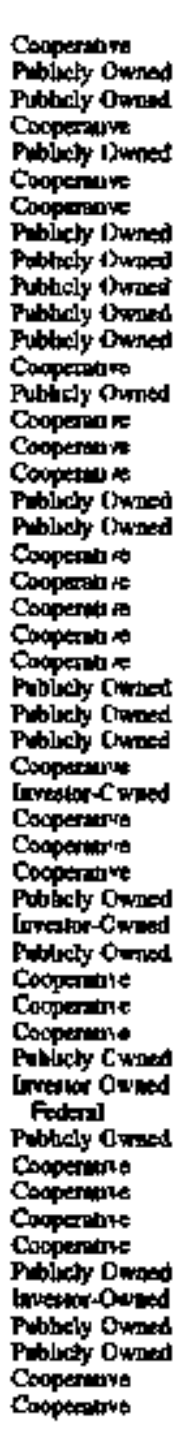 & 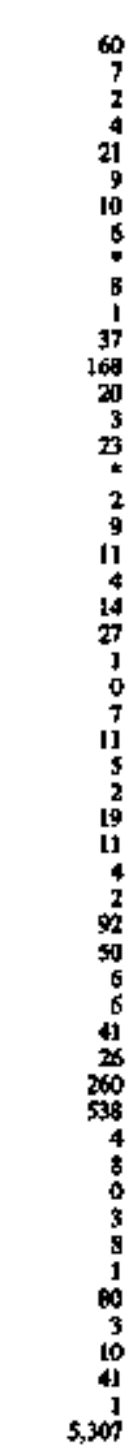 & 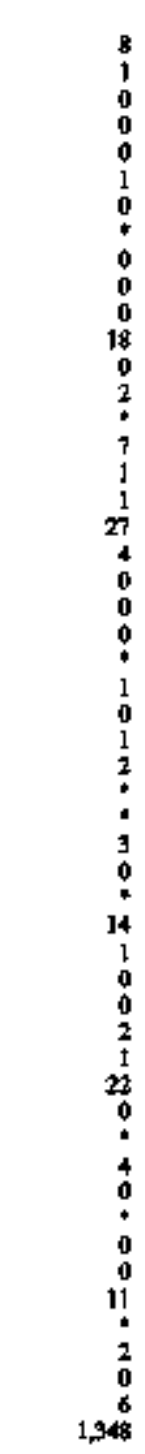 & $\begin{array}{r}0 \\
3 \\
0 \\
0 \\
0 \\
4 \\
0 \\
4 \\
0 \\
0 \\
0 \\
00 \\
80 \\
7 \\
0 \\
3 \\
0 \\
2 \\
0 \\
0 \\
0 \\
0 \\
0 \\
0 \\
0 \\
6 \\
1 \\
0 \\
0 \\
0 \\
0 \\
10 \\
0 \\
0 \\
0 \\
0 \\
0 \\
0 \\
0 \\
0 \\
0 \\
0 \\
0 \\
0 \\
0 \\
0 \\
0 \\
0 \\
0 \\
0 \\
0 \\
0 \\
0 \\
0 \\
0 \\
0 \\
0\end{array}$ & $\begin{array}{r}0 \\
0 \\
0 \\
0 \\
0 \\
0 \\
0 \\
0 \\
0 \\
0 \\
0 \\
0 \\
0 \\
31 \\
0 \\
0 \\
0 \\
2 \\
0 \\
0 \\
0 \\
0 \\
0 \\
0 \\
0 \\
0 \\
0 \\
0 \\
0 \\
0 \\
0 \\
0 \\
0 \\
0 \\
0 \\
0 \\
0 \\
0 \\
0 \\
0 \\
0 \\
0 \\
0 \\
0 \\
0 \\
0 \\
0 \\
0 \\
0 \\
0 \\
0\end{array}$ & 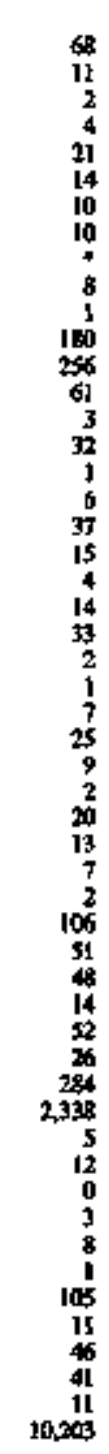 \\
\hline 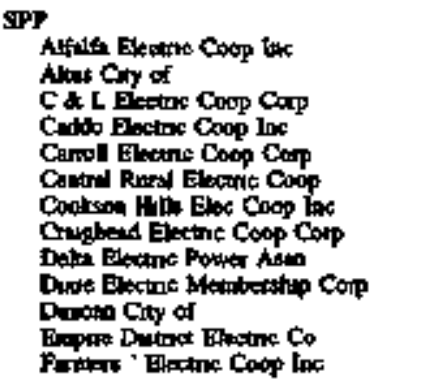 & 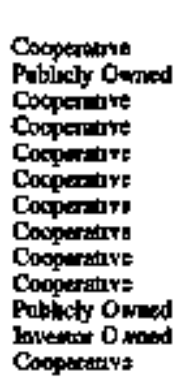 & $\begin{array}{r}0 \\
0 \\
1 \\
9 \\
3 \\
8 \\
0 \\
0 \\
14 \\
0 \\
0 \\
0\end{array}$ & $\begin{array}{l}3 \\
0 \\
0 \\
0 \\
+ \\
+ \\
1 \\
0 \\
0 \\
0 \\
0 \\
0\end{array}$ & $\begin{array}{r}0 \\
* \\
t \\
0 \\
0 \\
2 \\
0 \\
8 \\
2 \\
0 \\
0 \\
21 \\
2\end{array}$ & $\begin{array}{r}0 \\
0 \\
0 \\
10 \\
0 \\
0 \\
0 \\
0 \\
0 \\
0 \\
0 \\
0 \\
0\end{array}$ & $\begin{array}{r}3 \\
+ \\
1 \\
11 \\
9 \\
5 \\
5 \\
5 \\
2 \\
14 \\
5 \\
21 \\
5\end{array}$ \\
\hline
\end{tabular}

Sec footnotes at and of table 
Table 20. U.S. Electric Utility Actual Penk Load Reductions by North American Electric Relipbility Comell Recion and Fowail by Class of Owarership and Sector, 1996 (Megawatts) (Continued)

\begin{tabular}{|c|c|c|c|c|c|c|}
\hline 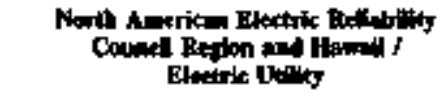 & that of & Resdended & Cumberdid & |n:lestrin| & Onere & TH: \\
\hline 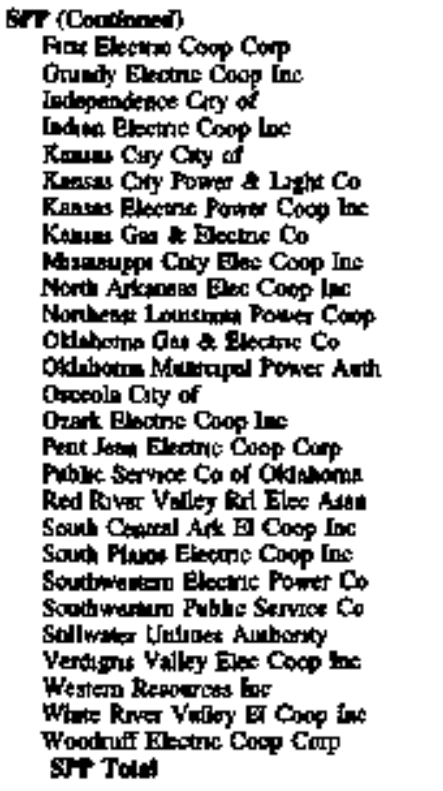 & 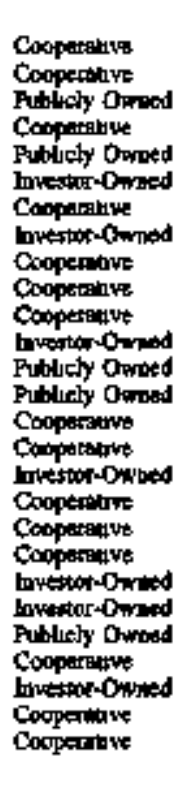 & $\begin{array}{r}14 \\
1 \\
4 \\
2 \\
0 \\
4 \\
4 \\
0 \\
0 \\
5 \\
0 \\
138 \\
1 \\
0 \\
0 \\
3 \\
54 \\
6 \\
0 \\
1 \\
13 \\
27 \\
0 \\
14 \\
12 \\
0 \\
1 \\
381\end{array}$ & $\begin{array}{r}0 \\
0 \\
0 \\
1 \\
0 \\
15 \\
10 \\
0 \\
2 \\
0 \\
3 \\
38 \\
0 \\
0 \\
2 \\
0 \\
3 \\
1 \\
0 \\
0 \\
0 \\
0 \\
0 \\
0 \\
0 \\
16 \\
0 \\
99\end{array}$ & $\begin{array}{r}8 \\
1 \\
0 \\
0 \\
33 \\
15 \\
0 \\
12 \\
0 \\
0 \\
0 \\
5 \\
0 \\
4 \\
0 \\
0 \\
0 \\
1 \\
5 \\
0 \\
0 \\
96 \\
1 \\
1 \\
3 \\
0 \\
4 \\
259\end{array}$ & $\begin{array}{r}0 \\
0 \\
0 \\
0 \\
0 \\
0 \\
18 \\
0 \\
0 \\
0 \\
0 \\
0 \\
0 \\
0 \\
0 \\
0 \\
0 \\
0 \\
0 \\
4 \\
0 \\
45 \\
0 \\
0 \\
83 \\
0 \\
25 \\
185\end{array}$ & $\begin{array}{r}21 \\
2 \\
1 \\
3 \\
33 \\
34 \\
39 \\
13 \\
2 \\
5 \\
231 \\
1 \\
1 \\
2 \\
3 \\
37 \\
2 \\
5 \\
5 \\
13 \\
164 \\
1 \\
15 \\
132 \\
16 \\
30 \\
924\end{array}$ \\
\hline 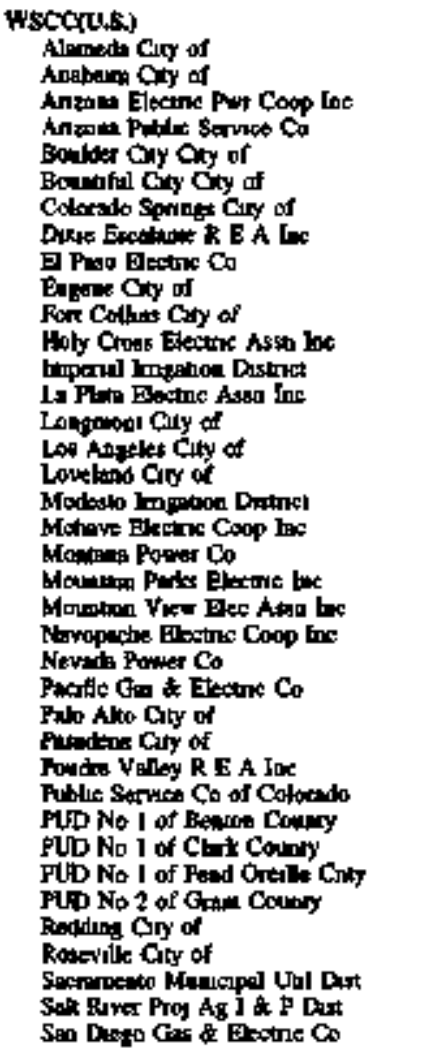 & 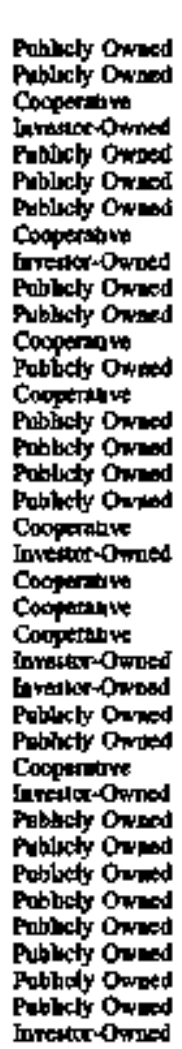 & 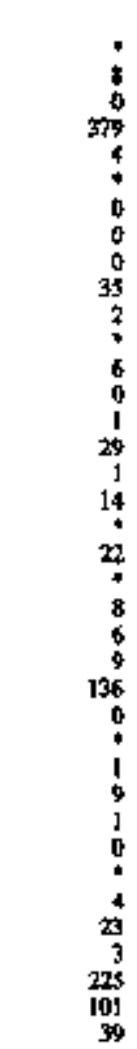 & $\begin{array}{r}1 \\
9 \\
1 \\
127 \\
* \\
* \\
18 \\
7 \\
0 \\
0 \\
1 \\
0 \\
3 \\
49 \\
0 \\
4 \\
27 \\
1 \\
21 \\
1 \\
24 \\
343 \\
6 \\
6 \\
0 \\
27 \\
0 \\
9 \\
104 \\
1 \\
205\end{array}$ & $\begin{array}{r}0 \\
0 \\
0 \\
0 \\
0 \\
7 \\
0 \\
48 \\
3 \\
1 \\
10 \\
0 \\
5 \\
1 \\
8 \\
0 \\
20 \\
0 \\
4 \\
10 \\
0 \\
2 \\
0 \\
580 \\
0 \\
0 \\
01 \\
0 \\
0 \\
1 \\
40 \\
2 \\
0 \\
0 \\
0\end{array}$ & $\begin{array}{c}1 \\
0 \\
0 \\
0 \\
0 \\
0 \\
0 \\
0 \\
0 \\
0 \\
0 \\
0 \\
0 \\
0 \\
0 \\
0 \\
1 \\
0 \\
0 \\
5 \\
0 \\
0 \\
0 \\
0 \\
01 \\
0 \\
0 \\
0 \\
0 \\
0 \\
0 \\
0 \\
0 \\
0 \\
0 \\
0 \\
0\end{array}$ & $\begin{array}{r}2 \\
21 \\
1 \\
506 \\
5 \\
7 \\
4 \\
66 \\
44 \\
2 \\
10 \\
6 \\
5 \\
5 \\
85 \\
2 \\
39 \\
57 \\
11 \\
29 \\
9 \\
33 \\
1.119 \\
6 \\
7 \\
1 \\
298 \\
1 \\
9 \\
1 \\
62 \\
30 \\
5 \\
49 \\
136 \\
295\end{array}$ \\
\hline
\end{tabular}

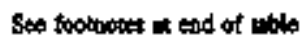


Table 20. U.S. Electric Utility Actual Penk Load Roductions by North Anerican Electric Retiability Courcil Region and Haraid by Class of Ownership and Sector, 1996 (Megawats) (Contunued)

\begin{tabular}{|c|c|c|c|c|c|c|}
\hline 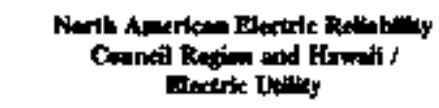 & Clat of & Bothentids & Chorinotal & lodetritel & Other & Toted \\
\hline 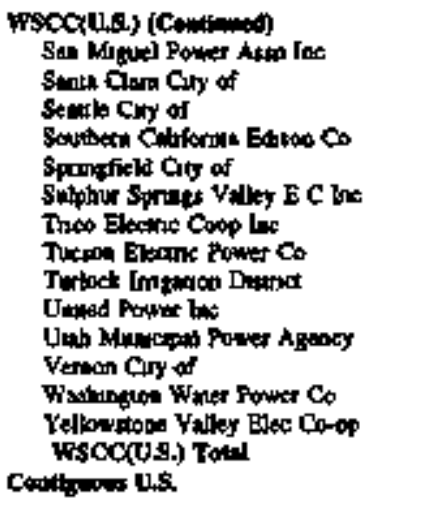 & 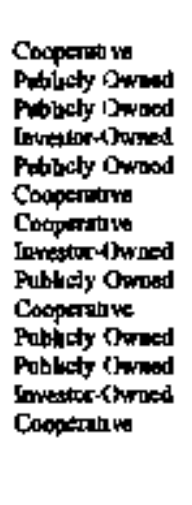 & $\begin{array}{r}0 \\
0 \\
27 \\
364 \\
1 \\
0 \\
0 \\
10 \\
1 \\
2 \\
0 \\
0 \\
73 \\
1 \\
1,540 \\
11,454\end{array}$ & $\begin{array}{r}1 \\
0 \\
30 \\
832 \\
2 \\
0 \\
0 \\
32 \\
1 \\
5 \\
0 \\
0 \\
11 \\
0 \\
2,030 \\
3,069\end{array}$ & $\begin{array}{r}0 \\
7 \\
5 \\
316 \\
\mathbf{t} \\
0 \\
1 \\
6 \\
1 \\
2 \\
0 \\
8 \\
6 \\
0 \\
1,356 \\
9,076\end{array}$ & $\begin{array}{r}0 \\
0 \\
3 \\
82 \\
0 \\
2 \\
0 \\
0 \\
0 \\
0 \\
1 \\
0 \\
0 \\
0 \\
170 \\
681\end{array}$ & $\begin{array}{r}1 \\
70 \\
1.614 \\
4 \\
2 \\
1 \\
38 \\
2 \\
18 \\
1 \\
8 \\
90 \\
1 \\
5.134 \\
29.969\end{array}$ \\
\hline 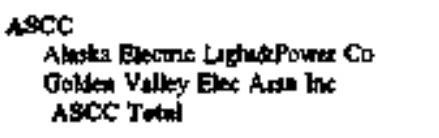 & 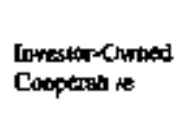 & i & $\begin{array}{l}3 \\
\mathbf{1} \\
3\end{array}$ & 4 & $\begin{array}{l}0 \\
0 \\
0\end{array}$ & $\frac{5}{2}$ \\
\hline 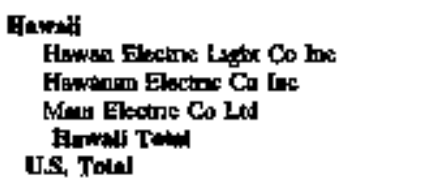 & 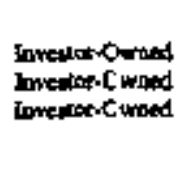 & $\begin{array}{r}2 \\
1 \\
\mathbf{2} \\
3 \\
\mathbf{3 1}, 4 \mathbf{1}\end{array}$ & $\begin{array}{r}1 \\
2 \\
6 \\
5,678\end{array}$ & $\begin{array}{r}0 \\
8 \\
8 \\
8 \\
9,083\end{array}$ & $\begin{array}{r}0 \\
0 \\
0 \\
0 \\
01\end{array}$ & $\begin{array}{r}3 \\
5 \\
9 \\
17 \\
29,893\end{array}$ \\
\hline
\end{tabular}

- Yolue kes the 05

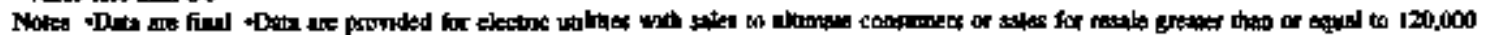

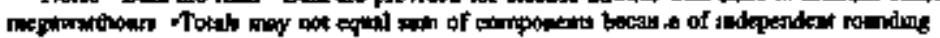

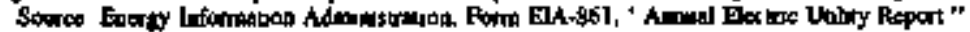




\section{Cost}

Utility costs ${ }^{10}$ for DSM programs are reported by electric utilities using two eategories: ditect utility costs and inditect utility costs. Direct utility costs are those directly attributable to a specific DSM program eategory. Indjrect utility costs are those incurred by utilities that are not directly attributable to a specific DSM program category. Total utility costs are the summation of disect utility costs and indireet utility costs.

In 1996, total utijity costs for large utilities with DSM progranns was $\$ 1.9$ billion, approximately $\$ 519.1$ million less than 1995." For 1997 and 2001, total utlity costs are predicted to stay approximately the same (Table 21).

The declining DSM costs can be attributed partly to competition in the electric power indtustry. In a competitive industry, consumers who use DSM programs will usually incur the costs, tather than electric utiljties financing these programs.

The majority of utilities with DSM program costs spent between 0.1 and 1 percent of electic revenues from sales to yltimate consumers on DSM programs. Among large utilities, 19.9 percent spent less than 0.1 percent of revenues on DSM, 51.4 percent spent between 0.1 and and 1 percent of revenues on DSM, and 2B.7 percent spent more than 1 percent of revenues on DSM. There were 46 cooperatives, 45 investor-owned utilities, and $\mathbf{4 0}$ publicly owned utilities that spent more than 1 percent of revenues on DSM. Of the utilities spending between 0.1 and 1 percent, B8 were publicly owned. 84 were cooperatives, and 63 were investor-owned utilities (Figure 8).

In 1996, the 100 utilities that spent the most on DSM activities accounted for 98.0 percent of total DSM costs; the 50 utilities that spent the most on DSM accounted for 84.8 percent of the total costs; and the top 25 utilities accounted for 69.9 percent (Figure 9).
These 100,50 , and 25 utiljties that had the greatest costs for DSM programs represented $38.4,43.3$, and 25.8 percent, respectively, of total retail sales of electricity in the United States.

In 1996, investor-owned utilities spent the most on DSM, \$1.5 billion, followed by 12 gublicly owned utilities, \$159.8 million; Federally owned utilities, \$101.6 milion and cooperatives, \$92.3 million. Publicly owned utilities predicted a 13.8 percent increase for 1997. For 2001, all classes of ownership anticipated spending reductions excepl cooperatives (Table 21).

Direct Utility Costs are those identified specifically with one of the DSM program categories (i.e., energy efficiency, direct load control, interruptible load control, other load management, other DSM programs, or load building). In 1996, dírect utility costs for large utilities was $\$ 1.6$ billion. Of direct utility costs, 64.8 percent were for enersy effieiency programs, anounting to $\$ 1.1$ billion (Table 23). Direct utility costs reported by utilities do not inclode lost revenue as a result of offering customers incerruptible rates.

Among the NERC regions, SERC had the greatest shate of direct utility costs, $\$ 500.1$ mitlion, mainly because within the SERC there were a number of arge utilities promoting DSM programs.

Indirect Utility Costs are vtility costs that may not be meaningfully identified with any particular DSM program category. Indirect costs coutd be attributable to one of several accounting cost categories (i.e., administrgtive, marketing, monitoring zod evaluation, utility-earned incentjves, ${ }^{13}$ or other ${ }^{14}$ ). Inditect utility costs for 1996 were $\$ 278.6$ million, with the greatest portion of these costs for administrative costs.

Among the NERC regions, WSCC had the highest share of indirect utility costs, $\$ 91.9$ million, followed by SERC with $\$ 50,9$ miltion (Table 24 ).

10 Uabitieg aye required to report nonutility costs (nonutility costs are thoge incurred by the consumar, sach as installation of an energy efficient appliancey or by the setailer or mapafucturer of energy efficient prodnets), but they are not included in this report because in many cases utilities cannot accurately estinate these costs.

If \$mall utilitios are not included in tajs sectios af they report only total ouility cosl and not a breakdown into direct and indirect costs.

12 The large amoonl of spending repotted by Fedetaly owbed ulilities nay be misleading. Both the Tennegsee Valley Authority and Boneville Power Administration encourage ntilities to use bSM, and finance their proframs.

13 Unilly-earat dncertives are not included is this publicatios.

14 Other costs include the indirocl cogt of DSM thal capnol be zetribnted to any pather cost category, particulyrly research and develop. ment. 
Figure 8. U.S. Electric Utility DSU Program Conts as a Percentage of Retall Revenue by Number of Utilities with DSW Coste, 1996

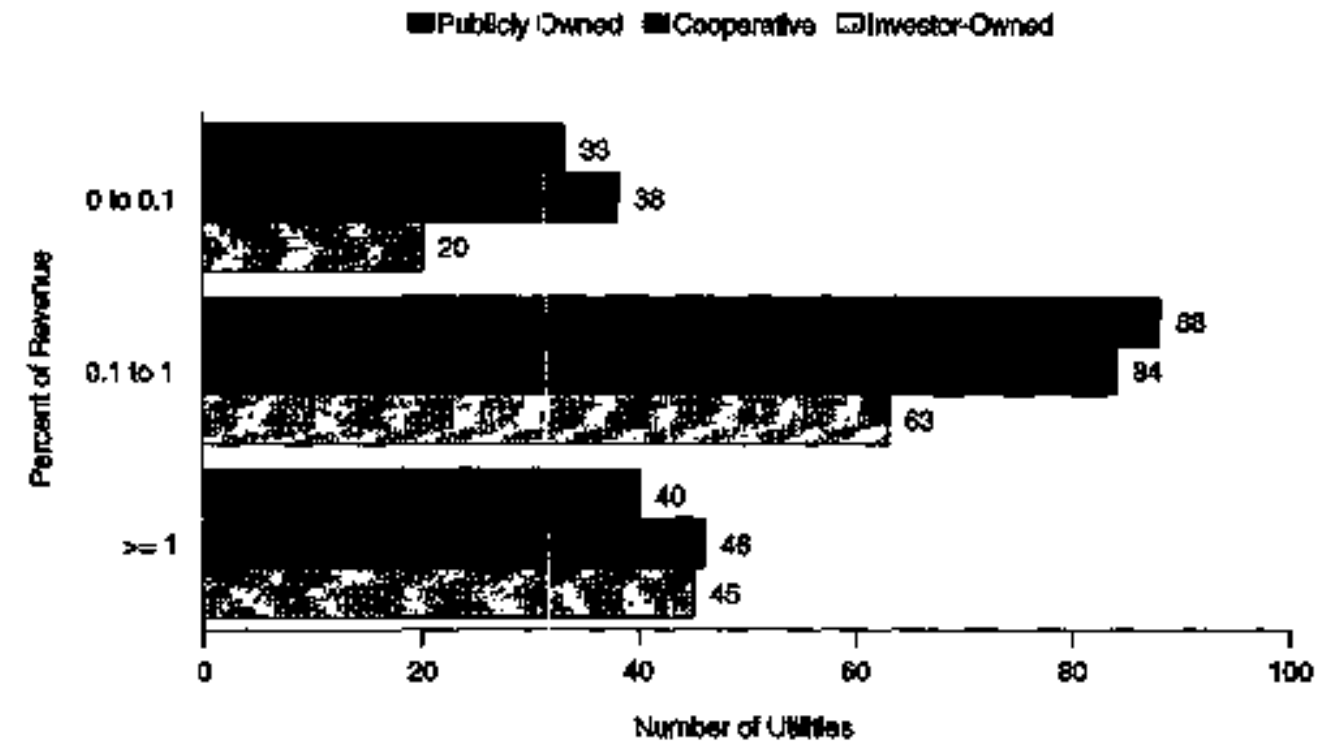

Source: Energy Information Administration, Form EIA-861, "Annuat Electric Utility Repont."

Figure 9. The Top 25, 50, and 100 U.5. Electirc Utilites with the Greateat DSW Program Cost: by Class of Ownership, 1996

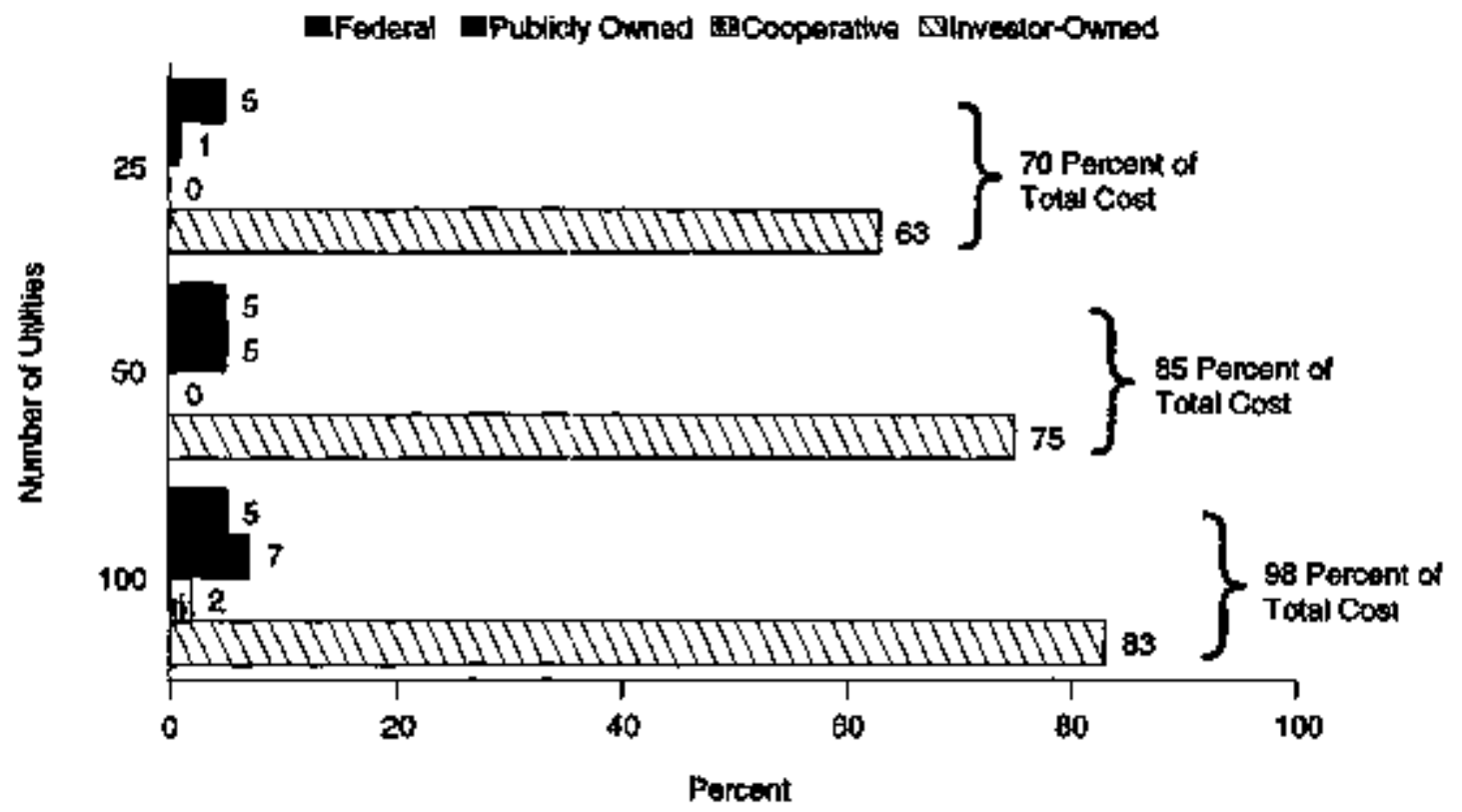

Note: Totals may not equal sum of somponents because of independent rounding No cooperatives were included in the top 25 or 50 utilitios.

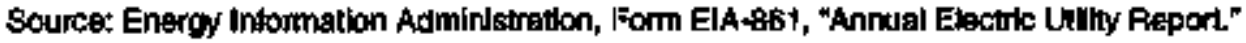


Table 21. U.S. Ekectic Utility DSM Program Cost by Clase of Onrnership, 1992 Throngh 1996, 1997 , and 2901

(Thonsand Dollars)

\begin{tabular}{|c|c|c|c|c|c|c|c|}
\hline \multirow{2}{*}{ Clune of Owatralp } & \multicolumn{5}{|c|}{ Elrorkel Costr } & \multicolumn{2}{|c|}{ Projocted Conts } \\
\hline & $\lg 92$ & 1593 & 1994 & 1905 & $13 \%$ & L97 & 2:01 \\
\hline $\begin{array}{l}\text { Inwoter-Dwned } \\
\text { Pubhely Owmed } \\
\text { Coopersine } \\
\text { Pederal } \\
\text { US. Tolal }\end{array}$ & $\begin{array}{r}1,918,803 \\
163,075 \\
81,553 \\
184,663 \\
2,34,064\end{array}$ & $\begin{array}{r}2,251,277 \\
166,774 \\
87,618 \\
27,714 \\
2,743,513\end{array}$ & $\begin{array}{r}2,290,646 \\
183,274 \\
05,244 \\
246,493 \\
2,716,457\end{array}$ & $\begin{array}{r}1,951,824 \\
18,294 \\
99,013 \\
191,020 \\
2,01,71\end{array}$ & $\begin{array}{r}1,548,510 \\
1,59,849 \\
92,258 \\
101,580 \\
1,94,157\end{array}$ & $\begin{array}{r}1,615,891 \\
181,890 \\
97,200 \\
81,329 \\
1,976,390\end{array}$ & $\begin{array}{r}1,549,590 \\
199,962 \\
97,521 \\
7.773 \\
1014,947\end{array}$ \\
\hline
\end{tabular}

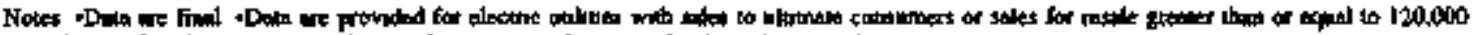

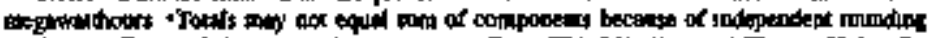

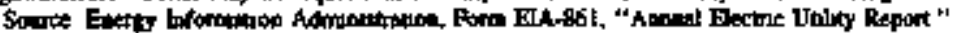




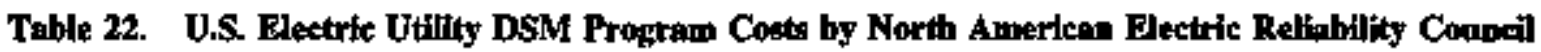
Region and Hawnï by Class of Ownershtp, 1995, 1996, 1997, and 2001 (Thousand Doilars)

\begin{tabular}{|c|c|c|c|c|c|}
\hline \multirow{2}{*}{ 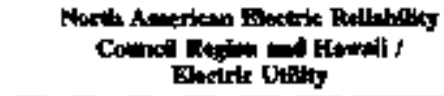 } & \multirow{2}{*}{$\begin{array}{c}\text { Qust of } \\
\text { Omertip }\end{array}$} & \multicolumn{2}{|c|}{ Finodead Cets } & \multicolumn{2}{|c|}{ Preltected Costi } \\
\hline & & 10s: & Iens & 359 & 2000 \\
\hline 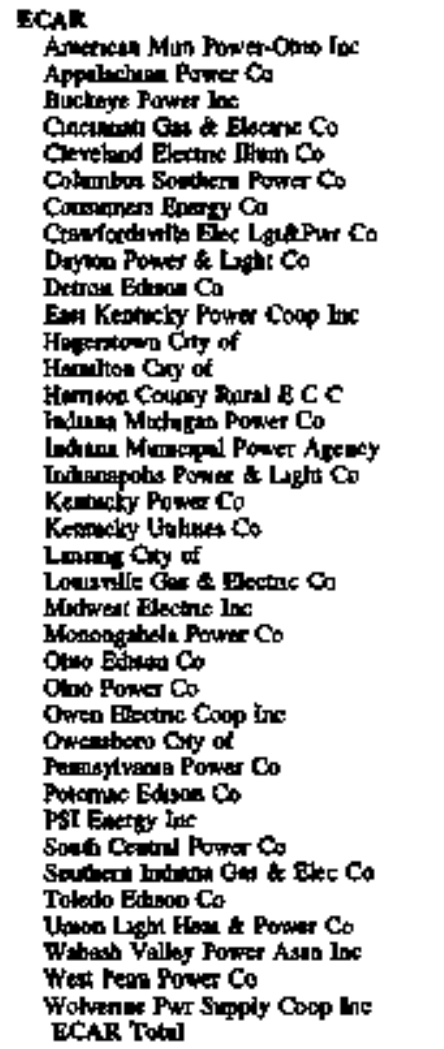 & 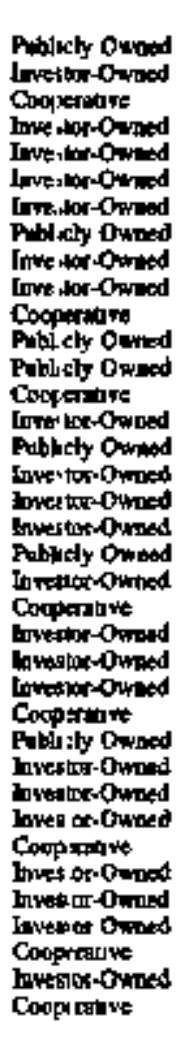 & 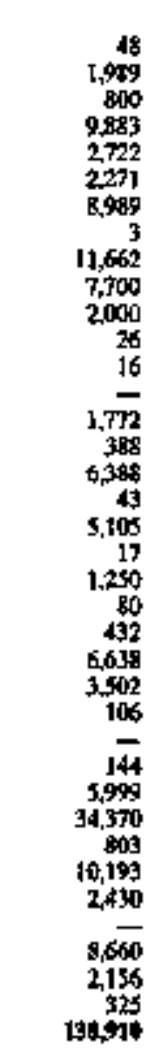 & 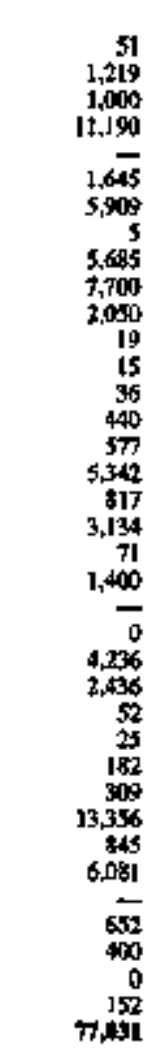 & 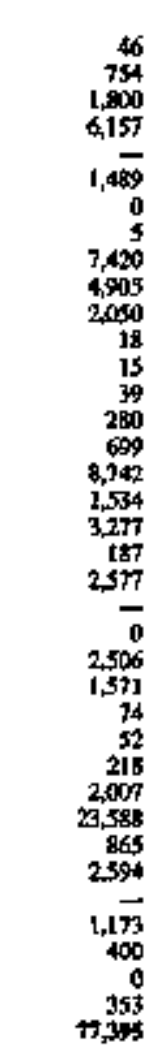 & $\begin{array}{r}50 \\
858 \\
3,500 \\
7,233 \\
2,144 \\
0 \\
3 \\
7,420 \\
3,810 \\
0 \\
0 \\
25 \\
39 \\
369 \\
120 \\
0 \\
1,250 \\
3,528 \\
190 \\
5,900 \\
0 \\
1,618 \\
2,643 \\
91 \\
30 \\
327 \\
2,047 \\
25,447 \\
980 \\
1,941 \\
1,378 \\
400 \\
0 \\
135 \\
7,494\end{array}$ \\
\hline 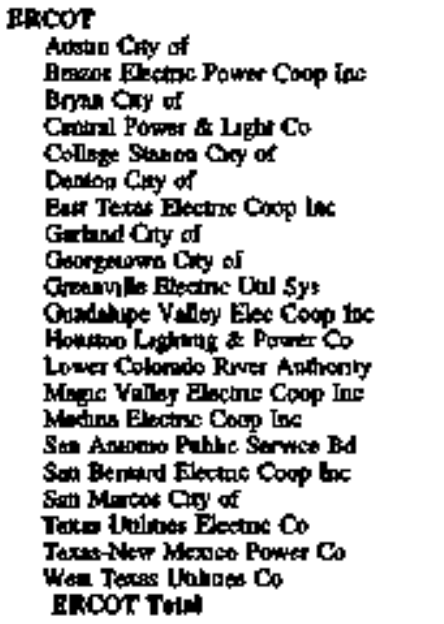 & 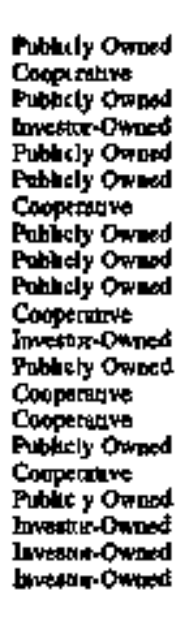 & $\begin{array}{r}13,282 \\
1,415 \\
498 \\
7,549 \\
95 \\
71 \\
\frac{-}{614} \\
38 \\
56 \\
243 \\
21,215 \\
6,060 \\
488 \\
57 \\
472 \\
415 \\
27 \\
14,307 \\
1,194 \\
2,680 \\
70,21\end{array}$ & 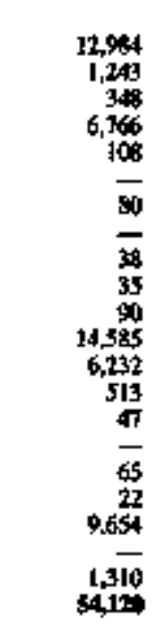 & 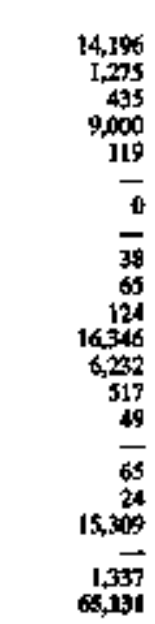 & 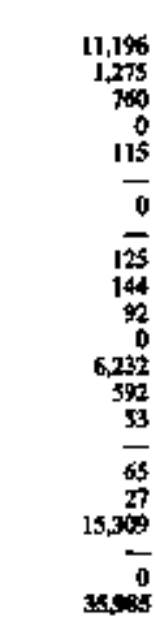 \\
\hline 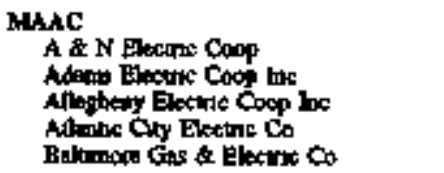 & 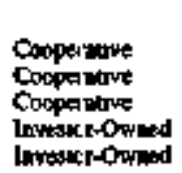 & $\begin{array}{r}349 \\
605 \\
706 \\
3,536 \\
53,179\end{array}$ & $\frac{143}{3,789}$ & $\frac{145}{3,831}$ & $\frac{152}{4,125}$ \\
\hline
\end{tabular}

Soc fosthites an end of pole 
Table 22. U.S. Blectric Utility RSM Program Costs by North American Electric Reliability Conaneil Region and Howall by Class of Ownership, 1995, 19\%, 1997, and 2001 (Thousend Dollars) (Conlinued)

\begin{tabular}{|c|c|c|c|c|c|}
\hline \multirow{2}{*}{ 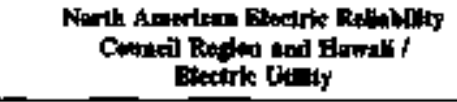 } & \multirow{2}{*}{ otess of } & \multicolumn{2}{|c|}{ Elstodelal Conde } & \multicolumn{2}{|c|}{ Projested Couls } \\
\hline & & $19 \% 5$ & tw\% & $19 \$$ & 2001 \\
\hline 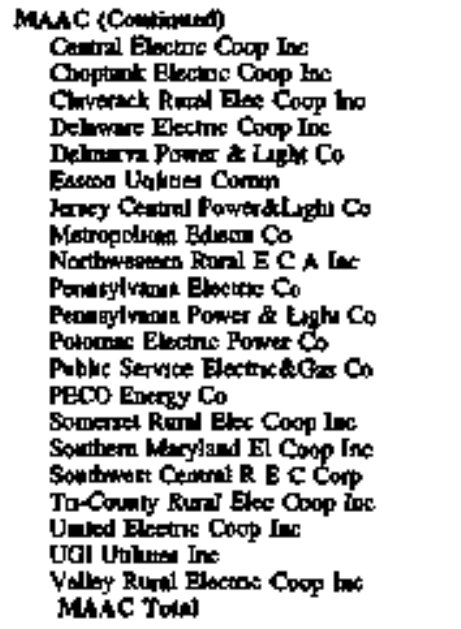 & 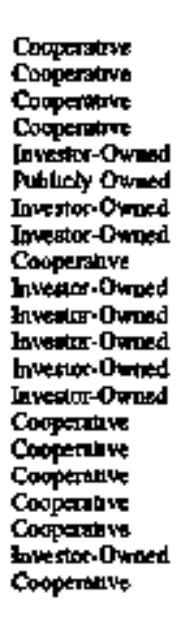 & $\begin{array}{r}219 \\
265 \\
69 \\
772 \\
8,966 \\
70 \\
30,895 \\
4,320 \\
356 \\
4,790 \\
11,434 \\
118,955 \\
46,486 \\
8,771 \\
142 \\
5.785 \\
66 \\
61 \\
144 \\
110 \\
116 \\
300,347\end{array}$ & 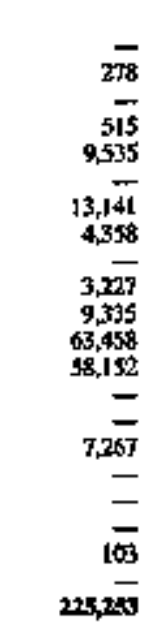 & 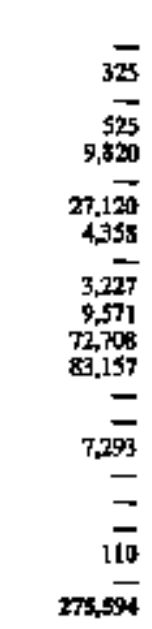 & $\begin{array}{r}456 \\
568 \\
9,870 \\
19,500 \\
4,359 \\
\overline{0} \\
9,511 \\
70,290 \\
124,351 \\
= \\
8,49 \\
= \\
= \\
110 \\
29,790\end{array}$ \\
\hline 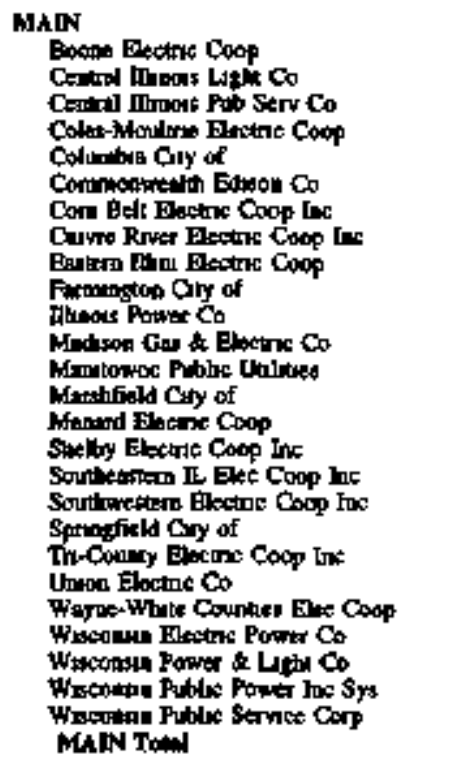 & 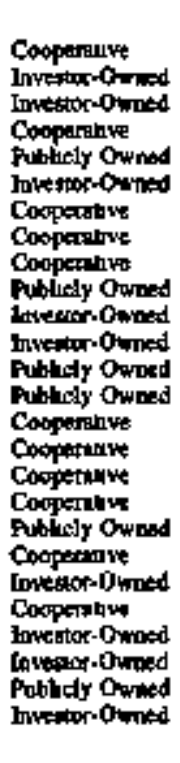 & $\begin{array}{r}94 \\
2065 \\
566 \\
150 \\
655 \\
4960 \\
210 \\
39 \\
92 \\
101 \\
19 \\
4,764 \\
230 \\
130 \\
130 \\
35 \\
2 \\
150 \\
525 \\
115 \\
11,718 \\
26 \\
21,913 \\
13,999 \\
811 \\
14,760 \\
74,094\end{array}$ & $\begin{array}{r}56 \\
2967 \\
130 \\
130 \\
834 \\
8,500 \\
177 \\
45 \\
92 \\
-7 \\
4,356 \\
97 \\
134 \\
122 \\
27 \\
4 \\
156 \\
467 \\
14 \\
12,762 \\
33 \\
19,160 \\
8,347 \\
493 \\
11,360 \\
74,356\end{array}$ & 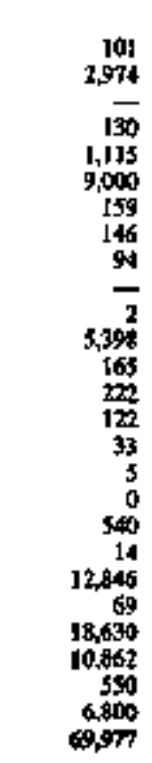 & 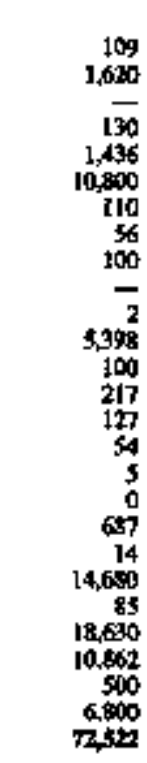 \\
\hline 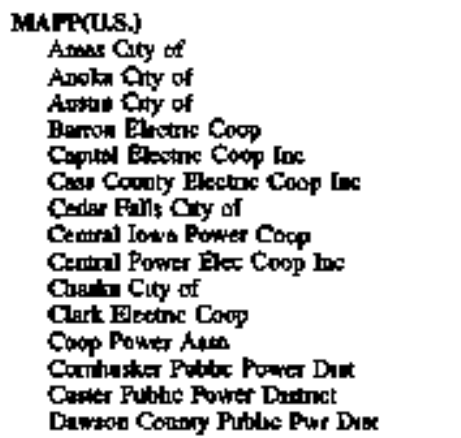 & 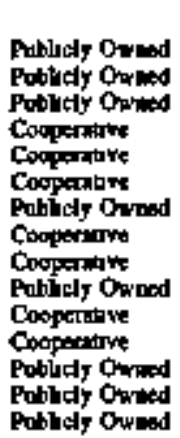 & $\begin{array}{r}250 \\
71 \\
288 \\
46 \\
44 \\
130 \\
300 \\
1,431 \\
90 \\
77 \\
27 \\
8,468 \\
57 \\
13 \\
30\end{array}$ & $\begin{array}{r}251 \\
143 \\
170 \\
396 \\
46 \\
136 \\
300 \\
1,574 \\
100 \\
105 \\
115 \\
9,096 \\
= \\
\bar{n}\end{array}$ & $\begin{array}{r}252 \\
143 \\
160 \\
332 \\
48 \\
198 \\
300 \\
1,725 \\
100 \\
110 \\
116 \\
31,322 \\
\frac{110}{23}\end{array}$ & $\begin{array}{r}77 \\
154 \\
168 \\
272 \\
56 \\
157 \\
300 \\
1,884 \\
100 \\
134 \\
116 \\
13,025 \\
= \\
75\end{array}$ \\
\hline
\end{tabular}

Seo thotomat at end of able 
Table 22. U.S. Electric Utilty DSM Propram Couls by North Ameritan Electric Reltability Conncil Retion and Hawriti by Cluss of Ownership, 1995, 1996, 1997, and 2001 (Thousand Doflars) (Contunued)

\begin{tabular}{|c|c|c|c|c|c|}
\hline \multirow{2}{*}{ 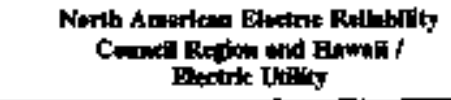 } & \multirow{2}{*}{ 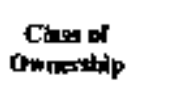 } & \multicolumn{2}{|c|}{ Fithried Cats } & \multicolumn{2}{|c|}{ Prinjetind Coot } \\
\hline & & 1925 & 1906 & Is; & 2001 \\
\hline 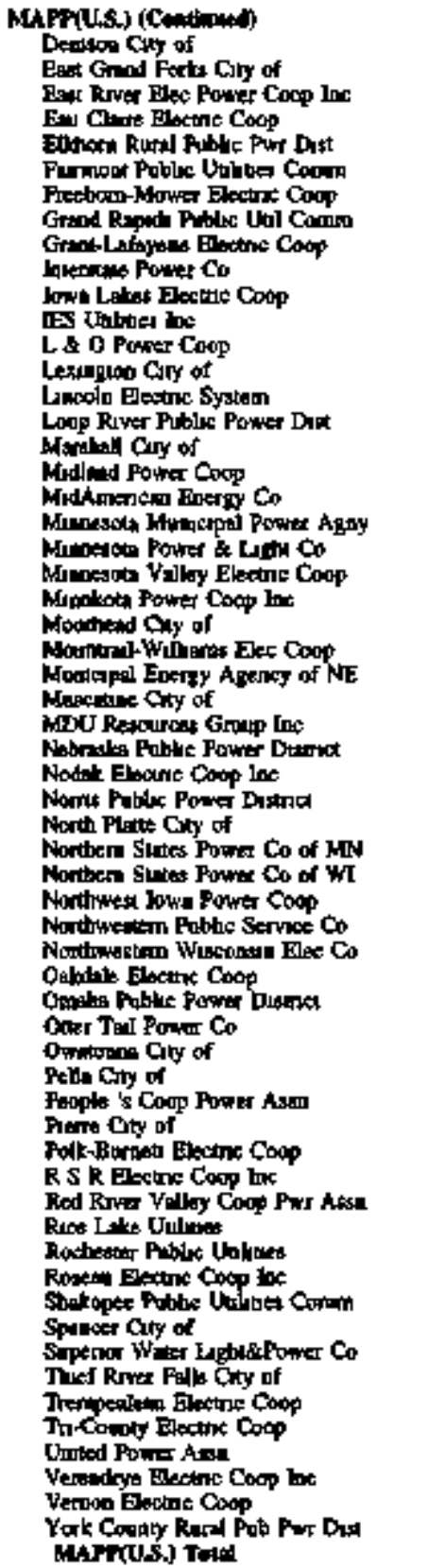 & 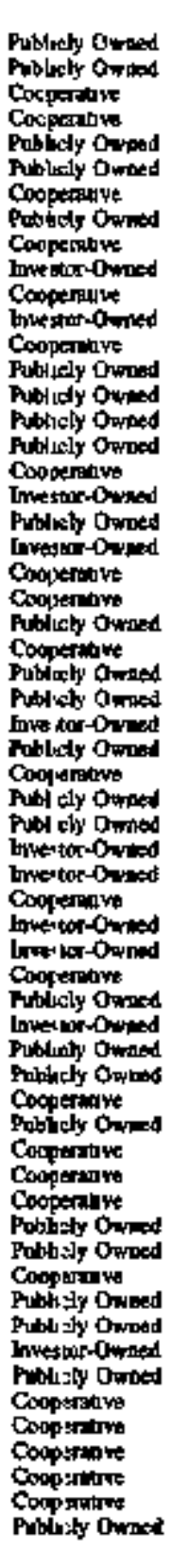 & 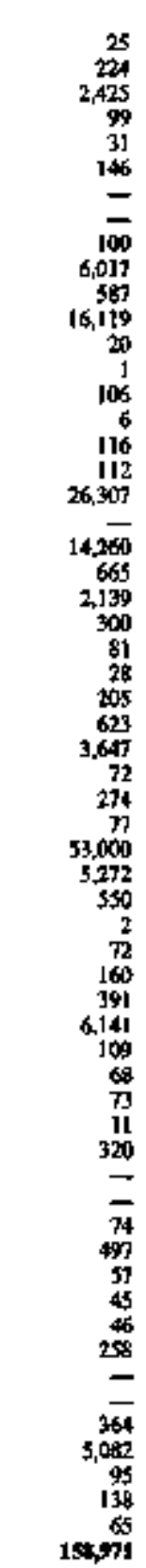 & 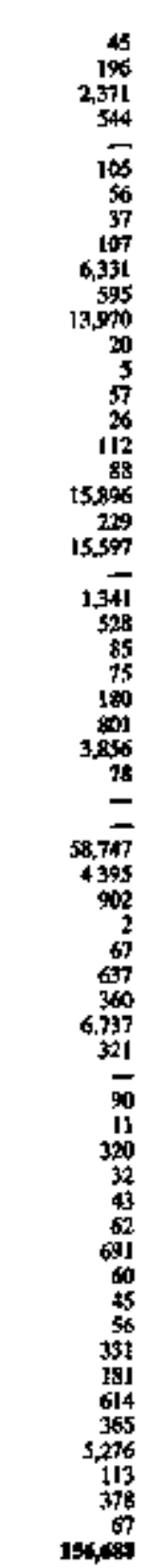 & 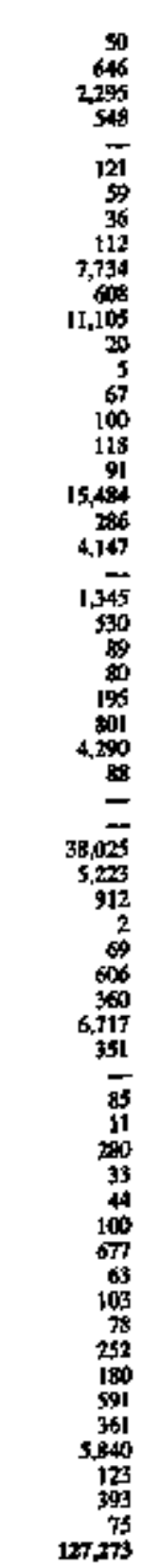 & 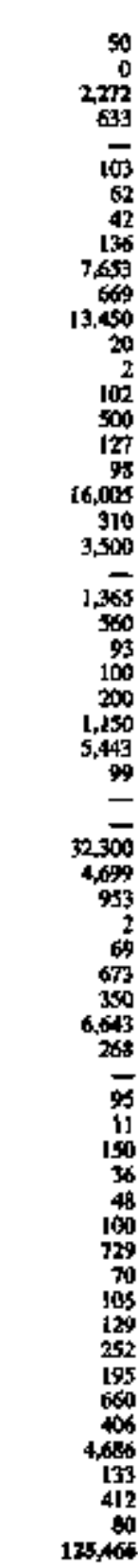 \\
\hline 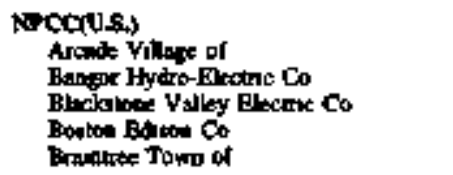 & 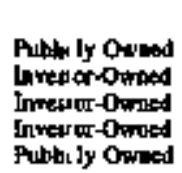 & $\begin{array}{r}25 \\
609 \\
0 \\
32,595 \\
198\end{array}$ & $\begin{array}{r}5 \\
164 \\
1.580 \\
15.916 \\
203\end{array}$ & $\begin{array}{r}5 \\
528 \\
2,45 \\
21,318 \\
211\end{array}$ & $\begin{array}{r}5 \\
828 \\
2,455 \\
21,318 \\
221\end{array}$ \\
\hline
\end{tabular}

Sec footnotes at end of table 
Table 22. US. Electric Utiky DSM Progran Casts by North American Electric Reliability Conncid Regiton and Fawail by Cless of Ownership, 1995, 1996, 1997, and 2001

(Thousand Dollars) (Continued)

\begin{tabular}{|c|c|c|c|c|c|}
\hline \multirow{2}{*}{ 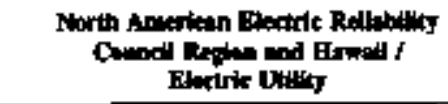 } & \multirow{2}{*}{ 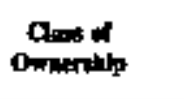 } & \multicolumn{2}{|c|}{ Elaterdal Coots } & \multicolumn{2}{|c|}{ Fragesed Coits } \\
\hline & & $19 \%$ & 159 & 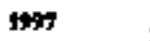 & 201 \\
\hline 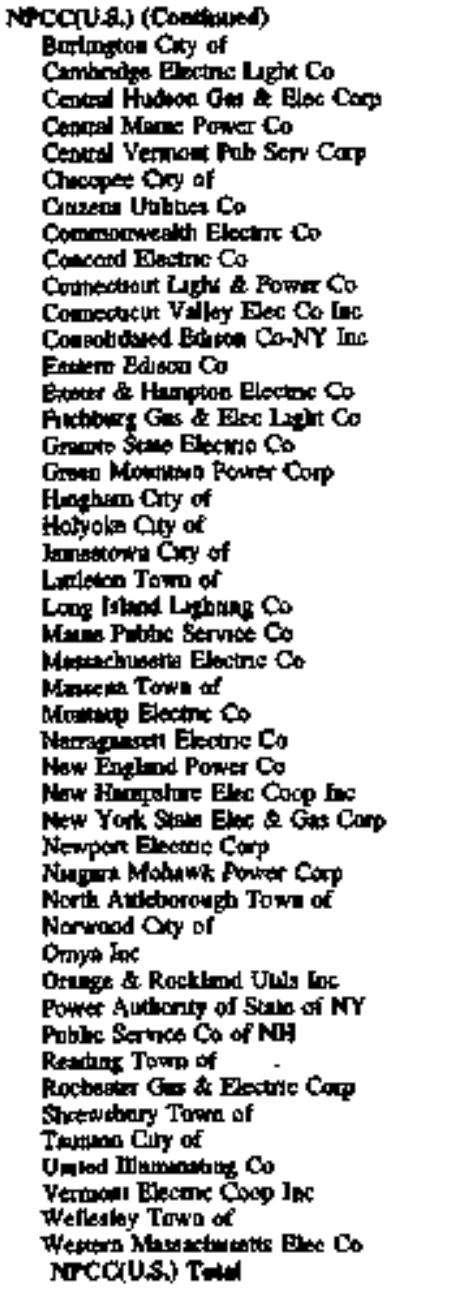 & 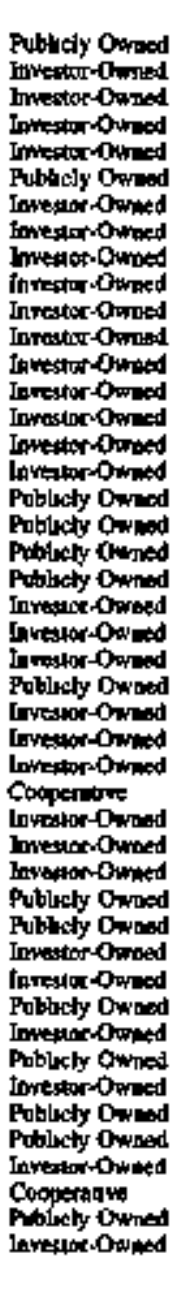 & 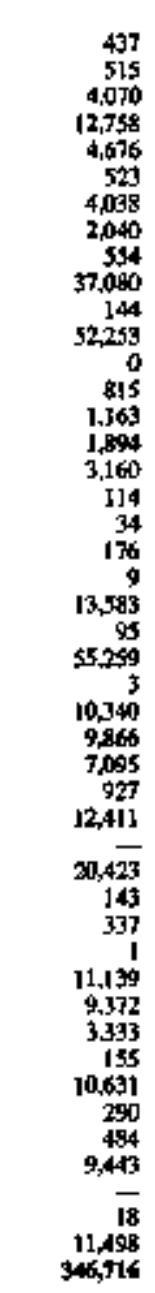 & 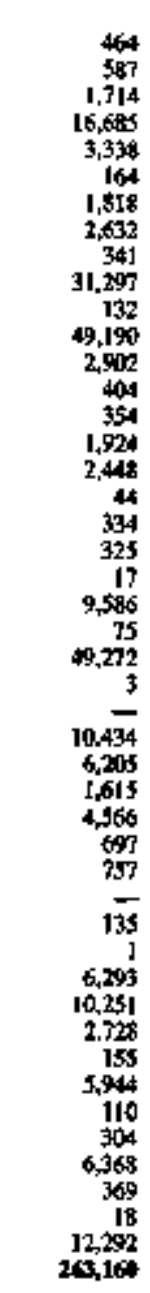 & 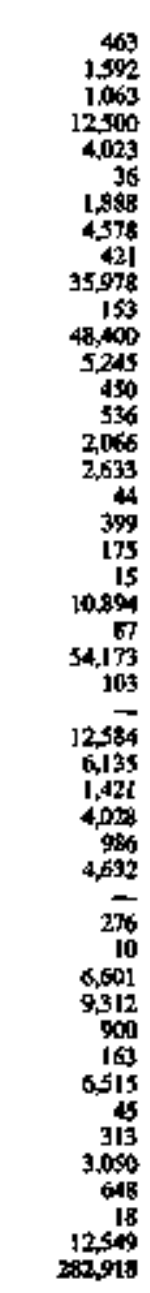 & 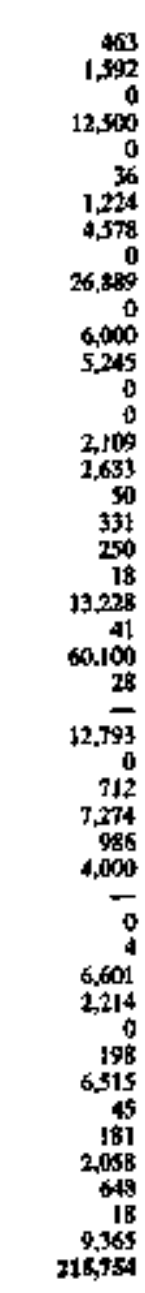 \\
\hline 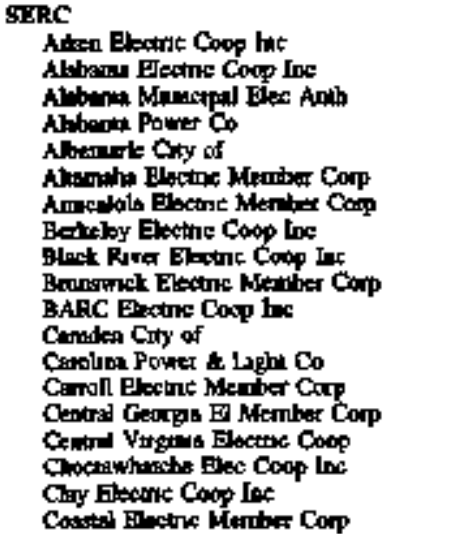 & 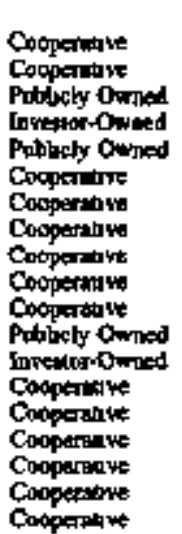 & $\begin{array}{r}263 \\
1042 \\
110 \\
45,166 \\
40 \\
13 \\
78 \\
762 \\
310 \\
697 \\
98 \\
56 \\
56,600 \\
73 \\
118 \\
61 \\
190 \\
2,930 \\
163\end{array}$ & $\begin{array}{r}818 \\
1,269 \\
110 \\
51,546 \\
36 \\
0 \\
30 \\
796 \\
215 \\
671 \\
98 \\
59 \\
51,500 \\
15 \\
108 \\
76 \\
189 \\
7,947 \\
-\end{array}$ & $\begin{array}{r}625 \\
1,323 \\
145 \\
57,711 \\
46 \\
0 \\
32 \\
902 \\
222 \\
566 \\
98 \\
21 \\
50600 \\
3 \\
130 \\
175 \\
180 \\
3,417 \\
-\end{array}$ & $\begin{array}{r}990 \\
0 \\
195 \\
70,666 \\
70 \\
0 \\
36 \\
600 \\
295 \\
322 \\
89 \\
72 \\
50,800 \\
3 \\
186 \\
156 \\
190 \\
4,179 \\
-\end{array}$ \\
\hline
\end{tabular}

See footrote a end of toble 
Table 22. U.S. Electric Utility DSM Program Costs by North American Electric Religlility Coancil Region and Hawall by Cless of Ownership, 1995, 19\%6, 1997, and 2001 (Thousand Dollars) (Contınued)

\begin{tabular}{|c|c|c|c|c|c|}
\hline \multirow{2}{*}{ 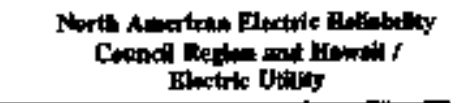 } & \multirow{2}{*}{ 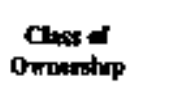 } & \multicolumn{2}{|c|}{ Hetodtal Costs } & \multicolumn{2}{|c|}{ Reofuctad Ceth } \\
\hline & & 15\% & $19 \%$ & $16 \mathrm{H}$ & 2,01 \\
\hline 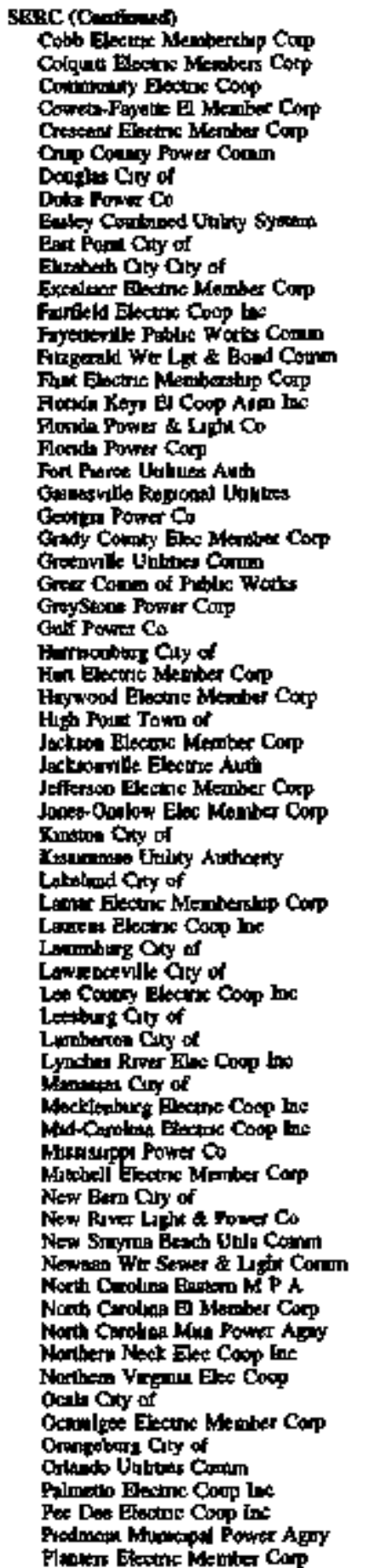 & 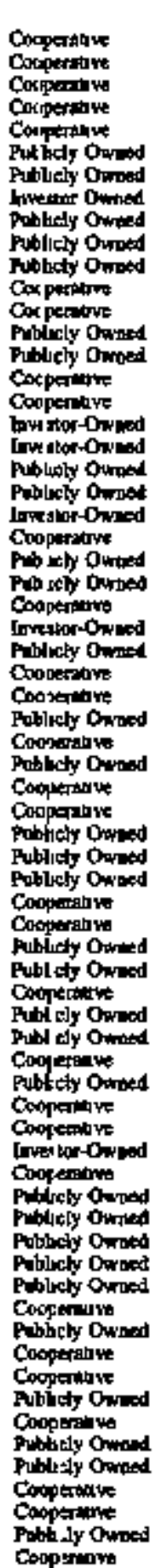 & 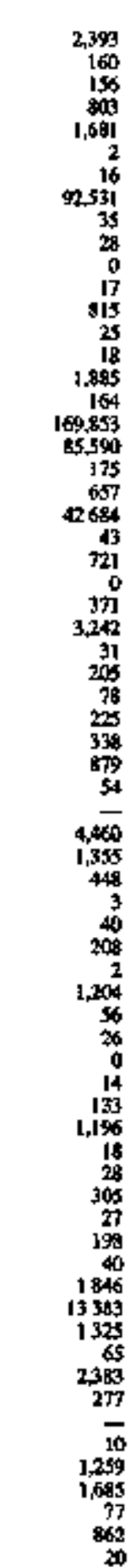 & 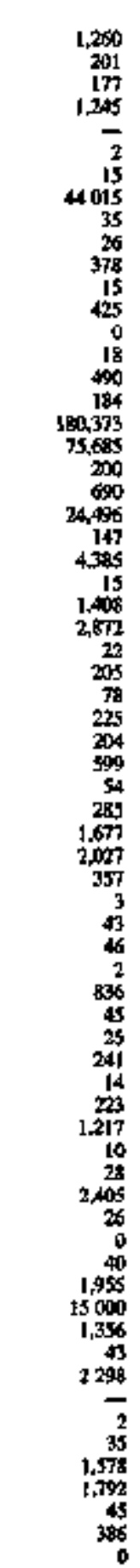 & 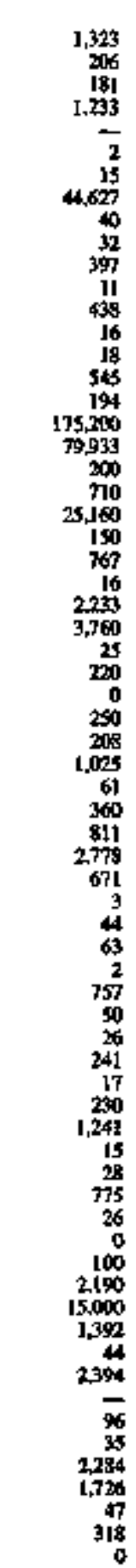 & 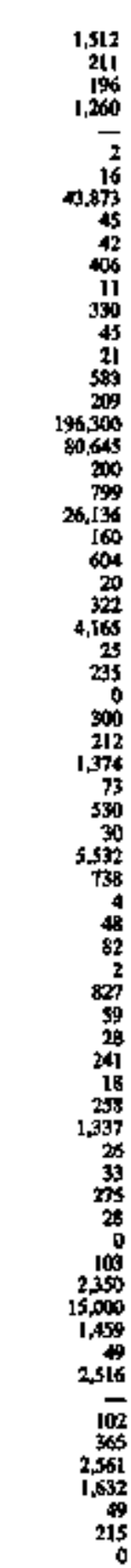 \\
\hline
\end{tabular}

Sos tootnetes at end of nolle 
Table 22. U.S. Electric Utility DSM Program Costs by North American Electric Reliability Council Re-ion and Hawaii by Class of Ownership, 1995, 19\%6, 1997, and 2001 (Thousand Dollars) (Continued)

\begin{tabular}{|c|c|c|c|c|c|}
\hline \multirow{2}{*}{ 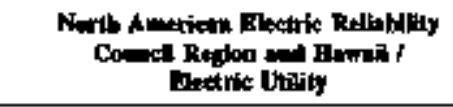 } & \multirow{2}{*}{ Cres of } & \multicolumn{2}{|c|}{ Altserkal Coot } & \multicolumn{2}{|c|}{ Proficind Cecta } \\
\hline & & Ind & 195 & $19 \%$ & 2.11 \\
\hline 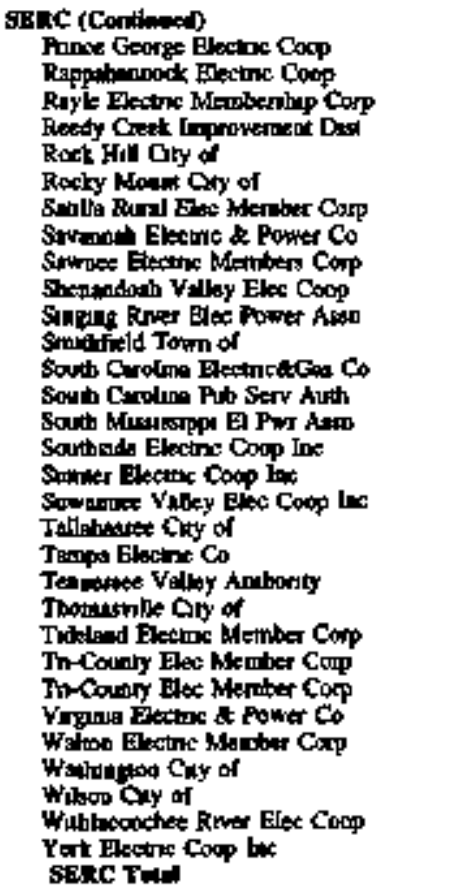 & 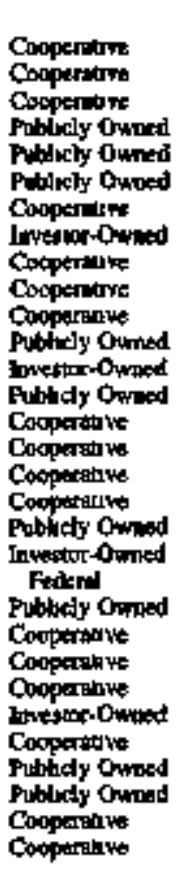 & 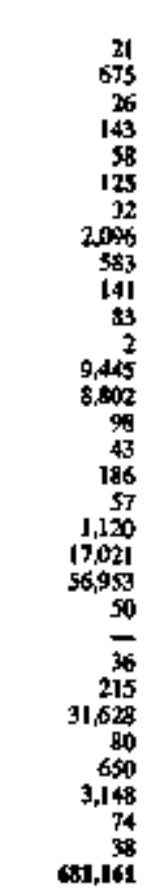 & $\begin{array}{r}26 \\
651 \\
27 \\
143 \\
28 \\
125 \\
32 \\
0 \\
622 \\
112 \\
69 \\
92 \\
1,836 \\
9,106 \\
130 \\
44 \\
167 \\
21 \\
860 \\
18,697 \\
5,945 \\
2 \\
150 \\
32 \\
222 \\
24,219 \\
70 \\
62 \\
2,660 \\
72 \\
35 \\
651,837\end{array}$ & 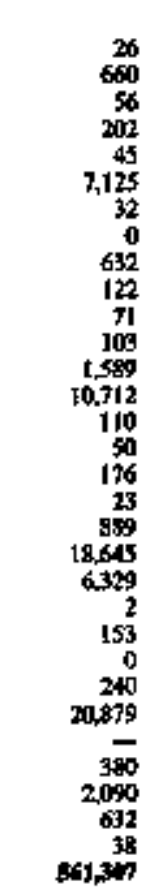 & 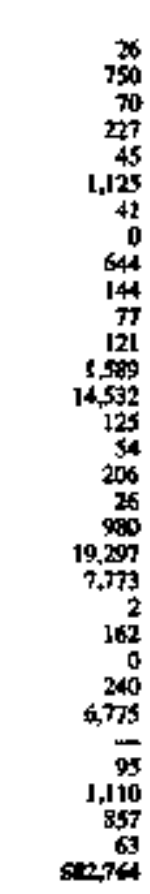 \\
\hline 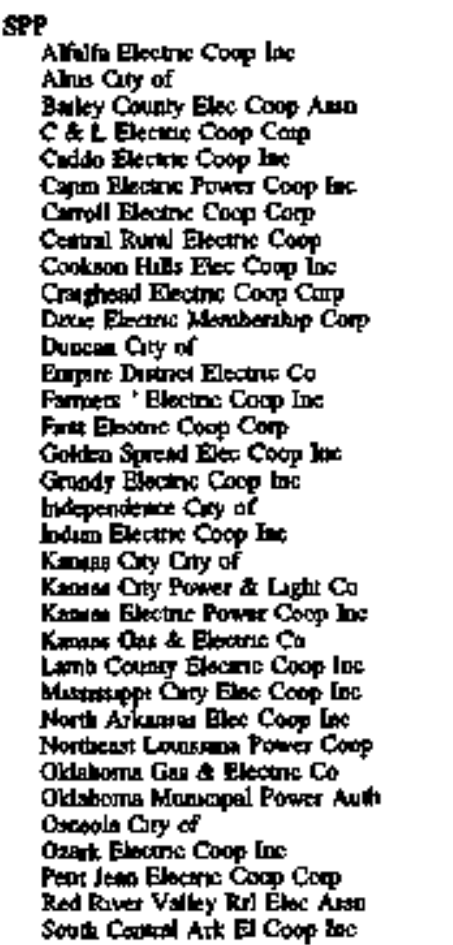 & 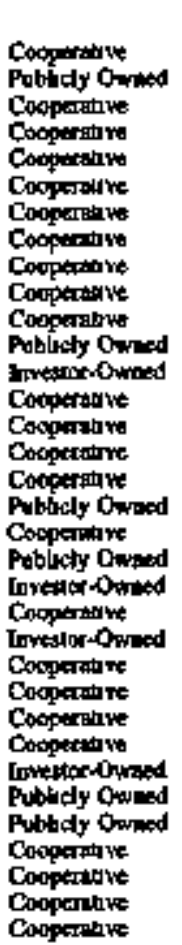 & $\begin{array}{r}42 \\
1 \\
75 \\
4 \\
450 \\
74 \\
43 \\
56 \\
443 \\
382 \\
98 \\
76 \\
342 \\
0 \\
125 \\
60 \\
139 \\
47 \\
226 \\
1,354 \\
31 \\
676 \\
734 \\
160 \\
60 \\
13,429 \\
117 \\
300 \\
3 \\
208 \\
103 \\
3\end{array}$ & $\begin{array}{r}27 \\
5 \\
7 \\
450 \\
1,547 \\
36 \\
69 \\
521 \\
283 \\
98 \\
75 \\
912 \\
2 \\
55 \\
69 \\
311 \\
139 \\
44 \\
410 \\
1,40 \\
109 \\
760 \\
35 \\
42 \\
163 \\
50 \\
11,844 \\
73 \\
552 \\
3 \\
75 \\
58 \\
5\end{array}$ & $\begin{array}{r}30 \\
8 \\
4 \\
450 \\
723 \\
37 \\
65 \\
500 \\
291 \\
101 \\
77 \\
561 \\
2 \\
82 \\
60 \\
432 \\
740 \\
48 \\
341 \\
1360 \\
109 \\
608 \\
25 \\
30 \\
150 \\
60 \\
7332 \\
97 \\
600 \\
3 \\
49 \\
61 \\
5\end{array}$ & $\begin{array}{r}50 \\
1 \\
7 \\
750 \\
727 \\
47 \\
73 \\
536 \\
392 \\
300 \\
85 \\
917 \\
3 \\
70 \\
60 \\
215 \\
143 \\
52 \\
384 \\
0 \\
110 \\
192 \\
25 \\
50 \\
140 \\
43 \\
7,270 \\
82 \\
600 \\
5 \\
44 \\
67 \\
5\end{array}$ \\
\hline
\end{tabular}

Sos tontuotes st eod of table 
Teble 22. U.S. Electric Utlity DSM Program Costs by North Americas Electric Relikiblity Conacil Region and Hawati by Class of Owmership, 1995, 1996, 1997, and 2001 (Thousand Dollars) (Continued)

\begin{tabular}{|c|c|c|c|c|c|}
\hline \multirow{2}{*}{ 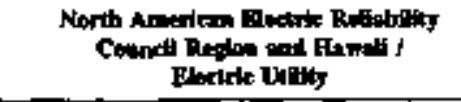 } & \multirow{2}{*}{$\begin{array}{c}\text { Cluw of } \\
\text { Ornershtp }\end{array}$} & \multicolumn{2}{|c|}{ Inopottal Comsts } & \multicolumn{2}{|c|}{ Prejected Com } \\
\hline & & joss & $19 x$ & 507 & 201 \\
\hline 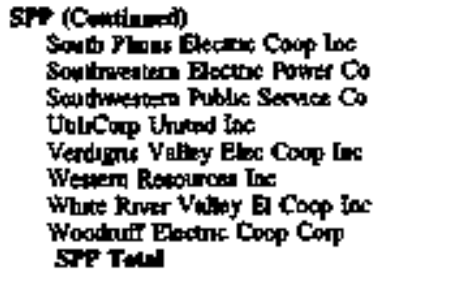 & 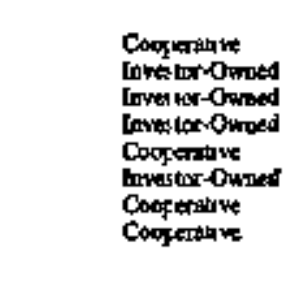 & $\begin{array}{r}534 \\
1,587 \\
2,192 \\
0 \\
122 \\
2,323 \\
7 \\
24,94\end{array}$ & $\begin{array}{r}475 \\
1,479 \\
3,301 \\
0 \\
123 \\
2,352 \\
7 \\
84 \\
2,396\end{array}$ & $\begin{array}{r}525 \\
1,425 \\
1,537 \\
570 \\
128 \\
2,200 \\
8 \\
76 \\
21,007\end{array}$ & $\begin{array}{r}50 \\
0 \\
1.96 \\
570 \\
179 \\
1.776 \\
8 \\
86 \\
1706\end{array}$ \\
\hline 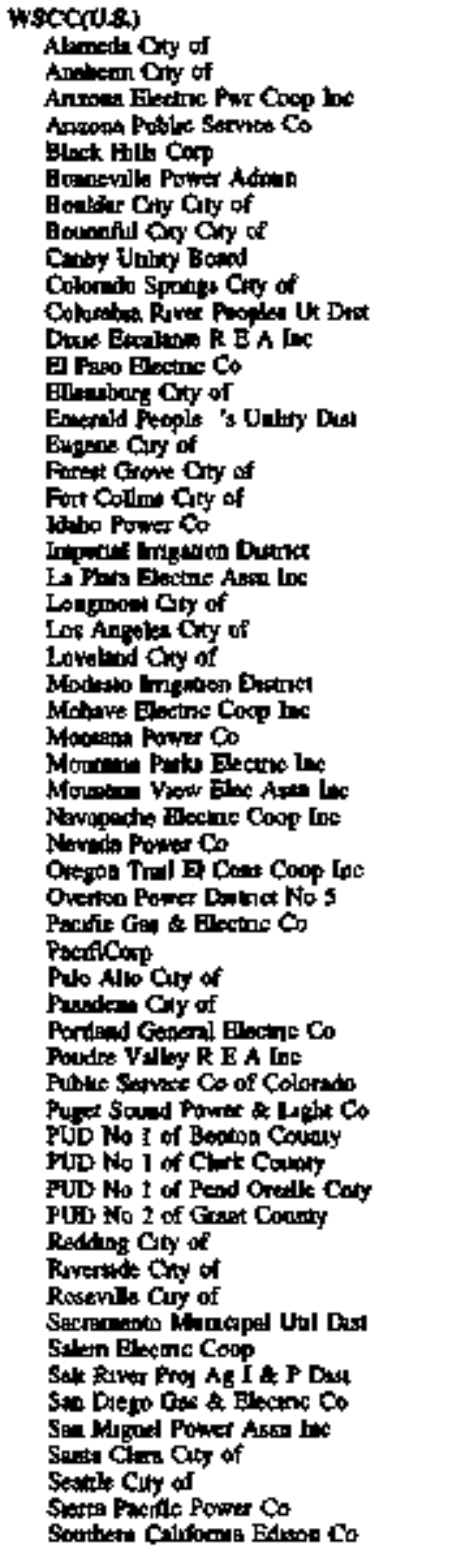 & 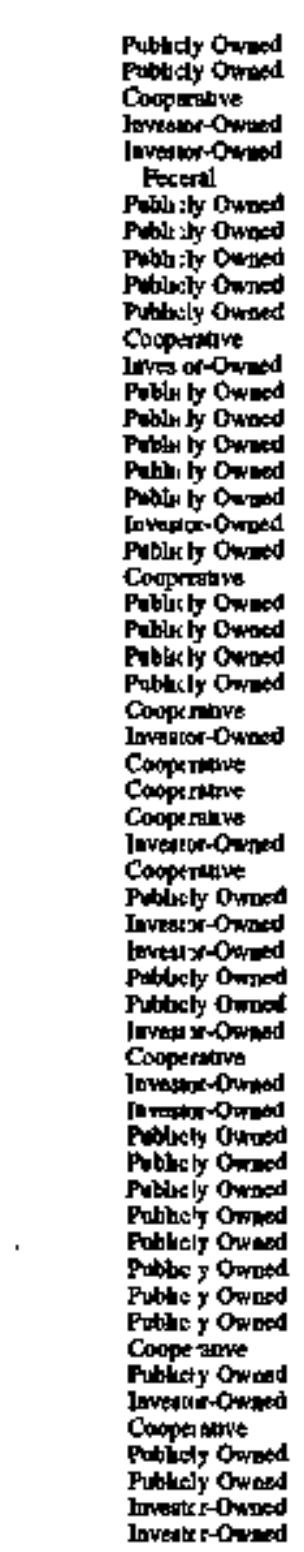 & 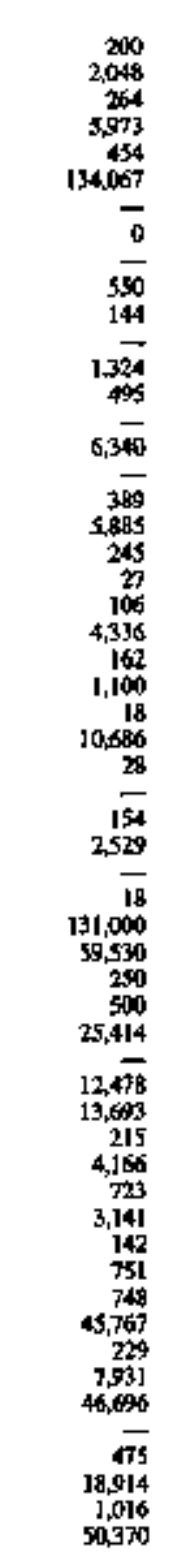 & 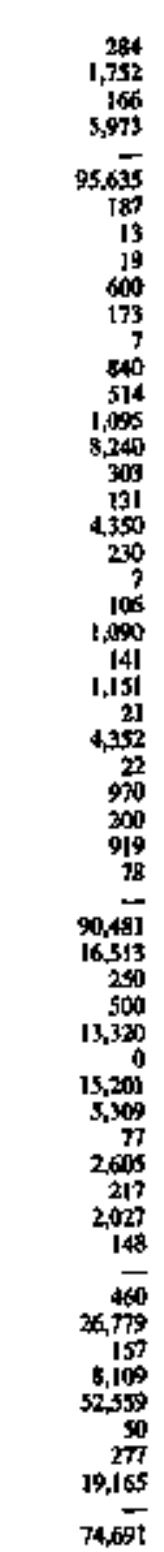 & 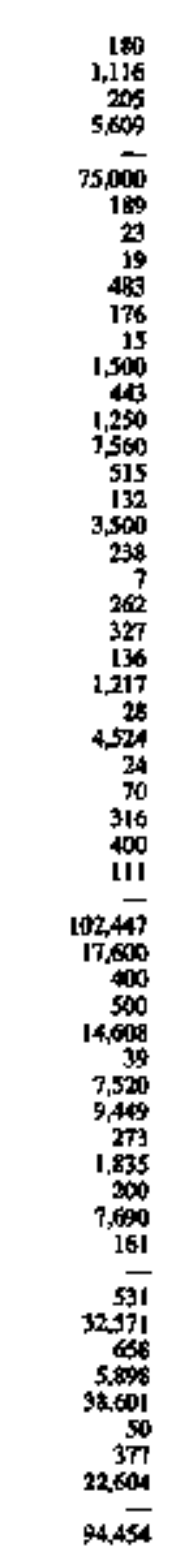 & 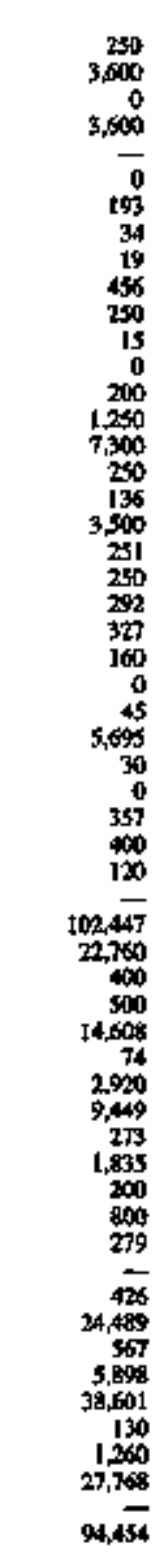 \\
\hline
\end{tabular}

See fortwots at end of labk 
Toble 22. U.S. Electric Utllity ISM Program Costs by North American Electric Reliability Councll Region and Hawel by Class of Ownershlp, 1995, 1996, 1997, and 2001 (Thousand Dollars) (Continued)

\begin{tabular}{|c|c|c|c|c|c|}
\hline \multirow{2}{*}{ 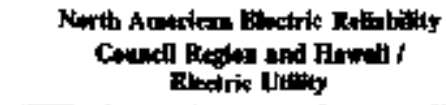 } & \multirow{2}{*}{$\begin{array}{c}\text { Clot of } \\
\text { Omotratip }\end{array}$} & \multicolumn{2}{|c|}{ Iflonien Chin } & \multicolumn{2}{|c|}{ Profected Costs } \\
\hline & & 19ses & $190 \%$ & $\mid s 9)$ & InI \\
\hline 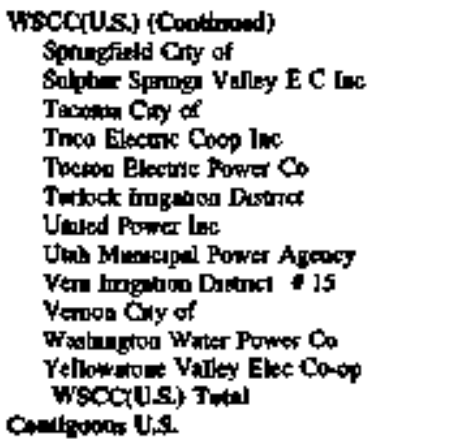 & 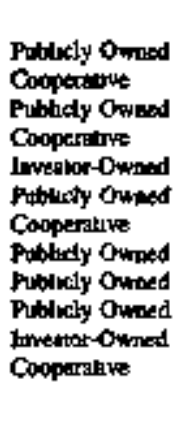 & $\begin{array}{r}2,456 \\
5 \\
7,895 \\
3 \\
3,361 \\
245 \\
97 \\
24 \\
40 \\
65 \\
3,590 \\
194 \\
619,475 \\
2,450,625\end{array}$ & $\begin{array}{r}2,190 \\
5 \\
4,153 \\
3 \\
2,645 \\
245 \\
277 \\
58 \\
\frac{1}{94} \\
3,507 \\
172 \\
17,759 \\
1,4,967\end{array}$ & $\begin{array}{r}2,665 \\
13 \\
7,791 \\
3 \\
2,645 \\
152 \\
470 \\
54 \\
105 \\
4,666 \\
132 \\
4 \$ 2,734 \\
1,960,366\end{array}$ & $\begin{array}{r}1,221 \\
15 \\
4,245 \\
0 \\
2,645 \\
252 \\
230 \\
59 \\
1,157 \\
4,656 \\
150 \\
303,754 \\
1,84,448\end{array}$ \\
\hline 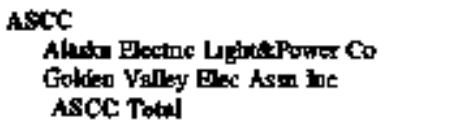 & 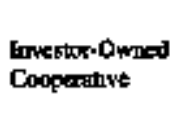 & $\begin{array}{l}121 \\
5: 2 \\
\mathbf{6 5}\end{array}$ & $\begin{array}{r}63 \\
228 \\
21\end{array}$ & $\begin{array}{r}63 \\
277 \\
346\end{array}$ & $\begin{array}{r}78 \\
291 \\
369\end{array}$ \\
\hline 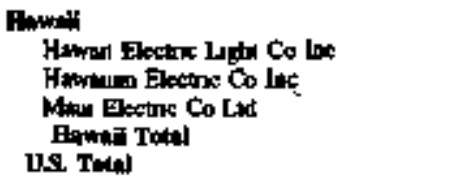 & $\begin{array}{l}\text { lawsicor-Owned } \\
\text { levertor-Owned } \\
\text { Investor-Owed }\end{array}$ & $\begin{array}{r}0 \\
0 \\
0 \\
0 \\
2,41,261\end{array}$ & $\begin{array}{r}1,409 \\
2,404 \\
311 \\
4,124 \\
1,50,167\end{array}$ & $\begin{array}{r}2,254 \\
7,792 \\
2,638 \\
12,644 \\
1,576,34\end{array}$ & $\begin{array}{r}2,0 d 1 \\
0 \\
3,997 \\
5,813 \\
1814297\end{array}$ \\
\hline
\end{tabular}

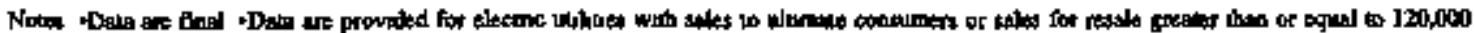

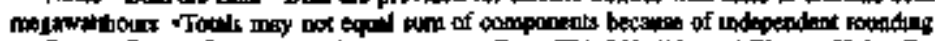

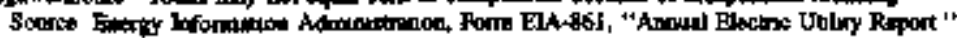


Table 23. U.S. Electrit Uthity DSM Progiram Direct Utillity Costs by North American Flectulc Relinblitty Conncli Region and Hawail by DSM Progran Category, $19 \% 6$ (Thoussand Dollars)

\begin{tabular}{|c|c|c|c|c|c|c|}
\hline 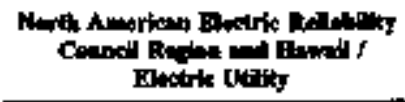 & $\begin{array}{l}\text { Enerdy } \\
\text { Findang }\end{array}$ & $\begin{array}{l}\text { Direat Low } \\
\text { Contrey }\end{array}$ & Intarlathe Loud & Ohar lowd & 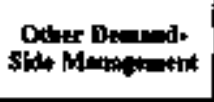 & $\begin{array}{l}\text { Tuent Plreed } \\
\text { Utitty Cows } 1\end{array}$ \\
\hline 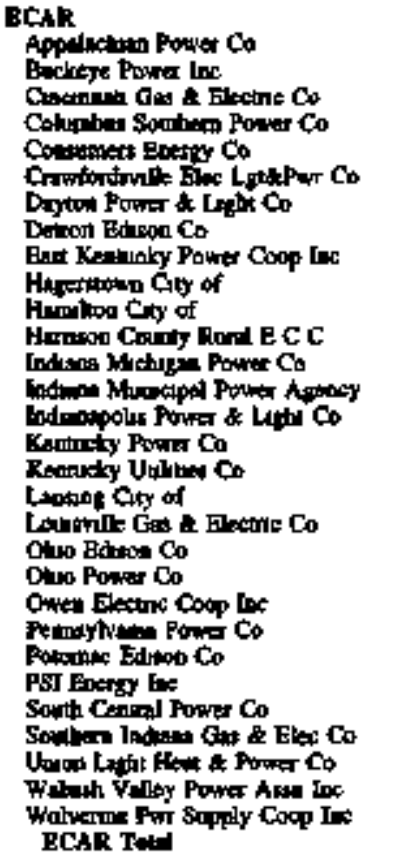 & 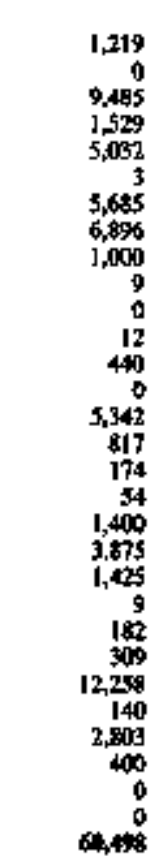 & $\begin{array}{r}1,000 \\
1392 \\
0 \\
1 \\
0 \\
0 \\
10 \\
0 \\
0 \\
0 \\
0 \\
0 \\
377 \\
0 \\
0 \\
4 \\
0 \\
0 \\
0 \\
0 \\
0 \\
0 \\
0 \\
0 \\
0 \\
56 \\
510 \\
1,964 \\
252 \\
240 \\
152 \\
0,145\end{array}$ & $\begin{array}{r}0 \\
0 \\
5 \\
60 \\
0 \\
0 \\
0 \\
0 \\
0 \\
0 \\
0 \\
0 \\
0 \\
0 \\
0 \\
0 \\
1,133 \\
0 \\
0 \\
0 \\
0 \\
0 \\
0 \\
0 \\
0 \\
0 \\
0 \\
0 \\
0 \\
0 \\
0 \\
1,204\end{array}$ & $\begin{array}{r}0 \\
0 \\
0 \\
30 \\
380 \\
0 \\
0 \\
0 \\
0 \\
0 \\
4 \\
18 \\
0 \\
0 \\
0 \\
0 \\
0 \\
0 \\
0 \\
0 \\
1,010 \\
0 \\
0 \\
0 \\
0 \\
0 \\
0 \\
0 \\
0 \\
0 \\
0 \\
0 \\
0 \\
0\end{array}$ & $\begin{array}{r}0 \\
0 \\
0 \\
0 \\
0 \\
0 \\
0 \\
0 \\
0 \\
0 \\
11 \\
0 \\
0 \\
0 \\
0 \\
0 \\
0 \\
0 \\
0 \\
0 \\
0 \\
0 \\
0 \\
\mathbf{0} \\
\mathbf{0} \\
\mathbf{1 6 0} \\
\mathbf{0} \\
\mathbf{0} \\
\mathbf{0} \\
\mathbf{0} \\
\mathbf{1 0}\end{array}$ & 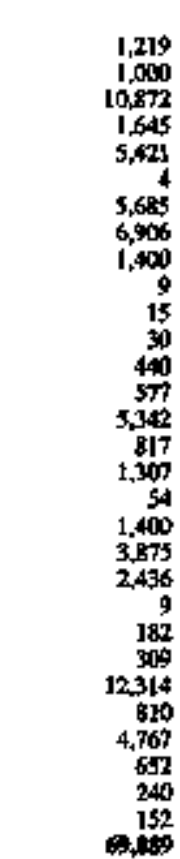 \\
\hline 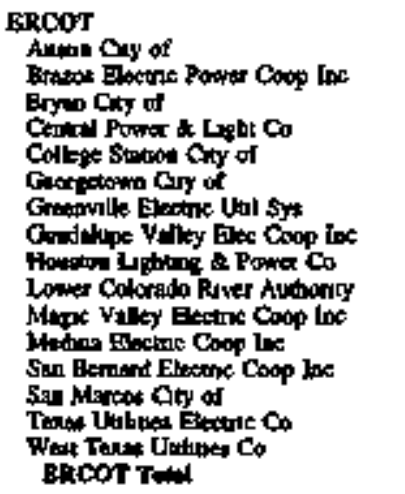 & $\begin{array}{r}10,266 \\
1.167 \\
293 \\
6,766 \\
50 \\
20 \\
10 \\
0 \\
2,855 \\
5,216 \\
125 \\
0 \\
16 \\
22 \\
8,103 \\
5,310 \\
36,200\end{array}$ & $\begin{array}{r}0 \\
0 \\
55 \\
0 \\
0 \\
1 \\
0 \\
4 \\
2,055 \\
0 \\
3,0 \\
0 \\
0 \\
0 \\
0 \\
2,40 \\
2,0\end{array}$ & $\begin{array}{r}0 \\
0 \\
0 \\
0 \\
0 \\
0 \\
10 \\
0 \\
0 \\
332 \\
0 \\
0 \\
45 \\
0 \\
0 \\
0 \\
39\end{array}$ & $\begin{array}{r}0 \\
0 \\
0 \\
0 \\
0 \\
2 \\
0 \\
0 \\
3,92 \\
0 \\
0 \\
29 \\
0 \\
0 \\
92 \\
0 \\
4915\end{array}$ & $\begin{array}{r}0 \\
0 \\
0 \\
0 \\
0 \\
0 \\
0 \\
0 \\
6 \\
0 \\
0 \\
0 \\
0 \\
0 \\
0 \\
0 \\
6\end{array}$ & $\begin{array}{r}10,256 \\
1,167 \\
3,49 \\
6,766 \\
50 \\
28 \\
20 \\
47 \\
3,879 \\
5,548 \\
475 \\
29 \\
61 \\
22 \\
9,065 \\
1,310 \\
4,46\end{array}$ \\
\hline 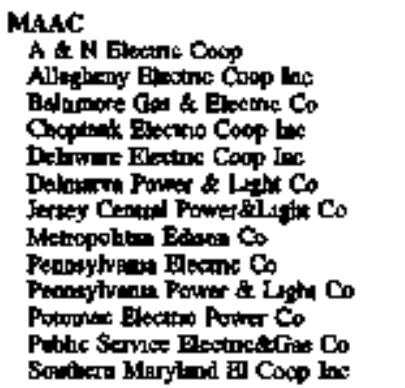 & $\begin{array}{r}0 \\
32 \\
28,752 \\
0 \\
0 \\
3,921 \\
4,672 \\
896 \\
496 \\
9,335 \\
4,251 \\
36,926 \\
3,355\end{array}$ & $\begin{array}{r}143 \\
2657 \\
15201 \\
278 \\
515 \\
4298 \\
3391 \\
0 \\
0 \\
0 \\
11874 \\
7499 \\
3496\end{array}$ & $\begin{array}{r}0 \\
3 \\
4,400 \\
0 \\
0 \\
0 \\
0 \\
0 \\
0 \\
0 \\
1,356 \\
7,329 \\
7\end{array}$ & $\begin{array}{r}0 \\
4 \\
969 \\
0 \\
0 \\
0 \\
0 \\
12 \\
117 \\
0 \\
1,837 \\
0 \\
0\end{array}$ & $\begin{array}{r}0 \\
220 \\
0 \\
0 \\
0 \\
18 \\
0 \\
0 \\
0 \\
0 \\
0 \\
4,729 \\
0\end{array}$ & $\begin{array}{r}143 \\
2,916 \\
6,321 \\
278 \\
515 \\
8,577 \\
8,069 \\
509 \\
573 \\
9,335 \\
60,318 \\
56,477 \\
7,058\end{array}$ \\
\hline
\end{tabular}

Sed fooknotet at tod of mite 
Table 23. U.S. Electric Utility DSM Program Direct Utifty Cost by North American Electric Reliability Coumcil Region and Hawald by DSM Program Categary, 19\% (Thousand Dollars) (Continued)

\begin{tabular}{|c|c|c|c|c|c|c|}
\hline 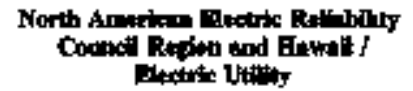 & $\frac{\text { Buerty }}{\text { Dintency }}$ & $\begin{array}{l}\text { Drita Loud } \\
\text { Cominel }\end{array}$ & 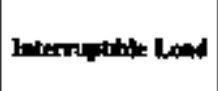 & $\begin{array}{l}\text { Oiner Iand } \\
\text { Minthoutent }\end{array}$ & 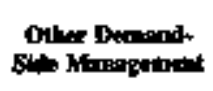 & $\begin{array}{c}\text { Todel Dtreel } \\
\text { Ut ity Cosis } 1\end{array}$ \\
\hline 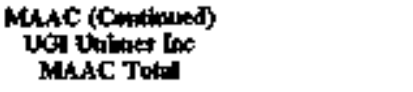 & $133, \frac{61}{67}$ & & 13,114 & 2431 & 4,9 & 204,4 \\
\hline 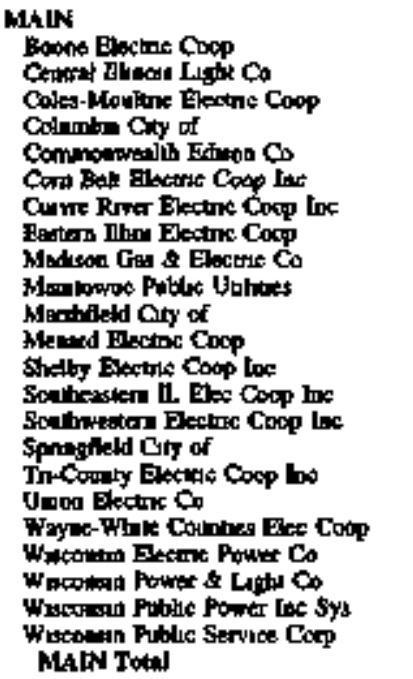 & 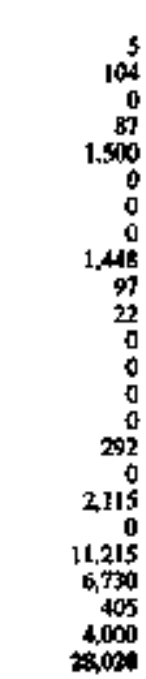 & $\begin{array}{r}85 \\
0 \\
80 \\
397 \\
4,010 \\
0 \\
40 \\
54 \\
295 \\
0 \\
0 \\
99 \\
3 \\
0 \\
38 \\
0 \\
5 \\
198 \\
12 \\
775 \\
92 \\
0 \\
200 \\
6,349\end{array}$ & $\begin{array}{r}1 \\
2,732 \\
0 \\
9 \\
0 \\
0 \\
0 \\
12 \\
0 \\
0 \\
0 \\
7 \\
4 \\
0 \\
0 \\
0 \\
5 \\
10,449 \\
15 \\
10 \\
0 \\
0 \\
3,300 \\
16,744\end{array}$ & $\begin{array}{r}0 \\
0 \\
0 \\
0 \\
3,000 \\
0 \\
0 \\
0 \\
0 \\
0 \\
0 \\
0 \\
6 \\
0 \\
0 \\
0 \\
0 \\
0 \\
0 \\
186 \\
0 \\
0 \\
100 \\
302\end{array}$ & $\begin{array}{r}0 \\
0 \\
0 \\
0 \\
0 \\
160 \\
0 \\
0 \\
0 \\
0 \\
0 \\
0 \\
0 \\
4 \\
0 \\
0 \\
0 \\
0 \\
0 \\
0 \\
9 \\
0 \\
0 \\
173\end{array}$ & $\begin{array}{r}91 \\
2,836 \\
60 \\
453 \\
8,500 \\
160 \\
40 \\
60 \\
1.743 \\
97 \\
22 \\
106 \\
13 \\
4 \\
58 \\
291 \\
10 \\
12,760 \\
77 \\
12,186 \\
6,431 \\
405 \\
7,800 \\
54,501\end{array}$ \\
\hline 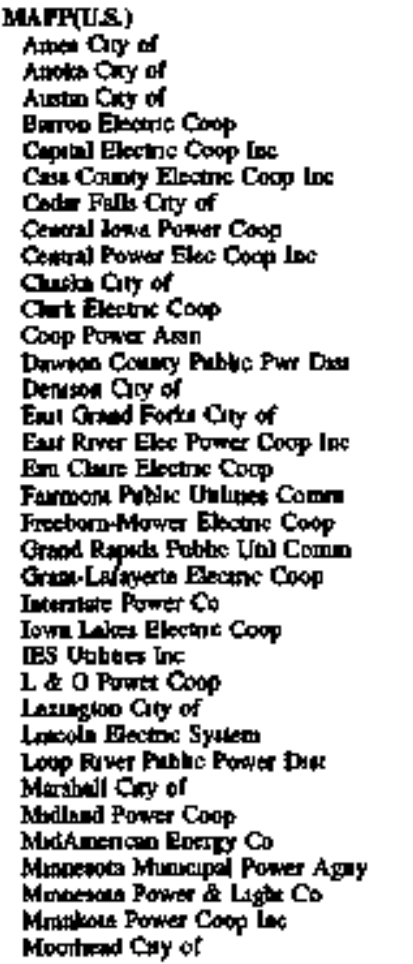 & $\begin{array}{r}10 \\
5 \\
22 \\
6 \\
0 \\
14 \\
390 \\
900 \\
0 \\
105 \\
0 \\
1,165 \\
0 \\
0 \\
100 \\
5588 \\
65 \\
0 \\
50 \\
6 \\
7 \\
3,536 \\
252 \\
8,548 \\
0 \\
0 \\
49 \\
0 \\
2 \\
59 \\
4,592 \\
0 \\
15,591 \\
0 \\
160\end{array}$ & 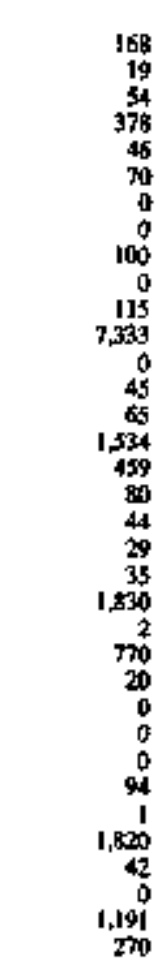 & $\begin{array}{r}0 \\
116 \\
30 \\
0 \\
0 \\
0 \\
0 \\
0 \\
0 \\
0 \\
0 \\
0 \\
12 \\
0 \\
0 \\
0 \\
0 \\
0 \\
0 \\
2 \\
0 \\
20 \\
0 \\
0 \\
0 \\
5 \\
0 \\
26 \\
0 \\
0 \\
60 \\
85 \\
0 \\
0 \\
0 \\
0\end{array}$ & $\begin{array}{c}0 \\
3 \\
0 \\
0 \\
0 \\
0 \\
0 \\
0 \\
0 \\
0 \\
0 \\
0 \\
0 \\
0 \\
0 \\
0 \\
0 \\
0 \\
0 \\
0 \\
0 \\
0 \\
2 \\
18 \\
0 \\
0 \\
8 \\
0 \\
0 \\
0 \\
0 \\
0 \\
0 \\
0 \\
0\end{array}$ & $\begin{array}{r}0 \\
0 \\
0 \\
0 \\
0 \\
0 \\
0 \\
0 \\
0 \\
0 \\
0 \\
50 \\
0 \\
0 \\
31 \\
0 \\
0 \\
24 \\
0 \\
0 \\
0 \\
103 \\
0 \\
0 \\
0 \\
0 \\
0 \\
0 \\
0 \\
0 \\
71 \\
100 \\
0 \\
0 \\
0\end{array}$ & $\begin{array}{r}178 \\
149 \\
106 \\
384 \\
46 \\
84 \\
300 \\
900 \\
100 \\
105 \\
115 \\
9,096 \\
11 \\
45 \\
196 \\
2.99 \\
524 \\
104 \\
54 \\
37 \\
42 \\
5.489 \\
226 \\
9.376 \\
20 \\
5 \\
57 \\
26 \\
96 \\
60 \\
13218 \\
209 \\
15,597 \\
1.191 \\
430\end{array}$ \\
\hline
\end{tabular}

Sow foopotes at and of teiple 
Tahle 23. U.S. Electric Utlity DSM Progeam Direct Uthlity Costs by North American Electric Relibanlity Conneil Reglon and Hawain by DSM Program Category, 1996

(Thousend Dollars) (Continued;

\begin{tabular}{|c|c|c|c|c|c|c|}
\hline 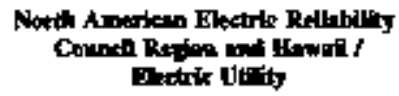 & $\begin{array}{c}\text { Energit } \\
\text { Entelent }\end{array}$ & $\begin{array}{l}\text { Dirnd Lail } \\
\text { Conthol }\end{array}$ & Inimapelble laod & 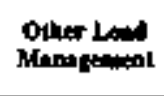 & Othe Dewind. & Tot:l Dunt \\
\hline
\end{tabular}

MAPP(US) (Cantit

Mosmint-Wallew Elac Coop

Mumkapst Energy Apency of ME

Moscanine Crry of

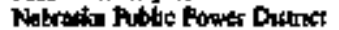

Nadik Elacane Coop be

Nithers Scxtes Pawa Co of MaN

Noalwer sutes Powt $C_{0}$ of WI

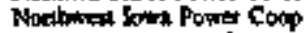

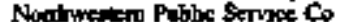

Nonlmoten Wisconon Elac Co

Ouldalo Eveconc Coop.

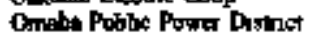

Suke Tal Power Co

Oprotenin Gity of

People 'c Coop Potrot Aard

Puare City of

Polk-Bumet Electre Coop

R S R Electioc Coop In

Red Rurer Yallay Coop Pur Abo

Fice List Uhtures

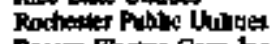

Rower Electuc Cocp be

Sherpec Puble Utilites Coom

Spetcer City of

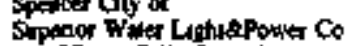

Theof Ruver falts Oty of

Tressealean Electace Conp

Tn-Drin'y Bloctuc Coop

Vined Powner Ast:

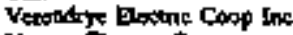

Yauph Elestre Cocp

Yort Donnly Rurl Rb Forr Dss

MthPR(US.) Tatal

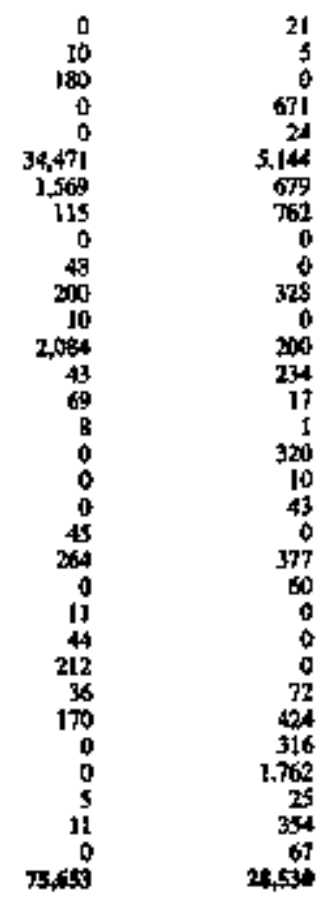

0
0
0
0
0
1223
39
0
2
0
0
0
0
5
0
0
0
0
0
0
0
0
0
0
0
46
0
0
800
25
0
0
916

6
0
0
0
0
0
74
0
0
19
0
0
0
8
0
0
0
0
0
0
0
0
32
0
0
0
0
0
0
5
0
0
030

0
0
0
0
0
0
0
0
0
0
0
0
0
9
0
0
0
0
0
0
0
0
0
0
0
0
0
0
2700
0
0
0
3,6

85
45
180
67
24
40,839
2761
877
2
67
528
10
2,284
308
86
9
320
10
43
45
641
60
65
41
212
154
594
316
5.276
69
364
67
113,472

NTCCOU.S.

Areade Villaye of

Bangox Hydro-Elesterc Co

Btackptome Valley Eectic Co

Basmo Etasen Co

Bravintes Town of

Burlangeres Clyy of

centrigge Dloctar isth to

Corinl Hisdona Ous \& Elec Cocp

Central Mande Pourer Co.

Centrul Vermod Pat Sery Cosp

Coropee Cary of

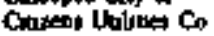

Commorweald Electinc Co

Conord Electinc $\mathrm{Co}$

Cosmothost Luth a Powre Co

Cosancturut Valey Elec Co Ine

Conooldowed Edrood Co-NY he

Exiron Ethom Co

Exear is Hampton Flacenc Do

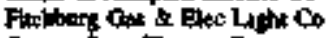

Grante Stwo Electne Co

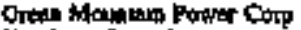

Honphis Cory of

Hosyols Cing of

Inetome Cuy of

Litkina Tows of

Lowe bland Lofertag Co

Mine Rusue Somos of

Mestatuacta Electinc Co

Muta.e Tom of

Prepropiset Electes Co

5
1.162
13.830
65
305
311
1,500
15.706

30

0

5

1.162

13,653

170

305

1,600

16,000

2108

2,100

144

1,248

198

27,

98

38,152

1,987

254

1,694

1,390

20
304
10

15
4
6975

6,975

42,389

0
$0 \leq 50$

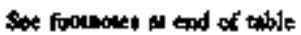


Table 23. U.S. Electric Utility DSM Progtam Direct Utility Costs by North American Electric Reliabillty Conncil Region and Hawal' by DSM Propram Category, 1996 (Thousand Donlars) (Contunued)

\begin{tabular}{|c|c|c|c|c|c|c|}
\hline 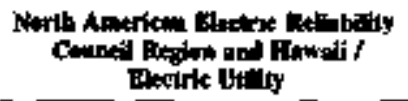 & $\frac{\text { Roprat }}{\text { Eatdery }}$ & $\begin{array}{l}\text { Dinct Loed } \\
\text { Conkint }\end{array}$ & Intertuphple load & $\begin{array}{c}\text { Opher Loed } \\
\text { Mrantrat }\end{array}$ & 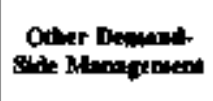 & $\begin{array}{l}\text { Total Dired } \\
\text { Lillty Cacts } 1\end{array}$ \\
\hline
\end{tabular}

Neccr(US) (Corthad)

New Enthed Power Co

Now Harathe Elec Cocp Ite

New Yot Sinc Eloc to Gu Corp

Newpar Elestinc Corp

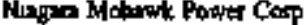

Normoad City of

Oann Inc-

Oripge \& Roctlond Uals Ins

Power Aurinoriry of Scme of NY

Putic Service Co of NH

Returbe Torth of

Rochalar Gas : Electnc Coip

Strowiby Town of

Truncon Cry of

Unued Illontuahe Co

Vorben Elactits Coop Ine

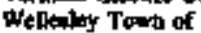

Weater Mrasachosets Biec Co

APCC(US,) Twent

0
689
4,566
539
233
85
1
3,720
8,309
2,532
10
1,107
85
202
5,192
205
0
10,320
205,372

SaRC

Mles Dectinc Coop lie

Alvbinim Eloctric Coop loc

Alneor Mincespat Elac An:t

Alakine Ponver co

Albenards Ciry of

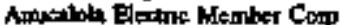

Baiksty Electoc Coop he

Blank River Blecta Cocp lne

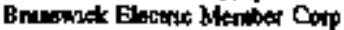

BARC Blectnc Coop Ine

Cinder Cony of

Carolna Power a Ligul Co

Onoll Elecenc Munber Cos

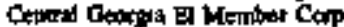

Cootral Yurems Eloctrie Coon

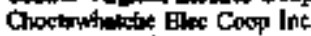

Clay Eloctre Coop Inc

Cobb Electinc Mfamberaty Corp

Colquit Electinc Mombers Corp

Conmbarty Electro Coop

Conela.Fryetue El Master Corp

Conp Courly Former Comp

Deuilat Cor of

Dolo Pour Co

Euky Conbrood Uniny System

Eapl Ponst Chy of

Elzabeth Cxy foy uf

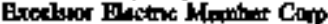

Forfild Dlocme Coep las

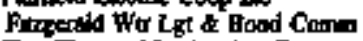

Fin Esctry Minmbettyp Corp

Floade Teys $\mathbf{E}$ Coop Asan Ix

Fand Pante \& Lijo Ca

Fande Pona Corp

Fin Paxe Untho Auth

Gurevilk Repoon Uthbes:

Ferrit Porer $\mathrm{Co}$

Grots Conuty Blac Member Corp

Groemlk Ublinas Comn

Groe Comn of Pable Works

Groysoms Power Com

Chit Power Co

Harntobabur Caly of

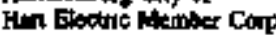

Hoywod Eledte Manibe Corp

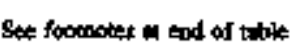

6

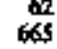

$\begin{array}{rr}853 & 5,233 \\ 257 & 0 \\ 0 & 0 \\ 0 & 0 \\ 0 & 0 \\ 13 & 0 \\ 0 & 0 \\ 0 & 1,739 \\ 0 & 0 \\ 0 & 0 \\ 15 & 50 \\ 0 & 0 \\ 20 & 0 \\ 0 & 0 \\ 0 & 0 \\ 0 & 0 \\ 0 & 0 \\ 0 & 0 \\ 1.19 & 0\end{array}$

0
0
0
0
0
7
0
0
0
0
0
0
0
0
38
0
18
0
0

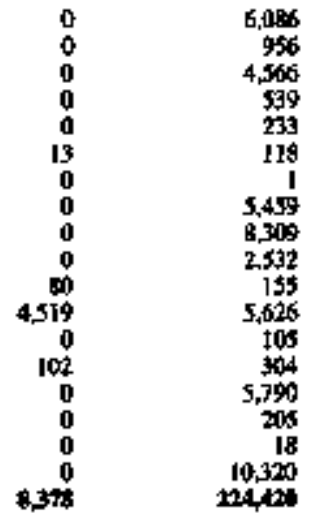

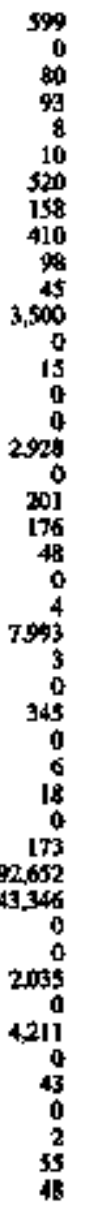<smiles>CCC</smiles>

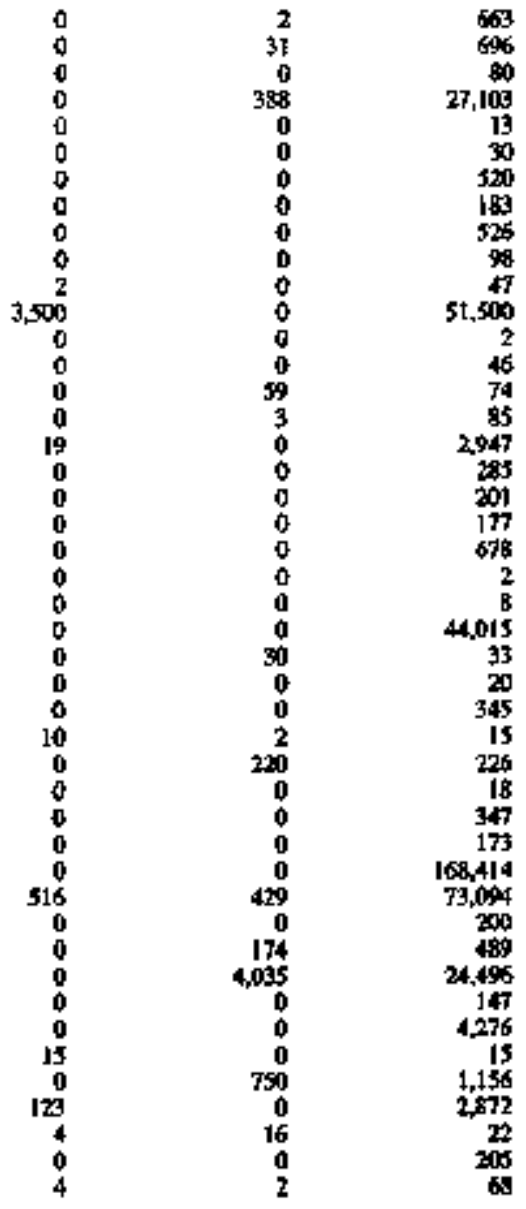




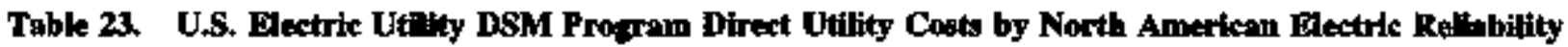
Conncll Region and Hawati by DSM Progaran Cotegory, 19\%6

(Thousand Dollars) (Continucd)

\begin{tabular}{|c|c|c|c|c|c|c|}
\hline 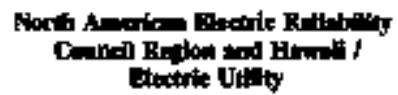 & $\begin{array}{l}\text { Eumpras } \\
\text { Eatedeg }\end{array}$ & Dirtet Lond & Intumptila Ianda & Oilne toud & 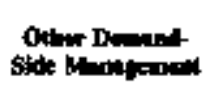 & $\begin{array}{l}\text { Tetel Dtret } \\
\text { utth Cots I }\end{array}$ \\
\hline
\end{tabular}

St:sce (Continad)

Hich Point Tow of

Jectaod Elextric Meriber Corp...........

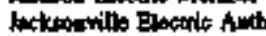

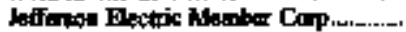

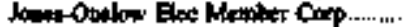

Rinand Ghy of,

Latelol Cory of

Interts Electric Coop in

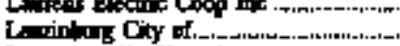

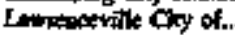

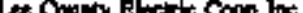

Lothelop City of

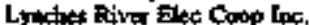

Migursess Ciny of.

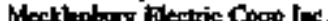

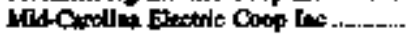

Motisapuic Fonis Co

Mitelen Blectele Whater Corp............

Nor gad Cxy of.

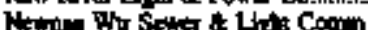

Nonth Comal'se Eusum M P A .

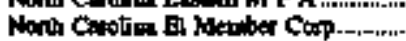

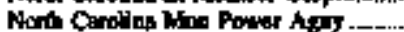

Nathar Nack 2loc Conp In

Mentera Mrdinin Elec Corp................

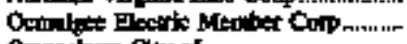

Oringersers Chy of

Oulndo thaties Conn

Falone Eimate Coop

Pes Des Fuctic Cocp lo

Prince Deore Dectric Coop

Rappotinente Electric Coeg.

Pryle Eloctic Memberip Cot ........

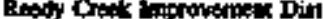

Rock Hid Cing of

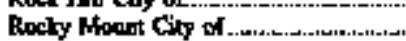

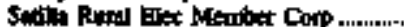

Smod Elzetric Mamber Cop .-_....-

Shentalod Valey Ele Cotp

Stinging Rive Elec Powt At:

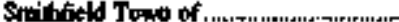

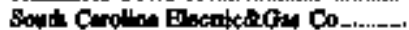

Sinth Cuting Pab Sor Anth u.........

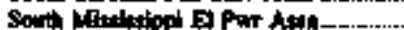

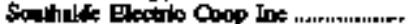

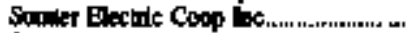

Summer Valky Dex Coop lac ..........

Tallehwese City of

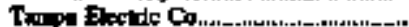

Tamesteve Volley Autionity ..................

Ithoreville City of.

Thlolend Enoric Menter Coip

Triconty Eec Merbe Com

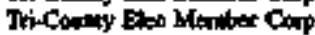

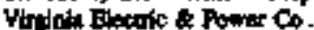

wing hing of

Wilog Fry of

Wintrooche River Elec Coop.........

S:3C THal

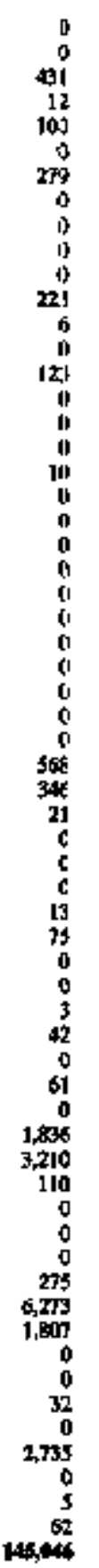

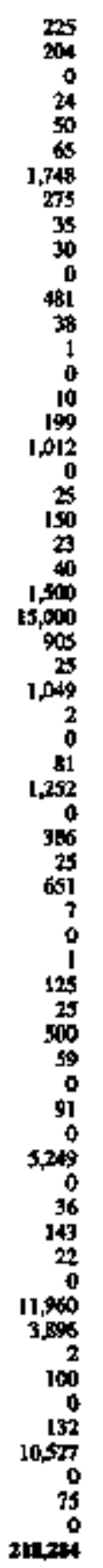

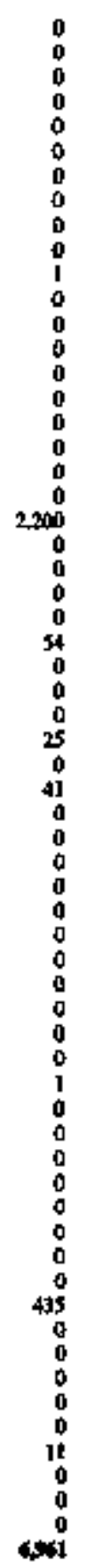

225

204

431
42

160

1,677

2,027

275

30

2

716

I

21

10

1002

10

2

2,350

40

1,575

IS,000

959

$2,2,21$

is

67

1,646

45
396

25

651

20

28

125

28

99

52

1,836
1,499

110

151

151
23

606

$\mathbf{1} 8668$

5,945

2

$\begin{array}{r}32 \\ +42 \\ \hline\end{array}$

2.,542

sintis

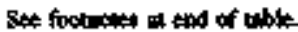


Tabfe 23. U.S. Electric Utility DSM Program Direct Utlity Costs by North American Electrle Rellibility Council Region and Howall by DSM Program Catezary, 19\% (Thousand Dollars) (Contimued)

\begin{tabular}{|c|c|c|c|c|c|c|}
\hline 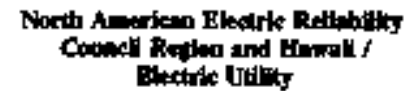 & 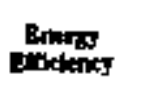 & 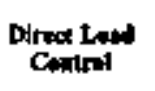 & Intennptilla Isat & Oter Loed & 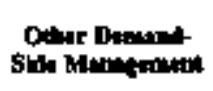 & $\begin{array}{l}\text { Totel Diruat } \\
\text { Dethity Cust] ] }\end{array}$ \\
\hline 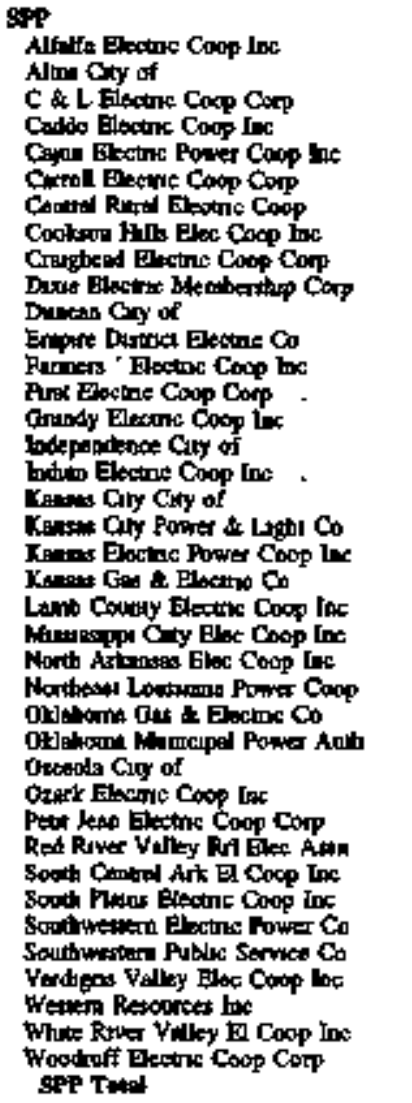 & 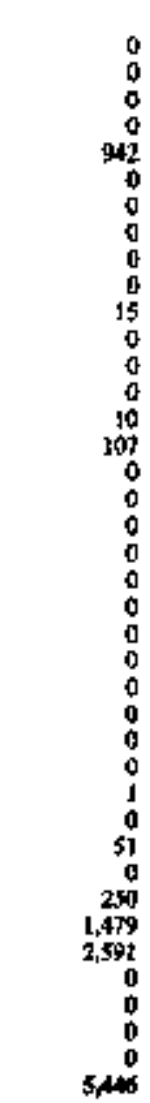 & 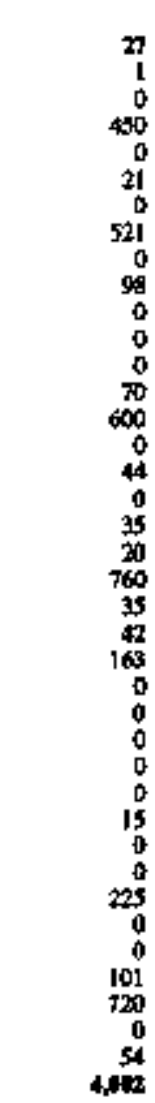 & 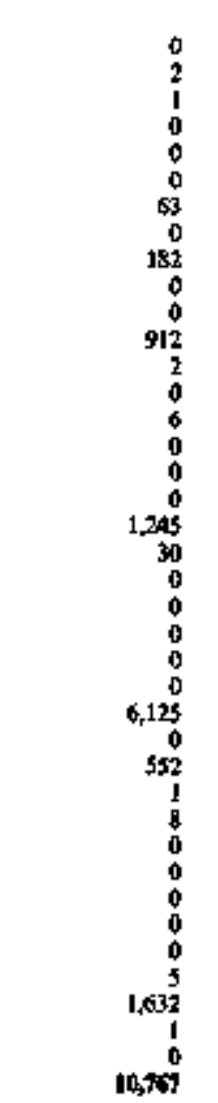 & $\begin{array}{l}0 \\
0 \\
0 \\
0 \\
0 \\
0 \\
0 \\
0 \\
0 \\
0 \\
0 \\
0 \\
0 \\
0 \\
0 \\
0 \\
0 \\
0 \\
0 \\
2 \\
0 \\
0 \\
0 \\
0 \\
0 \\
0 \\
0 \\
0 \\
0 \\
0 \\
0 \\
\mathbf{1} \\
0 \\
0 \\
0 \\
0 \\
0 \\
0 \\
20 \\
20\end{array}$ & $\begin{array}{r}0 \\
0 \\
0 \\
0 \\
0 \\
0 \\
0 \\
0 \\
0 \\
0 \\
0 \\
0 \\
0 \\
0 \\
0 \\
0 \\
0 \\
19 \\
0 \\
12 \\
0 \\
0 \\
0 \\
0 \\
50 \\
5,719 \\
41 \\
0 \\
0 \\
0 \\
3 \\
0 \\
0 \\
0 \\
0 \\
0 \\
0 \\
0 \\
0 \\
0 \\
0 \\
0 \\
0 \\
0 \\
0 \\
0 \\
0\end{array}$ & $\begin{array}{r}27 \\
3 \\
1 \\
450 \\
942 \\
2] \\
63 \\
521 \\
182 \\
95 \\
15 \\
912 \\
2 \\
70 \\
616 \\
107 \\
44 \\
19 \\
1,280 \\
64 \\
760 \\
35 \\
42 \\
163 \\
50 \\
11+844 \\
41 \\
552 \\
2 \\
23 \\
54 \\
4 \\
475 \\
1,479 \\
2591 \\
106 \\
2352 \\
1 \\
74 \\
205\end{array}$ \\
\hline 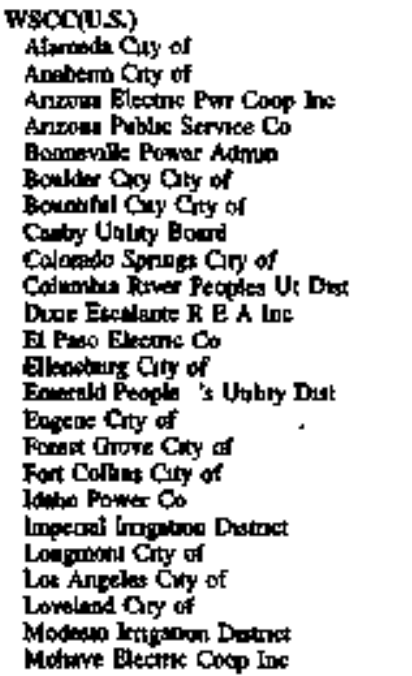 & $\begin{array}{r}142 \\
493 \\
0 \\
3,135 \\
64,075 \\
105 \\
4 \\
16 \\
300 \\
36 \\
0 \\
375 \\
410 \\
1,095 \\
5,700 \\
2588 \\
0 \\
3,741 \\
109 \\
7 \\
678 \\
90 \\
461 \\
3\end{array}$ & $\begin{array}{r}0 \\
2 \\
166 \\
0 \\
0 \\
0 \\
0 \\
0 \\
0 \\
0 \\
0 \\
0 \\
0 \\
0 \\
0 \\
0 \\
100 \\
0 \\
0 \\
0 \\
0 \\
0 \\
271 \\
3\end{array}$ & $\begin{array}{r}18 \\
+26 \\
0 \\
0 \\
0 \\
0 \\
7 \\
0 \\
0 \\
0 \\
2 \\
0 \\
0 \\
0 \\
0 \\
0 \\
0 \\
0 \\
0 \\
0 \\
0 \\
0 \\
419 \\
0\end{array}$ & $\begin{array}{r}0 \\
330 \\
0 \\
0 \\
0 \\
0 \\
0 \\
0 \\
0 \\
0 \\
0 \\
100 \\
0 \\
0 \\
0 \\
0 \\
0 \\
0 \\
0 \\
0 \\
0 \\
6 \\
0 \\
0\end{array}$ & $\begin{array}{r}0 \\
170 \\
0 \\
0 \\
6,695 \\
0 \\
0 \\
0 \\
0 \\
0 \\
0 \\
0 \\
0 \\
0 \\
0 \\
0 \\
0 \\
0 \\
0 \\
7 \\
0 \\
0 \\
0 \\
0\end{array}$ & $\begin{array}{r}160 \\
5.241 \\
166 \\
3,135 \\
30,770 \\
105 \\
11 \\
16 \\
500 \\
36 \\
2 \\
475 \\
410 \\
1,099 \\
5,700 \\
258 \\
101 \\
3,741 \\
189 \\
14 \\
678 \\
96 \\
1,151 \\
6\end{array}$ \\
\hline
\end{tabular}

Sec footinoke at ead of uble 
Table 23. USS. Electric Utility DSM Program Direct Utility Costs by North American Electric Reliability Conucis Ragion and Hawai by DSM Propram Category, 1996

(Thousand Dollars) (Contpums)

\begin{tabular}{|c|c|c|c|c|c|c|}
\hline 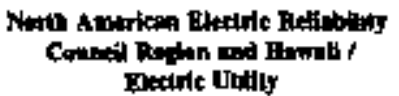 & 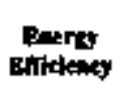 & $\begin{array}{l}\text { Dreet Lood } \\
\text { Conirol }\end{array}$ & 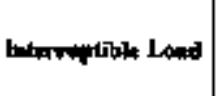 & 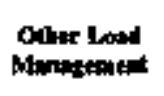 & 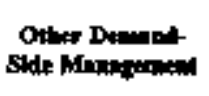 & $\begin{array}{l}\text { Tosor Dintt: } \\
\text { Dellyy Coda } 1\end{array}$ \\
\hline
\end{tabular}

\section{WSCCDS, (Conilment) \\ Montions Power Co \\ Mountin View Elec Aten Ine

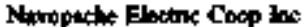 \\ Nevecom tower $C_{0}$ \\ Orton Trut El Cose Coop lo

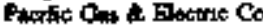 \\ Pacticonp \\ Pab alo Cxy of \\ Portland Geoerl Hection Co \\ Puble Servite Co of Colcrads \\ Pases Sound Power \& Liptr Co \\ PIJ No 1 of Redua Coanty \\ PUD N I of Cort County \\ PUD tho 1 of Rasd Oselle Coty \\ FetD N 2 of Grant County \\ Redting Coy of \\ Rosende Cuy of \\ Secramento Kulunctpal Ub] Deat \\ Salem Dlecter Cocp \\ Salu Rever Proy At I \& P Der \\ Som Deap Gas \& Eloctox Co \\ San Magual Fower Aata lac \\ Sunti Cart Giry of \\ Seatie Cry of

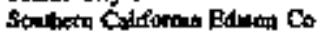 \\ Springiold Dity of \\ 5upher Sprope Valky E C lac \\ Tomoene Gry of \\ Tneo Eectec Corp the \\ Taceor Eletrs Power Co \\ Triack Impronon Dident \\ Uarted Powar Exe \\ Unh Mhmecpel Pown Agency \\ Yernon City of \\ Wentingtos Wimer Power Co \\ Yellowitone Valey Glec Co-op \\ wscc(us.) TH \\ Cwrikgeu US.}

\section{$\mathrm{ABCC}$}

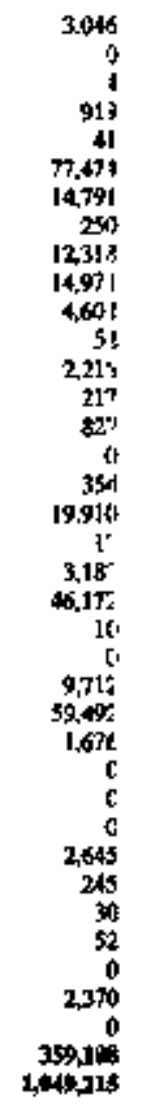

0
700
65
0
0
0
0
0
0
0
0
0
0
0
0
24
75
2,447
0
0
0
25
0
0
576
0
5
1,318
0
0
0
52
0
0
0
0
596
32589

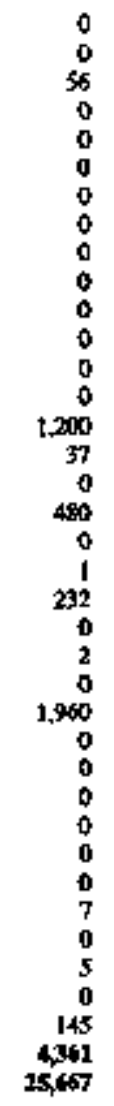

\begin{tabular}{|c|c|}
\hline 0 & 3,046 \\
\hline 0 & 800 \\
\hline 21 & 149 \\
\hline 0 & 919 \\
\hline 0 & 41 \\
\hline 0 & 78,738 \\
\hline 0 & 14,791 \\
\hline 0 & 250 \\
\hline 0 & 12,318 \\
\hline 0 & $\mid 5,021$ \\
\hline a & 4,602 \\
\hline 0 & 52 \\
\hline 0 & 2,215 \\
\hline 0 & 217 \\
\hline 0 & 2007 \\
\hline$\pi$ & 148 \\
\hline 0 & 429 \\
\hline 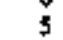 & 21884 \\
\hline 0 & if \\
\hline 0 & 3,IBS \\
\hline I & 46,600 \\
\hline 0 & 35 \\
\hline D & 202 \\
\hline 0 & 9.712 \\
\hline 0 & 62,843 \\
\hline 42 & 1,718 \\
\hline 0 & \\
\hline 0 & 1,318 \\
\hline 0 & \\
\hline 0 & 2645 \\
\hline 0 & 245 \\
\hline 0 & 95 \\
\hline 0 & 52 \\
\hline II & 16 \\
\hline o & 2,370 \\
\hline 0 & 145 \\
\hline 7,032 & 37,409 \\
\hline & $1,620,4+7$ \\
\hline
\end{tabular}

0
0
56
0
0
0
0
0
0
0
0
0
0
0
200
37
0
480
0
1
292
0
2
0
960
0
0
0
0
0
0
7
0
5
0
145
401
467

200

41

250

4,602

52

217

429

IBS

292

,TI8

318

63

245

95

16

145

$1,62 x, 417$

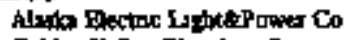
Golden Vaney Elac Assa Inc

ASCC T너미

\footnotetext{
Hown

Hewa Electex Lali co lnc

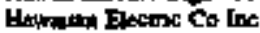

Man Elestac Co Lol

Kinds Thol

U.S. Total
}

12

tiss

4

14
0
14

1,409
852
311
2,502
$1,051,92$

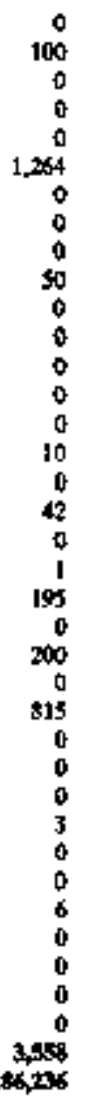

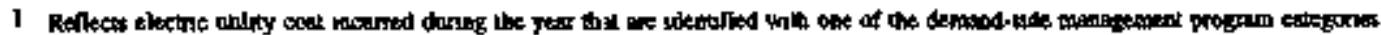

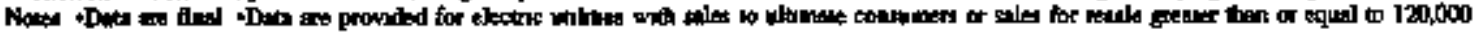
metawionotion

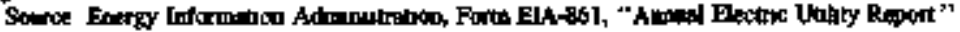


Table 24. U.S. Electric Utility DSM Progran Indirect Utility Costs by North American Electric Rellabiaty Council Rezion and Bawall by Cost Category, 1996 (Thousand Dotlars)

\begin{tabular}{|c|c|c|c|c|c|}
\hline 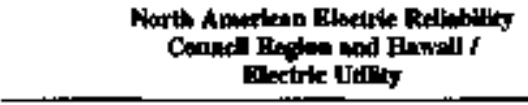 & MtitidndFe & Mertifins & $\begin{array}{l}\text { Menitorlyt } \\
\text { Ind } \\
\text { Drition }\end{array}$ & Denr I & 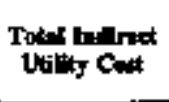 \\
\hline 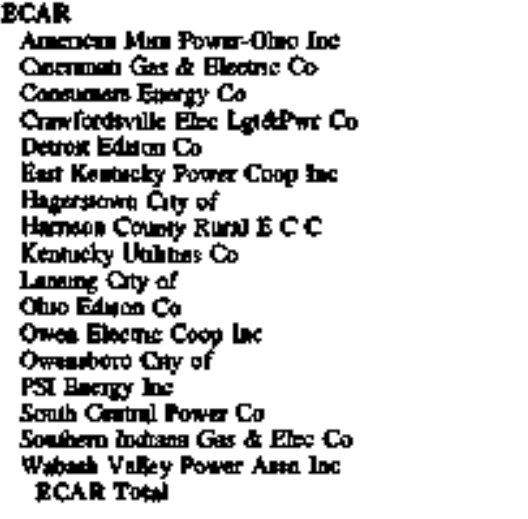 & $\begin{array}{r}14 \\
145 \\
370 \\
1 \\
255 \\
400 \\
0 \\
6 \\
274 \\
0 \\
261 \\
0 \\
0 \\
97 \\
20 \\
450 \\
\mathbf{9 0} \\
2,723\end{array}$ & $\begin{array}{r}0 \\
2 \\
0 \\
0 \\
0 \\
100 \\
10 \\
0 \\
1.451 \\
15 \\
0 \\
43 \\
0 \\
8 \\
15 \\
400 \\
10 \\
2,044\end{array}$ & $\begin{array}{r}5 \\
2 \\
118 \\
0 \\
59 \\
150 \\
0 \\
0 \\
92 \\
2 \\
100 \\
0 \\
0 \\
6 \\
0 \\
452 \\
25 \\
1495\end{array}$ & $\begin{array}{r}25 \\
169 \\
0 \\
0 \\
0 \\
0 \\
0 \\
0 \\
0 \\
0 \\
0 \\
0 \\
25 \\
551 \\
0 \\
12 \\
75 \\
16\end{array}$ & $\begin{array}{r}51 \\
318 \\
488 \\
1 \\
794 \\
650 \\
10 \\
6 \\
1,827 \\
17 \\
161 \\
43 \\
23 \\
1,042 \\
35 \\
1,314 \\
160 \\
7,142\end{array}$ \\
\hline 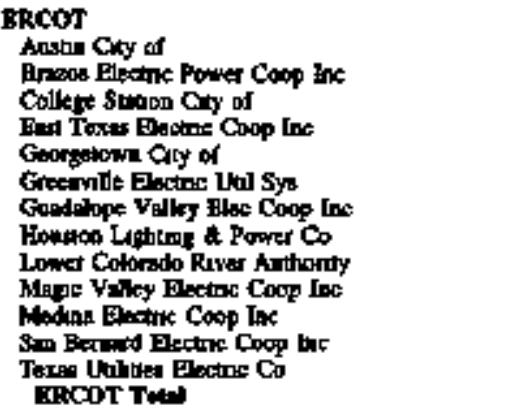 & $\begin{array}{r}1.465 \\
76 \\
50 \\
0 \\
5 \\
8 \\
7 \\
2.472 \\
644 \\
25 \\
15 \\
4 \\
598\end{array}$ & $\begin{array}{r}690 \\
0 \\
8 \\
0 \\
0 \\
4 \\
1 \\
795 \\
0 \\
3 \\
0 \\
0 \\
0 \\
1,50\end{array}$ & $\begin{array}{r}573 \\
0 \\
0 \\
0 \\
10 \\
3 \\
35 \\
108 \\
0 \\
10 \\
3 \\
0 \\
0 \\
942\end{array}$ & $\begin{array}{r}0 \\
0 \\
0 \\
\mathbf{0} \\
0 \\
0 \\
0 \\
0 \\
2,3 \pi \\
0 \\
0 \\
0 \\
0 \\
0 \\
0 \\
1,40\end{array}$ & $\begin{array}{r}2.728 \\
76 \\
58 \\
80 \\
15 \\
15 \\
43 \\
5,736 \\
604 \\
38 \\
18 \\
4 \\
589 \\
5,44\end{array}$ \\
\hline 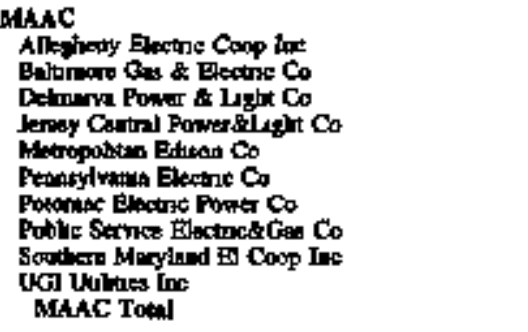 & $\begin{array}{r}472 \\
2111 \\
0 \\
2,078 \\
1.395 \\
955 \\
2.45 \\
1,007 \\
1.18 \\
21 \\
10,062\end{array}$ & $\begin{array}{r}\$ 9 t \\
0 \\
1,020 \\
1,376 \\
5 \$ 9 \\
994 \\
642 \\
648 \\
9] \\
00 \\
5,151\end{array}$ & $\begin{array}{r}6 \\
520 \\
336 \\
77 \\
0 \\
0 \\
13 \\
0 \\
0 \\
21 \\
975\end{array}$ & $\begin{array}{r}4 \\
0 \\
0 \\
1,609 \\
1,466 \\
1,305 \\
0 \\
0 \\
0 \\
0 \\
4,39\end{array}$ & $\begin{array}{r}873 \\
2,691 \\
1,354 \\
5.136 \\
3,450 \\
2,654 \\
3,140 \\
1,075 \\
209 \\
40 \\
31,176\end{array}$ \\
\hline 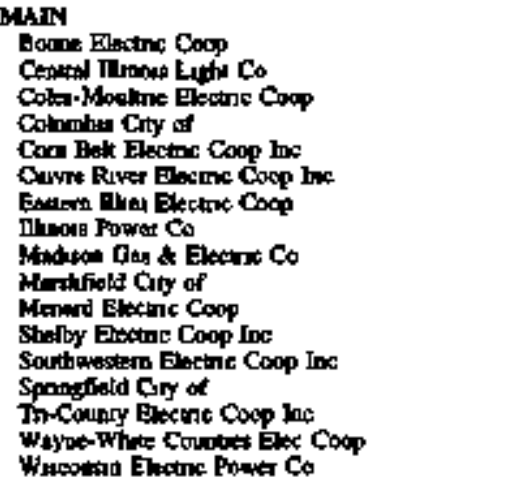 & $\begin{array}{r}151 \\
0 \\
0 \\
29 \\
9 \\
0 \\
0 \\
0 \\
1,799 \\
23 \\
2 \\
4 \\
88 \\
63 \\
2 \\
4 \\
5,744\end{array}$ & $\begin{array}{r}2 \\
0 \\
50 \\
88 \\
8 \\
1 \\
10 \\
10 \\
0 \\
677 \\
42 \\
8 \\
2 \\
2 \\
10 \\
117 \\
2 \\
0 \\
934\end{array}$ & $\begin{array}{r}1 \\
0 \\
0 \\
10 \\
0 \\
4 \\
20 \\
0 \\
177 \\
21 \\
6 \\
4 \\
0 \\
15 \\
0 \\
2 \\
206\end{array}$ & $\begin{array}{c}0 \\
0 \\
0 \\
0 \\
0 \\
0 \\
0 \\
0 \\
0 \\
26 \\
0 \\
0 \\
0 \\
0 \\
0 \\
0 \\
0\end{array}$ & $\begin{array}{r}5 \\
151 \\
50 \\
381 \\
17 \\
5 \\
30 \\
1 \\
2613 \\
212 \\
16 \\
10 \\
98 \\
195 \\
4 \\
6 \\
0,974\end{array}$ \\
\hline
\end{tabular}

Sto formosed at end of lable 
Table 24. U.S. Electric Utilty DSM Program Indirect Ut"lity Coubs by North American Electric Refiabilty Comeil Region and Hewat by Cost Catepory, 1996 (Thousand Dollars) (Contínued)

\begin{tabular}{|c|c|c|c|c|c|}
\hline 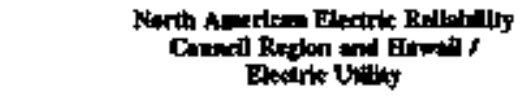 & 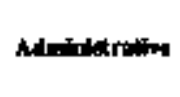 & Mirteilng & Bupltoring & Dater I & 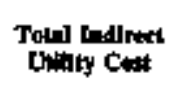 \\
\hline 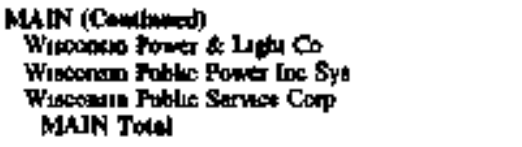 & 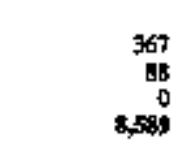 & $\begin{array}{r}0 \\
0 \\
3,500 \\
5,451\end{array}$ & $\begin{array}{r}1,149 \\
0 \\
0 \\
t, 715\end{array}$ & $\begin{array}{r}0 \\
0 \\
0 \\
7\end{array}$ & $\begin{array}{r}1,516 \\
888 \\
3,500 \\
15,72\end{array}$ \\
\hline 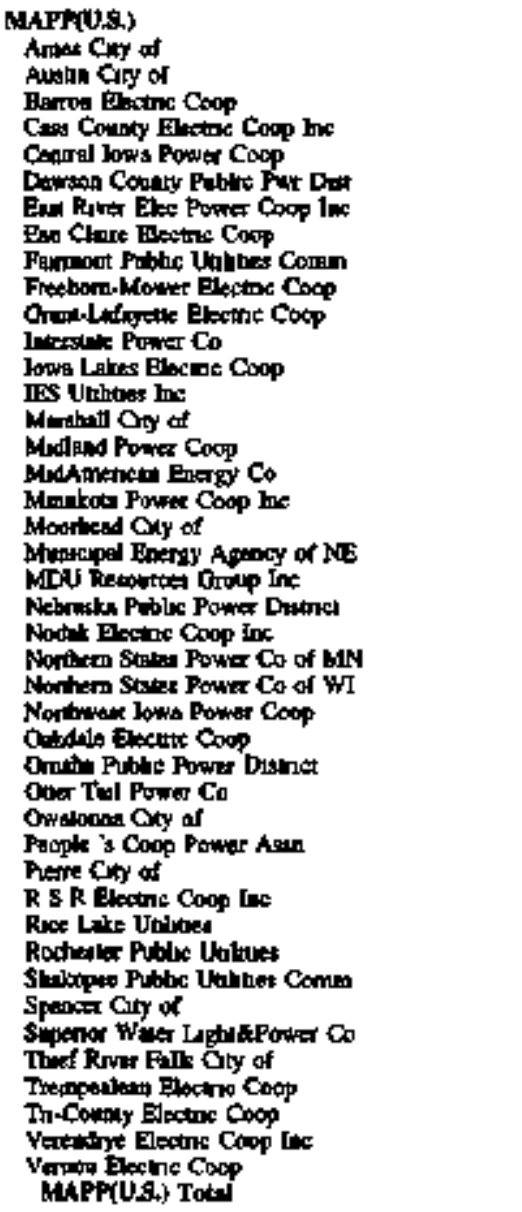 & $\begin{array}{r}52 \\
32 \\
4 \\
225 \\
0 \\
0 \\
0 \\
0 \\
27 \\
228 \\
26 \\
1,141 \\
11 \\
12 \\
654 \\
50 \\
81 \\
15 \\
341 \\
151 \\
11 \\
17,909 \\
106 \\
10 \\
40 \\
50 \\
0 \\
12 \\
0 \\
1 \\
10 \\
17 \\
40 \\
1 \\
4 \\
149 \\
9 \\
0 \\
30 \\
10 \\
0 \\
21,41\end{array}$ & 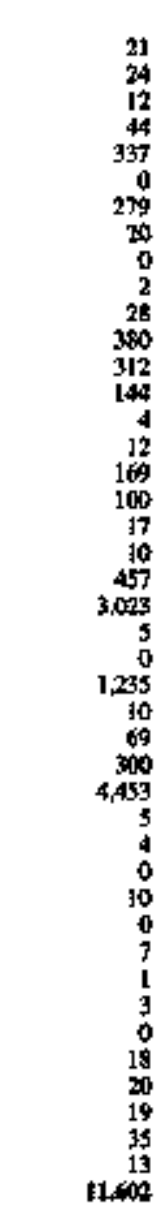 & $\begin{array}{r}0 \\
8 \\
0 \\
4 \\
112 \\
0 \\
0 \\
0 \\
0 \\
0 \\
10 \\
234 \\
21 \\
1910 \\
1 \\
4 \\
198 \\
0 \\
0 \\
5 \\
0 \\
5 \\
35 \\
0 \\
203 \\
5 \\
0 \\
0 \\
0 \\
2 \\
0 \\
1 \\
2 \\
0 \\
3 \\
0 \\
2 \\
0 \\
0 \\
0 \\
0 \\
5 \\
0 \\
2063\end{array}$ & $\begin{array}{r}0 \\
0 \\
0 \\
0 \\
0 \\
10 \\
0 \\
0 \\
0 \\
0 \\
0 \\
0 \\
0 \\
1,490 \\
0 \\
0 \\
\mathbf{1}, \mathbf{6 5} \\
0 \\
0 \\
0 \\
0 \\
0 \\
0 \\
0 \\
0 \\
0 \\
0 \\
0 \\
0 \\
0 \\
0 \\
0 \\
0 \\
0 \\
0 \\
0 \\
0 \\
0 \\
0 \\
0 \\
3 \\
0 \\
0 \\
0 \\
0 \\
0 \\
3 \\
0 \\
3,110\end{array}$ & $\begin{array}{r}79 \\
61 \\
12 \\
52 \\
674 \\
10 \\
279 \\
20 \\
1 \\
65 \\
842 \\
359 \\
4,644 \\
16 \\
28 \\
2,678 \\
130 \\
98 \\
30 \\
801 \\
3,155 \\
54 \\
17,909 \\
1,634 \\
25 \\
499 \\
390 \\
4,453 \\
19 \\
4 \\
2 \\
22 \\
17 \\
50 \\
2 \\
12 \\
119 \\
27 \\
20 \\
49 \\
53 \\
13 \\
39,440\end{array}$ \\
\hline 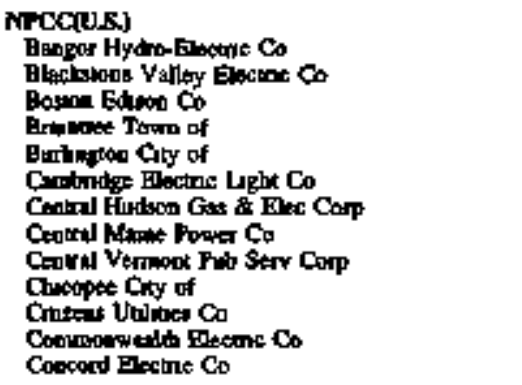 & $\begin{array}{r}42 \\
294 \\
732 \\
30 \\
114 \\
228 \\
44 \\
563 \\
1,153 \\
10 \\
814 \\
382 \\
129\end{array}$ & $\begin{array}{r}0 \\
67 \\
0 \\
3 \\
1 \\
0 \\
0 \\
0 \\
0 \\
8 \\
251 \\
0 \\
1\end{array}$ & $\begin{array}{r}0 \\
57 \\
1,303 \\
0 \\
44 \\
25 \\
70 \\
0 \\
77 \\
2 \\
9 \\
65 \\
13\end{array}$ & $\begin{array}{r}0 \\
0 \\
0 \\
0 \\
0 \\
0 \\
0 \\
130 \\
0 \\
0 \\
0 \\
0 \\
0\end{array}$ & $\begin{array}{r}42 \\
418 \\
2089 \\
13 \\
1,59 \\
253 \\
114 \\
683 \\
1,230 \\
1,104 \\
667 \\
143\end{array}$ \\
\hline
\end{tabular}

Sect foolnowes al ead of table 
Table 24. U.S. Flectric Utllity DSM Program Indireet Utitity Costs by North American Electric Reliability Concil Region and Hawati by Cost Categary, 1996 (Thousand Dollars) (Conlinued)

\begin{tabular}{|c|c|c|c|c|c|}
\hline 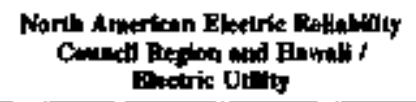 & Ambialutroline & Merketims & $\begin{array}{l}\text { Moonlawing } \\
\text { and } \\
\text { Evaluetion }\end{array}$ & OAner I & Twal Inairex \\
\hline
\end{tabular}

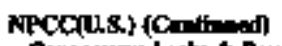

Comecturin Ligh \& Powns Co

Conoectucit Vulley bec Co It:

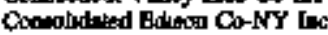

Easten Edtot Do

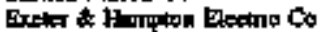

Fruchlourg oba bec Lght Co

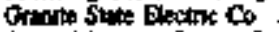

Gran Movntaro Power Cors

Hinphar Chy of

Balyowe Cir of

Janetoin City of

Long blond Leghens Co

Maves Publuc Service Co

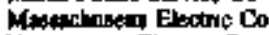

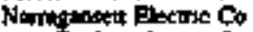

Now Eugladd thowt Co

Nor Hanpulare Bloc Coop Ine

Newpont Eledtus Cimp

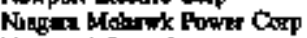

Nantood Cty of

Oranat Rockland Jula lac

Poner Aodranty of Stere of NY

Puble Serves Co of NH

Rochenter Gas \& Elome Com

Shewtbory Town of

Unead thrmunimg Co

Vermont Eletec Coop lne

Wentem Mapsatumacill Elec Co

Nitce(US.) Total

SERc

Auken Eloctne Coop Ioc

Alsbemi Fectinc Coop loc

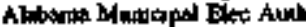

Arbanam Pourt to

Albern- City of

Berticley Blectuc Copp toc

Black Fuwer Eloconc Cocp loc

Brunumet Blactinc Member Conp

Condm Chy of

Carrol Eective Merber Cop

Contril Coorgy I Menber Cop

Cewal Vurinta Blectic Doop

Choctuwh the Blec Copp tec

Cobt Elextre Miembarshp Corp

Convess-Fayste El Mombar Cor

Dowdet Gity of

Sosky Corabued Ualury Syater

Eist Pount Cty of

Elmabeth Cly Cury of

Fondietd Eloctinc Coop Inc

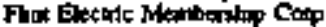

Fonth Kes El Coop Asu be

Flonda Powe a Ljofi Co

Fionda Powes Corp

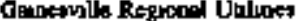

Greamila Uthines Corto

Cheysune porter Conte

Hojwood Binawe Manber Cosp

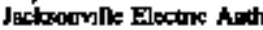

Jeffason Festinc Mromber Cop

Jowen-Ondow Fine Mlonber Corp

Lexland Chy of

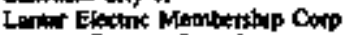

Lranen Eectric Coop hos

loonabur $\mathrm{Cxy}$ of

Ser fortinotins of and of chipls

\begin{tabular}{|c|c|c|c|c|}
\hline 1,837 & $\theta$ & 1,514 & 929 & 4,280 \\
\hline 41 & 0 & & 0 & 4 \\
\hline 3.145 & 0 & 3,600 & 0 & 6,205 \\
\hline 634 & 160 & I2] & 0 & 915 \\
\hline 135 & 1 & 14 & 0 & 150 \\
\hline 152 & 0 & 18 & 0 & 170 \\
\hline 146 & 43 & 4] & t & 230 \\
\hline 344 & 0 & 65 & 391 & $\$ 0$ \\
\hline 0 & 4 & 0 & 0 & 4 \\
\hline 30 & D & 0 & D & 30 \\
\hline os & 0 & 0 & 0 & 95 \\
\hline 1,045 & 0 & 9d3 & 623 & 2,611 \\
\hline 13 & I & 0 & 38 & 52 \\
\hline 3,534 & 1,122 & 1.607 & 0 & 6,283 \\
\hline $12 n$ & 194 & 468 & 0 & 1,884 \\
\hline 219 & 0 & 0 & 0 & 119 \\
\hline 352 & 262 & 45 & 0 & 659 \\
\hline 104 & 27 & 77 & to & 158 \\
\hline 500 & 0 & 24 & 0 & 524 \\
\hline 0 & 5 & I2 & 0 & 3] \\
\hline 166 & 204 & 275 & 189 & 54 \\
\hline 1,942 & 0 & 0 & 0 & 1,942 \\
\hline 87 & 0 & 0 & 109 & 196 \\
\hline 200 & 0 & 118 & 0 & 318 \\
\hline 0 & 0 & 0 & 5 & 5 \\
\hline 252 & 19 & $30 ?$ & 0 & 57 \\
\hline 164 & 0 & 0 & 0 & 164 \\
\hline 21714 & 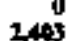 & 1173 & $\begin{array}{r}497 \\
2901\end{array}$ & $\begin{array}{r}1,972 \\
36,70\end{array}$ \\
\hline
\end{tabular}

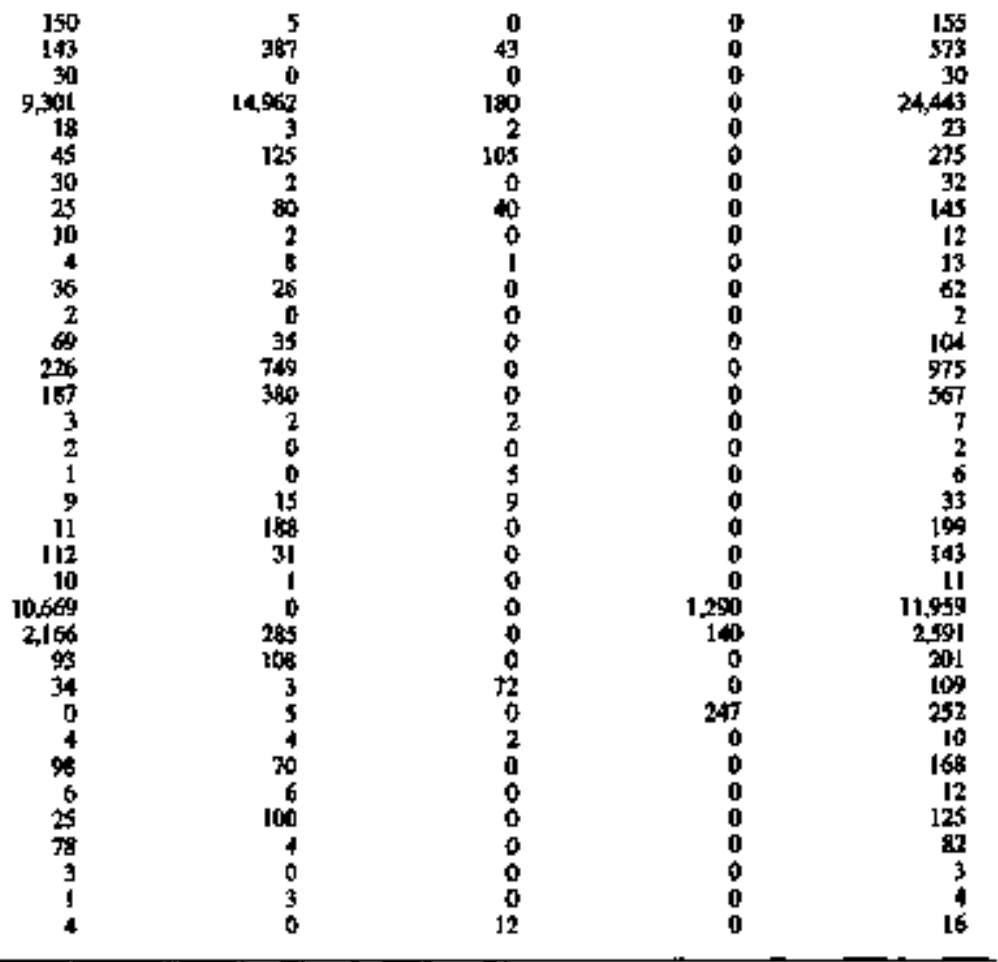


Table 24. U.S. Electric Utility DSM Program Indirect Uti:ty Costs by North American Electric Reliability Conncil Region and Hawal by Cost Colegory, 1996 (Thousand Doilars) (Continued)

\begin{tabular}{|c|c|c|c|c|c|}
\hline 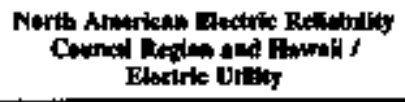 & 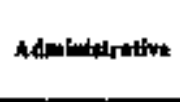 & Mestomed & 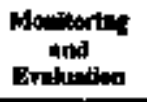 & Other 1 & 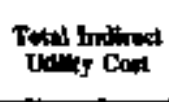 \\
\hline
\end{tabular}

BRRC [Cwotinead)

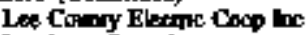

lecoumen Ory of

Lumbion Gity of

Mapaspess Ary of

Mecldaiburs Elecone Cocp lne

Mrdoutun Eketric Coop loc

Now Bern City of

Now Ruwer Lith a Power Co

North Corditi Edut M P A

Nonh Carolins Mun Power Agor

Norther Nect Eex Coop Inc

Norther Virtellat Elec Coop

Orapobers City of

Orlando bihties Coms

Pulnetro Blocenc Orop inc

Pinde Cocro Eestic Coop

Rayle Electis Klomberimp Corp

Ready Crat lmorowament Dut

Soulla Rutd Ext Montion Con

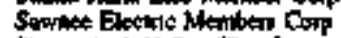

Stbermdoal Valley Elec Coop

Stajng Rurar Blec Power Asu

Snuthileld Toun of

Souh Coroline Pub Sery Awh

Southonde Bectuc Coop I Ix

Surter Elactine Coop ins

Tothlateses Cty of

Tamps Elocrix o

Tr-County Bec Netmber Corp

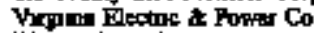

Wition Cry of

York Eloctric Coop be

S:AIC TE:

SPP

Alou Cry of

Cyun Elostric Poner Cowp bye

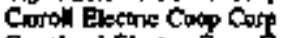

Cragluead Electne Coop Cop

Dencin Orty of

Eirs Elene Coop Com

Golder Spiend Eloc Coop lac

Grundy Bextinc Coop Inc

Independetise Ory of

Kunts Cuy City of

Konga Cty Power a logt Co

Kagran Electinc Prower Coop line

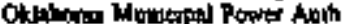

Orat Electric Coop lne

Ped Jen Elestne Bow Conp

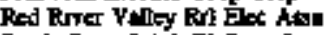

Soulh Ceobal Ait El Coop inc

Southmesuan Pobles Servoce $C_{0}$

Vephens Vellay Des Coop lnc

Whate Ruth Vaifoy El Cosp Iac

woodhuff Electre Cosp Comp

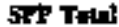

WSCC(U.3)

Alareda Crty of

Aluean Coty of

Arteron Puble Service to

Bomevil Pons Aind

Bonlder Bty Giry of

Bontuful Cily C.y of

See fooknows at tod of nollo

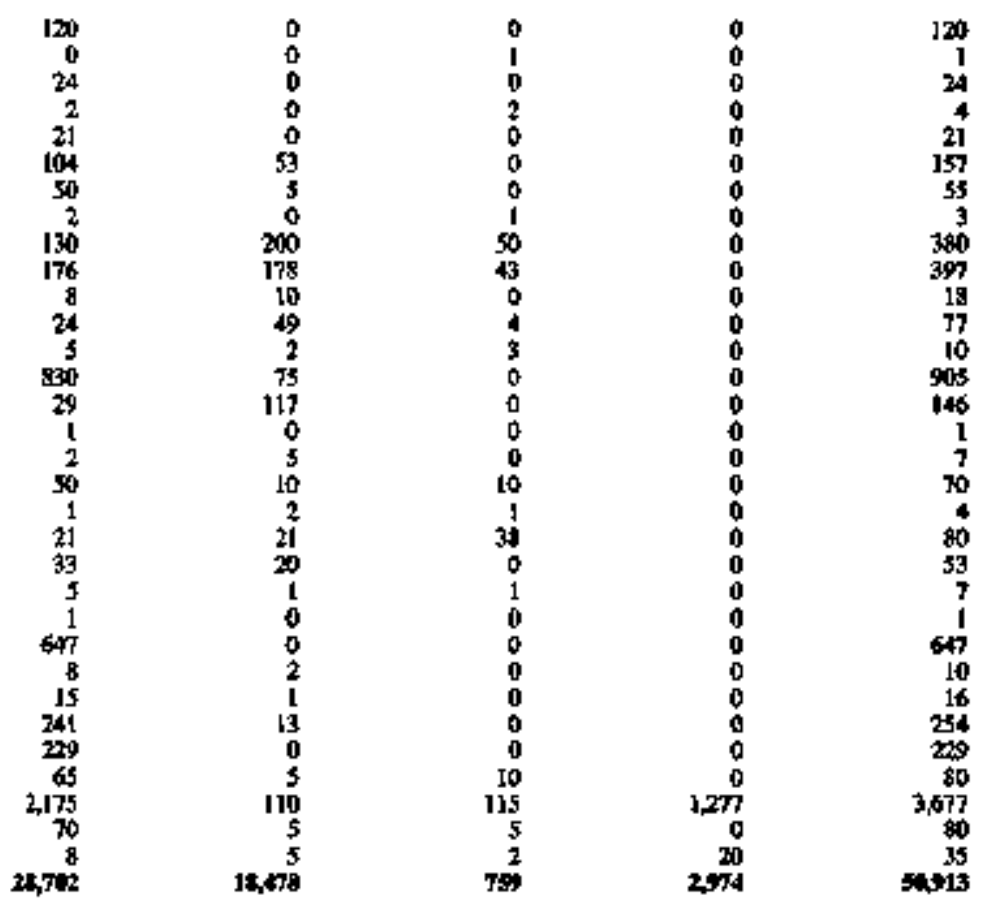

\begin{tabular}{|c|c|c|c|c|}
\hline $\begin{array}{r}1 \\
110 \\
5 \\
37 \\
40 \\
5 \\
5 \\
70 \\
25 \\
249 \\
0 \\
2 \\
17 \\
1 \\
5 \\
2 \\
0 \\
446 \\
5 \\
0 \\
0 \\
045\end{array}$ & $\begin{array}{r}0 \\
45 \\
0 \\
35 \\
20 \\
5 \\
0 \\
20 \\
2 \\
142 \\
0 \\
22 \\
15 \\
0 \\
0 \\
0 \\
0 \\
56 \\
0 \\
0 \\
0 \\
12\end{array}$ & $\begin{array}{r}1 \\
0 \\
10 \\
29 \\
0 \\
5 \\
0 \\
5 \\
5 \\
0 \\
0 \\
15 \\
0 \\
0 \\
15 \\
2 \\
0 \\
108 \\
12 \\
6 \\
60 \\
20\end{array}$ & $\begin{array}{r}0 \\
0 \\
0 \\
0 \\
0 \\
0 \\
55 \\
0 \\
0 \\
0 \\
150 \\
0 \\
0 \\
0 \\
32 \\
0 \\
1 \\
0 \\
0 \\
0 \\
0 \\
206\end{array}$ & $\begin{array}{r}605 \\
15 \\
101 \\
60 \\
15 \\
60 \\
95 \\
32 \\
391 \\
150 \\
39 \\
32 \\
1 \\
52 \\
1 \\
1610 \\
17 \\
6 \\
10 \\
2,94\end{array}$ \\
\hline
\end{tabular}

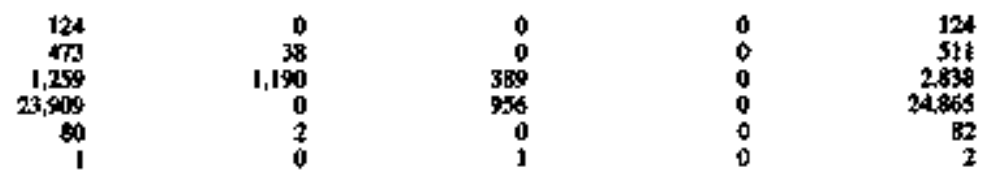


Tabje 24. US. Elextric Uttility DSM Program Indirect Uility Costs by North American Electric Reliability Counci Rezion and Hawali by Cost Category, 1996 (Thousand Dollars) (Continued)

\begin{tabular}{|c|c|c|c|c|c|}
\hline 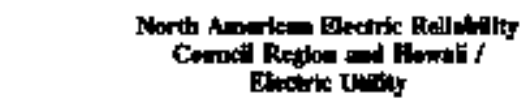 & Adnotolitative & Manketing & $\begin{array}{l}\text { Moithoriat } \\
\text { and } \\
\text { Bratuation }\end{array}$ & 0 & $\begin{array}{c}\text { Tatol Inilnat } \\
\text { Utilk' Cond }\end{array}$ \\
\hline 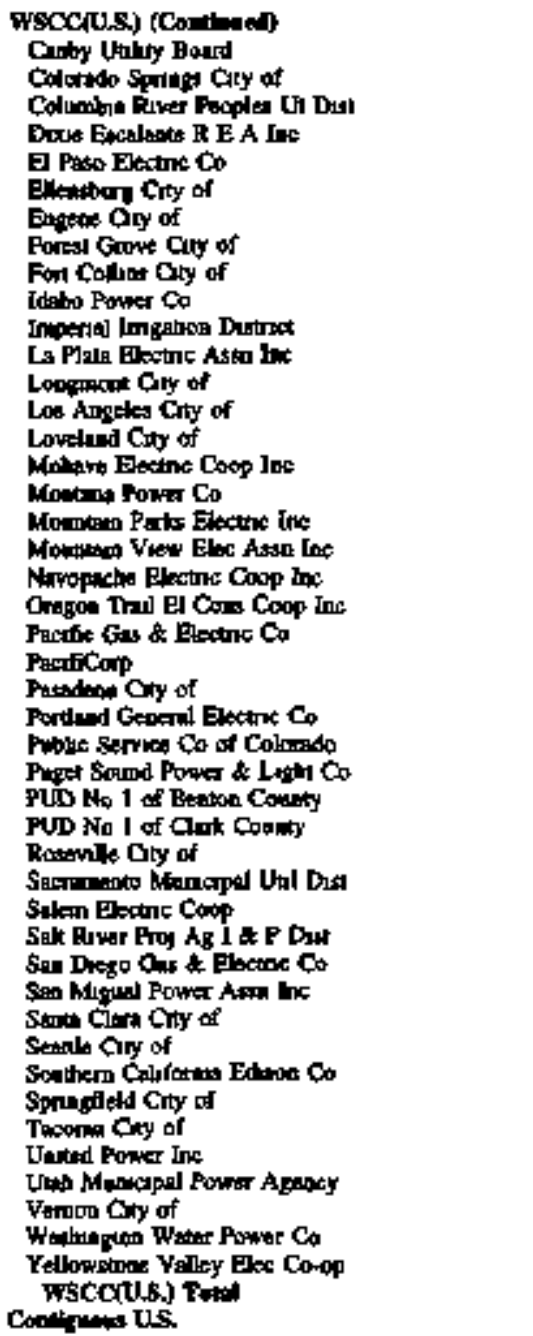 & 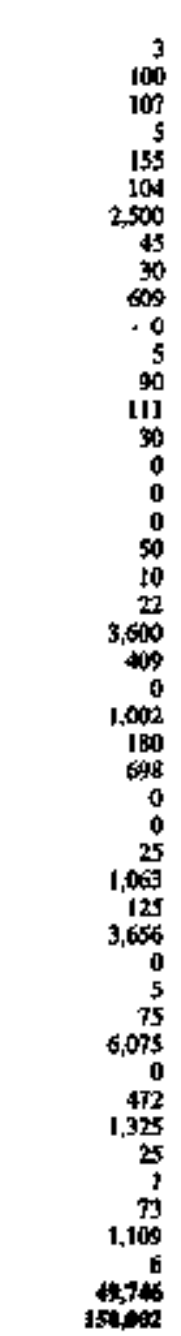 & $\begin{array}{r}0 \\
0 \\
30 \\
0 \\
0 \\
0 \\
30 \\
0 \\
0 \\
0 \\
41 \\
2 \\
0 \\
165 \\
15 \\
5 \\
1,132 \\
20 \\
20 \\
4 \\
15 \\
0 \\
11 \\
0 \\
0 \\
0 \\
0 \\
25 \\
0 \\
3 \\
0 \\
15 \\
628 \\
0 \\
5 \\
0 \\
0 \\
0 \\
0 \\
0 \\
40 \\
1 \\
0 \\
0 \\
17 \\
3,494 \\
50,920\end{array}$ & 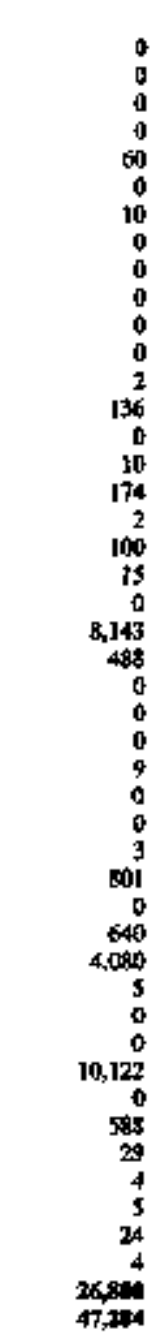 & 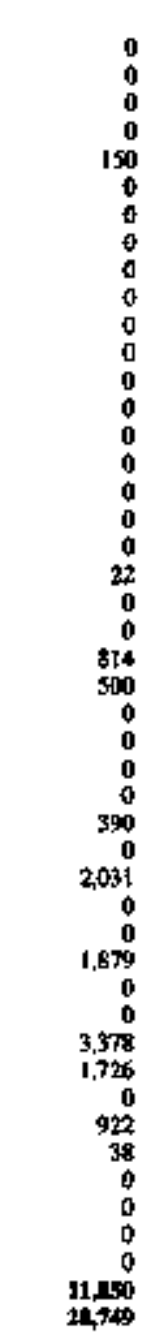 & 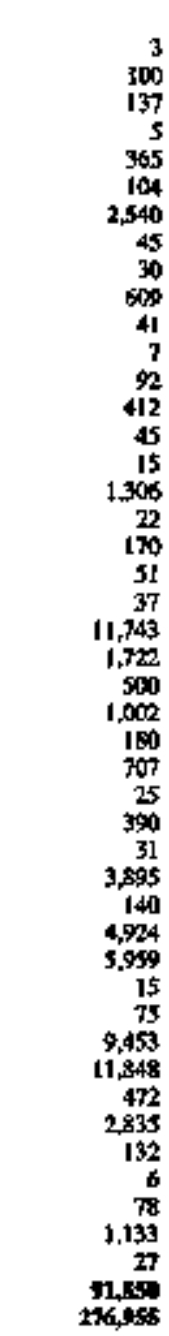 \\
\hline 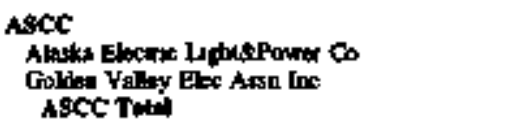 & $\begin{array}{l}5 \\
91 \\
94\end{array}$ & $\begin{array}{l}2 \\
12 \\
\mathbf{1}\end{array}$ & $\begin{array}{l}2 \\
0 \\
2\end{array}$ & 0 & $\begin{array}{r}9 \\
103 \\
112\end{array}$ \\
\hline 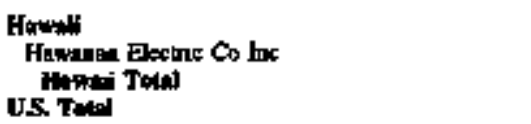 & $\begin{array}{r}799 \\
75 \\
19404\end{array}$ & $\begin{array}{r}30 \% \\
31,241\end{array}$ & $\begin{array}{r}215 \\
215 \\
47,501\end{array}$ & $\begin{array}{r}231 \\
231 \\
2 \times 10\end{array}$ & $\begin{array}{l}1,542 \\
1,50 \\
\operatorname{mit}_{4}, 99\end{array}$ \\
\hline
\end{tabular}

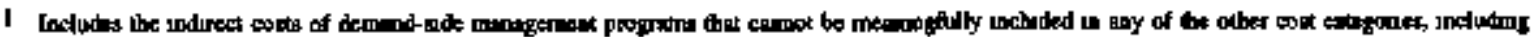

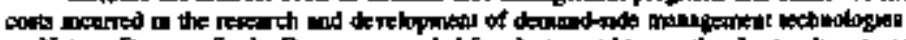

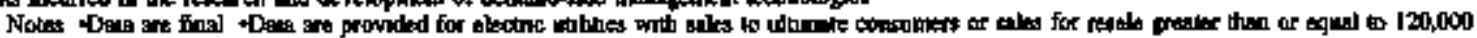
ncejpivithour

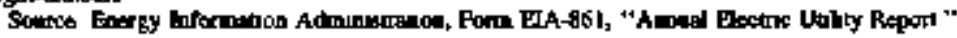



Appendix A

Technlcal Notes 



\section{Technical Notes}

\section{Source of Data}

The U.S. Electric Villity Demand-Side Management report is prepared by the Coal and Electric Data and Renewables Division; Office of Coal, Nuclear, Electric and Alternate Foels: Energy Information Administration (EIA); U.S. Department of Energy (DOE). Data published in the U.S. Electric Utility Demand. Stde Management report are compiled from the Form EIA-861, "Anoul Electric Utility Report," which is summarized below:

\section{Form EIA-86T}

The Form ELA-861 is a mandatory census of electric utilities in the United States, its territories, and Puerto Rico. The Forra EIA-861 data contained in this publication are for the United States only. The survey is used to collect information on power production and sales of electricity from approximalely 3,200 electric utilities. The data collected are used to update the clectric utility framo database maintained by the EIA. This database supports queries from the Executive Branch, Congress, other public agencies, and the general public. Summary data from the Form EtA-861 are also contained in the Electric Power Annsal Volune II; Electric Sales and Revenue; Financial Sta. tistics of Major U.S. Investor. Owned Electric Utilities; Financial Statistics of Major U.S. Publichy Owned Electric Utilities; Annual Entergy Outlook; Electric Trade in the United States, Annwal Energy Review, Monthly Energy Review, and Electric Power Monthly. These reports present aggregate totals for electric uti]ities on nationsl, State, and NERC Region levels and by ownership class and consumer class of service.

Defnand-side management (DSM) data are collected on Schedule V, "Demand-Side Management Informstion," of Form EIA-861. Collected are date on DSM costs, annual and incremental offects for ouergy savings and for actural and potential peak load roductions. Also collected is infotmation on the end use and type of energy efficiency programs. DSM dats collected on Form ELA-861 are estimated by electric utilities based on engineering data, statistical analysis, or other estimation methods.
EJA cellects information on DSM activities from all tutilities with DSM programs. DSM data are aggregated at the NERC region and consumer sector levels. Utilities with sales to ultimate consumers or sales for resale greater than of equal to 120,000 megawatthours report incremental peak load teductions and energy effects for the reporting year, annual pesk load reductions and energy effects for the reporting year and first- and fiftb-forecast years, itemized direct and indirect utility costs and nonutility cost attributable to DSM programs for all 3 years, end use and type of eotrgy effíclency programs. Annual and incremental effocts for the reporting year are reported by consumer sector (residential, commercial, industrial, other) for each program category (energy efficiency, direct load control, isterruptible lood, other load management, other DSM programs, and load building). Forecast peak load reductions and energy effects are reported by program categary with all consumer sectors combined. Utititios with saltes to ultimale consumers and sales for resale less than 120,000 megawatthours report selected items: incremental peak load reductions and energy effects, total utility cost, total monutility cost, and total DSM cost for the reporting year and first- and fffth-forecast yeats, end use and type of energy efficiency programs. In years prior to 1992, utilities with sales for resale and sales to. oltimate consumers less than 120,000 megawatthours did not report on DSM activities.

Instrument and Design History. The Form EIA-B61 was implemented in January 1985 to collect data as of year-end 1984. Schedule V, "Denand-Side Manggement Information," was added to the survey in 1990 to collect data for year-ead 1989. Schedule V was revised for the 1991 collection and again for the 1993 year-end collection. The Federal Energy Administration Act of 1974 (Public Law 93-275) and the Energy Policy Act of 1992 (Public Law 102-4B6) define the legislative autbority to collect those data.

Data Procgssing. The Form EIA-g61 is mailed to the respondents in January to colleet data as of the end of the preceding calendar year. The completed forms are to be returned to the EIA by April 30 . Internal edit checks are performed to verify that current data are comparable to data reported the previous year. Respondents are telephoned to obtain clarification of reported data and to obtain missing data. 


\section{Voltege Peduction}

Voltage reduction, though nol considered a DSM program, may be used by utilities to reduce load since power provided to the consumers is a function of both voltage and curtent. Voltage reduction is mainly used in emergency situntions, althongh some uljlities use it to reduce demand during peak load periods under nortsal operating conditions.

During normal operating conditions, utilities provide service to retait consumers within a range of voltages (e.8., $120 \mathrm{w}+5$ percent). States gederally promulgate rules that describe the service utilities must provide to customers, including voltage levels. Diring emer* gency situations, utilities are allowed to go beyond the normal operating range to a limited extent. Most systems that use voltage reduction during emergencies limit the variation to a maximum of 5 percent ontside of normat operating limits, but some $\mathrm{go}$ as high as $\mathrm{g}$ percent. The reduction applied may be any level ap to the maximum, depending on the circumstances. Although the emergency voltage reductions go catside of the normal ranges, they are implemented for short periods of time (as littie as 10 minutes 10 an hour). Voltage reduction is effected by reducing the voltage at customer-level substations (distribution system), either manually or remotely, if the utility system is fully automated. A voltage reduction can be made for one area of a utility's service territory, or for an entire utility system.

The amount of power that is saved when roltage is reduced depends on many factors including the types of load and the relative proportions of thase loads at the time the voltage is redoced. Since load mix and level varies by season and time of day, the impacts of voltage reduction will vary aceordingly. The potential peak load savings thet may be achieved under a sel of specific circumstances for a 5 percent reduction in voltage, can range from negligible to 5 percent of summer peak load, with most savings being less than 3 percent of winter or summer peak lond.

Some utitities also use the term "voltage reduction" to include improvements in their distribution system that allow them to operate at lower nominal roltages. By investing in improved voltage regulators, line reconductoriag, and other distribution equipment, utilities can lower substation operating volioge and still provide customers with adequate voltage, thereby saving energy. When the savings arte adequate to justify the iovestment, utilities may implement such a program and refer to it as voltage reduction or conservation voltage reduction. 


\section{Quality of Data}

The Office of Coal, Nuclear, Electric and Alternate Fuels (CNEAF) is responsible for routine data improvement and quality assurance activities. All aperations in this office ate done in accordance with formal standards ostablished by the EIA. Data improvement efforts include verification of datakeyed input by automatic computerized methods, editing by subject matter specialists, and follow up on nonrespondents. The CNEAF office supports the quality assurance efforts of the data collectors by providing advisory reviews of the structure of information requirements and of proposed designs for new and revised data collection forms and systems. Once implemented, the actual performance of worting data collection systems is also validated. Computerized respondent data files are checked to identify those who fail to respond to the survey. By law, nonrespondents may be fined or otherwise penalized for not filing a mandatory EIA dath form. Before involing the law, the EIA tries to obtain the required information by encouraging cooperation of nonrespondents.

Completed forms received by the CNEAF office are sorted, screened for completeness of reported information, and keyed onto computer tapos for siorage and transfer to random access databases for computer processing. The informatiou coded on the computer tapes is mannally spot-checked against the forms to certify accuracy of the tapes. To ensure the quality standards established by the EIA, formulas that use the past history of data values in the datobase bave been designed and implemented to check dala input for ortors sutomatically. Date values that fall outside the ranges prescribed in the formulas are verified by telephoniog respondents to resolve any discrepancies.

\section{Data Editing Systom}

Data from the surveys are edited using automated systems. The edits inciude both deterministic checks, in which records are checked for the presence of required fields and their valjdity; and statistical checks, in which estimation techniques are used to validate data according to their behavior in the past and in comparison to other current ficlds.

\section{Confidentiallty of the Data}

The data collected on the Form EIA-861 used for input to this report are not confidential.

\section{Rounding Rules for Data}

Given a number with $r$ digits to the left of the decimal and $\mathrm{d}+\mathrm{t}$ digits in the fraction part, with $\mathrm{d}$ being the place to which the number is to be rounded and $t$ being the remajning digits which will be truncated, this number is rounded to $\mathrm{r}+\mathrm{d}$ digits by adding 5 to the $(r+d+1)$ th digit when the number is pasitive or by solvtracting 3 when the number is aegative. The $t$ digits are then truncated at the $(r+d+1)$ th digit. The symbol for a rouaded aunber truncated to zeto is (*).

\section{Percent Difference Calculation}

The following formula is used to calculate percent differences.

Percent Difference $=\left(\frac{x\left(t_{2}\right)-x\left(t_{1}\right)}{x\left(t_{1}\right)}\right) \times 100$,

where $x\left(f_{1}\right)$ and $x\left(b_{2}\right)$ denote the quantity at year $t_{1}$ and subsequent year $t_{2}$

\section{CNEAF Dota Rovision and Polloy}

The Office of Coal. Nuclear, Electric and Alterntate Fuels bas adopted the following policy with respect to the revision and correction of recurreat data in energy publications:

1. Annual survey data collected by this office are published either as preliminary or final when first appearing in a data report. Data initially released as preliminary will be so noted in the report. These data will be revised, if necessary, and declared final in the next publication of the data.

2. The magnitude of changes due to revisions experienced in the past will bo included in the data reports, so that the reader can assess the accuracy of the data.

3. After data are published as final, cotractions will be made only in the event of a greater than one percent difference at the national level. Corrections for differences that are less than the before-mentioned threshold are left to the discretion of the Office Director.

The U.S. Electric Utility Demand-Side Management (DSM) report presents the most current annual data arailable to the EIA. The statistics may differ frod those poblished previously in ElA pablications due to corrections, revirjous, or other adjustments to the data subsequent to its original release. The status (preliminary versus final) of DSM data published by EIA follows:

- U.S. Elextrte Utility Demand-Side Manogement

Data on demand-side managemed from the Form EIA-86I are fípat.

- Electric Power Amnunl Vohrne II 1996

The chapter in the Electric Power Ankual Volume II for DSM contains dats on demand-side management from the Form ElA-861. Data for 1996 and previous years are final. 


\section{Use of the Glostary}

The terms in the glossary have been defined for general use. Restrictions on the definitions as used in these data collection systems are included in each definition when necessary to define the terams as they are used in this report.

\section{Acronyms and Abbrevlations}

CNEAF - Office of Coal, Nuclear, Electric and Alternate Fuels

DOE - Department of Energy

DSM - Demand-Side Management

EIA - Energy Information Administration

EPACT - Energy Policy Acl of 1992

GWh - Gigawatthour

HVAC - Heating, Ventilation, and Air Conditjoning

IRP - Integrated Resource Planning
kW - Kilowatt

kWh - Kilowatthour

MW - Megawatt

MWh - Megawatthour

NERC - North American Electric Relisbility Council

The NERC regions are:

ASCC - Alaskan System Coardination Council

ECAR - East Central Area Reliability

Coordination Agreement

ERCOT - Electric Reliability Council of Texas MAIN - Mid-America Intereonnected Network

MAAC - Mid-Atlantic Area Council

MAPP - Mid-Continent Area Power Pool

NPCC - Northeast Power Coordinating Council

SERC - Southeastern Electric Reliability Council SPP - Soutbwest Power Pool

WscC - Western Systems Coordinating Counci]

NTIS - National Technical Information Service

TOU - Time-of-Use 
Flgure A1. North American Pollabilly Counell Reglont for the Contiguous United Stutes and Alwoka

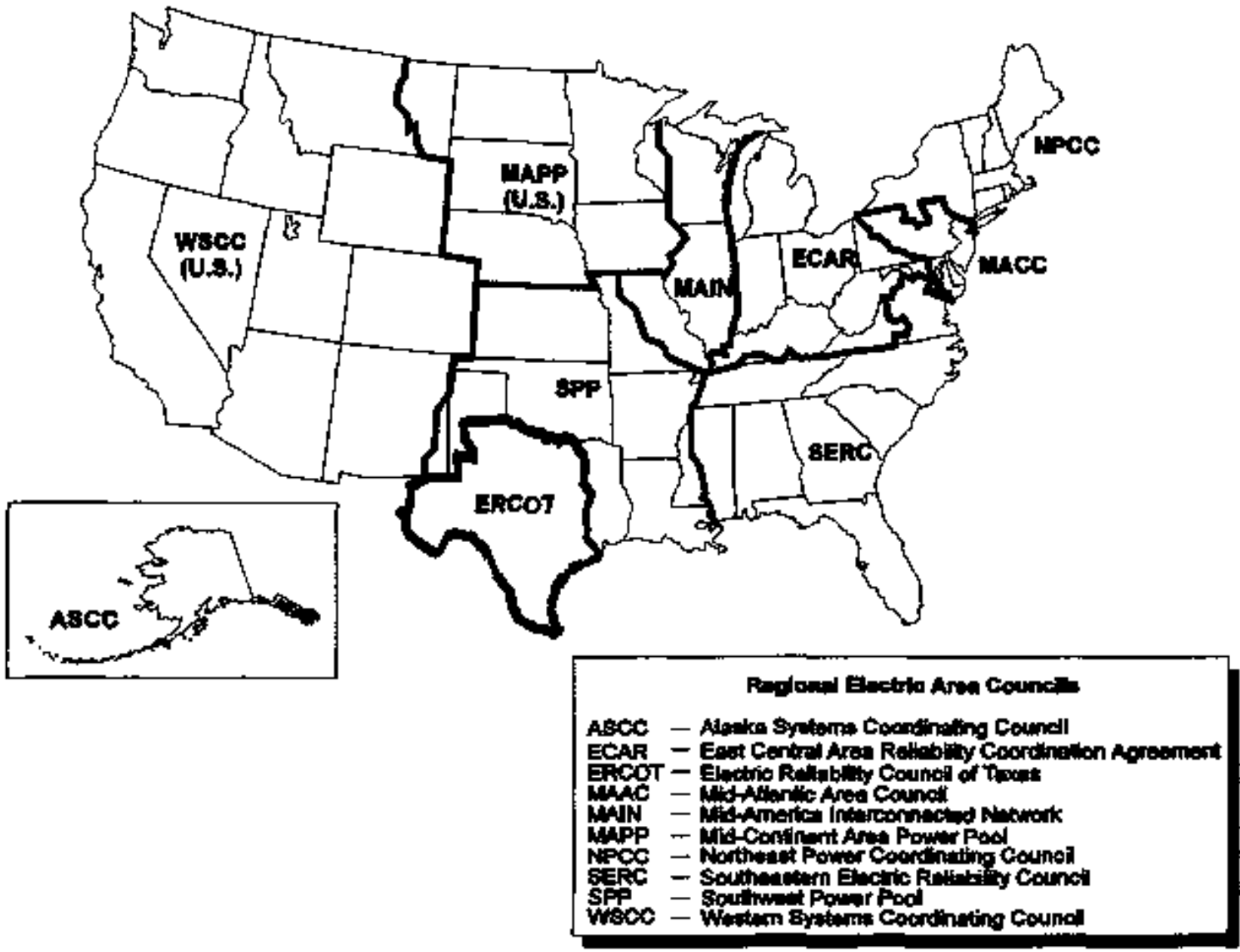

Sounce: North American Electric Reliabrity Council. 


\section{Obtalning Coples of Data}

The data are available on machine-readable tapes. Tapes may be purchased by using Visa, MasterCard. or American Express cords as well es money orders or checks payable to the National Technical Information Service (NTIS). Purchasers may also use NTIS and Goveroment Printigg Office depositary accounts. To place an order, contact:

National Technical Information Service (NTIS)

Office of Data Base Services

U.S. Department of Commerce

5285 Port Royal Road

Springfield, Virginia 22161

(703) $487-4650$

The data for 1992, 1993, 1994, 1995, and 1996 filed on the Form ELA-861 are also available on the
Internet in compressed format through FTP at ftp.eia.doe.gov, or through use of a wotld-wido-web browser such as Netscape at wow.eia.doe.gov, in the jpub/energy subdirectory.

The database may also be purchased on personal compoter diskettes ( $31 / 2$ or $51 / 4$ ) using Mastercard or Visa as well as money order or check payable to the U.S. Department of Energy. To place an order, contact:

Office of Scientific and Techtitical Information U.S. Depatment of Energy

Request Services

P.O. Box 62

Oak Ridge, Tennessee 37831

(615) 576-8401 or Fax (615) 576-2865

Table AL. Unithof Mensure Bquitwants

\begin{tabular}{|c|c|}
\hline Intis & Bentitudunt \\
\hline 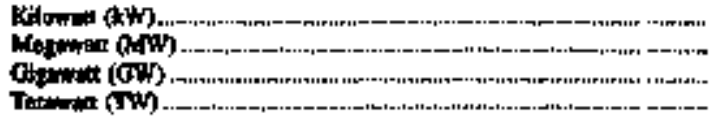 & 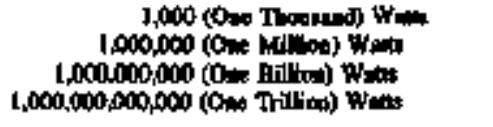 \\
\hline 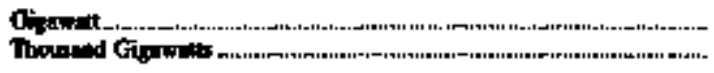 & 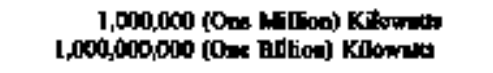 \\
\hline 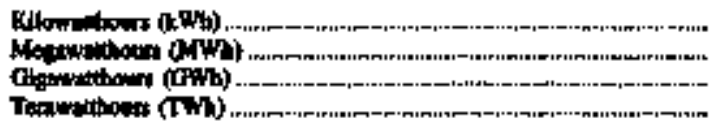 & 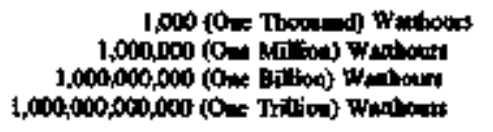 \\
\hline 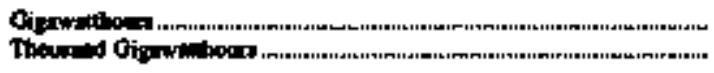 & 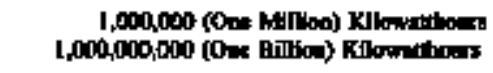 \\
\hline
\end{tabular}

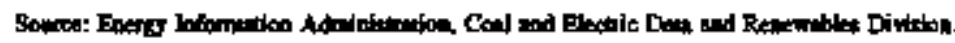


Appendix B

Glossary 



\section{Appendix B}

\section{Glossary}

Actual Peal Load Reductions: The actual reduction in annual peak load (measured in kilowatts) achieved by consumers that participate in a utilily DSM program. It reflects the real changes in the demand for electricity resulting from a utility DSM program that is in effect at the sarne time the utility experiences its annul pexk load, as opposed to the installed peak load reduction capability (i.e., Potential Peal Load Reduction). It should account for the regalat cycling of energy efficient units during the period of annual peak load.

Annual Effects: The total effects in energy use (measured in megawatthours) and peak load (meas. ured in kilowat(s) caused by all participants in the DSM progrions that are in effect diring a given year. It includes new and existing participants in existing programs (those inplemented in prior years that are in place during the given year) and all participants in new programs (those implemented during the given year). The effects of new participants in existing programs and all participants in new programs should be based on their start-up dates (i.e., if participants enter a program in July, only the effects from July to December should be reported). If start-up dates are unknows and cannot be reasonably estimated, the effects can be annualized (i.e., assume the participants were initiated into the program on January 1 of the given year). The Annual Effects should consider the usteful life of efficiency measures, by accoupting for building demolition, equipment degradation and attrition.

Appllances: Energy Efficiency program promotion of high efficiency appliances such as dishwasbers, ranges, refrigerators, and freozers in the residential, commercial, and industrial sectors. Includes programs aimed at improving the efficiency of refrigeration equipment and electrical cooking equjpment, including replacement. It also includes the promotion asd identification of bigh efficiency appliances in retail stores using a labeling system different from the Federally-mandated Energy Guide. Energy Efficiency program promotion of high tfficiency cooling and heating appliances are included under Cooling System and Heating Systent, respectively.

Asset: An ecopomic resobrce, tangible or intangible, which is expected to provide benefits to business.

A rerage Revenue per Kdlowntthonr: The average revenue per kilowatthour of electricity sold by sector (residential, commercial, industrial, or other) and geographic ares (State, Census division, and National), is calculated by dividing the tolal monthly revenue by the corresponding total monthly sales for each sector and geographic area.

Census Dlvisions: The nine geographic divisions of the United States established by the Bureat of the Census, U.S. Department of Commerce, for the purpose of statistical analysis. The boundaries of Census divisions coincide with State boundaries. The Pacific Division is subdivided into the Pacific Contiguous and Pacific Noncontiguous areas.

Cogenerator: A generating facility that produces electricity and another form of useful thermal energy (such as heat or steam), used for industrial, commetcial, beating, or cooling purposes. To recejve status as a qualifying facility (QF) under the Public Utiljty Regulatory Policies Act (PURPA), the facility murst produce electric energy and "abother form of useful thermal energy through the sequential use of energy," and meet certain ownership, operating, and efficiency criteria established by the Federal Energy Regulatory Commission (FERC). (See the cote of Federal Regulations, Title 18, Part 292.)

Coincidental Peak Laad: The sum of two or more peak loads that occur in the same time interval.

Commerctal: The commercial sector is generally defined as nonmanufacturing business establishments, including hotels, motels, restansants, wholesale businesses, retail stores, and health, social, and educational institutions. The utility may classify commercial service as all consumers whose demand or annual use exceeds some specified limit. The linuit may be set by the utility based on the sate schedute of the utility.

Commercial Opertion: Commercial operation begins when control of the loading of the generator is turned over to the system dispatcher.

Cooling System: Energy Efficiency program promotion aimed at improving the efficiency of the cooling delivery system, including replatectent, in the residential, commercial, or industrial sectors.

Cooperative Electric Utility: An electric utility legally established to be owned by and operated for the benefjt of those using its service. The utility company will generate, transmit, and/or distribute supplies of electric energy to a speciffed area not 
being serviced by another utility. Strch ventures are generally exempt from Federal income thx laws. Most electric cooperatives havo been intially financed by the Rural Electrification Administration, U.S. Department of Agriculture.

Demand (Electric): The rate at which electric energy is delivered to or by a system, part of a system, or piece of equipment, at a given instant or averaged over any designated period of time.

Denand-Side Manageneat: The planning, implementation, and monitoring of utiljty activities desigued to encourage consumers to modify patierns of electrifetty usage, including the timing and level of electricity demand. It refers only to energy and loadshape modifying activities that ase undertaken in respouse to utility-administered programs. It does not refer to energy and lowd-sbape changes arjsing from the normal operation of the marketplace or from govermment-mandated energy-affjciency standards. Demand-Side Management (DSM) covers the complete range of load-shape objectives, inisluding strategic coaservation and load managentenl, as well as stralegic load growth.

Demmod-Stde Mans gement Cout. The cost incurred by the utility to achieve the capscity and etaergy savings from the Demand-Side Managemtent Program. Costs (expenditures) incuted by consunters or third parties are to be excluded. The costs are to be reported is nominal dollars is the year in which they are inculred, regardless of when the savings occur. Program costs include expensed items incurred to implement the program, incentive payments provided to consumers to justait Demand-Side "Vanagement measures, and annual operation and maintenance expenses incurred during the year. Utility costs that are general, administrative, or not specific to a particular Demand-Side Management category are to be included in "other" costs.

Direct Load Control: Refers to program activities that can interrupe consamer load at the tine of annual peak load by direct control of the utility system operator by interrupting power supply to individuat appliances or equipment on consumer premises. This type of control usually invoives residential consumers. Direct Load Control excludes Interruptible Load and Other Load Management effects. (Direct Load Control, as defined here, is synonymous with Direct Loaf Control Management reported to the North American Electric Reliatsitity Council on. the roluntary Office of Energy Emergency Operstions Form OE-411, "Coorojinated Regional Bulk Powet Supply Program Report," with the exception that anoual peak load effects are reported here and seusonsl (i.t.+ summer and winter) peak load effects are reported on the DE-411.)

Direct Uttlity Cost: A utility cost that is identified with one of the DSM program categories (i.e., Energy Efficiency, Direct Load Control, Intermujtible Load, Otber Load Managertent, Other DSM Programs, Load Building).
Electric Piant (Physical): A facility continining prime movers, electric generators, and auxiliary equjpment for converting mechanical, chemical, and/or fission energy into electric energy.

Electric Rate Schedile; A statement of the electric rate and the terms and corditions goveming its application, including attendant contract terms and conditions that have been accepted by a regulatory body with appropriate oversight authority.

Rlectric Utfilty: A corporation, person, agency, authority, or other legal entity or instrumentality that owns and/or operates facilities within the United States, its territories, or Puerto Rico for the generation, transmission, distribution, or sale of electric energy primarily for use by the public and files forms listed in the Code of Federal Regulations, Thue 18, Part 141. Facilities that qualify as cogenorators or sanal power producers under the Poblic Utility Regulatory Policies Act (PURPA) are not considered electric btilities.

Eowrgy: The capacity for doing work as measured by the capability of doing work (potential energy) or the conversion of this capability to molion (kinetic thergy). Energy has several forms, some of which are easily convertibje and can be changed to another form useful for work. Most of the world's convertible energy comes from fossil fuels that are burned to produce hetat that is then used as a transfer medium to mechanical or other means in order to accomplish tasks. Electrical energy is usually measured in kilowathours, while heat energy is vaudly measured in Britisb thermal units.

Enerty Charge: That portion of the charge for elec. tric service based upon the electric energy ( $k$ Wh) con. sumed or billed.

Enery Deliveries: Energy generated by one electric utility system and delivered to another system through one or more transmission lines.

Energo Bffects: The changes in a $\$$ gregate electricity use (measured in megawatthours) for customers that participate in a uthlity DSM program. Eneroy Effects should represent changes at the consumer meter (i.e. cxclude transmission and distribution effecta) and reflect only activities that are undertaken specifically in response to utility-adminjstered programs, including those activities inplonented by third parties under contract to the utility. To the extent possible, Energy Effects should exclude non-program related effects such as changes in energy usage attributable to nopparticipants, government-mandated energyefficiency standatds that legislole improvements in building and appliance energy usage, changes in consumer behavior that result in preater energy use afler injtiation in a DSM program, the natural operations of the marketplace, and weather and business-cycle adjustments.

Eaergy Bitictency: Refers to programs that are aimed at reducing the enetgy used by specific end-use devices and systems, bypically without affectiog the services provided. These programs reduce overalt 
electricity consumption (reported in megawathours), often without explicit consideration for the timing of program-induced savings. Such savings are generally achieved by substituting technically more advanced equipment to prodace the same level of end-use services (e.g., lighting. heating, motor drive) with less electricity. Examples include high-efficiency appliances, efficient lighting programs, high-efficiency heating, ventilating and air conditioning (HVAC) systems or control modifications, efficient building design, advanced electric motor drives, and beat recovery systems.

Enerby Receipts: Energy generated by one electric utility system and received by another system througb one or more transmission lines.

Energy Source: The primary source that provjdes the power that is converted to electricity through chentical, mechagical, or other means. Energy sources include coal, petroleum and petroleum products, gas, waler, uranium, wind, soblight, geothermal, and other sources.

Expenditure: The incorence of a liability to obtain an assef or service.

Fueilty: An existing or planged location or site at which prime movers, electric generalors, and/or equipment for converting mechantes, chemical, and/or nuclear energy into electric energy are situated, or will be situated. A facility may contain mote then one generator of either the same or different prime mover type. For a cogenerator, the facility includes the industrial or commercial process.

Federal Enersy Rezulatory Commisston (FERC): A quasi-independent regulatory agency within the Department of Energy having jurisdiction over interstate electricity \$ales, wholesale electric tates, hydroelectric licensing, natutal gas pricing, oil pipelixe rates, and ges pipeline certification.

Federal Power Commission: The predecessor agency of the Federal Energy Regulatory Commission. The Federal Power Commission (FPC) was created by an Act of Congress under the Federal Water Power Aet on June 10, 1920. It was charged originaliy with regulating the electric power and natural gas industries. The FPC was abolished on September 20, 1977, when the Department of Entergy was created. The functions of the FPC were divided between the Department of Energy ated the Federal Energy Regulatory Commission.

FERC: The Federal Energy Regulatory Commission.

Firn Power: Power or power-producing capacity intended to be available at all times during the period covered by a guaranteed commitment to deliver, eves under adverse conditions.

Forced Outige: The shutdown of a generating unit, transmission line or othey facility, for emergency reasons or a condition in which the generating equipment is unsvailable for load due to onarticipated breakdown.
Generating Unft: Any combination of physicaily connected generator(s), resctor(s), bojler(s), conbustion turbine(s), or other priute mover(s) operated together to produce electric power.

Generation (Electricity): The process of producing electric energy by transforming other forms of energy* also, the nuount of electric energy produced, expressed in watthours (Wh).

Gross Generation: The total anount of electric energy produced by the generating units at a generating station of stations, measured at the generator terminals.

Net Generation: Gross generation less the electrit energy consumed at the generating station for station use.

Generator: A machine that converts mechanical energy into electrical energy.

Cenerator Nameplate Capacity: The full-load continuous rating of a generator, prime mover, or other electric power production equipment under specific conditions as designated by the manufacturer. Instal]ed genertator nameplate rating is usually indicated on a bameplate physically attached to the generator.

Grid: The Iayout of an electrical distribution system.

Gross Generation: The total amount of electric energy produced by a generating facility, as measured at the generator terminals.

Hetting System: Eatrgy Efficieacy progtam promotion aimed at improving the efficiency of the heating delivery system, including replacement, in the residential, conmercial, or industrial sectors.

Incremental Effects: The annual effects in energy use (measured in megawatthours) and pesk load (measured in kilowatts) caused by tew participants in existing DSM programs and alf participants in new DSM programs during a given year. Reported Incremental Effects should be annuglized to indicate the program effocts that would have occurred had these participants been ititialed into the program on January 1 of the given year. Incremental effects are not simply the Annual Effects of a gived year mikus the Annual Effects of the prior year, sibce theso net effects would fail to account for program attrition, degradation, demolition, and participant dropouts.

Indirtect Utility Cost: A utility cost that may not be meaningfally identified with any particular DSM program category. Indirect costs could be attributable to one of several accounting cost categories (i.t., Administrative. Marketing, Monitoring \& Evaluation, Utility-Earned Incentives, Other). Accounting costs that are tnown DSM program costs should not be reported under Indirecl Utility Cost, rather those costs should be reported as Direct Utility Costs ander the appropriate DSM program calegory. 
Endustrial: The industrial sector is generally defined as mapufacturing, construction, mining agriculture, fishing and forestry extablishments (Standard Industrial Classification (SIC) codes 01-39). The vility may classify industrial service using the SIC codes, or based os demand or annual usage excecding some specifited limit. The limit may be set by the utility based on the rate schedole of the utility.

Interroptible Land: Refers to prograrn activities that, in accordance with contractual arrangements, can interrupt consumet load at times of seasonst peak load by direct control of the atility system operator or by action of the consumer at the direct request of the system operator. It usually involves cominercial and indostrial consumers. In some instance!l the load reduction may be affected by direct action of the system operator (remote trippiag) after notice to the consumer in accordance with contractus provisions. For exampie, loads that can be interrupted to fulfil] planning or operation reserve requirements should be reported as Interruptibie Load. Intortuptible Load as defined here excludes Dirtect Load Control and Other Load Management (Interruptible Load, als reported here, is synonymous with Interruptiblt: Demand reported to the North American Electric Reljability Council on the voluntary Office of Energy Emergency Operations Form OE-411, "Coordinated Regional Bulk Power Supply Program Report," with the exception that annual peak load effects are reported on the Form EIA-861 and seasonal (i.e., summer and winter) peak load offects are reported on the OE-411).

Kllowatt (kW): One thousand watts.

Kilowatthour ( 1 Wh): One thousand wattbours.

Liability: An amount payable in dollars or by future services to be tendered.

Load Bnilding: Refers to programs that are aimed at increasing the usage of existing electric equipment or the addition of electric equipment. Examples inciude industrial technologies such as induction beating and melting, direct are furnaces and infrared drying; cooking for commercial establishments; and hoat pumps for residences. Load Building should inclade programs that promote electric fuel substitution. Load Building effects should be reported as a negative number, shown with a minus sign.

Marketing Cost: Expenses directly associated whth the preparation and implementation of the strategies designed to encourage participation in a DSM program. The category excludes general market aod toad research costs.

Monitoring \& Evaluation Cont: Expenditures associated with the planning, collection, and analysis of data used to assess program operation and effects. It includes the activities such as load metering customer surveys, new technology testing, and program evaluations that are intended to establish or imptove the ability to monitor and evaluate the impacts of DSM programs, collectively or individually.
Maximum Demond: The gteatesi of all demands of the load that has occurred within a specified period of time.

Megawatt (MW): One million watts.

Megawatthour (MWh): One million wathours.

Net Capability: The maximum load-carrying ability of the equipment, exclusive of station use, under specified conditions for a given time interval, indepondent of the characteristics of the load. (Capability is delermined by design characteristics, physical conditions, adequacy of prime mover, energy supply, and operating limitations such as cooling and circulating water supply and temperature, headwater and tailwater elevations, and electrical use.)

Net Generation: Gross generation minus plant use from all electric utility owned plants. The energy required for pumping at a pumped-storage ptant is regarded as plant use and must be deducted from the gross generation.

Net Summer Capability: The steady hourly output. which generating equipment is expected to supply to system toad exclusive of auxiliary power, as demonstrated by tests at the time of summer peak load.

Net Winter Capability: The steady hourty outpue which generating equipment is expected to supply to system load exclusive of auxiliary power, as demonstrated by tests at the time of winter poak load.

New Construction: Energy-efficiency prograr promotion to encourage the building of new homes. buildings, atd plants to exceed standard governmentmandated energy efficiency codes; it may include major renovations of existing facilities.

Noncoincidental Penk Load: The sum of two or more peak loads on individual systems that do not occur in the same time interval. Meaningful only when considering losds within a limited period of time, such as a day, week, month, a heating or cooling season, and usually for not more than I year.

North American Eleatric Rejiability Counci (NERC): A council formed in 1968 by the electric utility industry to promote the reliability and ade. quacy of bulk power supply in the electric utility systems of North America. NERC cansists of ten regional reliability councils and encompasses essentially all the power regions of the contiguous United States, Canada, and Mexico. The NERC Regions are:

ASCC - Alaskan System Coordination Council

ECAR - East Ceniral Area Reliability

Cootdination Agrement

ERCOT - Electric Reliability Council of Texas

MAIN - Mid-America Interconnected Network

MAAC - Mid-Atlantic Area Council

MAPP - Mid-Continenl Area Power Pool

NPCC - Northeast Power Coordinating Council 
SERC - Southeastem Electric Reliability Council

SPP - Southwest Power Pool

WSCC - Western Systems Coordinating Council

Other Costs: A residual category to capture the Indirect Costs of DSM programs that cannot be meaningfolly included in any of the other cost calegories listed and defined herejn. Included are costs such as those incurred in the research and development of DSM technologies.

Other DSM Programs: A residual category to capture the effects of DSM programs that cannot be meanisgfully included in any of the program categories listed and defined berein. The energy effects attributable to this category should be the net effects of all the residual programs. Programs that promote consumer's substitution of electricity by other energy types shonld be included in Other DSM Programs. Atso, self-genetation should be inciuded in Other DSM Programs to the extent that it is not accounted for as backup generation in Other Load Management or Interruptible Load categories.

Other Incentives: Energy Efficiency programs that offer cash or noncash awards to electric energy effjciency deliverers, such as appliance and equipment dealers, building contractors, and architectural and engineering firms, that encourage consumer particjpation in a DSM program and adoption of recommended measures.

Other Load Manogenent: Refers to programs other than Direct Load Control and Interruptible Load that limit or shift peak load from on-peak to off-peak timte periods. It includes technologies that primarily shift all or part of a load from one time-of-day to another and secondarily may have an impact on energy consumption. Examples include space heating and water healing starage systems, cool storage systems, and load limiting devices in energy managemeat systems. This category aiso includes programs that aggressively promote time-of-use (TOU) rates and other jnoovative rates such as real time poicing. These rates are intended to reduce constumer bills and shift hours of operation of equipment from on-peak to off-peak periods through the application of time-differentiated rates.

Ontage: The perjod during which a generating unit, transmission libe, or other facility is out of service.

Peal Demand: The maximum load during a specified period of time.

Penking Capacity: Capacity of generating equipment normally reserved for operation during the hours of highest daily, weekly, or seasonal loads. Some generatiog equipment may be operated at certain times as peaking capacity and at other times to sorve loads on an aronnd-the-clock basis.

Percent Difference: The relative change in a quanlity over a specified time period. It is calculated as follows: the current value has the previous valne subtracted from it; this new number is divided by the absolute value of the previous valute; then this now number is multiplied by $\mathbf{1 0 0}$.

Plinned Generator: A proposal by a company to install electric generating equipment at an existing or planned facility ot site. The proposal is based on the owner having obtained (1) all environmental and regulatory approvals. (2) a signed contract for the electric energy, or (3) financial closure for the facility.

Potential Penk Logd Redection: The amount of annual peak load reduction capability (measured in kilowatts) that can be deployed from Dlrect Load Control, Interruptible Load, Other Load Management. and Other DSM Program activities. It represents the load that can be reduced either by the direct control of the utility system operator or by the consumer in response to a utility reguest to curtail toad. It teflects the installed load reduction capability, as opposed to the Actual Peak Reduction achieved by participants. doring the time of anbual system peak load.

Power: The rate at which energy is transferred. Electrical energy is usually measured in watts. Also used for a messurement of capseity.

Power Pool: An association of two or more interconnected electric systems having an agreement to coordinate operations and planning for improved reliability and efficiencies.

Process Heating: Energy Efficiency program promotion of increased electric energy efficiency applications in industrial process heating.

Pablic Street and Highway Lighting: Public street and highway lighting includes tectricity supplied and services rendered for the purposes of lighting streets, bighways, parks, and other public places; or for traffic or olher signal system service, for municipalities, or other divisions or agencies of State or Federal governments.

Rate Bast: The value of property upon which a ulility is permitted to earn a specified rate of returd as established by a regulatory authority. The rate base geperally represents the value of property used by the utility in providing service and may be calculated by any one or a combination of the following accounting metbods: fair value, prudent investment, reproxduction cost, or original cost. Dependiog on which method is used, the rate base includes cash, working capital, materials and supplies, and deductions for accumulated provisions for depreciation, contributions in aid of construction, customer advances for construction, accumulated deferted income taxes, and accumelated deferred investment tax credits.

Ratemakiog Anthority: A utility commission's legal authority to fix, modify, approve, or disapprove rates, as determined by the powers given the commission by a State or Federal legislature.

Rezulation: The govenmental function of controlling or directing economic entities through the process of rolemaking and adjudication. 
keserve Margin (Opernting): The anount of unused available capability of an electric power system at peak load for a utility systern as a per* centage of total capability.

Residential: The residential sector is defined as private household establishments whith consume energy primarily for space heating, water heating, air conditioning. lighting. refrigeration, cooking and clothes drying. The classification of an individual consumer's account, where the use is both residential and commercial, is based on priscipal use.

Retailt: Sales covering electrical energy supplied for residential, commercial, and industrial end-use purposes. Other small classes, such as agriculture and street lighting, also are included in this calegory.

Revenue: The total amount of money received by a firm from sales of its products and/or services, gajns from the sales or exchange of assets, interest and divjdends earned on investments, and other increases in the owner's oquity except those arising from capital adjustments.

Sales: The amount of kilowatthonrs sold in a given period of time; usualty grouped by classes of service, such as residential, commercigl, industria!., and other. Other sales include public street and bighway lighting, other sales to public authorities and railways, and interdepartmental sales.

Sales for Resale: Energy supplied to other electric utilities, cooperatives, municipalities, and Federal and State electric agencies for resalt to ultimale consumers.

Standard Industrial Clessiffeation (SICr: A set of codes developed by the Office of Management and Budget, which categorizes business into groups with similar economic activities.

System (Electric): Physically connected genteration, transmission, and distribution facilities operated as an integrated unit ander one central management, or operating supervision.

Tatal DSM Cost: Refers to the sum of total utility cosi and nonutility cost.

Total DSM Programs: Refers to the total net effects of all the utility's DSM programs. For the purpose of this survey, it is the sum of the effeets for Energy Efficiency, Direct Load Control, Interruptible Load, Other Load Management, Other DSM Programs, and Load Building. Net growth in enersy or load effects should be reported as a negative number, shown with a diknus sign.

Total Nonutility Costs: Refers to total cash expendjtures incurred by consumers and trade allies that are associated with participation in a DSM program, but that are not reimbursed by the gtility. The nonutility expenditures should include only those atditional costs necessary to purchise or install tn efficient measure relative to a less efficient one. Costs are to be reported in nominal Gollars is the year in which they are incurred, regardless of when the actual effects occur. To the extent possible, respondents are asked to provide the best estimate of nonutility costs if actial costs are unavailable.

Total Utility Costs: Refers to the sum of the totol Direct and Indirect Utility Costs for the year. Utility costs should reflect the total cash expenditures for the year, reported in nominal dollars, that flowed out to support DSM programs. They should be reported in the year they are incurred, regardless of when the actual effects ocevr.

Transmisston: The movement or transfer of electric energy over an interconnected groop of limes and associated equipmeat between points of supply and points at which it is transformed for delivery to consumers, or is delivered to other electric systems. Transmission is considered to end when the energy is transforted for distribution to the consumer.

Transmbstion System (Electric): An intercounected group of electric transmission lines and associated equipment for moving or transferring electric energy in bulk between points of supply and points at which it is transformed for delivery over the distribution system lines to consuners, or is delivered to other electric systems.

Uniform System of Aceonnts: Prescribed funancial nules and regulations established by the Federal Energy Regulatory Conmission for uilities subject to its jurisdiction under the authority granted by the Federal Power Act.

Utility-Earned Incentives: Costs in the form of incentives paid to the utility for achievement in consumer particjpation in DSM programs. These financial inceptives are intended to inflaence the ntility's consideration of DSM as a resource option by addressing cost recovery, lost revenue, and profitability.

Voltage Reduction: Any intentional reduction of system voliage by 3 percent or greater for reasons of maintaining the continuity of service of the bulk electric power supply system.

Water Heating: Energy Efficiency program promotion to increase efficiency in water beating. including low-flow shower heads and water beater insulation wraps. Could be applicable to residential, commercial, or industrial eonsumer sectors.

Wutt: The electrical unit of power. The rate of energy transfer equivalent to 1 ampere flowing under a pressure of 1 volt at unity power factor.

Watthour (Wh): An electrical energy unit of measure equat to 1 watt of power supplied to, er taten from, an electric circuit steadily for 1 hour.

Wheeling Service: The movement of electricity from one system to another over transmission facilities of intervening systems. Wheeling service contracts can be established between two or more systems. 
Wholesske Sales: Energy supplied to other electric utilities, cooperatives, municipals, and Federal and
State electric agencies for resale to ultimate consumers. 
Enerzy Information Administration Consumption Surveys:

The Energy Information Administration (EIA) also conducts consumption surveys that provide detailed information on how different consumers use exergy. In recent surveys, DSM data bas been collected as part of the data collection for three EIA consumption surveys: the Residential Energy Consumption Survey, the Commertia] Buildings Energy Consumption Survey, and the Manufacturing Energy Consumption Survey. The following provides a brief description of each of these surveys.

Residential Energy Consumption Survey (RECS): Since 1978, EIA has collected data froci U.S. housebolds about how they use energy and billing data from their energy suppliess sbout how much energy they use. In the ninth RECS underlaken in 1993, over 7,000 households were surveyed and the results are extrapolated to 97 million households. The triennial survey collects data on housing characteristtcs, energy consumption and expenditutes, stock of energy-using appliances, and energy-related behavior.

Questions about household participation in DSM prograus were arked in the 1990 and 1993 RECS. Data can be found in Housing Characteristics 1990 (DOENEIA-0314(90)), Household Energy Consumption and Expenditures 1990 (DOE/EIA-0321(9))), and Housing Characteristics 1993 (19bles available in November 1994 and report available in spring 1995). The data show participation by type of DSM program in botb surveys. Additionally. the 1993 survey shows household perceptions of the availability of DSM programs.

Por further information concersing the RECS DSM data or the RECS in general, please contact Robern Latta, RECS Manager, at (202) 586-1385, FAX at (202) 586-0018, or Internot E-mail rlatio eia.doe.gov.

Manufactnring Enerby Consumption Sarvey (MECS): The MECS was first conducted for 1985 and presents data representing all but the smallest ntanufacturing establishments. It is a triennial survey that collects data on energy consumption and related issues in manufacturing establishments. The 1991 MECS presents separate estimates for all 20 major industrial gToups from the manufacturing sector as defined by the Standard Industrial Classificalion (SIC) Codes. Within these major groups, separate estimates are presented for $\mathbf{4 2}$ industries and industry groups.

New to the 1991 version of the MBCS are datg on energy efficiency activities and DSM in particular. The data tables are available now in electronic form on EPUBS and in a forthcouing publicalion. The tables present participation by SIC Code, type of progran, and whether electric utilities are involved. Due to the sample design, data must be presented in terms of ener;jy consumption rather than cotunts of establishments. In future years, both types of measures are expected to be avilable.

For further information concerning DS]M data or any aspect of the MECS, please contact Mark Shipper, MECS Survey Managet, at (202) 586-1136, FAX at (202) 586-0018, or Internet E-mail mshipper Geia.doe.gov,

Comprercial Buildiags Emergy Consumption Surrey (CBECS):Since 1979, EIA has collected data on the physical and operating characteristics that affect energy use in U.S. commercial buildings. Billing data containing energy consumption and expenditures are collected from the energy suppliers to these brildings. In the fifth CBECS undertaken in 1992, both the building respondents and the energy suppliers were asked extensive questions about the types of DSM progtams that the buildings participated in, the sponsors of those programs. and the types of assistance that was provided through the DSM programs. DSM participation data as reported by the bujlding owners, managers, and tenants can be found in Commercial Buildings Characteristics 1992 (DOEJEIA-00246(92)).

For further information concerning the CBECS DSM data or the CBECS in general, please contact Martha Johnson, CBECS Manager, at (202) 586-1135, FAX at (202) 586-0018, or Internet E-majl mjohnson \& eis.doe.gov, 